Supporting Information

\title{
Transition Metal Doped Aluminum Clusters: An Account of Spin Turbasu Sengupta, ${ }^{\dagger}$ Susanta Das, ${ }^{,+\dagger}$ and Sourav Pal ${ }^{*,+}$
}

\author{
${ }^{\dagger}$ Physical Chemistry Division, CSIR National Chemical Laboratory, Pune 411008, India, \\ ${ }^{\ddagger}$ Department of Chemistry, Indian Institute of Technology Bombay, Powai, Mumbai 400076, India \\ E-mail: susanta.das@biu.ac.il; spal@chem.iitb.ac.in
}

*To whom correspondence should be addressed 


\section{Content Index}

Title

Page Number

1. Table of energies of optimized impure clusters in different spin states

S3

2. Table of calculated Fukui functions of pristine clusters

3. Thermodynamic data of $\mathrm{CO}_{2}$ adsorption on impure aluminum clusters (linear and Parallel)

4. Cartesian coordinates and normal modes of vibrations of pristine clusters

S22-S23

5. Cartesian coordinates and normal modes of vibrations of $\mathrm{Al}_{5} \mathrm{M}$ clusters and $\mathrm{Al}_{5} \mathrm{M}-\mathrm{CO}_{2}$ complexes

S24-S48

6. Cartesian coordinates and normal modes of vibrations of $\mathrm{Al}_{7} \mathrm{M}(\mathrm{a})$ clusters and $\mathrm{Al}_{5} \mathrm{M}(\mathrm{a})-\mathrm{CO}_{2}$ complexes

S49-S86

7. Cartesian coordinates and normal modes of vibrations of $\mathrm{Al}_{7} \mathrm{M}(\mathrm{b})$ clusters and $\mathrm{Al}_{5} \mathrm{M}(\mathrm{b})-\mathrm{CO}_{2}$ complexes

S87-S111

8. Cartesian coordinates and normal modes of vibrations of $\mathrm{Al}_{7} \mathrm{M}(\mathrm{c})$ clusters and $\mathrm{Al}_{5} \mathrm{M}(\mathrm{c})-\mathrm{CO}_{2}$ complexes

S112-S149

9. Additional information

S150-S152

$\mathrm{S} 153$

10.Thermodynamic Data Plot of $\mathrm{CO}_{2}$ Adsorption on Doped aluminum clusters in PBE-PBE functional

S154-S156 11. Thermochemical Da
PBE-PBE functional.

12.Cartesian Coordinates and Normal Modes of Vibrations of $\mathrm{Al}_{5} \mathrm{M}$ and $\mathrm{Al}_{7} \mathrm{M}(\mathrm{c})$ clusters and $\mathrm{CO}_{2}$ complexes in dispersion corrected PBE-PBE functional

S157-S175

13. Comparison of standard DFT and DFT-D results for PBE-PBE functional 
Energies of optimized impure clusters in different spin states [M06-2X/6-311G (d,p)]

(minimum energies are highlighted in 'red', clusters with minimum spin ground state are highlighted in 'Green')

(Lowest energies obtained in each spin state are shown)

\begin{tabular}{|c|c|c|c|c|c|c|c|c|c|c|c|c|c|c|c|}
\hline \multicolumn{16}{|c|}{$\begin{array}{c}\mathrm{Al}_{5} \mathbf{M} \text { Clusters } \\
\text { Table S1 }\end{array}$} \\
\hline $2 \mathrm{~S}+1$ & 1 & 2 & 3 & 4 & 5 & 6 & 7 & 8 & 9 & 10 & 11 & 12 & 13 & 14 & 15 \\
\hline Sc & -1258.379345 & & -1258.386728 & & -1258.379599 & & -1258.325667 & & -1258.300795 & & -1258.25607 & & -1258.101133 & & -1257.949063 \\
\hline Ti & & -1269.974076 & & -1269.992237 & & -1269.98391 & & -1269.976398 & & -1269.940949 & & -1269.852476 & & \begin{tabular}{|l|}
-1269.7147 \\
\end{tabular} & \\
\hline $\bar{V}$ & -1283.182988 & & -1283.256407 & & -1283.266212 & & -1283.256732 & & -1283.237998 & & -1283.215861 & & -1283.144804 & & -1283.010396 \\
\hline $\mathrm{Cr}$ & & -1298.288704 & & -1298.298215 & & -1298.310167 & & -1298.297679 & & -1298.281209 & & -1298.266553 & & -1298.190079 & \\
\hline Mn & -1315.782551 & & -1315.846785 & & -1315.908944 & & \begin{tabular}{|l|}
-1315.92768 \\
\end{tabular} & & -1315.909844 & & -1315.885686 & & -1315.853588 & & -1315.763198 \\
\hline $\mathrm{Fe}$ & & -1335.422049 & & -1335.430574 & & -1335.419301 & & -1335.417427 & & -1335.374531 & & -1335.343385 & & -1335.251261 & \\
\hline Co & -1357.0227 & & -1357.095494 & & -1357.082059 & & -1357.066849 & & -1357.015713 & & -1356.986284 & & -1356.882875 & & -1356.737168 \\
\hline$\overline{\mathrm{Ni}}$ & & -1381.355267 & & -1381.343174 & & -1381.328481 & & -1381.314301 & & -1381.24605 & & -1381.120529 & & \begin{tabular}{|l|}
-1380.96219 \\
\end{tabular} & \\
\hline$\overline{\mathrm{Cu}}$ & -1408.24351 & & -1408.229636 & & \begin{tabular}{|l|l|}
-1408.219282 \\
\end{tabular} & & -1408.168986 & & -1408.137186 & & -1408.002791 & & -1407.833489 & & -1407.634686 \\
\hline $\mathrm{Zn}$ & & -1277.460165 & & -1277.450996 & & -1277.443127 & & -1277.378512 & & -1277.311746 & & -1277.146724 & & -1276.981969 & \\
\hline
\end{tabular}

\section{$\mathrm{Al}_{7} \mathrm{M}$ (a) Clusters}

Table S2

\begin{tabular}{|c|c|c|c|c|c|c|c|c|c|c|c|c|c|c|c|}
\hline $2 \mathrm{~S}+1$ & 1 & 2 & 3 & 4 & 5 & 6 & 7 & 8 & 9 & 10 & 11 & 12 & 13 & 14 & 15 \\
\hline ic & -1743.282723 & & -1743.29357 & & \begin{tabular}{|l}
-1743.289247 \\
\end{tabular} & & -1743.24238 & & \begin{tabular}{|l|}
-1743.180865 \\
\end{tabular} & & \begin{tabular}{|l|}
-1743.147081 \\
\end{tabular} & & -1743.053266 & & -1742.938051 \\
\hline$\overline{T i}$ & & -1754.895083 & & $\begin{array}{l}-1754.90368 \\
\end{array}$ & & -1754.897204 & & -1754.853048 & & -1754.824623 & & -1754.774181 & & -1754.658202 & \\
\hline & -1768.091545 & & -1768.168241 & & -1768.173672 & & -1768.167361 & & -1768.124104 & & -1768.072246 & & -1768.02909 & & -1767.931691 \\
\hline $\mathrm{Cr}$ & & -1783.117346 & & -1783.216102 & & -1783.219501 & & -1783.214056 & & -1783.169939 & & -1783.114269 & & -1783.073458 & \\
\hline$\overline{M n}$ & -1800.682804 & & \begin{tabular}{|l|}
-1800.83276 \\
\end{tabular} & & -1800.836752 & & -1800.83354 & & -1800.821439 & & -1800.786131 & & -1800.718503 & & -1800.682387 \\
\hline $\mathrm{Fe}$ & & -1820.296428 & & -1820.341769 & & -1820.337408 & & -1820.310733 & & -1820.265731 & & -1820.238255 & & -1820.168344 & \\
\hline$\overline{C 0}$ & -1841.925339 & & -1842.002462 & & -1841.977317 & & -1841.958376 & & -1841.925862 & & -1841.864544 & & -1841.781282 & & -1841.694669 \\
\hline$\overline{\mathrm{Ni}}$ & & -1866.261111 & & -1866.257701 & & -1866.219312 & & -1866.186113 & & -1866.124304 & & -1866.029473 & & -1865.939749 & \\
\hline $\mathrm{Cu}$ & -1893.152316 & & -1893.149162 & & -1893.108714 & & -1893.06069 & & -1893.013457 & & -1892.919294 & & -1892.830168 & & -1892.68719 \\
\hline $\mathbf{Z n}$ & & -1762.357087 & & -1762.342911 & & -1762.309668 & & -1762.25625 & & -1762.210525 & & -1762.116597 & & -1762.005072 & \\
\hline
\end{tabular}

\section{$\mathrm{Al}_{7} \mathbf{M}(\mathbf{b})$ Clusters}

Table S3

\begin{tabular}{|c|c|c|c|c|c|c|c|c|c|c|c|c|c|c|c|}
\hline $2 \mathrm{~S}+1$ & 1 & 2 & 3 & 4 & 5 & 6 & 7 & 8 & 9 & 10 & 11 & 12 & 13 & 14 & 15 \\
\hline ic & 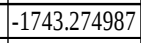 & & -1743.294497 & & -1743.289664 & & -1743.238186 & & -1743.202713 & & -1743.16672 & & -1743.060856 & & -1742.952527 \\
\hline & & -1754.89516 & & -1754.903645 & & -1754.893612 & & -1754.847892 & & -1754.811997 & & -1754.774131 & & -1754.666161 & \\
\hline & -1768.091245 & & -1768.168487 & & -1768.173594 & & -1768.167346 & & \begin{tabular}{|l|l|}
-1768.12412 \\
\end{tabular} & & -1768.073701 & & 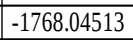 & & -1767.943813 \\
\hline r & & -1783.117365 & & -1783.216064 & & -1783.219461 & & -1783.214007 & & -1783.169984 & & -1783.119214 & & -1783.090341 & \\
\hline Mn & -1800.682743 & & -1800.832801 & & -1800.836772 & & -1800.833673 & & $-1800.82160 \leq$ & & -1800.778058 & & $\begin{array}{l}-1800.75166 \\
\end{array}$ & & -1800.676276 \\
\hline se & & -1820.296071 & & -1820.341767 & & -1820.337388 & & -1820.31085 & & -1820.261245 & & -1820.219067 & & -1820.162121 & \\
\hline 0 & -1841.925439 & & -1842.002932 & & -1841.99811 & & -1841.959491 & & -1841.908297 & & -1841.877582 & & -1841.781282 & & -1841.694669 \\
\hline ii & & -1866.26126 & & -1866.257722 & & -1866.188664 & & -1866.186 & & -1866.140785 & & \begin{tabular}{|l|l|}
-1866.036981 \\
\end{tabular} & & -1865.936671 & \\
\hline Cu & -1893.152312 & & -1893.149157 & & -1893.108705 & & -1893.076695 & & $-1893.02608^{5}$ & & -1892.928155 & & -1892.824153 & & -1892.710021 \\
\hline In & & -1762.353417 & & -1762.346345 & & -1762.304262 & & -1762.2653 & & $|-1762.203461|$ & & -1762.120276 & & -1762.004106 & \\
\hline \multicolumn{16}{|c|}{$\begin{array}{c}\mathrm{Al}_{7} \mathbf{M}(\mathrm{c}) \text { Clusters } \\
\text { Table S4 }\end{array}$} \\
\hline $2 \mathrm{~S}+1$ & 1 & 2 & 3 & 4 & 5 & 6 & 7 & \begin{tabular}{|l|} 
\\
8
\end{tabular} & 9 & 10 & 11 & 12 & 13 & 14 & 15 \\
\hline & -1743.278008 & & -1743.294026 & & 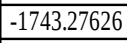 & & -1743.245066 & & -1743.207433 & & -1743.155976 & & -1743.066252 & & -1742.961358 \\
\hline$[i$ & & -1754.895348 & & -1754.902896 & & -1754.897263 & & -1754.853221 & & \begin{tabular}{|l|}
-1754.824524 \\
\end{tabular} & & -1754.783055 & & \begin{tabular}{|l|}
-1754.694194 \\
\end{tabular} & \\
\hline & -1768.091246 & & -1768.168495 & & -1768.173594 & & -1768.167349 & & 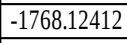 & & -1768.073702 & & -1768.045133 & & -1767.943811 \\
\hline Cr & & -1783.152779 & & -1783.182969 & & -1783.204125 & & -1783.190649 & & \begin{tabular}{|l|}
-1783.14413 \\
\end{tabular} & & 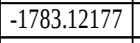 & & \begin{tabular}{|l|}
-1783.062288 \\
\end{tabular} & \\
\hline In & -1800.659976 & & -1800.753795 & & -1800.824649 & & -1800.815169 & & -1800.80889 & & -1800.777358 & & -1800.746857 & & -1800.682276 \\
\hline se & & -1820.248346 & & \begin{tabular}{|l|l|l}
-1820.34396 \\
\end{tabular} & & -1820.296155 & & -1820.275561 & & \begin{tabular}{|l|}
-1820.251794 \\
\end{tabular} & & \begin{tabular}{|l|}
-1820.220333 \\
\end{tabular} & & \begin{tabular}{|l|}
-1820.16086 \\
\end{tabular} & \\
\hline 50 & -1841.925437 & & -1842.002932 & & -1841.998111 & & -1841.959491 & & -1841.908296 & & -1841.880517 & & -1841.781282 & & -1841.696318 \\
\hline ii & & 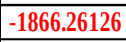 & & -1866.257725 & & -1866.188664 & & -1866.186006 & & \begin{tabular}{|l|}
-1866.14078 \\
\end{tabular} & & \begin{tabular}{|l|}
-1866.03702 \\
\end{tabular} & & \begin{tabular}{|l|}
-1865.936674 \\
\end{tabular} & \\
\hline cu & -1893.152274 & & -1893.14915 & & -1893.102715 & & -1893.071537 & & -1893.03026 & & -1892.948204 & & -1892.850493 & & -1892.730025 \\
\hline In & & -1762.353418 & & -1762.346345 & & 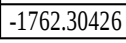 & & -1762.265296 & & \begin{tabular}{|l|}
-1762.203461 \\
\end{tabular} & & \begin{tabular}{|l|}
-1762.120276 \\
\end{tabular} & & \begin{tabular}{|l|}
-1762.004107 \\
\end{tabular} & \\
\hline
\end{tabular}




\section{Calculated Fukui functions of pristine clusters [M06-2X/6-311G (d,p)]}

\section{(Hirshfeld population analysis is used)}

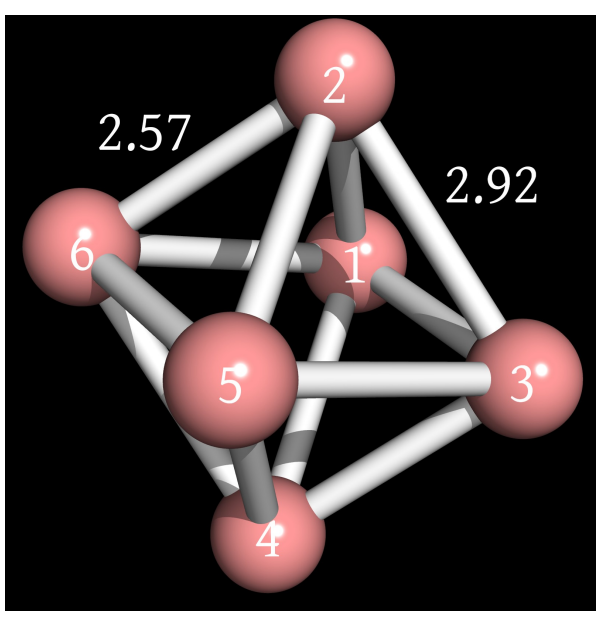

Figure S1

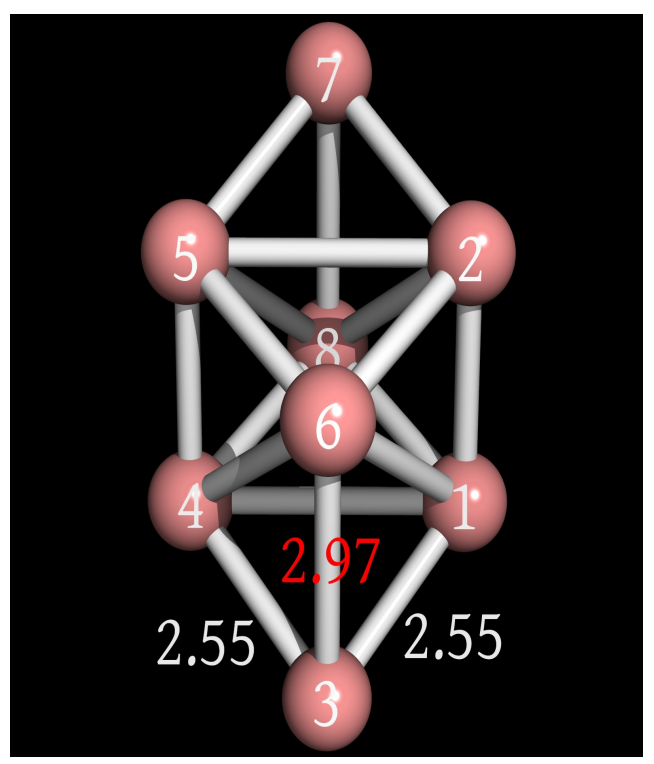

Figure S2

\section{Table S5:AI 6 cluster}

\begin{tabular}{|c|c|c|c|}
\hline Al & $\boldsymbol{f}^{+}$ & Al & $\boldsymbol{f}^{-}$ \\
\hline 1 & 0.166829 & 1 & 0.166671 \\
\hline 2 & 0.166791 & 2 & 0.166725 \\
\hline 3 & 0.166812 & 3 & 0.166676 \\
\hline 4 & 0.166791 & 4 & 0.166725 \\
\hline 5 & 0.166829 & 5 & 0.166671 \\
\hline 6 & 0.166812 & 6 & 0.166676 \\
\hline
\end{tabular}

All Sites are equivalent considering three digits after decimal

\section{Table S6:Al ${ }_{8}$ cluster}

\begin{tabular}{|c|c|c|c|}
\hline $\mathrm{Al}$ & $\boldsymbol{f}^{+}$ & $\mathrm{Al}$ & $\boldsymbol{f}^{-}$ \\
\hline 1 & 0.131623 & 1 & 0.121059 \\
\hline 2 & 0.131623 & 2 & 0.121062 \\
\hline 3 & 0.153563 & 3 & 0.186574 \\
\hline 4 & 0.131624 & 4 & 0.121059 \\
\hline 5 & 0.131625 & 5 & 0.121062 \\
\hline 6 & 0.083603 & 6 & 0.071409 \\
\hline 7 & 0.153563 & 7 & 0.186569 \\
\hline 8 & 0.083603 & 8 & 0.071408 \\
\hline
\end{tabular}

Three types of chemically distinct sites (highlighted with different colors) 
Thermochemical Data of $\mathrm{CO}_{2}$ adsorption on impure aluminum clusters

\section{Two types of binding mode}

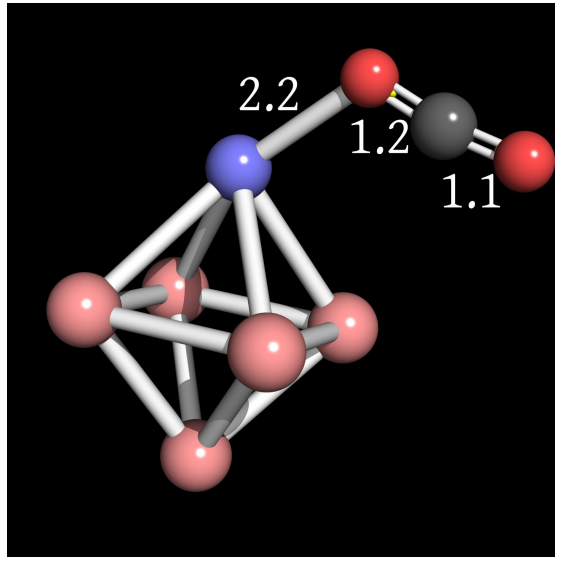

Figure S3

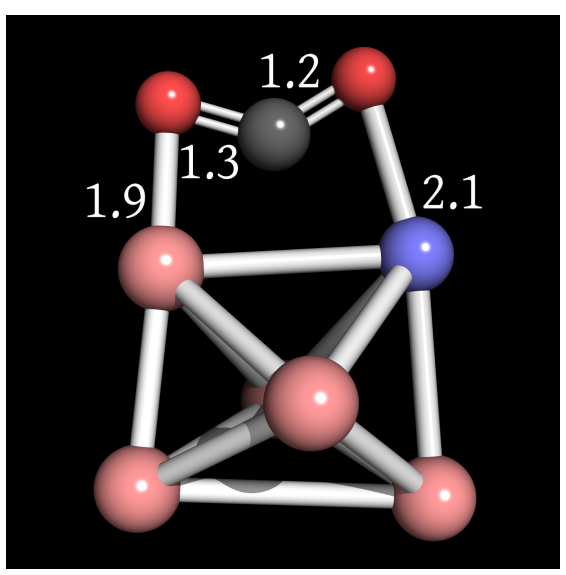

Figure S5

$\mathrm{Al}_{5} \mathrm{M}---\mathrm{CO}_{2}$
Linear Binding

(weak)

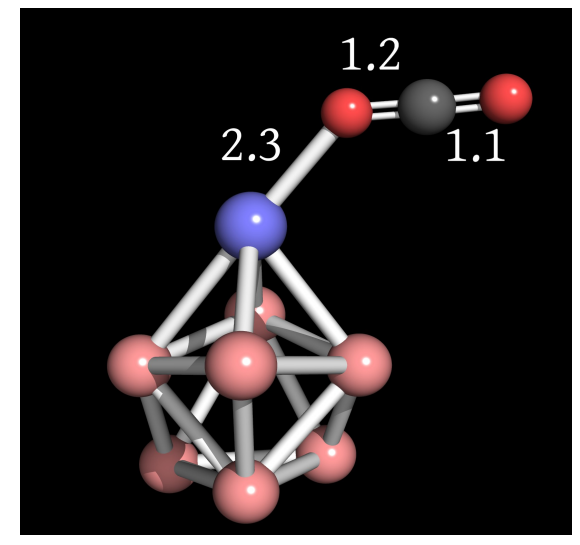

Figure S4

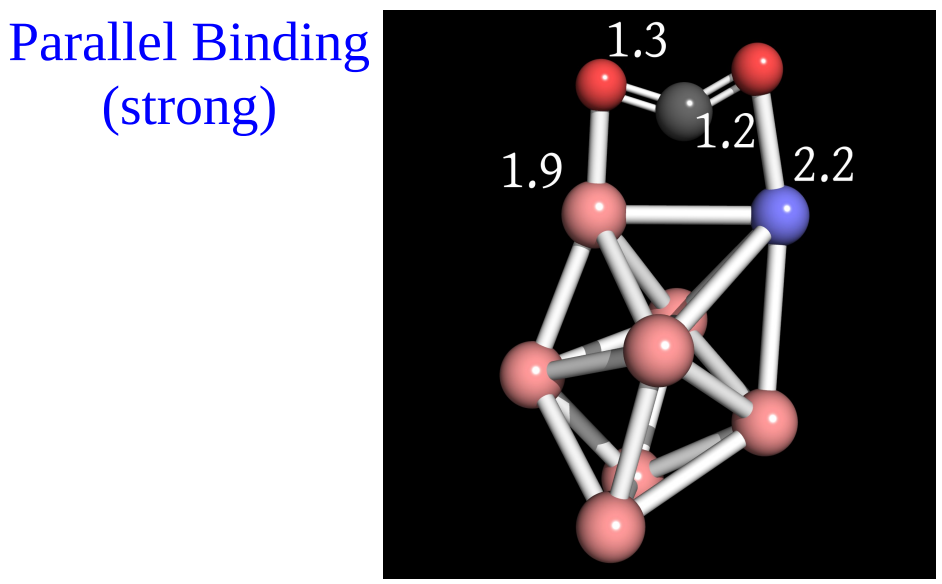

Figure S6

Sample Figures of two types of binding mode of $\mathrm{CO}_{2}$ with impure aluminum clusters 
Thermochemical Data of $\mathrm{CO}_{2}$ adsorption on $\mathrm{Al}_{5} \underline{\mathrm{M} \text { clusters }}$

(i)Table S7: Linear Binding Mode(M06-2X)

\begin{tabular}{|c|c|c|c|c|c|c|}
\hline Dopant & Parameters & Cluster+CO2 & doped cluster & CO2 & Delta(au) & in kcal/mol \\
\hline \multirow[t]{2}{*}{ Sc } & $\mathrm{U}$ & -1446.970223 & -1258.386728 & -188.558113 & -0.025382 & -15.92745882 \\
\hline & U+therm & -1446.956957 & -1258.377257 & -188.555511 & -0.024189 & -15.17883939 \\
\hline \multirow[t]{2}{*}{3} & $\mathrm{H}$ & -1446.956012 & -1258.376313 & -188.554567 & -0.025132 & -15.7705813201 \\
\hline & $\bar{G}$ & -1447.013937 & -1258.423839 & -188.578788 & -0.01131 & -7.0971380999 \\
\hline \multirow[t]{2}{*}{$\mathrm{Ti}$} & $\overline{\mathrm{U}}$ & -1458.567022 & -1269.983912 & -188.558113 & -0.024997 & -15.68586747 \\
\hline & U+therm & -1458.55407 & -1269.974126 & -188.555511 & -0.024433 & -15.3319518299 \\
\hline \multirow[t]{2}{*}{4} & $\mathrm{H}$ & -1458.553126 & -1269.973182 & -188.554567 & -0.025377 & -15.9243212701 \\
\hline & $\bar{G}$ & -1458.611731 & -1270.022287 & -188.578788 & -0.010656 & -6.68674656 \\
\hline & & & & & & \\
\hline \multirow[t]{2}{*}{$\mathrm{V}$} & $\overline{\mathrm{U}}$ & -1471.8461 & -1283.266212 & -188.558113 & -0.021775 & -13.66403025 \\
\hline & U+therm & -1471.832424 & -1283.256705 & -188.555511 & -0.020208 & -12.6807220799 \\
\hline \multirow[t]{2}{*}{5} & $\mathrm{H}$ & -1471.831479 & -1283.255761 & -188.554567 & -0.021151 & -13.2724640101 \\
\hline & $\bar{G}$ & -1471.892643 & -1283.304383 & -188.578788 & -0.009472 & -5.94377472 \\
\hline \multirow[t]{2}{*}{$\mathrm{Cr}$} & $\overline{\mathrm{U}}$ & -1486.884824 & -1298.310167 & -188.558113 & -0.016544 & -10.3815254399 \\
\hline & U+therm & -1486.871101 & -1298.300584 & -188.555511 & -0.015006 & -9.4164150599 \\
\hline \multirow[t]{2}{*}{6} & $\mathrm{H}$ & -1486.870157 & -1298.29964 & -188.554567 & -0.01595 & -10.0087845001 \\
\hline & $\bar{G}$ & -1486.93169 & -1298.348616 & -188.578788 & -0.004286 & -2.6895078599 \\
\hline \multirow[t]{2}{*}{$\mathrm{Mn}$} & $\overline{\mathrm{U}}$ & -1504.491176 & -1315.909844 & -188.558113 & -0.023219 & -14.57015469 \\
\hline & U+therm & -1504.476801 & -1315.899869 & -188.555511 & -0.021421 & -13.4418917099 \\
\hline \multirow[t]{2}{*}{7} & $\mathrm{H}$ & -1504.475857 & -1315.898924 & -188.554567 & -0.022366 & -14.0348886599 \\
\hline & $\bar{G}$ & -1504.539878 & -1315.949255 & -188.578788 & -0.011835 & -7.42658085 \\
\hline & & & & & & \\
\hline \multirow[t]{2}{*}{$\mathrm{Fe}$} & $\mathrm{U}$ & -1523.952239 & -1335.430369 & -188.558113 & 0.036243 & 22.74284493 \\
\hline & U+therm & -1523.93853 & -1335.42043 & -188.555511 & 0.037411 & 23.47577661 \\
\hline \multirow[t]{2}{*}{4} & $\mathrm{H}$ & -1523.937586 & -1335.419486 & -188.554567 & 0.036467 & 22.8834071699 \\
\hline & $\mathrm{G}$ & -1523.997547 & -1335.469746 & -188.578788 & 0.050987 & 31.99485237 \\
\hline \multirow[t]{2}{*}{$\mathrm{Co}$} & $\mathrm{U}$ & -1545.651937 & -1357.082057 & -188.558113 & -0.011767 & -7.38391017 \\
\hline & U+therm & -1545.638033 & -1357.07221 & -188.555511 & -0.010312 & -6.4708831199 \\
\hline \multirow[t]{2}{*}{3} & $\mathrm{H}$ & -1545.637088 & -1357.071265 & -188.554567 & -0.011256 & -7.06325256 \\
\hline & $\bar{G}$ & -1545.69864 & -1357.120854 & -188.578788 & 0.001002 & 0.62876502 \\
\hline \multirow{2}{*}{$\mathrm{Ni}$} & $\bar{U}$ & -1569927911 & -1381355267 & -188558113 & 0014521 & 9 11834781 \\
\hline & U+therm & |1569.914388 & -1381.345789 & $\frac{-100.50011}{-188.555511}$ & $\frac{-0.014501}{-0.013088}$ & $\begin{array}{l}-8.11054 / 01 \\
-8.21285088\end{array}$ \\
\hline \multirow[t]{2}{*}{2} & $\mathrm{H}$ & -1569.913444 & -1381.344844 & -188.554567 & -0.014033 & -8.8058478301 \\
\hline & $\bar{G}$ & -1569.972921 & -1381.392373 & -188.578788 & -0.00176 & -1.1044176001 \\
\hline \multirow{2}{*}{$\mathrm{Cu}$} & $\overline{\mathrm{U}}$ & -1596.805184 & -1408.238474 & -188.558113 & -0.008597 & -5.39470347 \\
\hline & U+therm & -1596.790809 & -1408.228475 & -188.555511 & -0.006823 & -4.2815007301 \\
\hline \multirow[t]{2}{*}{1} & $\mathrm{H}$ & -1596.789865 & -1408.227531 & -188.554567 & -0.007767 & -4.87387017 \\
\hline & $\bar{G}$ & -1596.853231 & -1408.276748 & -188.578788 & 0.002305 & 1.44641055 \\
\hline & & & & & & \\
\hline \multirow[t]{2}{*}{$\mathrm{Zn}$} & $\mathrm{U}$ & -1466.025232 & -1277.460163 & -188.558113 & -0.006956 & -4.36495956 \\
\hline & $\overline{\mathrm{U}+\text { therm }}$ & -1466.010661 & -1277.449931 & -188.555511 & -0.005219 & -3.2749746899 \\
\hline \multirow[t]{2}{*}{2} & $\mathrm{H}$ & -1466.009717 & -1277.448987 & -188.554567 & -0.006163 & -3.86734413 \\
\hline & $\mathrm{G}$ & -1466.07338 & -1277.49991 & -188.578788 & 0.005318 & 3.33709818 \\
\hline
\end{tabular}


(ii)Table S8: Linear Binding Mode(PBE-PBE)

\begin{tabular}{|c|c|c|c|c|c|c|}
\hline Dopant & Parameters & Cluster+CO2 & doped cluster & $\mathrm{CO} 2$ & Delta(in a.u.) & in $\mathrm{kcal} / \mathrm{mol}$ \\
\hline Sc & $\mathrm{U}$ & & -1257.916165 & -188.43982 & & \\
\hline \multirow[t]{3}{*}{3} & $\mathrm{U}+$ therm & & -1257.906823 & -188.437166 & & \\
\hline & $\mathrm{H}$ & no binding & -1257.905879 & -188.436222 & & \\
\hline & $\mathrm{G}$ & found & -1257.952818 & -188.460545 & & \\
\hline $\mathrm{Ti}$ & $\bar{U}$ & & & & & \\
\hline \multirow[t]{3}{*}{4} & U+therm & -1457.962941 & -1269.510298 & -188.437166 & -0.015477 & -9.7119722701 \\
\hline & $\mathrm{H}$ & -1457.961997 & -1269.509354 & -188.436222 & -0.016421 & -10.30434171 \\
\hline & $\mathrm{G}$ & -1458.024245 & -1269.557488 & -188.460545 & -0.006212 & -3.8980921201 \\
\hline $\mathrm{V}$ & $\mathrm{U}$ & -1471.2375 & -1282.790671 & -188.43982 & -0.007009 & -4.39821759 \\
\hline \multirow[t]{3}{*}{5} & U+therm & -1471.223362 & -1282.780859 & -188.437166 & -0.005337 & -3.34902087 \\
\hline & $\mathrm{H}$ & -1471.222418 & -1282.779915 & -188.436222 & -0.006281 & -3.94139031 \\
\hline & $\mathrm{G}$ & -1471.284912 & -1282.829429 & -188.460545 & 0.005062 & 3.1764556198 \\
\hline $\mathrm{Cr}$ & $\mathrm{U}$ & -1486.236775 & -1297.785394 & -188.43982 & -0.011561 & -7.25464311 \\
\hline \multirow[t]{3}{*}{6} & $\mathrm{U}+$ therm & -1486.222523 & -1297.775552 & -188.437166 & -0.009805 & -6.1527355499 \\
\hline & $\mathrm{H}$ & -1486.221579 & -1297.774608 & -188.436222 & -0.010749 & -6.74510499 \\
\hline & $\mathrm{G}$ & -1486.285379 & -1297.824184 & -188.460545 & -0.00065 & -0.4078815 \\
\hline Mn & $\mathrm{U}$ & -1503.81344 & -1315.366739 & -188.43982 & -0.006881 & -4.3178963099 \\
\hline \multirow{3}{*}{7} & U+therm & -1503.799124 & -1315.356757 & -188.437166 & -0.005201 & -3.26367951 \\
\hline & $\mathrm{H}$ & -1503.798179 & -1315.355813 & -188.436222 & -0.006144 & -3.85542144 \\
\hline & $\mathrm{G}$ & -1503.863188 & -1315.406167 & -188.460545 & 0.003524 & 2.21134524 \\
\hline $\mathrm{Fe}$ & $\mathrm{U}$ & -1523.376682 & -1334.926102 & $-188,43982$ & & \\
\hline \multirow[t]{3}{*}{4} & U+therm & -1523.362519 & -1334.916206 & -188.437166 & -0.009147 & -5.73983397 \\
\hline & $\mathrm{H}$ & -1523.361575 & -1334.915262 & -188.436222 & -0.010091 & -6.3322034099 \\
\hline & $\mathrm{G}$ & -1523.423996 & -1334.965189 & -188.460545 & 0.001738 & 1.09061238 \\
\hline $\mathrm{Co}$ & $\mathrm{U}$ & -1545.020676 & -1356.570275 & -188.43982 & -0.010581 & -6.63968331 \\
\hline \multirow[t]{3}{*}{3} & U+therm & -1545.007574 & -1356.560395 & -188.437166 & -0.010013 & -6.28325763 \\
\hline & $\mathrm{H}$ & -1545.00663 & -1356.559451 & -188.436222 & -0.010957 & -6.87562707 \\
\hline & $\mathrm{G}$ & -1545.065111 & -1356.609145 & -188.460545 & 0.004579 & 2.87336829 \\
\hline & & & & & & \\
\hline \multirow[t]{3}{*}{2} & $\frac{\mathrm{U}}{\mathrm{U}+\text { therm }}$ & $\frac{-1569.284633}{-1569.270647}$ & $\frac{-1380.835025}{-1380.825393}$ & $\begin{array}{c}-188.43982 \\
-188.437166\end{array}$ & $\begin{array}{r}-0.009788 \\
-0.008088\end{array}$ & $\frac{-6.1420678799}{-5.07530088}$ \\
\hline & $\mathrm{H}$ & -1569.269703 & -1380.824449 & -188.436222 & -0.009032 & -5.66767032 \\
\hline & $\mathrm{G}$ & -1569.330463 & -1380.872144 & -188.460545 & 0.002226 & 1.3968372599 \\
\hline $\mathrm{Cu}$ & $\mathrm{U}$ & -1596.101251 & -1407.654833 & -188.43982 & -0.006598 & -4.14031098 \\
\hline \multirow[t]{3}{*}{1} & U+therm & -1596.087767 & -1407.644933 & -188.437166 & -0.005668 & -3.55672668 \\
\hline & $\mathrm{H}$ & -1596.086823 & -1407.643989 & -188.436222 & -0.006612 & -4.14909612 \\
\hline & $\mathrm{G}$ & -1596.146455 & -1407.692499 & -188.460545 & 0.006589 & 4.13466339 \\
\hline $\mathrm{Zn}$ & $\mathrm{U}$ & -1465.836196 & -1277.392125 & -188.43982 & -0.004251 & -2.66754501 \\
\hline \multirow[t]{3}{*}{2} & U+therm & -1465.821406 & -1277.381908 & -188.437166 & -0.002332 & -1.46335332 \\
\hline & $\mathrm{H}$ & -1465.820462 & -1277.380964 & -188.436222 & -0.003276 & -2.05572276 \\
\hline & $\mathrm{G}$ & -1465.88664 & -1277.432125 & -188.460545 & 0.00603 & 3.7838853 \\
\hline
\end{tabular}


(iii)Table S9:Parallel Binding Mode(M06-2X)

\begin{tabular}{|c|c|c|c|c|c|c|}
\hline Dopant & Parameters & Cluster+CO2 & doped cluster & $\mathrm{CO} 2$ & Delta(au) & in $\mathrm{kcal} / \mathrm{mol}$ \\
\hline \multirow[t]{2}{*}{ Sc } & $\mathrm{U}$ & -1447.017672 & -1258.386728 & -188.558113 & -0.072831 & -45.70218081 \\
\hline & U+therm & -1447.005394 & -1258.377257 & -188.555511 & -0.072626 & -45.57354126 \\
\hline \multirow[t]{2}{*}{3} & $\mathrm{H}$ & -1447.00445 & -1258.376313 & -188.554567 & -0.07357 & -46.1659107 \\
\hline & $\bar{G}$ & -1447.05813 & -1258.423839 & -188.578788 & -0.055503 & -34.82868753 \\
\hline \multirow{3}{*}{$\mathrm{Ti}$} & & & & & & \\
\hline & $\mathrm{U}$ & -1458.612041 & -1269.992237 & -188.558113 & -0.061691 & -38.71171941 \\
\hline & U+therm & -1458.600108 & -1269.983182 & -188.555511 & -0.061415 & -38.53852665 \\
\hline \multirow[t]{2}{*}{4} & $\mathrm{H}$ & -1458.599164 & -1269.982238 & -188.554567 & -0.062359 & -39.13089609 \\
\hline & $\mathrm{G}$ & -1458.652798 & -1270.030177 & -188.578788 & -0.043833 & -27.50564583 \\
\hline \multirow{2}{*}{ V } & U & -1471870111 & -1283266212 & -188558113 & -0045786 & -2873117286 \\
\hline & U+therm & -1471.857692 & -1283.256705 & -188.555511 & -0.045476 & -28.53664476 \\
\hline \multirow[t]{2}{*}{5} & $\mathrm{H}$ & -1471.856748 & -1283.255761 & -188.554567 & -0.04642 & -29.1290142 \\
\hline & $\mathrm{G}$ & -1471.911805 & -1283.304383 & -188.578788 & -0.028634 & -17.96812134 \\
\hline \multirow{3}{*}{$\mathrm{Cr}$} & & & & & & \\
\hline & $\mathrm{U}$ & -1486.901659 & -1298.310167 & -188.558113 & -0.033379 & -20.94565629 \\
\hline & U+therm & -1486.889105 & -1298.300584 & -188.555511 & -0.03301 & -20.7141051 \\
\hline \multirow[t]{2}{*}{6} & $\mathrm{H}$ & -1486.888161 & -1298.29964 & -188.554567 & -0.033954 & -21.30647454 \\
\hline & $\mathrm{G}$ & -1486.943825 & -1298.348617 & -188.578788 & -0.01642 & -10.3037142 \\
\hline \multirow[t]{2}{*}{ Mn } & $\overline{\mathrm{U}}$ & -1504.527807 & -1315.92768 & -188.558113 & -0.042014 & -26.36420514 \\
\hline & U+therm & -1504.515229 & -1315.91796 & -188.555511 & -0.041758 & -26.20356258 \\
\hline \multirow[t]{2}{*}{7} & $\mathrm{H}$ & -1504.514285 & -1315.917016 & -188.554567 & -0.042702 & -26.79593202 \\
\hline & $\mathrm{G}$ & -1504.569976 & -1315.96646 & -188.578788 & -0.024728 & -15.51706728 \\
\hline \multirow[t]{2}{*}{$\mathrm{Fe}$} & $\overline{\mathrm{U}}$ & -1524.024963 & -1335.430574 & -188.558113 & -0.036276 & -22.76355276 \\
\hline & U+therm & -1524.012361 & -1335.421391 & -188.555511 & -0.035459 & -22.25087709 \\
\hline \multirow[t]{2}{*}{4} & $\mathrm{H}$ & -1524.011417 & -1335.420447 & -188.554567 & -0.036403 & -22.84324653 \\
\hline & $\mathrm{G}$ & -1524.066609 & -1335.468827 & -188.578788 & -0.018994 & -11.91892494 \\
\hline \multirow{3}{*}{$\mathrm{Co}$} & & & & & & \\
\hline & $\overline{\mathrm{U}}$ & -1545.680931 & -1357.095494 & -188.558113 & -0.027324 & -17.14608324 \\
\hline & U+therm & -1545.66809 & -1357.085983 & -188.555511 & -0.026596 & -16.68925596 \\
\hline \multirow[t]{2}{*}{3} & $\mathrm{H}$ & -1545.667146 & -1357.085039 & -188.554567 & -0.02754 & -17.2816254 \\
\hline & $\mathrm{G}$ & -1545.724453 & -1357.133078 & -188.578788 & -0.012587 & -7.89846837 \\
\hline \multirow[t]{2}{*}{$\mathrm{Ni}$} & $\mathrm{U}$ & -1569.936211 & -1381.355267 & -188.558113 & -0.022831 & -14.32668081 \\
\hline & U+therm & -1569.923646 & -1381.345789 & -188.555511 & -0.022346 & -14.02233846 \\
\hline \multirow[t]{2}{*}{2} & $\mathrm{H}$ & -1569.922702 & -1381.344844 & -188.554567 & -0.023291 & -14.61533541 \\
\hline & $\mathrm{G}$ & -1569.977115 & -1381.392372 & -188.578788 & -0.005955 & -3.7368220499 \\
\hline \multirow[t]{2}{*}{$\mathrm{Cu}$} & $\overline{\mathrm{U}}$ & -1596.82588 & -1408.24351 & -188.558113 & -0.024257 & -15.22151007 \\
\hline & U+therm & -1596.813336 & -1408.23399 & -188.555511 & -0.023835 & -14.95670085 \\
\hline \multirow[t]{2}{*}{1} & $\mathrm{H}$ & -1596.812392 & -1408.233046 & -188.554567 & -0.024779 & -15.54907029 \\
\hline & $\mathrm{G}$ & -1596.866381 & -1408.280101 & -188.578788 & -0.007492 & -4.70130492 \\
\hline \multirow[t]{2}{*}{$\mathrm{Zn}$} & $\overline{\mathrm{U}}$ & -1466.042859 & -1277.460165 & -188.558113 & -0.024581 & -15.42482331 \\
\hline & U+therm & -1466.02985 & -1277.449931 & -188.555511 & -0.024408 & -15.31626408 \\
\hline \multirow[t]{2}{*}{2} & $\mathrm{H}$ & -1466.028906 & -1277.448987 & -188.554567 & -0.025352 & -15.90863352 \\
\hline & $\mathrm{G}$ & -1466.086593 & -1277.499927 & -188.578788 & -0.007878 & -4.9435237799 \\
\hline
\end{tabular}


(iv)Table S10:Parallel Binding Mode(PBE-PBE)

\begin{tabular}{|c|c|c|c|c|c|c|}
\hline Dopant & Parameters & Cluster+CO2 & doped cluster & $\mathrm{CO} 2$ & Delta(in a.u.) & in $\mathrm{kcal} / \mathrm{mol}$ \\
\hline Sc & $\mathrm{U}$ & -1446.422589 & -1257.916165 & -188.43982 & -0.066604 & -41.79467604 \\
\hline \multirow[t]{3}{*}{3} & U+therm & -1446.410078 & -1257.906823 & -188.437166 & -0.066089 & -41.47150839 \\
\hline & $\mathrm{H}$ & -1446.409134 & -1257.905879 & -188.436222 & -0.067033 & -42.06387783 \\
\hline & $\mathrm{G}$ & -1446.463425 & -1257.952818 & -188.460545 & -0.050062 & -31.41440562 \\
\hline Ti & $\mathrm{U}$ & -1458.031779 & -1269.528604 & -188.43982 & -0.063355 & -39.75589605 \\
\hline \multirow[t]{3}{*}{4} & U+therm & -1458.019279 & -1269.51887 & -188.437166 & -0.063243 & -39.68561493 \\
\hline & $\mathrm{H}$ & -1458.018335 & -1269.517926 & -188.436222 & -0.064187 & -40.27798437 \\
\hline & $\mathrm{G}$ & -1458.072953 & -1269.567432 & -188.460545 & -0.044976 & -28.22288976 \\
\hline $\mathrm{V}$ & & -1471.278887 & -1282.790671 & -188.43982 & -0.048396 & -30.36897396 \\
\hline \multirow[t]{3}{*}{5} & U+therm & -1471.266408 & -1282.780859 & -188.437166 & -0.048383 & -30.36081633 \\
\hline & $\mathrm{H}$ & -1471.265464 & -1282.779915 & -188.436222 & -0.049327 & -30.95318577 \\
\hline & $\mathrm{G}$ & -1471.320343 & -1282.829429 & -188.460545 & -0.030369 & -19.05685119 \\
\hline $\mathrm{Cr}$ & $\mathrm{U}$ & -1486.256516 & -1297.785394 & -188.43982 & -0.031302 & -19.64231802 \\
\hline \multirow[t]{3}{*}{6} & U+therm & -1486.243669 & -1297.775552 & -188.437166 & -0.030951 & -19.42206201 \\
\hline & $\mathrm{H}$ & -1486.242724 & -1297.774608 & -188.436222 & -0.031894 & -20.01380394 \\
\hline & $\mathrm{G}$ & -1486.299265 & -1297.824184 & -188.460545 & -0.014536 & -9.1214853601 \\
\hline Mn & $\mathrm{U}$ & -1503.862137 & -1315.387716 & -188.43982 & -0.034601 & -21.71247351 \\
\hline \multirow[t]{3}{*}{7} & U+therm & -1503.849135 & -1315.377944 & -188.437166 & -0.034025 & -21.35102775 \\
\hline & $\mathrm{H}$ & -1503.848191 & -1315.376999 & -188.436222 & -0.03497 & -21.9440247 \\
\hline & $\mathrm{G}$ & -1503.905075 & -1315.426638 & -188.460545 & -0.017892 & -11.22740892 \\
\hline $\mathrm{Fe}$ & $\mathrm{U}$ & -1523.397147 & -1334.926102 & -188.43982 & -0.031225 & -19.59399975 \\
\hline \multirow[t]{3}{*}{4} & U+therm & -1523.384159 & -1334.916206 & -188.437166 & -0.030787 & -19.31915037 \\
\hline & $\mathrm{H}$ & -1523.383215 & -1334.915262 & -188.436222 & -0.031731 & -19.91151981 \\
\hline & G & -1523.439642 & -1334.965189 & -188.460545 & -0.013908 & -8.7274090801 \\
\hline $\mathrm{Co}$ & $\mathrm{U}$ & -1545.0566 & -1356.593955 & -188.43982 & -0.022825 & -14.32291575 \\
\hline \multirow[t]{3}{*}{3} & U+therm & -1545.043585 & -1356.584252 & -188.437166 & -0.022167 & -13.91001417 \\
\hline & $\mathrm{H}$ & -1545.042641 & -1356.583308 & -188.436222 & -0.023111 & -14.50238361 \\
\hline & $\mathrm{G}$ & -1545.09885 & -1356.631423 & -188.460545 & -0.006882 & -4.3185238201 \\
\hline $\mathrm{Ni}$ & $\mathrm{U}$ & -1569.299695 & -1380.835025 & -188.43982 & -0.02485 & -15.5936235 \\
\hline \multirow[t]{3}{*}{2} & U+therm & -1569.286759 & -1380.825393 & -188.437166 & -0.0242 & -15.185742 \\
\hline & $\mathrm{H}$ & -1569.285814 & -1380.824449 & -188.436222 & -0.025143 & -15.77748393 \\
\hline & G & -1569.341433 & -1380.872144 & -188.460545 & -0.008744 & -5.4869474401 \\
\hline $\mathrm{Cu}$ & $\mathrm{U}$ & -1596.116721 & -1407.654833 & -188.43982 & -0.022068 & -13.84789068 \\
\hline \multirow[t]{3}{*}{1} & U+therm & -1596.103948 & -1407.644933 & -188.437166 & -0.021849 & -13.71046599 \\
\hline & $\mathrm{H}$ & -1596.103004 & -1407.643989 & -188.436222 & -0.022793 & -14.30283543 \\
\hline & $\mathrm{G}$ & -1596.157499 & -1407.692499 & -188.460545 & -0.004455 & -2.79555705 \\
\hline $\mathrm{Zn}$ & U & -1465.850092 & -1277.392125 & -188.43982 & -0.018147 & -11.38742397 \\
\hline \multirow[t]{3}{*}{2} & U+therm & -1465.836924 & -1277.381908 & -188.437166 & -0.01785 & -11.2010535 \\
\hline & $\mathrm{H}$ & -1465.83598 & -1277.380964 & -188.436222 & -0.018794 & -11.79342294 \\
\hline & G & -1465.893753 & -1277.432125 & -188.460545 & -0.001083 & -0.6795933301 \\
\hline
\end{tabular}




\section{Thermochemical Data of $\mathrm{CO}_{2}$ adsorption on $\mathrm{Al}_{7} \mathrm{M}(\mathrm{a})$ clusters}

(i)Table S11: Linear Binding Mode(M06-2X)

\begin{tabular}{|c|c|c|c|c|c|c|}
\hline Dopant & Parameters & Cluster+CO2 & doped cluster & $\mathrm{CO} 2$ & Delta(au) & in $\mathrm{kcal} / \mathrm{mol}$ \\
\hline Sc & $\mathrm{U}$ & & -1743.289247 & -188.558113 & & \\
\hline \multirow[t]{3}{*}{3} & U+therm & no binding & -1743.277135 & -188.555511 & & \\
\hline & $\mathrm{H}$ & found & -1743.276191 & -188.554567 & & \\
\hline & $\mathrm{G}$ & & -1743.329393 & -188.578788 & & \\
\hline $\mathrm{Ti}$ & $\mathrm{U}$ & -1943.476152 & -1754.895107 & -188558113 & -0.022932 & \\
\hline \multirow[t]{3}{*}{4} & U+therm & -1943.460515 & -1754.882437 & -188.555511 & -0.022567 & -14.16101817 \\
\hline & $\mathrm{H}$ & -1943.459571 & -1754.881492 & -188.554567 & -0.023512 & -14.7540151201 \\
\hline & $G$ & -1943.522729 & -1754.935737 & -188.578788 & -0.008204 & -5.14809204 \\
\hline $\mathrm{V}$ & $\mathrm{U}$ & -1956.747811 & -1768.168243 & -188.558113 & -0.021455 & -13.4632270499 \\
\hline \multirow[t]{3}{*}{5} & $\mathrm{U}+$ therm & -1956.73126 & -1768.155624 & -188.555511 & -0.020125 & -12.62863875 \\
\hline & $\mathbf{H}$ & -1956.730316 & -1768.15468 & -188.554567 & -0.021069 & -13.2210081899 \\
\hline & $G$ & -1956.79627 & -1768.2093 & -188.578788 & -0.008182 & -5.13428682 \\
\hline $\mathrm{Cr}$ & $\mathrm{U}$ & -1971.790666 & -1783.2161 & -188.558113 & -0.016453 & -10.32442203 \\
\hline \multirow[t]{3}{*}{6} & $\mathrm{U}+$ therm & -1971.773849 & -1783.203505 & -188.555511 & -0.014833 & -9.30785583 \\
\hline & $\mathrm{H}$ & -1971.772905 & -1783.20256 & -188.554567 & -0.015778 & -9.9008527801 \\
\hline & $G$ & -1971.840902 & -1783.257447 & -188.578788 & -0.004667 & -2.92858917 \\
\hline Mn & $\mathrm{U}$ & -1989.328244 & -1800.832759 & -188.558113 & 0.062628 & 39.2996962799 \\
\hline \multirow[t]{3}{*}{5} & U+therm & -1989.312234 & -1800.820405 & -188.555511 & 0.063682 & 39.96109182 \\
\hline & $\mathrm{H}$ & -1989.31129 & -1800.819461 & -188.554567 & 0.062738 & 39.3687223799 \\
\hline & $G$ & -1989.377035 & -1800.873174 & -188.578788 & 0.074927 & 47.0174417701 \\
\hline $\mathrm{Fe}$ & $\mathrm{U}$ & -2008.916798 & -1820.333425 & -188.558113 & -0.02526 & -15.8509025999 \\
\hline \multirow[t]{3}{*}{4} & $\mathrm{U}+$ therm & -2008.901078 & -1820.320853 & -188.555511 & -0.024714 & -15.5082821401 \\
\hline & $\mathrm{H}$ & -2008.900134 & -1820.319909 & -188.554567 & -0.025658 & -16.10065158 \\
\hline & $\mathrm{G}$ & -2008.964105 & -1820.374908 & -188.578788 & -0.010409 & -6.53175159 \\
\hline Co & $\mathrm{U}$ & -2030.562695 & -1841.991177 & -188.558113 & -0.013405 & -8.41177155 \\
\hline \multirow[t]{3}{*}{3} & $\mathrm{U}+$ therm & -2030.545947 & -1841.978563 & -188.555511 & -0.011873 & -7.4504262301 \\
\hline & $\mathrm{H}$ & -2030.545003 & -1841.977618 & -188.554567 & -0.012818 & -8.0434231801 \\
\hline & $G$ & -2030.61256 & -1842.032542 & -188.578788 & -0.00123 & -0.7718373 \\
\hline $\mathrm{Ni}$ & $\mathrm{U}$ & -2054.822428 & -1866.252289 & -188.558113 & -0.012026 & -7.5464352599 \\
\hline \multirow[t]{3}{*}{2} & U+therm & -2054.80646 & -1866.239674 & -188.555511 & -0.011275 & -7.0751752499 \\
\hline & $\mathrm{H}$ & -2054.805516 & -1866.238729 & -188.554567 & -0.01222 & -7.6681722 \\
\hline & $G$ & -2054.871341 & -1866.293262 & -188.578788 & 0.000709 & 0.4449045899 \\
\hline $\mathrm{Cu}$ & $\mathrm{U}$ & -2081.713065 & -1893.144947 & -188.558113 & -0.010005 & -6.2782375501 \\
\hline \multirow[t]{3}{*}{1} & $\mathrm{U}+$ therm & -2081.695981 & -1893.132332 & -188.555511 & -0.008138 & -5.1066763801 \\
\hline & $\mathrm{H}$ & -2081.695036 & -1893.131388 & -188.554567 & -0.009081 & -5.6984183101 \\
\hline & $\mathrm{G}$ & -2081.764808 & -1893.185319 & -188.578788 & -0.000701 & -0.43988451 \\
\hline $\mathrm{Zn}$ & $\mathrm{U}$ & -1950.922598 & -1762.357087 & -188.558113 & -0.007398 & -4.64231898 \\
\hline \multirow[t]{3}{*}{2} & $\mathrm{U}+$ therm & -1950.905997 & -1762.344857 & -188.555511 & -0.005629 & -3.53225379 \\
\hline & $\mathrm{H}$ & -1950.905053 & -1762.343912 & -188.554567 & -0.006574 & -4.12525074 \\
\hline & G & -1950.97271 & -1762.398953 & -188.578788 & 0.005031 & 3.1570028099 \\
\hline
\end{tabular}


(ii)Table S12: Linear Binding Mode(PBE-PBE)

\begin{tabular}{|c|c|c|c|c|c|c|}
\hline Dopant & Parameters & Cluster+CO2 & doped cluster & $\mathrm{CO} 2$ & Delta(au) & in $\mathrm{kcal} / \mathrm{mol}$ \\
\hline Sc & $\mathrm{U}$ & & -1742.586414 & -188.43982 & & \\
\hline \multirow[t]{3}{*}{3} & $\mathrm{U}+$ therm & no binding & -1742.57404 & -188.437166 & & \\
\hline & $\mathrm{H}$ & found & -1742.573096 & -188.436222 & & \\
\hline & $\mathrm{G}$ & & -1742.626916 & -188.460545 & & \\
\hline & & & & & & \\
\hline $\mathrm{Ti}$ & $\mathrm{U}$ & -1942.661274 & -1754.204965 & -188.43982 & -0.016489 & -10.3470123901 \\
\hline \multirow[t]{3}{*}{4} & U+therm & -1942.644483 & -1754.192535 & -188.437166 & -0.014782 & -9.2758528201 \\
\hline & $\mathrm{H}$ & -1942.643539 & -1754.191591 & -188.436222 & -0.015726 & -9.8682222599 \\
\hline & $\mathrm{G}$ & -1942.711092 & -1754.244768 & -188.460545 & -0.005779 & -3.62638029 \\
\hline $\bar{V}$ & IT & olnding & & & & \\
\hline \multirow[t]{3}{*}{5} & U+therm & found & -1767.447613 & -188.437166 & & \\
\hline & $\mathrm{H}$ & & -1767.446669 & -188.436222 & & \\
\hline & G & & -1767.501272 & -188.460545 & & \\
\hline $\mathrm{Cr}$ & $\mathrm{U}$ & $\begin{array}{l}-1970.918677 \\
\end{array}$ & $-1782,467041$ & -188.43982 & -0.011816 & -74146581598 \\
\hline \multirow[t]{3}{*}{6} & U+therm & -1970.901585 & -1782.4543 & -188.437166 & -0.010119 & -6.34977369 \\
\hline & $\mathrm{H}$ & -1970.900641 & -1782.453356 & -188.436222 & -0.011063 & -6.94214313 \\
\hline & $\mathrm{G}$ & -1970.969276 & -1782.508347 & -188.460545 & -0.000384 & -0.2409638401 \\
\hline Mn & $\mathrm{U}$ & -1988.531339 & -1800.078596 & -188.43982 & -0.012923 & -8.1093117299 \\
\hline \multirow[t]{3}{*}{5} & U+therm & -1988.514398 & -1800.065964 & -188.437166 & -0.011268 & $\begin{array}{l}-7.0707826801 \\
\end{array}$ \\
\hline & $\mathrm{H}$ & -1988.513454 & -1800.06502 & -188.436222 & -0.012212 & -7.6631521199 \\
\hline & $\mathrm{G}$ & -1988.581446 & -1800.11937 & -188.460545 & -0.001531 & -0.9607178101 \\
\hline $\mathrm{Fe}$ & $\mathrm{U}$ & -2008.05745 & -1819.593022 & -188.43982 & -0.024608 & -15.44176608 \\
\hline \multirow[t]{3}{*}{4} & U+therm & -2008.040611 & -1819.580258 & -188.437166 & -0.023187 & $\begin{array}{l}-14.55007437 \\
\end{array}$ \\
\hline & $\mathrm{H}$ & -2008.039667 & -1819.579314 & -188.436222 & -0.024131 & -15.1424438099 \\
\hline & $\mathrm{G}$ & -2008.107701 & -1819.634598 & -188.460545 & -0.012558 & -7.8802705799 \\
\hline $\mathrm{Co}$ & $\mathrm{U}$ & -2029.693035 & -1841.241254 & -188.43982 & -0.011961 & -7.50564711 \\
\hline \multirow[t]{3}{*}{3} & U+therm & -2029.675999 & -1841.228405 & -188.437166 & -0.010428 & -6.54367428 \\
\hline & $\mathrm{H}$ & -2029.675054 & -1841.227461 & -188.436222 & -0.011371 & -7.13541621 \\
\hline & $\mathrm{G}$ & -2029.743688 & -1841.282994 & -188.460545 & -0.000149 & -0.0934989901 \\
\hline $\mathrm{Ni}$ & $\mathrm{U}$ & -2053.954042 & $\mid-1865.503327$ & -188.43982 & -0.010895 & -6.8367214499 \\
\hline \multirow[t]{3}{*}{2} & $\mathrm{U}+$ therm & $\mid-2053.937338$ & -1865.491011 & -188.437166 & -0.009161 & -5.74861911 \\
\hline & $\mathrm{H}$ & -2053.936394 & -1865.490066 & -188.436222 & -0.010106 & -6.3416160598 \\
\hline & G & -2054.003891 & -1865.543788 & -188.460545 & 0.000442 & 0.27735942 \\
\hline $\mathrm{Cu}$ & $\bar{U}$ & $\mid 2080.778278$ & -1892.331838 & -18843982 & -0.00662 & -41541161998 \\
\hline \multirow[t]{3}{*}{1} & U+therm & -2080.761826 & -1892.319052 & -188.437166 & -0.005608 & -3.51907608 \\
\hline & $\mathrm{H}$ & -2080.760881 & -1892.318108 & -188.436222 & -0.006551 & -4.1108180103 \\
\hline & $G$ & -2080.82702 & -1892.372326 & -188.460545 & 0.005851 & 3.6715610099 \\
\hline $\mathrm{Zn}$ & $\mathrm{U}$ & -1950.512099 & -1762.067805 & -188.43982 & -0.004474 & -2.80747974 \\
\hline \multirow[t]{3}{*}{2} & $\mathrm{U}+$ therm & -1950.494394 & -1762.054672 & -188.437166 & -0.002556 & -1.6039155601 \\
\hline & $\mathrm{H}$ & -1950.49345 & -1762.053728 & -188.436222 & -0.0035 & -2.1962849999 \\
\hline & $\mathrm{G}$ & -1950.564906 & -1762.110831 & -188.460545 & 0.00647 & 4.0599896999 \\
\hline
\end{tabular}


(iii)Table S13:Parallel Binding Mode(M06-2X)

\begin{tabular}{|c|c|c|c|c|c|c|}
\hline Dopant & Parameters & Cluster+CO2 & doped cluster & $\mathrm{CO} 2$ & Delta(a.u.) & in $\mathrm{kcal} / \mathrm{mol}$ \\
\hline \multirow[t]{2}{*}{ Sc } & $\mathrm{U}$ & -1931.927104 & -1743.29357 & -188.558113 & -0.075421 & -47.32743171 \\
\hline & U+therm & -1931.912792 & -1743.281061 & -188.555511 & -0.07622 & -47.8288122 \\
\hline \multirow[t]{2}{*}{3} & $\mathrm{H}$ & -1931.911848 & -1743.280117 & -188.554567 & -0.077164 & -48.42118164 \\
\hline & G & -1931.969729 & -1743.334712 & -188.578788 & -0.056229 & -35.28425979 \\
\hline \multirow[t]{2}{*}{$\mathrm{Ti}$} & $\mathrm{U}$ & -1943.523961 & -1754.90368 & -188.558113 & -0.062168 & -39.01104168 \\
\hline & U+therm & -1943.509186 & -1754.891865 & -188.555511 & -0.06181 & -38.7863931 \\
\hline \multirow[t]{2}{*}{4} & $\mathrm{H}$ & -1943.508242 & -1754.890921 & -188.554567 & -0.062754 & -39.37876254 \\
\hline & G & -1943.567333 & -1754.943765 & -188.578788 & -0.04478 & -28.0998978 \\
\hline \multirow[t]{2}{*}{$\mathrm{V}$} & $\mathrm{U}$ & -1956.783074 & -1768.173672 & -188.558113 & -0.051289 & -32.18436039 \\
\hline & U+therm & -1956.768544 & -1768.161291 & -188.555511 & -0.051742 & -32.46862242 \\
\hline \multirow[t]{2}{*}{5} & $\mathrm{H}$ & -1956.7676 & -1768.160347 & -188.554567 & -0.052686 & -33.06099186 \\
\hline & $G$ & -1956.826526 & -1768.214818 & -188.578788 & -0.03292 & -20.6576292 \\
\hline \multirow{2}{*}{$\mathrm{Cr}$} & $\mathrm{U}$ & -1971.805768 & -1783.219501 & -188.558113 & -0.028154 & -17.66691654 \\
\hline & U+therm & -1971.789993 & -1783.207 & -188.555511 & -0.027482 & -17.24522982 \\
\hline \multirow[t]{2}{*}{6} & $\mathrm{H}$ & -1971.789049 & -1783.206056 & -188.554567 & -0.028426 & -17.83759926 \\
\hline & $G$ & -1971.851678 & -1783.261037 & -188.578788 & -0.011853 & -7.43787603 \\
\hline \multirow[t]{2}{*}{$\mathrm{Mn}$} & $\bar{U}$ & -1989.438849 & -1800.836752 & -188.558113 & -0.043984 & -27.60039984 \\
\hline & U+therm & -1989.423566 & -1800.824177 & -188.555511 & -0.043878 & -27.53388378 \\
\hline \multirow[t]{2}{*}{5} & $\mathrm{H}$ & -1989.422622 & -1800.823233 & -188.554567 & -0.044822 & -28.12625322 \\
\hline & $G$ & -1989.483306 & -1800.878253 & -188.578788 & -0.026265 & -16.48155015 \\
\hline \multirow{2}{*}{$\mathrm{Fe}$} & $\overline{\mathrm{U}}$ & -2008931544 & -1820341769 & -188558113 & -0031662 & -1986822162 \\
\hline & U+therm & -2008.916249 & -1820.329361 & -188.555511 & -0.031377 & -19.68938127 \\
\hline \multirow[t]{2}{*}{4} & $\mathrm{H}$ & -2008.915305 & -1820.328417 & -188.554567 & -0.032321 & -20.28175071 \\
\hline & $G$ & -2008.975666 & -1820.382607 & -188.578788 & -0.014271 & -8.95519521 \\
\hline \multirow[t]{2}{*}{ Co } & $\bar{U}$ & -2030.588766 & -1842.002462 & -188.558113 & -0.028191 & -17.69013441 \\
\hline & U+therm & -2030.573193 & -1841.990336 & -188.555511 & -0.027346 & -17.15988846 \\
\hline \multirow[t]{2}{*}{3} & $\mathrm{H}$ & -2030.572249 & -1841.989392 & -188.554567 & -0.02829 & -17.7522579 \\
\hline & G & -2030.633625 & -1842.04262 & -188.578788 & -0.012217 & -7.66628967 \\
\hline \multirow[t]{2}{*}{$\mathrm{Ni}$} & $\mathrm{U}$ & -2054.849745 & -1866.261111 & -188.558113 & $\begin{array}{l}-0.030521 \\
\end{array}$ & -19.15223271 \\
\hline & U+therm & -2054.834178 & -1866.248751 & -188.555511 & -0.029916 & -18.77258916 \\
\hline \multirow[t]{2}{*}{2} & $\mathrm{H}$ & -2054.833234 & -1866.247807 & -188.554567 & -0.03086 & -19.3649586 \\
\hline & $\mathrm{G}$ & -2054.894238 & -1866.301271 & -188.578788 & -0.014179 & -8.8974642899 \\
\hline \multirow[t]{2}{*}{$\mathrm{Cu}$} & $\mathrm{U}$ & -2081.729979 & -1893.152316 & -188.558113 & -0.01955 & -12.2678205 \\
\hline & U+therm & -2081.714311 & -1893.139783 & -188.555511 & -0.019017 & -11.93335767 \\
\hline \multirow[t]{2}{*}{1} & $\mathrm{H}$ & -2081.713367 & -1893.138839 & -188.554567 & -0.019961 & -12.52572711 \\
\hline & G & -2081.774001 & -1893.192471 & -188.578788 & -0.002742 & -1.7206324201 \\
\hline \multirow[t]{2}{*}{$\mathrm{Zn}$} & $\mathrm{U}$ & -1950.932577 & -1762.357087 & -188.558113 & -0.017377 & -10.90424127 \\
\hline & U+therm & -1950.916783 & -1762.344857 & -188.555511 & -0.016415 & -10.30057665 \\
\hline \multirow[t]{2}{*}{2} & $\mathrm{H}$ & -1950.915839 & -1762.343912 & -188.554567 & -0.01736 & -10.8935736 \\
\hline & $\mathrm{G}$ & -1950.977496 & -1762.398953 & -188.578788 & 0.000245 & 0.15373995 \\
\hline
\end{tabular}


(iv)Table S14:Parallel Binding Mode(PBE-PBE)

\begin{tabular}{|c|c|c|c|c|c|c|}
\hline Dopant & Paramets & Cluster+CO2 & doped cluster & $\mathrm{CO} 2$ & Delta(a.u.) & in $\mathrm{kcal} / \mathrm{mol}$ \\
\hline Sc & $\mathrm{U}$ & -1931.102853 & -1742.591234 & -188.43982 & -0.071799 & -45.05459049 \\
\hline \multirow[t]{3}{*}{3} & U+therm & -1931.087572 & -1742.578779 & -188.437166 & -0.071627 & -44.94665877 \\
\hline & $\mathrm{H}$ & -1931.086628 & -1742.577835 & -188.436222 & -0.072571 & -45.53902821 \\
\hline & G & -1931.146512 & -1742.631514 & -188.460545 & -0.054453 & -34.16980203 \\
\hline Ti & $\bar{U}$ & -1942.708411 & -1754.212792 & -188.43982 & -0.055799 & -35.01443049 \\
\hline \multirow{3}{*}{4} & U+therm & 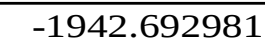 & -1754.200381 & -188.437166 & -0.055434 & -34.78538934 \\
\hline & $\mathrm{H}$ & -1942.692037 & -1754.199437 & -188.436222 & -0.056378 & -35.37775878 \\
\hline & G & -1942.752584 & -1754.253225 & -188.460545 & -0.038814 & -24.35617314 \\
\hline $\mathrm{V}$ & $\mathrm{U}$ & -1955.963375 & -1767.470133 & -188.43982 & -0.053422 & -33.52283922 \\
\hline \multirow[t]{3}{*}{5} & $\mathrm{U}+$ therm & -1955.947945 & -1767.457564 & -188.437166 & -0.053215 & -33.39294465 \\
\hline & $\mathrm{H}$ & -1955.947001 & -1767.45662 & -188.436222 & -0.054159 & -33.98531409 \\
\hline & G & -1956.007904 & -1767.511213 & -188.460545 & -0.036146 & -22.68197646 \\
\hline $\mathrm{Cr}$ & $\mathrm{U}$ & -1970939586 & $-1782,466275$ & $-188,43982$ & -0033491 & -2101593741 \\
\hline \multirow[t]{3}{*}{6} & U+therm & -1970.923747 & -1782.453575 & -188.437166 & -0.033006 & -20.71159506 \\
\hline & $\mathrm{H}$ & -1970.922803 & $\begin{array}{l}-1782.45263 \\
\end{array}$ & -188.436222 & -0.033951 & -21.30459201 \\
\hline & G & -1970.985764 & -1782.507971 & -188.460545 & -0.017248 & -10.82329248 \\
\hline Mn & $\mathrm{U}$ & -1988.553957 & -1800.078332 & $\begin{array}{l}-188.43982 \\
\end{array}$ & -0.035805 & -22.46799555 \\
\hline \multirow[t]{3}{*}{5} & $\mathrm{U}+$ therm & -1988.538114 & -1800.065707 & -188.437166 & -0.035241 & -22.11407991 \\
\hline & $\mathrm{H}$ & -1988.537169 & -1800.064763 & -188.436222 & -0.036184 & -22.70582184 \\
\hline & G & -1988.599286 & -1800.119612 & -188.460545 & -0.019129 & -12.00363879 \\
\hline & & & & & & \\
\hline $\mathrm{Fe}$ & $\mathrm{U}$ & -2008.074662 & -1819.606717 & -188.43982 & -0.028125 & -17.64871875 \\
\hline \multirow[t]{3}{*}{4} & U+therm & -2008.05909 & -1819.594189 & -188.437166 & -0.027735 & -17.40398985 \\
\hline & $\mathrm{H}$ & -2008.058146 & -1819.593245 & -188.436222 & -0.028679 & -17.99635929 \\
\hline & $\mathrm{G}$ & -2008.119035 & -1819.647453 & -188.460545 & -0.011037 & -6.92582787 \\
\hline $\mathrm{Co}$ & $\mathrm{U}$ & -2029.732993 & -1841.265601 & -188.43982 & -0.027572 & -17.30170572 \\
\hline \multirow[t]{3}{*}{3} & $\mathrm{U}+$ therm & -2029.717133 & -1841.253726 & -188.437166 & -0.026241 & -16.46648991 \\
\hline & $\mathrm{H}$ & -2029.716189 & -1841.252782 & -188.436222 & -0.027185 & -17.05885935 \\
\hline & G & -2029.778138 & -1841.305329 & -188.460545 & -0.012264 & -7.69578264 \\
\hline $\mathrm{Ni}$ & $\bar{U}$ & -2053.989677 & -1865.523802 & -188.43982 & -0.026055 & -16.34977305 \\
\hline \multirow[t]{3}{*}{2} & $\mathrm{U}+$ therm & -2053.974028 & -1865.511405 & -188.437166 & -0.025457 & -15.97452207 \\
\hline & $\mathrm{H}$ & -2053.973084 & -1865.510461 & -188.436222 & -0.026401 & -16.56689151 \\
\hline & $\mathrm{G}$ & -2054.033932 & -1865.563988 & -188.460545 & -0.009399 & -5.8979664897 \\
\hline $\mathrm{Cu}$ & $\mathrm{U}$ & -2080.798646 & -1892.337718 & -188.43982 & -0.021108 & -13.24548108 \\
\hline \multirow[t]{3}{*}{1} & $\mathrm{U}+$ therm & -2080.782548 & -1892.324976 & -188.437166 & -0.020406 & -12.80496906 \\
\hline & $\mathrm{H}$ & -2080.781604 & -1892.324032 & -188.436222 & -0.02135 & -13.3973385 \\
\hline & $\mathrm{G}$ & -2080.843519 & -1892.378015 & -188.460545 & -0.004959 & -3.1118220899 \\
\hline $\mathrm{Zn}$ & $\mathrm{U}$ & -1950.527191 & -1762.067805 & -188.43982 & -0.019566 & -12.27786066 \\
\hline \multirow[t]{3}{*}{2} & U+therm & -1950.510925 & -1762.054672 & -188.437166 & -0.019087 & -11.97728337 \\
\hline & $\mathrm{H}$ & -1950.509981 & -1762.053728 & -188.436222 & -0.020031 & -12.56965281 \\
\hline & $G$ & -1950.574039 & -1762.110831 & -188.460545 & $\begin{array}{l}-0.002663 \\
\end{array}$ & -1.6710591301 \\
\hline
\end{tabular}


Thermochemical Data of $\mathrm{CO}_{2}$ adsorption on $\mathrm{Al}_{7} \underline{\mathrm{M}(\mathrm{b}) \text { clusters }}$

(i)Table S15: Linear Binding Mode(M06-2X)

\begin{tabular}{|c|c|c|c|c|c|c|}
\hline Dopant & Parameters & cluster+CO2 & doped cluster & $\mathrm{CO} 2$ & Delta(a.u.) & in $\mathrm{kcal} / \mathrm{mol}$ \\
\hline \multirow[t]{2}{*}{$\mathrm{Sc}$} & $\mathrm{U}$ & -1931.875693 & -1743.294497 & -188.558113 & -0.023083 & -14.4848133299 \\
\hline & U+therm & -1931.859562 & -1743.282111 & -188.555511 & -0.02194 & -13.7675694001 \\
\hline \multirow[t]{2}{*}{3} & $\mathrm{H}$ & -1931.858618 & -1743.281167 & -188.554567 & -0.022884 & -14.35993884 \\
\hline & G & -1931.922982 & -1743.334973 & -188.578788 & -0.009221 & -5.78626971 \\
\hline \multirow[t]{2}{*}{$\mathrm{Ti}$} & $\mathrm{U}$ & -1943.484071 & -1754.903645 & -188.558113 & -0.022313 & -14.00163063 \\
\hline & U+therm & -1943.468119 & -1754.891852 & -188.555511 & -0.020756 & -13.0245975599 \\
\hline \multirow[t]{2}{*}{4} & $\mathrm{H}$ & -1943.467175 & -1754.890907 & -188.554567 & -0.021701 & -13.6175945101 \\
\hline & $\mathrm{G}$ & -1943.532933 & -1754.943693 & -188.578788 & -0.010452 & -6.55873452 \\
\hline \multirow[t]{2}{*}{$\mathrm{V}$} & $\mathrm{U}$ & -1956.754599 & -1768.173594 & -188.558113 & -0.022892 & -14.36495892 \\
\hline & U+therm & -1956.73807 & -1768.161261 & -188.555511 & -0.021298 & -13.3647079801 \\
\hline \multirow[t]{2}{*}{5} & $\mathrm{H}$ & -1956.737126 & -1768.160317 & -188.554567 & -0.022242 & -13.95707742 \\
\hline & G & -1956.805441 & -1768.214447 & -188.578788 & -0.012206 & -7.6593870599 \\
\hline \multirow[t]{2}{*}{$\mathrm{Cr}$} & $\mathrm{U}$ & -1971.795306 & -1783.219461 & -188.558113 & -0.017732 & -11.12700732 \\
\hline & U+therm & -1971.778884 & -1783.206982 & -188.555511 & -0.016391 & -10.2855164101 \\
\hline \multirow[t]{2}{*}{6} & $\mathrm{H}$ & -1971.777939 & -1783.206038 & -188.554567 & -0.017334 & -10.8772583401 \\
\hline & $\mathrm{G}$ & -1971.843728 & -1783.260949 & -188.578788 & -0.003991 & -2.50439241 \\
\hline \multirow[t]{2}{*}{$\mathrm{Mn}$} & $\mathrm{U}$ & -1989.412173 & -1800.836772 & -188.558113 & -0.017288 & -10.8483928799 \\
\hline & $\mathrm{U}+$ therm & -1989.39574 & -1800.824165 & -188.555511 & -0.016064 & -10.0803206399 \\
\hline \multirow[t]{2}{*}{5} & $\mathrm{H}$ & -1989.394796 & -1800.823221 & -188.554567 & -0.017008 & $\begin{array}{l}-10.67269008 \\
\end{array}$ \\
\hline & G & -1989.460656 & -1800.878359 & -188.578788 & -0.003509 & -2.2019325899 \\
\hline \multirow[t]{2}{*}{$\mathrm{Fe}$} & $\mathrm{U}$ & -2008.916693 & -1820.341767 & -188.558113 & -0.016813 & -10.55032563 \\
\hline & U+therm & -2008.90009 & -1820.329392 & -188.555511 & -0.015187 & -9.52999437 \\
\hline \multirow[t]{2}{*}{4} & $\mathrm{H}$ & -2008.899146 & -1820.328448 & -188.554567 & -0.016131 & -10.1223638101 \\
\hline & $\mathrm{G}$ & -2008.96662 & -1820.382517 & -188.578788 & -0.005315 & -3.3352156499 \\
\hline \multirow[t]{2}{*}{ Co } & $\mathrm{U}$ & -2030.575469 & -1842.002932 & -188.558113 & -0.014424 & -9.05120424 \\
\hline & U+therm & -2030.559764 & -1841.990523 & -188.555511 & -0.01373 & -8.6157123001 \\
\hline \multirow[t]{2}{*}{3} & $\mathrm{H}$ & -2030.55882 & -1841.989579 & -188.554567 & -0.014674 & -9.20808174 \\
\hline & $G$ & -2030.622497 & -1842.043565 & -188.578788 & -0.000144 & -0.0903614401 \\
\hline \multirow[t]{2}{*}{$\mathrm{Ni}$} & $\mathrm{U}$ & -2054.806575 & -1866.26126 & -188.558113 & 0.012798 & 8.0308729798 \\
\hline & U+therm & -2054.790441 & -1866.248853 & -188.555511 & 0.013923 & 8.73682173 \\
\hline \multirow[t]{2}{*}{2} & $\mathrm{H}$ & -2054.789497 & -1866.247909 & -188.554567 & 0.012979 & 8.1444522898 \\
\hline & $G$ & -2054.853855 & -1866.301528 & -188.578788 & 0.026461 & 16.60454211 \\
\hline \multirow[t]{2}{*}{$\mathrm{Cu}$} & $\mathrm{U}$ & -2081.718789 & -1893.149157 & -188.558113 & -0.011519 & -7.2282876901 \\
\hline & $\mathrm{U}+$ therm & -2081.702859 & -1893.137535 & -188.555511 & -0.009813 & -6.1577556301 \\
\hline \multirow[t]{2}{*}{1} & $\mathrm{H}$ & -2081.701915 & -1893.136591 & -188.554567 & -0.010757 & -6.75012507 \\
\hline & $G$ & -2081.766854 & -1893.188746 & -188.578788 & 0.00068 & 0.4267068001 \\
\hline \multirow{2}{*}{$\mathrm{Zn}$} & $\mathrm{U}$ & -1950.921053 & -1762.353417 & -188.558113 & -0.009523 & -5.97577773 \\
\hline & U+therm & -1950.904058 & -1762.340509 & -188.555511 & -0.008038 & -5.04392538 \\
\hline \multirow[t]{2}{*}{2} & $\mathrm{H}$ & -1950.903114 & -1762.339565 & -188.554567 & -0.008982 & -5.63629482 \\
\hline & $G$ & -1950.970508 & -1762.395179 & -188.578788 & 0.003459 & 2.17055709 \\
\hline
\end{tabular}


(ii)Table S16: Linear Binding Mode(PBE-PBE)

\begin{tabular}{|c|c|c|c|c|c|c|}
\hline Dopant & Parameters & cluster+CO2 & doped cluster & $\mathrm{CO} 2$ & Delta(a.u.) & in $\mathrm{kcal} / \mathrm{mol}$ \\
\hline Sc & $\mathrm{U}$ & & -1742.591654 & -188.43982 & & \\
\hline \multirow[t]{3}{*}{3} & U+therm & no binding & -1742.579089 & -188.437166 & & \\
\hline & $\mathrm{H}$ & found & -1742.578145 & -188.436222 & & \\
\hline & G & & -1742.632505 & -188.460545 & & \\
\hline $\mathrm{Ti}$ & $\mathrm{U}$ & -1942.669672 & -1754.212788 & -188.43982 & -0.017064 & -10.7078306399 \\
\hline \multirow[t]{3}{*}{4} & U+therm & -1942.652825 & -1754.200375 & -188.437166 & -0.015284 & -9.59086284 \\
\hline & $\mathrm{H}$ & -1942.65188 & -1754.199431 & -188.436222 & -0.016227 & -10.1826047699 \\
\hline & $\mathrm{G}$ & -1942.720778 & -1754.253227 & -188.460545 & -0.007006 & -4.3963350602 \\
\hline $\mathrm{V}$ & $\mathrm{U}$ & -1955.925729 & -1767.470132 & -188.43982 & -0.015777 & -9.90022527 \\
\hline \multirow[t]{3}{*}{5} & U+therm & -1955.908899 & -1767.457565 & -188.437166 & -0.014168 & -8.8905616801 \\
\hline & $\mathrm{H}$ & -1955.907955 & -1767.456621 & -188.436222 & -0.015112 & -9.4829311199 \\
\hline & $G$ & -1955.975722 & -1767.511216 & -188.460545 & -0.003961 & -2.4855671099 \\
\hline $\mathrm{Cr}$ & $\mathrm{U}$ & -1970.919324 & -1782.46627 & -188.43982 & -0.013234 & -8.30446734 \\
\hline \multirow[t]{3}{*}{6} & U+therm & -1970.9024 & -1782.453558 & -188.437166 & -0.011676 & -7.32680676 \\
\hline & $\mathrm{H}$ & -1970.901455 & -1782.452614 & -188.436222 & -0.012619 & -7.9185486899 \\
\hline & G & -1970.968535 & -1782.507982 & -188.460545 & -0.000008 & -0.00502008 \\
\hline Mn & $\mathrm{U}$ & -1988.531035 & -1800.078318 & -188.43982 & -0.012897 & -8.0929964699 \\
\hline \multirow[t]{3}{*}{5} & U+therm & -1988.514134 & -1800.065681 & -188.437166 & -0.011287 & -7.08270537 \\
\hline & $\mathrm{H}$ & -1988.51319 & -1800.064736 & -188.436222 & -0.012232 & -7.6757023199 \\
\hline & G & -1988.580426 & -1800.119621 & -188.460545 & -0.00026 & -0.1631526 \\
\hline $\mathrm{Fe}$ & $\mathrm{U}$ & -2008.05745 & -1819.608831 & -188.43982 & -0.008799 & -5.52146049 \\
\hline \multirow[t]{3}{*}{4} & U+therm & -2008.040611 & -1819.596262 & -188.437166 & -0.007183 & -4.50740433 \\
\hline & $\mathrm{H}$ & -2008.039667 & -1819.595318 & -188.436222 & -0.008127 & -5.09977377 \\
\hline & $G$ & -2008.1077 & -1819.649779 & -188.460545 & 0.002624 & 1.64658624 \\
\hline Co & $\mathrm{U}$ & -2029.714421 & -1841.265599 & -188.43982 & -0.009002 & -5.6488450199 \\
\hline \multirow[t]{3}{*}{3} & U+therm & -2029.698229 & -1841.253705 & -188.437166 & -0.007358 & -4.61721858 \\
\hline & $\mathrm{H}$ & -2029.697285 & -1841.252761 & -188.436222 & -0.008302 & -5.20958802 \\
\hline & G & -2029.762984 & -1841.30538 & -188.460545 & 0.002941 & 1.84550691 \\
\hline $\mathrm{Ni}$ & $\mathrm{U}$ & -2053.97252 & -1865.523802 & -188.43982 & -0.008898 & -5.58358398 \\
\hline \multirow[t]{3}{*}{2} & U+therm & -2053.955735 & -1865.511393 & -188.437166 & -0.007176 & -4.50301176 \\
\hline & $\mathrm{H}$ & -2053.95479 & -1865.510449 & -188.436222 & -0.008119 & -5.0947536898 \\
\hline & G & -2054.022068 & -1865.564015 & -188.460545 & 0.002492 & 1.5637549198 \\
\hline $\mathrm{Cu}$ & $\mathrm{U}$ & -2080.784901 & -1892.334144 & -188.43982 & -0.010937 & -6.86307687 \\
\hline \multirow[t]{3}{*}{1} & U+therm & -2080.767743 & -1892.321105 & -188.437166 & -0.009472 & -5.9437747198 \\
\hline & $\mathrm{H}$ & -2080.766799 & -1892.32016 & -188.436222 & -0.010417 & -6.53677167 \\
\hline & G & -2080.835643 & -1892.375677 & -188.460545 & 0.000579 & 0.36332829 \\
\hline $\mathrm{Zn}$ & $\mathrm{U}$ & -1950.50513 & -1762.058496 & -188.43982 & -0.006814 & -4.2758531399 \\
\hline \multirow[t]{3}{*}{2} & U+therm & -1950.487406 & -1762.045243 & -188.437166 & -0.004997 & -3.13566747 \\
\hline & $\mathrm{H}$ & -1950.486462 & -1762.044299 & -188.436222 & -0.005941 & -3.7280369101 \\
\hline & $G$ & -1950.557324 & -1762.101201 & -188.460545 & 0.004422 & 2.7748492198 \\
\hline
\end{tabular}


(iii)Table S17:Parallel Binding Mode(M06-2X)

\begin{tabular}{|c|c|c|c|c|c|c|}
\hline Dopant & Parameters & Cluster+CO2 & doped cluster & $\mathrm{CO} 2$ & Delta(a.u.) & in $\mathrm{kcal} / \mathrm{mol}$ \\
\hline \multirow[t]{2}{*}{$\mathrm{Sc}$} & $\mathrm{U}$ & -1931.927084 & -1743.294497 & -188.558113 & -0.074474 & -46.73317974 \\
\hline & U+therm & -1931.912793 & -1743.282111 & -188.555511 & -0.075171 & -47.17055421 \\
\hline \multirow[t]{2}{*}{3} & $\mathrm{H}$ & -1931.911849 & -1743.281167 & -188.554567 & -0.076115 & -47.76292365 \\
\hline & G & -1931.96965 & -1743.334973 & -188.578788 & -0.055889 & -35.07090639 \\
\hline \multirow{2}{*}{$\mathrm{Ti}$} & $\mathrm{U}$ & -1943.520475 & -1754903645 & -188558113 & -0.058717 & -36.84550467 \\
\hline & U+therm & -1943.506059 & -1754.891852 & -188.555511 & -0.058696 & -36.83232696 \\
\hline \multirow[t]{2}{*}{4} & $\mathrm{H}$ & -1943.505114 & -1754.890907 & -188.554567 & -0.05964 & -37.4246964 \\
\hline & $\mathrm{G}$ & -1943.563498 & -1754.943693 & -188.578788 & -0.041017 & -25.73857767 \\
\hline \multirow[t]{2}{*}{$\mathrm{V}$} & $\mathrm{U}$ & -1956.783035 & -1768.173594 & -188.558113 & -0.051328 & -32.20883328 \\
\hline & U+therm & -1956.76854 & -1768.161261 & -188.555511 & -0.051768 & -32.48493768 \\
\hline \multirow[t]{2}{*}{5} & $\mathrm{H}$ & -1956.767596 & -1768.160317 & -188.554567 & -0.052712 & -33.07730712 \\
\hline & $\mathrm{G}$ & -1956.826422 & -1768.214447 & -188.578788 & -0.033187 & -20.82517437 \\
\hline \multirow[t]{2}{*}{$\mathrm{Cr}$} & $\mathrm{U}$ & -1971.805771 & -1783.219461 & -188.558113 & -0.028197 & -17.69389947 \\
\hline & U+therm & -1971.790022 & -1783.206982 & -188.555511 & -0.027529 & -17.27472279 \\
\hline \multirow[t]{2}{*}{6} & $\mathrm{H}$ & -1971.789077 & -1783.206038 & -188.554567 & -0.028472 & -17.86646472 \\
\hline & $G$ & -1971.85163 & -1783.260949 & -188.578788 & -0.011893 & -7.4629764301 \\
\hline \multirow[t]{2}{*}{ Mn } & $\mathrm{U}$ & -1989.438913 & -1800.836772 & -188.558113 & -0.044028 & -27.62801028 \\
\hline & U+therm & -1989.42363 & -1800.824165 & -188.555511 & -0.043954 & -27.58157454 \\
\hline \multirow[t]{2}{*}{5} & $\mathrm{H}$ & -1989.422686 & -1800.823221 & -188.554567 & -0.044898 & -28.17394398 \\
\hline & $G$ & -1989.483356 & -1800.878359 & -188.578788 & -0.026209 & -16.44640959 \\
\hline \multirow[t]{2}{*}{$\mathrm{Fe}$} & $\mathrm{U}$ & -2008.93188 & -1820.341767 & -188.558113 & -0.032 & -20.08032 \\
\hline & U+therm & -2008.917255 & -1820.329392 & -188.555511 & -0.032352 & -20.30120352 \\
\hline \multirow[t]{2}{*}{4} & $\mathrm{H}$ & -2008.916311 & -1820.328448 & -188.554567 & -0.033296 & -20.89357296 \\
\hline & $G$ & -2008.975127 & -1820.382517 & -188.578788 & -0.013822 & -8.6734432199 \\
\hline \multirow{2}{*}{$\mathrm{Co}$} & IT & & & 188558112 & 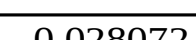 & 1761546077 \\
\hline & $\frac{\mathrm{U}}{\mathrm{U}+\text { therm }}$ & $\frac{-2030.589117}{-2030.574222}$ & -1842.002932 & $\frac{-188.558113}{-188555511}$ & $\frac{-0.028072}{-0028188}$ & $\frac{-17.615460 / 2}{-17.68825188}$ \\
\hline \multirow[t]{2}{*}{3} & $\mathrm{H}$ & -2030.573278 & -1841.989579 & -188.554567 & -0.029132 & -18.28062132 \\
\hline & $\mathrm{G}$ & -2030.632802 & -1842.043565 & -188.578788 & -0.010449 & -6.5568519901 \\
\hline \multirow[t]{2}{*}{$\mathrm{Ni}$} & $\mathrm{U}$ & -2054.849753 & -1866.26126 & -188.558113 & -0.03038 & -19.0637538 \\
\hline & U+therm & -2054.834258 & -1866.248853 & -188.555511 & -0.029894 & -18.75878394 \\
\hline \multirow[t]{2}{*}{2} & $\mathrm{H}$ & -2054.833313 & -1866.247909 & -188.554567 & -0.030837 & -19.35052587 \\
\hline & $G$ & -2054.893978 & -1866.301528 & -188.578788 & -0.013662 & -8.5730416202 \\
\hline \multirow[t]{2}{*}{$\mathrm{Cu}$} & $\mathrm{U}$ & -2081.729975 & -1893.152312 & -188.558113 & -0.01955 & -12.2678205 \\
\hline & U+therm & -2081.714304 & -1893.139799 & -188.555511 & -0.018994 & -11.91892494 \\
\hline \multirow[t]{2}{*}{1} & $\mathrm{H}$ & -2081.71336 & -1893.138855 & -188.554567 & -0.019938 & -12.51129438 \\
\hline & G & -2081.774037 & -1893.192409 & -188.578788 & -0.00284 & -1.7821284001 \\
\hline \multirow{2}{*}{$\mathrm{Zn}$} & IU & 195093261 & 1762353417 & 180558112 & 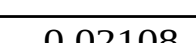 & 132270108 \\
\hline & U+therm & -1950.916784 & -1762.340509 & -188.555511 & -0.020764 & -13.02961764 \\
\hline \multirow[t]{2}{*}{2} & $\mathrm{H}$ & -1950.915839 & -1762.339565 & -188.554567 & -0.021707 & -13.62135957 \\
\hline & $\mathrm{G}$ & -1950.977615 & -1762.395179 & -188.578788 & -0.003648 & -2.2891564799 \\
\hline
\end{tabular}


(iv)Table S18:Parallel Binding Mode(PBE-PBE)

\begin{tabular}{|c|c|c|c|c|c|c|}
\hline Dopant & Parameters & Cluster+CO2 & doped cluster & $\mathrm{CO} 2$ & Delta(a.u.) & in kcal/mol \\
\hline Sc & $\mathrm{U}$ & -1931.102777 & -1742.591654 & -188.43982 & -0.071303 & -44.74334553 \\
\hline \multirow[t]{3}{*}{3} & U+therm & -1931.087486 & -1742.579089 & -188.437166 & -0.071231 & -44.69816481 \\
\hline & $\mathrm{H}$ & -1931.086542 & -1742.578145 & -188.436222 & -0.072175 & -45.29053425 \\
\hline & $G$ & -1931.146464 & -1742.632505 & -188.460545 & -0.053414 & -33.51781914 \\
\hline $\mathrm{Ti}$ & $\mathrm{U}$ & -1942.708434 & -1754.212788 & -188.43982 & -0.055826 & -35.03137326 \\
\hline \multirow[t]{3}{*}{4} & U+therm & -1942.692997 & -1754.200375 & -188.437166 & -0.055456 & -34.79919456 \\
\hline & $\mathrm{H}$ & -1942.692053 & -1754.199431 & -188.436222 & -0.0564 & -35.391564 \\
\hline & $\mathrm{G}$ & -1942.752661 & -1754.253227 & -188.460545 & -0.038889 & -24.40323639 \\
\hline $\mathrm{V}$ & $\mathrm{U}$ & -1955.963404 & -1767.470132 & -188.43982 & -0.053452 & -33.54166452 \\
\hline \multirow[t]{3}{*}{5} & U+therm & -1955.947994 & -1767.457565 & -188.437166 & -0.053263 & -33.42306513 \\
\hline & $\mathrm{H}$ & -1955.94705 & -1767.456621 & -188.436222 & -0.054207 & -34.01543457 \\
\hline & $G$ & -1956.007868 & -1767.511216 & -188.460545 & -0.036107 & -22.65750357 \\
\hline $\mathrm{Cr}$ & $\mathrm{U}$ & -1970.940757 & -1782.46627 & -188.43982 & -0.034667 & -21.75388917 \\
\hline \multirow[t]{3}{*}{6} & U+therm & -1970.925069 & -1782.453558 & -188.437166 & -0.034345 & -21.55183095 \\
\hline & $\mathrm{H}$ & -1970.924124 & -1782.452614 & -188.436222 & -0.035288 & -22.14357288 \\
\hline & $\mathrm{G}$ & -1970.986032 & -1782.507982 & -188.460545 & -0.017505 & -10.98456255 \\
\hline$M n$ & $\mathrm{U}$ & 1988553016 & -1800078318 & 18913087 & 0035778 & -2245105278 \\
\hline \multirow[t]{3}{*}{5} & U+therm & -1988.538046 & -1800.065681 & -188.437166 & -0.035199 & -22.08772449 \\
\hline & $\mathrm{H}$ & -1988.537102 & -1800.064736 & -188.436222 & -0.036144 & -22.68072144 \\
\hline & $\mathrm{G}$ & -1988.599331 & -1800.119621 & -188.460545 & -0.019165 & -12.02622915 \\
\hline $\mathrm{Fe}$ & $\mathrm{U}$ & -2008.079569 & -1819.608831 & -188.43982 & -0.030918 & -19.40135418 \\
\hline \multirow[t]{3}{*}{4} & $\mathrm{U}+$ therm & -2008.063711 & -1819.596262 & -188.437166 & -0.030283 & -19.00288533 \\
\hline & $\mathrm{H}$ & -2008.062767 & -1819.595318 & -188.436222 & -0.031227 & -19.59525477 \\
\hline & $G$ & -2008.125028 & -1819.649779 & -188.460545 & -0.014704 & -9.22690704 \\
\hline Co & $\mathrm{U}$ & -2029.734965 & -1841.265599 & -188.43982 & -0.029546 & -18.54041046 \\
\hline \multirow{3}{*}{3} & U+therm & -2029.719148 & -1841.253705 & -188.437166 & -0.028277 & -17.74410027 \\
\hline & $\mathrm{H}$ & -2029.718204 & -1841.252761 & -188.436222 & -0.029221 & -18.33646971 \\
\hline & $G$ & -2029.78018 & -1841.30538 & -188.460545 & -0.014255 & -8.94515505 \\
\hline $\mathrm{Ni}$ & $\bar{U}$ & -2053989671 & -1865523807 & -18843982 & 0026049 & -1634600799 \\
\hline \multirow[t]{3}{*}{2} & U+therm & -2053.974013 & -1865.511393 & -188.437166 & -0.025454 & -15.97263954 \\
\hline & $\mathrm{H}$ & -2053.973069 & -1865.510449 & -188.436222 & -0.026398 & -16.56500898 \\
\hline & $\mathrm{G}$ & -2054.033948 & -1865.564015 & -188.460545 & -0.009388 & -5.89106388 \\
\hline & & & & & & \\
\hline $\mathrm{Cu}$ & $\mathrm{U}$ & -2080.79866 & -1892.337724 & -188.43982 & -0.021116 & -13.25050116 \\
\hline \multirow[t]{3}{*}{1} & U+therm & -2080.782577 & -1892.324984 & -188.437166 & -0.020427 & -12.81814677 \\
\hline & $\mathrm{H}$ & -2080.781633 & -1892.32404 & -188.436222 & -0.021371 & -13.41051621 \\
\hline & $G$ & -2080.843502 & -1892.378028 & -188.460545 & -0.004929 & -3.0929967901 \\
\hline $7 n$ & $\mathrm{U}$ & 520683 & 8496 & -188.4 & 2367 & 03551617 \\
\hline \multirow[t]{3}{*}{2} & U+therm & -1950.504665 & -1762.045243 & -188.437166 & -0.022256 & -13.96586256 \\
\hline & $\mathrm{H}$ & -1950.503721 & -1762.044299 & -188.436222 & -0.0232 & -14.558232 \\
\hline & $\mathrm{G}$ & -1950.565598 & -1762.101201 & -188.460545 & -0.003852 & -2.41716852 \\
\hline
\end{tabular}


Thermochemical Data of $\mathrm{CO}_{2}$ adsorption on $\mathrm{Al}_{7} \mathrm{M}(\mathrm{c})$ clusters

(i)Table S19: Linear Binding Mode(M06-2X)

\begin{tabular}{|c|c|c|c|c|c|c|}
\hline Dopant & Parameters & Cluster+CO2 & doped cluster & $\mathrm{CO} 2$ & Delta(a.u.) & in $\mathrm{kcal} / \mathrm{mol}$ \\
\hline \multirow[t]{2}{*}{ Sc } & $\mathrm{U}$ & -1931.862803 & -1743.294026 & -188.558113 & -0.010664 & -6.6917666399 \\
\hline & U+therm & -1931.847013 & -1743.282713 & -188.555511 & -0.008789 & -5.51518539 \\
\hline \multirow[t]{2}{*}{3} & $\mathrm{H}$ & -1931.846069 & -1743.281768 & -188.554567 & -0.009734 & -6.10818234 \\
\hline & G & -1931.909994 & -1743.333214 & -188.578788 & 0.002008 & 1.26004008 \\
\hline \multirow[t]{2}{*}{ Ti } & U & Binding not & -1754.86054 & -188.558113 & & \\
\hline & U+therm & found & -1754.847807 & -188.555511 & & \\
\hline \multirow[t]{2}{*}{4} & $\mathrm{H}$ & & -1754.846862 & -188.554567 & & \\
\hline & G & & -1754.902046 & -188.578788 & & \\
\hline \multirow[t]{2}{*}{$\mathrm{V}$} & $\mathrm{U}$ & -1956.754588 & -1768.173594 & -188.558113 & -0.022881 & -14.3580563099 \\
\hline & U+therm & -1956.738068 & -1768.161261 & -188.555511 & -0.021296 & -13.3634529601 \\
\hline \multirow[t]{2}{*}{5} & $\mathrm{H}$ & -1956.737124 & -1768.160317 & -188.554567 & -0.02224 & -13.9558224 \\
\hline & G & -1956.805174 & -1768.214447 & -188.578788 & -0.011939 & -7.49184189 \\
\hline \multirow[t]{2}{*}{$\mathrm{Cr}$} & $\mathrm{U}$ & Binding not & -1783.204125 & -188.558113 & & \\
\hline & U+therm & found & -1783.191625 & -188.555511 & & \\
\hline \multirow[t]{2}{*}{6} & $\mathrm{H}$ & & -1783.190681 & -188.554567 & & \\
\hline & $G$ & & -1783.245599 & -188.578788 & & \\
\hline \multirow[t]{2}{*}{ Mn } & $\mathrm{U}$ & -1989.290703 & -1800.811923 & -188.558113 & 0.079333 & 49.7822508301 \\
\hline & $\mathrm{U}+$ therm & -1989.273953 & -1800.798729 & -188.555511 & 0.080287 & 50.3808953701 \\
\hline \multirow[t]{2}{*}{5} & $\mathrm{H}$ & -1989.273009 & -1800.797785 & -188.554567 & 0.079343 & 49.7885259299 \\
\hline & G & -1989.339874 & -1800.85528 & -188.578788 & 0.094194 & 59.10767694 \\
\hline \multirow[t]{2}{*}{$\mathrm{Fe}$} & $\mathrm{U}$ & -2008.916346 & -1820.34396 & -188.558113 & -0.014273 & -8.95645023 \\
\hline & U+therm & -2008.899765 & -1820.331669 & -188.555511 & -0.012585 & -7.89721335 \\
\hline \multirow[t]{2}{*}{4} & $\mathrm{H}$ & -2008.89882 & -1820.330725 & -188.554567 & -0.013528 & -8.48895528 \\
\hline & $G$ & -2008.965822 & -1820.384534 & -188.578788 & -0.0025 & -1.5687749999 \\
\hline \multirow[t]{2}{*}{$\mathrm{Co}$} & $\mathrm{U}$ & -2030.571984 & -1842.002932 & -188.558113 & -0.010939 & -6.8643318899 \\
\hline & U+therm & -2030.555751 & -1841.990523 & -188.555511 & -0.009717 & -6.0975146701 \\
\hline \multirow[t]{2}{*}{3} & $\mathrm{H}$ & -2030.554807 & -1841.989579 & -188.554567 & -0.010661 & -6.68988411 \\
\hline & $G$ & -2030.620309 & -1842.043565 & -188.578788 & 0.002044 & 1.28263044 \\
\hline \multirow[t]{2}{*}{$\mathrm{Ni}$} & U & -2054.83474 & -1866.26126 & -188.558113 & -0.015367 & -9.64294617 \\
\hline & U+therm & -2054.818999 & -1866.248854 & -188.555511 & -0.014634 & -9.1829813401 \\
\hline \multirow[t]{2}{*}{2} & $\mathrm{H}$ & -2054.818055 & -1866.247909 & -188.554567 & -0.015579 & -9.7759782902 \\
\hline & G & -2054.881767 & -1866.301527 & -188.578788 & -0.001452 & -0.91114452 \\
\hline \multirow{3}{*}{$\mathrm{Cu}$} & $\mathrm{H}$ & 2001605410 & ] 1893112783 & 180558112 & 0014554 & (01327805407 \\
\hline & U & -2081.685449 & -1893.112782 & -188.558113 & -0.014554 & -9.1327805402 \\
\hline & $\mathrm{U}+$ therm & -2081.668451 & -1893.10008 & -188.555511 & -0.01286 & -8.0697786002 \\
\hline \multirow[t]{2}{*}{1} & $\mathrm{H}$ & -2081.667507 & -1893.099135 & -188.554567 & -0.013805 & -8.66277555 \\
\hline & G & -2081.735474 & -1893.153526 & -188.578788 & -0.00316 & -1.9829316001 \\
\hline \multirow[t]{2}{*}{$\mathrm{Zn}$} & $\mathrm{U}$ & -1950.921055 & -1762.353418 & -188.558113 & -0.009524 & -5.97640524 \\
\hline & $\mathrm{U}+$ therm & -1950.904059 & -1762.34051 & -188.555511 & -0.008038 & -5.04392538 \\
\hline \multirow[t]{2}{*}{2} & $\mathrm{H}$ & -1950.903115 & -1762.339565 & -188.554567 & -0.008983 & -5.6369223301 \\
\hline & G & -1950.970519 & -1762.395182 & -188.578788 & 0.003451 & 2.16553701 \\
\hline
\end{tabular}


(ii)Table S20: Linear Binding Mode(PBE-PBE)

\begin{tabular}{|c|c|c|c|c|c|c|}
\hline Dopant & Parameters & Cluster+CO2 & doped cluster & $\mathrm{CO} 2$ & Delta(a.u.) & in $\mathrm{kcal} / \mathrm{mol}$ \\
\hline \multirow[t]{2}{*}{$\mathrm{Sc}$} & $\mathrm{U}$ & no binding & -1742.591663 & -188.43982 & & \\
\hline & $\mathrm{U}+$ therm & found & -1742.579103 & -188.437166 & & \\
\hline \multirow[t]{2}{*}{3} & $\mathrm{H}$ & & -1742.578159 & -188.436222 & & \\
\hline & $\mathrm{G}$ & & -1742.632508 & -188.460545 & & \\
\hline \multirow{2}{*}{$\mathrm{Ti}$} & $\mathrm{II}$ & & -1754177509 & -18843982 & & \\
\hline & $\mathrm{U}+$ therm & no binding & -1754.165301 & -188.437166 & & \\
\hline \multirow[t]{2}{*}{4} & $\mathrm{H}$ & found & -1754.164356 & -188.436222 & & \\
\hline & $\mathrm{G}$ & & -1754.218372 & -188.460545 & & \\
\hline \multirow[t]{2}{*}{$\mathrm{V}$} & $\mathrm{U}$ & -1955.92573 & -1767.470132 & -188.43982 & -0.015778 & -9.90085278 \\
\hline & U+therm & -1955.9089 & -1767.457565 & -188.437166 & -0.014169 & -8.89118919 \\
\hline \multirow[t]{2}{*}{5} & $\mathrm{H}$ & -1955.907956 & -1767.456621 & -188.436222 & -0.015113 & -9.48355863 \\
\hline & G & -1955.975719 & -1767.511215 & -188.460545 & -0.003959 & -2.4843120901 \\
\hline \multirow[t]{2}{*}{$\mathrm{Cr}$} & $\mathrm{U}$ & -1970.919324 & -1782.466283 & -188.43982 & -0.013221 & -8.29630971 \\
\hline & U+therm & -1970.902385 & -1782.453584 & -188.437166 & -0.011635 & -7.30107885 \\
\hline \multirow[t]{2}{*}{6} & $\mathrm{H}$ & -1970.901441 & -1782.452639 & -188.436222 & -0.01258 & -7.8940757999 \\
\hline & $G$ & -1970.968742 & -1782.507975 & -188.460545 & -0.000222 & -0.1393072201 \\
\hline \multirow{2}{*}{$\mathrm{Mn}$} & $\mathrm{U}$ & -1988.519295 & -1800.052003 & -188.43982 & -0.027472 & -17.23895472 \\
\hline & U+therm & -1988.503242 & -1800.038708 & -188.437166 & -0.027368 & -17.17369368 \\
\hline \multirow[t]{2}{*}{5} & $\mathrm{H}$ & -1988.502298 & -1800.037764 & -188.436222 & -0.028312 & -17.7660631201 \\
\hline & $G$ & -1988.564494 & -1800.09524 & -188.460545 & -0.008709 & -5.46498459 \\
\hline \multirow[t]{2}{*}{$\mathrm{Fe}$} & $\mathrm{U}$ & -2008.059988 & $\begin{array}{l}-1819.606631 \\
\end{array}$ & -188.43982 & -0.013537 & -8.4946028699 \\
\hline & U+therm & -2008.043041 & -1819.594144 & -188.437166 & -0.011731 & -7.36131981 \\
\hline \multirow[t]{2}{*}{4} & $\mathrm{H}$ & -2008.042097 & -1819.5932 & -188.436222 & -0.012675 & -7.95368925 \\
\hline & $\mathrm{G}$ & -2008.110483 & -1819.647302 & -188.460545 & -0.002636 & -1.65411636 \\
\hline \multirow{2}{*}{$\mathrm{Co}$} & $\overline{I I T}$ & 1902073070 & 1841265590 & 18813087 & 0016655 & -104511790499 \\
\hline & $\mathrm{U}+$ therm & -2029.706322 & -1841.253705 & -188.437166 & -0.015451 & -9.69565701 \\
\hline \multirow[t]{2}{*}{3} & $\mathrm{H}$ & -2029.705378 & -1841.252761 & -188.436222 & -0.016395 & -10.2880264501 \\
\hline & $G$ & -2029.767995 & -1841.305379 & -188.460545 & -0.002071 & -1.2995732101 \\
\hline \multirow{2}{*}{$\mathrm{Ni}$} & $\mathrm{U}$ & -2053.972238 & -1865.523799 & -188.43982 & -0.008619 & -5.4085086898 \\
\hline & $\mathrm{U}+$ therm & -2053.956287 & -1865.511391 & -188.437166 & -0.00773 & -4.8506522999 \\
\hline \multirow[t]{2}{*}{2} & $\mathrm{H}$ & -2053.955343 & -1865.510447 & -188.436222 & -0.008674 & -5.4430217401 \\
\hline & $G$ & -2054.019399 & -1865.564006 & -188.460545 & 0.005152 & 3.2329315201 \\
\hline \multirow[t]{2}{*}{$\mathrm{Cu}$} & $\mathrm{U}$ & -2080.757858 & -1892.307375 & -188.43982 & -0.010663 & -6.69113913 \\
\hline & U+therm & -2080.740539 & -1892.294349 & -188.437166 & -0.009024 & -5.66265024 \\
\hline \multirow[t]{2}{*}{1} & $\mathrm{H}$ & -2080.739595 & -1892.293405 & -188.436222 & -0.009968 & -6.2550196802 \\
\hline & $G$ & -2080.807763 & -1892.348839 & -188.460545 & 0.001621 & 1.0171937102 \\
\hline \multirow[t]{2}{*}{$\mathrm{Zn}$} & $\mathrm{U}$ & -1950.50513 & -1762.058491 & -188.43982 & -0.006819 & -4.27899069 \\
\hline & U+therm & -1950.487406 & -1762.045242 & -188.437166 & -0.004998 & -3.1362949801 \\
\hline \multirow[t]{2}{*}{2} & $\mathrm{H}$ & -1950.486462 & -1762.044298 & -188.436222 & -0.005942 & -3.72866442 \\
\hline & $G$ & -1950.557324 & -1762.101188 & -188.460545 & 0.004409 & 2.7666915899 \\
\hline
\end{tabular}


(iii)Table S21:Parallel Binding Mode(M06-2X)

\begin{tabular}{|c|c|c|c|c|c|c|}
\hline Dopant & Parameters & Cluster+CO2 & doped cluster & $\mathrm{CO} 2$ & Delta(a.u.) & in $\mathrm{kcal} / \mathrm{mol}$ \\
\hline \multirow[t]{2}{*}{ Sc } & $\mathrm{U}$ & -1931.927046 & -1743.294026 & -188.558113 & -0.074907 & -47.00489157 \\
\hline & U+therm & -1931.912828 & -1743.282713 & -188.555511 & -0.074604 & -46.81475604 \\
\hline \multirow[t]{2}{*}{3} & $\mathrm{H}$ & -1931.911884 & -1743.281768 & -188.554567 & -0.075549 & -47.40775299 \\
\hline & $G$ & -1931.969402 & -1743.333214 & -188.578788 & -0.0574 & -36.019074 \\
\hline & & & & & & \\
\hline \multirow[t]{2}{*}{$\mathrm{Ti}$} & $\mathrm{U}$ & -1943.524244 & -1754.902896 & -188.558113 & -0.063235 & -39.68059485 \\
\hline & U+therm & -1943.509315 & -1754.890712 & -188.555511 & -0.063092 & -39.59086092 \\
\hline \multirow[t]{2}{*}{4} & $\mathrm{H}$ & -1943.508371 & -1754.889767 & -188.554567 & -0.064037 & -40.18385787 \\
\hline & $G$ & -1943.567913 & -1754.943157 & -188.578788 & -0.045968 & -28.84537968 \\
\hline \multirow[t]{2}{*}{$\mathrm{V}$} & $\mathrm{U}$ & -1956.783035 & -1768.173594 & -188.558113 & -0.051328 & -32.20883328 \\
\hline & U+therm & -1956.768526 & -1768.161261 & -188.555511 & -0.051754 & -32.47615254 \\
\hline \multirow[t]{2}{*}{5} & $\mathrm{H}$ & -1956.767582 & -1768.160317 & -188.554567 & -0.052698 & -33.06852198 \\
\hline & $\mathrm{G}$ & -1956.826452 & -1768.214447 & -188.578788 & -0.033217 & -20.84399967 \\
\hline \multirow{2}{*}{$\mathrm{Cr}$} & $\mathrm{U}$ & -1971.796832 & -1783.204125 & -188.558113 & -0.034594 & -21.70808094 \\
\hline & $\mathrm{U}+$ therm & -1971.78135 & -1783.191625 & -188.555511 & -0.034214 & -21.46962714 \\
\hline \multirow[t]{2}{*}{6} & $\mathrm{H}$ & -1971.780406 & -1783.190681 & -188.554567 & -0.035158 & -22.06199658 \\
\hline & $G$ & -1971.841907 & -1783.245599 & -188.578788 & -0.01752 & -10.9939752 \\
\hline \multirow[t]{2}{*}{$\mathrm{Mn}$} & $\bar{U}$ & -1989.438873 & -1800.836745 & -188.558113 & -0.044015 & -27.61985265 \\
\hline & U+therm & -1989.423584 & -1800.824192 & -188.555511 & -0.043881 & -27.53576631 \\
\hline \multirow[t]{2}{*}{5} & $\mathrm{H}$ & -1989.42264 & -1800.823248 & -188.554567 & -0.044825 & -28.12813575 \\
\hline & $G$ & -1989.483322 & -1800.878202 & -188.578788 & -0.026332 & -16.52359332 \\
\hline \multirow{2}{*}{$\mathrm{Fe}$} & $\mathrm{U}$ & -2008.931495 & -1820.34396 & -188.558113 & -0.029422 & $-18,46259922$ \\
\hline & U+therm & -2008.916201 & -1820.331669 & -188.555511 & -0.029021 & -18.21096771 \\
\hline \multirow[t]{2}{*}{4} & $\mathrm{H}$ & -2008.915257 & -1820.330725 & -188.554567 & -0.029965 & -18.80333715 \\
\hline & $G$ & -2008.975626 & -1820.384534 & -188.578788 & -0.012304 & -7.7208830399 \\
\hline & & & & & & \\
\hline \multirow[t]{2}{*}{$\mathrm{Co}$} & $\mathrm{U}$ & -2030.589145 & -1842.002932 & -188.558113 & -0.0281 & -17.633031 \\
\hline & U+therm & -2030.574242 & -1841.990523 & -188.555511 & -0.028208 & -17.70080208 \\
\hline \multirow[t]{2}{*}{3} & $\mathrm{H}$ & -2030.573298 & -1841.989579 & -188.554567 & -0.029152 & -18.29317152 \\
\hline & $\mathrm{G}$ & -2030.632847 & -1842.043565 & -188.578788 & -0.010494 & -6.5850899401 \\
\hline \multirow[t]{2}{*}{$\mathrm{Ni}$} & $\mathrm{U}$ & -2054.849722 & -1866.26126 & -188.558113 & -0.030349 & -19.04430099 \\
\hline & U+therm & -2054.83426 & -1866.248854 & -188.555511 & -0.029895 & -18.75941145 \\
\hline \multirow[t]{2}{*}{2} & $\mathrm{H}$ & -2054.833316 & -1866.247909 & -188.554567 & -0.03084 & -19.3524084 \\
\hline & $G$ & -2054.89387 & -1866.301527 & -188.578788 & -0.013555 & -8.50589805 \\
\hline \multirow{2}{*}{$\mathrm{Cu}$} & $\bar{U}$ & -2081730001 & -1893152274 & -188558113 & -0019614 & -1230798114 \\
\hline & $\mathrm{U}+$ therm & -2081.714308 & -1893.139756 & -188.555511 & -0.019041 & -11.94841791 \\
\hline \multirow[t]{2}{*}{1} & $\mathrm{H}$ & -2081.713363 & -1893.138812 & -188.554567 & -0.019984 & -12.54015984 \\
\hline & $G$ & -2081.774088 & -1893.192376 & -188.578788 & -0.002924 & -1.8348392402 \\
\hline \multirow[b]{2}{*}{$\mathrm{Zn}$} & & & & & & \\
\hline & $\frac{\mathrm{U}}{\mathrm{U}+\text { therm }}$ & $\frac{-1950.932607}{-1950.916792}$ & \begin{tabular}{r|}
-1762.353418 \\
-1762.34051
\end{tabular} & $\frac{-188.558113}{-188.555511}$ & $\frac{-0.021076}{-0.020771}$ & $\frac{-13.22540076}{-13.03401021}$ \\
\hline \multirow[t]{2}{*}{2} & $\mathrm{H}$ & -1950.915848 & -1762.339565 & -188.554567 & -0.021716 & -13.62700716 \\
\hline & $G$ & -1950.977573 & -1762.395182 & -188.578788 & -0.003603 & -2.2609185299 \\
\hline
\end{tabular}


(iv)Table S22:Parallel Binding Mode(PBE-PBE)

\begin{tabular}{|c|c|c|c|c|c|c|}
\hline Dopant & Parameters & Cluster+CO2 & doped cluster & $\mathrm{CO} 2$ & Delta(a.u.) & in $\mathrm{kcal} / \mathrm{mol}$ \\
\hline \multirow[t]{2}{*}{$\mathrm{Sc}$} & $\mathrm{U}$ & -1931.10286 & -1742.591663 & -188.43982 & -0.071377 & -44.78978127 \\
\hline & U+therm & -1931.087619 & -1742.579103 & -188.437166 & -0.07135 & -44.7728385 \\
\hline \multirow[t]{2}{*}{3} & $\mathrm{H}$ & -1931.086675 & -1742.578159 & -188.436222 & -0.072294 & -45.36520794 \\
\hline & G & -1931.146401 & -1742.632508 & -188.460545 & -0.053348 & -33.47640348 \\
\hline & & & & & & \\
\hline \multirow[t]{2}{*}{$\mathrm{Ti}$} & $\mathrm{U}$ & -1942.708389 & -1754.212793 & -188.43982 & -0.055776 & -34.99999776 \\
\hline & $\mathrm{U}+$ therm & -1942.69295 & -1754.200387 & -188.437166 & -0.055397 & -34.76217147 \\
\hline \multirow[t]{2}{*}{4} & $\mathrm{H}$ & -1942.692006 & -1754.199443 & -188.436222 & -0.056341 & -35.35454091 \\
\hline & $\mathrm{G}$ & -1942.752618 & -1754.253221 & -188.460545 & -0.038852 & -24.38001852 \\
\hline \multirow[t]{2}{*}{$\mathrm{V}$} & $\mathrm{U}$ & -1955.963402 & -1767.470132 & -188.43982 & -0.05345 & -33.5404095 \\
\hline & U+therm & -1955.947989 & -1767.457565 & -188.437166 & -0.053258 & -33.41992758 \\
\hline \multirow[t]{2}{*}{5} & $\mathrm{H}$ & -1955.947045 & -1767.456621 & -188.436222 & -0.054202 & -34.01229702 \\
\hline & $\mathrm{G}$ & -1956.007872 & -1767.511215 & -188.460545 & -0.036112 & -22.66064112 \\
\hline \multirow[t]{2}{*}{$\mathrm{Cr}$} & $\mathrm{U}$ & -1970.940755 & -1782.466283 & -188.43982 & -0.034652 & -21.74447652 \\
\hline & U+therm & -1970.925032 & -1782.453584 & -188.437166 & -0.034282 & -21.51229782 \\
\hline \multirow[t]{2}{*}{6} & $\mathrm{H}$ & -1970.924088 & -1782.452639 & -188.436222 & -0.035227 & -22.10529477 \\
\hline & $\mathrm{G}$ & -1970.986127 & -1782.507975 & -188.460545 & -0.017607 & -11.04856857 \\
\hline \multirow[t]{2}{*}{ Mn } & $\mathrm{U}$ & -1988.553922 & -1800.07833 & -188.43982 & -0.035772 & -22.44728772 \\
\hline & U+therm & -1988.538061 & -1800.065703 & -188.437166 & -0.035192 & -22.08333192 \\
\hline \multirow[t]{2}{*}{5} & $\mathrm{H}$ & -1988.537117 & -1800.064759 & -188.436222 & -0.036136 & -22.67570136 \\
\hline & $\mathrm{G}$ & -1988.599292 & -1800.11962 & -188.460545 & -0.019127 & -12.00238377 \\
\hline \multirow[t]{2}{*}{$\mathrm{Fe}$} & $\mathrm{U}$ & -2008.079413 & -1819.606631 & -188.43982 & -0.032962 & -20.68398462 \\
\hline & U+therm & -2008.064321 & -1819.594144 & -188.437166 & -0.033011 & -20.71473261 \\
\hline \multirow[t]{2}{*}{4} & $\mathrm{H}$ & -2008.063376 & -1819.5932 & -188.436222 & -0.033954 & -21.30647454 \\
\hline & $\mathrm{G}$ & -2008.123459 & -1819.647302 & -188.460545 & -0.015612 & -9.7966861199 \\
\hline \multirow{2}{*}{$\mathrm{Co}$} & $\bar{U}$ & -2029734965 & -1841265599 & $\begin{array}{l}-18843982 \\
\end{array}$ & -0029546 & -1854041046 \\
\hline & U+therm & -2029.719148 & -1841.253705 & -188.437166 & -0.028277 & -17.74410027 \\
\hline \multirow[t]{2}{*}{3} & $\mathrm{H}$ & -2029.718204 & -1841.252761 & -188.436222 & -0.029221 & -18.33646971 \\
\hline & $G$ & -2029.780178 & -1841.305379 & -188.460545 & -0.014254 & -8.9445275401 \\
\hline \multirow[t]{2}{*}{$\mathrm{Ni}$} & $\mathrm{U}$ & -2053.989672 & -1865.523799 & -188.43982 & -0.026053 & -16.34851803 \\
\hline & U+therm & -2053.974015 & -1865.511391 & -188.437166 & -0.025458 & -15.97514958 \\
\hline \multirow[t]{2}{*}{2} & $\mathrm{H}$ & -2053.973071 & -1865.510447 & -188.436222 & -0.026402 & -16.56751902 \\
\hline & $\mathrm{G}$ & -2054.033945 & -1865.564006 & -188.460545 & -0.009394 & -5.8948289401 \\
\hline \multirow[t]{2}{*}{$\mathrm{Cu}$} & $\mathrm{U}$ & -2080.798671 & -1892.337699 & -188.43982 & -0.021152 & -13.27309152 \\
\hline & U+therm & -2080.782587 & -1892.324938 & -188.437166 & -0.020483 & -12.85328733 \\
\hline \multirow[t]{2}{*}{1} & $\mathrm{H}$ & -2080.781643 & -1892.323994 & -188.436222 & -0.021427 & -13.44565677 \\
\hline & $\mathrm{G}$ & -2080.843506 & -1892.378052 & -188.460545 & -0.004909 & -3.0804465901 \\
\hline \multirow[t]{2}{*}{$\mathrm{Zn}$} & $\mathrm{U}$ & -1950.519207 & -1762.058491 & -188.43982 & -0.020896 & -13.11244896 \\
\hline & $\mathrm{U}+$ therm & -1950.50304 & -1762.045242 & -188.437166 & -0.020632 & -12.94678632 \\
\hline \multirow[t]{2}{*}{2} & $\mathrm{H}$ & -1950.502096 & -1762.044298 & -188.436222 & -0.021576 & -13.53915576 \\
\hline & $G$ & -1950.565071 & -1762.101188 & -188.460545 & -0.003338 & -2.09462838 \\
\hline
\end{tabular}




\section{Cartesian Coordinates and Normal Modes of Vibrations of Pristine clusters $\underline{\mathrm{Al}} 6 \underline{6}$ Cluster \\ M06-2X/6-311g(d,p) [g]}

\begin{tabular}{|c|c|c|c|c|c|c|c|}
\hline & \\
\hline & & \multirow{2}{*}{$\begin{array}{c}\text { Center } \\
\text { Number }\end{array}$} & \multirow{2}{*}{$\begin{array}{l}\text { Atomic } \\
\text { Number }\end{array}$} & \multirow{2}{*}{$\begin{array}{c}\text { Atomic } \\
\text { Type }\end{array}$} & \multicolumn{2}{|c|}{ Coordinates (Angstroms) } & \\
\hline & & & & & X $\quad Y$ & $\begin{array}{l}\mathrm{Z} \\
\mathrm{H}\end{array}$ & \\
\hline & & 1 & 13 & 1.188831 & 1.200679 & -0.968058 & \\
\hline & & 2 & 13 & -0.445484 & 1.628009 & 0.971335 & \\
\hline & & 3 & 13 & 1.632648 & -0.430223 & 0.970470 & \\
\hline & & 4 & 13 & 0.445485 & -1.628010 & -0.971334 & \\
\hline & & 5 & 13 & -1.188831 & -1.200679 & 0.968058 & \\
\hline & & 6 & 13 & -1.632649 & 0.430223 & -0.970470 & \\
\hline \multirow[t]{10}{*}{ Frequencies -- } & 96.6656 & & .8352 & 139.1659 & 195.5097 & 195.6305 & 213.6841 \\
\hline & & \multicolumn{5}{|c|}{ PBE-PBE/6-311g(d,p)[g] } & \\
\hline & & \multirow{2}{*}{$\begin{array}{l}\text { Center } \\
\text { Number }\end{array}$} & \multirow{2}{*}{$\begin{array}{l}\text { Atomic } \\
\text { Number }\end{array}$} & Atomic & \multicolumn{2}{|c|}{ Coordinates (Angstroms) } & \\
\hline & & & & Type & X $\quad Y$ & $\begin{array}{l}\mathrm{Z} \\
\mathrm{Z}\end{array}$ & \\
\hline & & 1 & 13 & 3.170444 & -0.300516 & 1.525728 & \\
\hline & & 2 & 13 & 0.882257 & 0.879294 & 1.415419 & \\
\hline & & 3 & 13 & 1.481074 & -1.781243 & 0.263240 & \\
\hline & & 4 & 13 & 3.109992 & -0.690340 & -1.408976 & \\
\hline & & 5 & 13 & 0.821970 & 0.489813 & -1.519145 & \\
\hline & & 6 & 13 & 2.511304 & 1.970448 & -0.256532 & \\
\hline Frequencies -- & 90.9853 & & .7331 & 120.7784 & 193.4575 & 194.0617 & 214.7063 \\
\hline
\end{tabular}

\begin{tabular}{|c|c|c|c|c|c|c|c|}
\hline & & & M06- & $\frac{\mathrm{Al}_{8}}{\mathrm{X} / 6-311 \mathrm{~g}}$ & $\frac{\Gamma}{d, p)[g]}$ & & \\
\hline & Center & & Atomic & Atomic & Coordinat & tes (Angstroms) & \\
\hline & Number & & Number & Type & X $\quad \mathrm{Y}$ & $\begin{array}{ll}\mathrm{Z} \\
\mathrm{Z}\end{array}$ & \\
\hline & 1 & 13 & 0 & -1.151923 & 1.579283 & 0.537897 & \\
\hline & 2 & 13 & 0 & 1.151927 & 1.579290 & -0.537909 & \\
\hline & 3 & 13 & 0 & -3.041371 & 0.000000 & -0.129288 & \\
\hline & 4 & 13 & 0 & -1.151923 & -1.579283 & 0.537897 & \\
\hline & 5 & 13 & 0 & 1.151927 & -1.579290 & -0.537909 & \\
\hline & 6 & 13 & 0 & -0.526931 & 0.000000 & -1.711868 & \\
\hline & 7 & 13 & 0 & 3.041350 & 0.000000 & 0.129325 & \\
\hline & 8 & 13 & 0 & 0.526944 & 0.000000 & 1.711855 & \\
\hline Frequencies -- 51.2356 & & & & 102.0156 & 105.6615 & 133.8980 & 174.4148 \\
\hline
\end{tabular}




\section{PBE-PBE/6-311g(d,p)[g]}

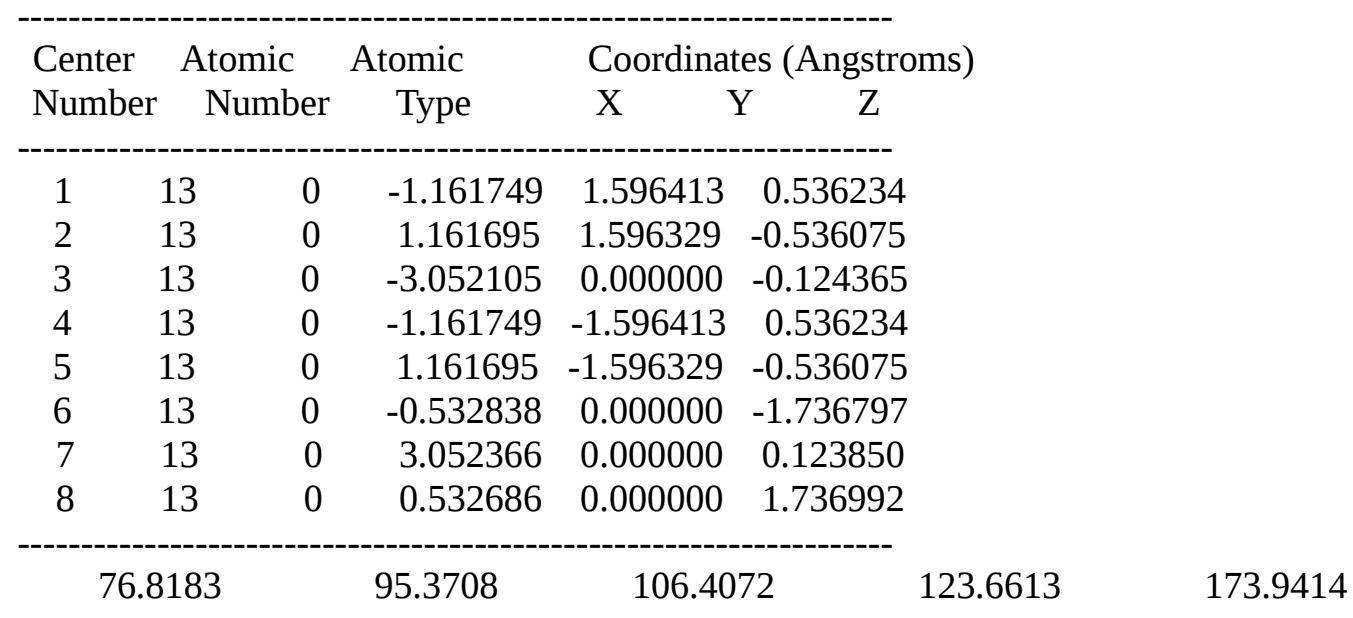

Frequencies -- 43.2280 
Cartesian Coordinates and Normal Modes of Vibrations of Impure Clusters and Complexes

(Only global minima (highlighted by '[g]') and local minima closer in energy are included . Coordinate value for only parallel binding modes are included. Energy values are given in a.u)

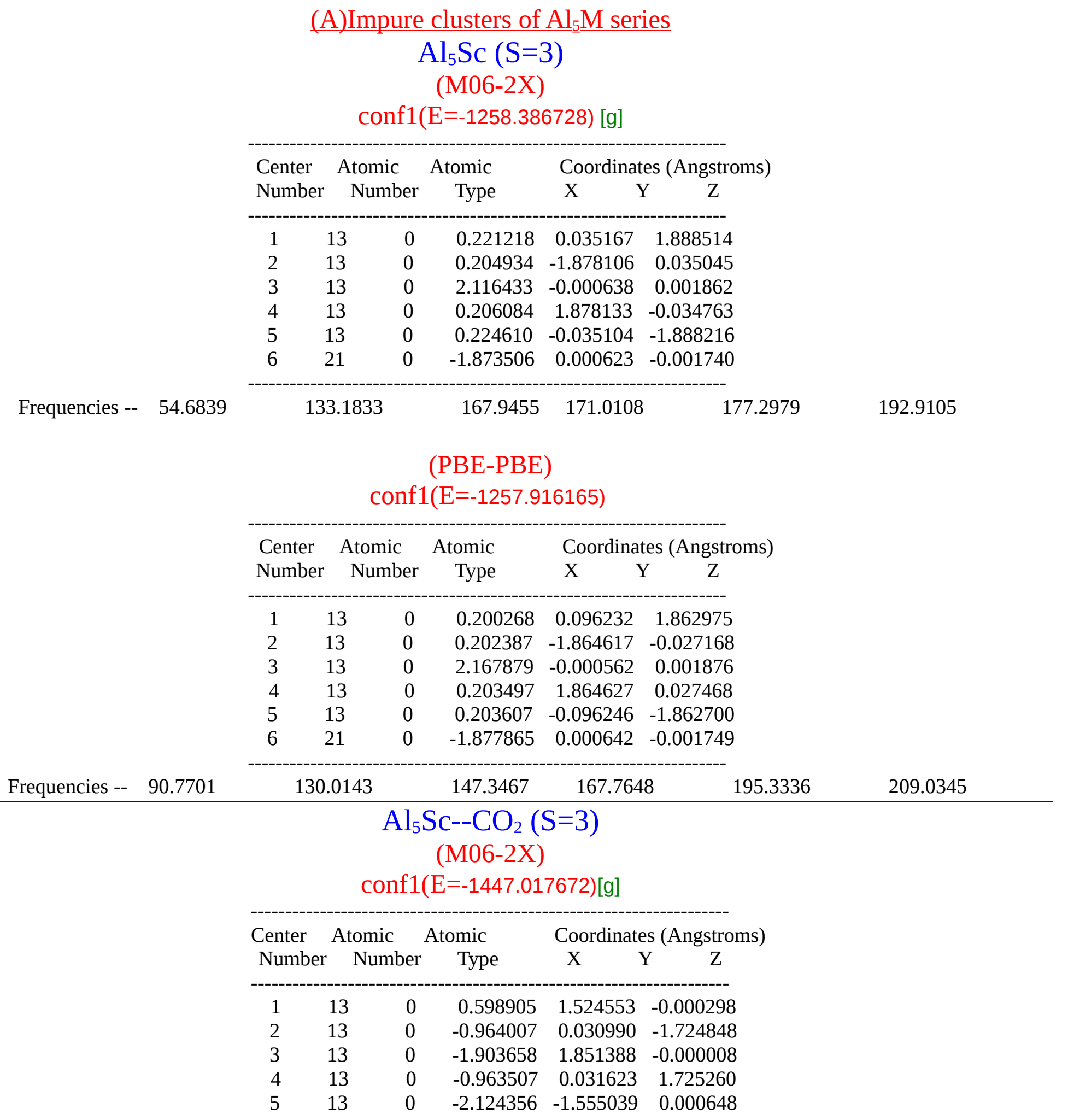




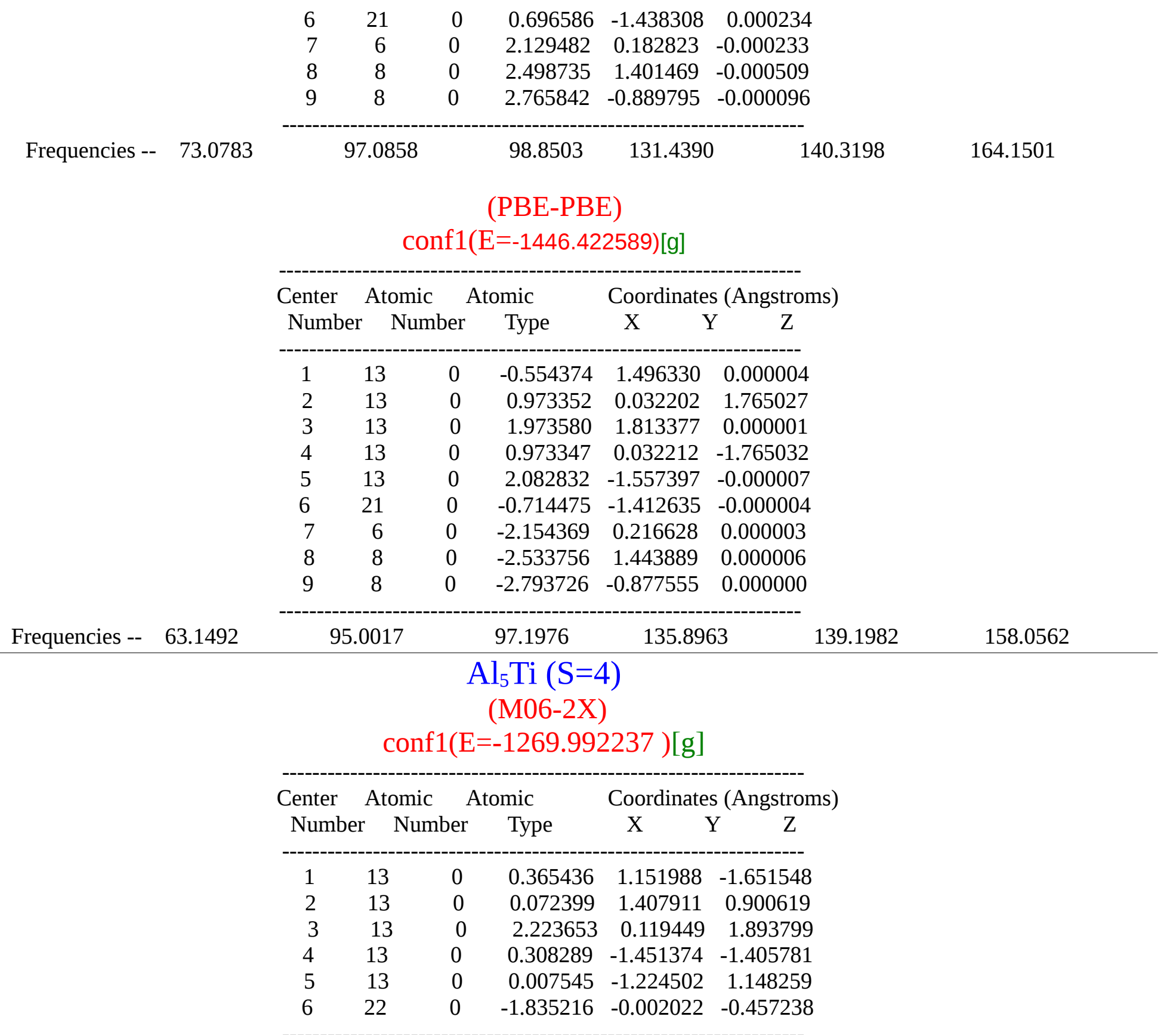

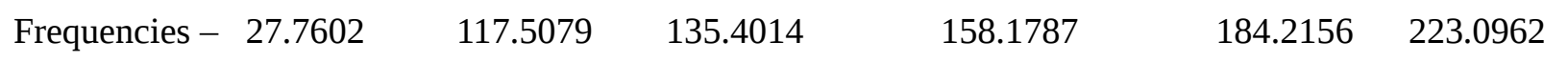

\begin{tabular}{|c|c|c|c|c|}
\hline \multirow{2}{*}{$\begin{array}{l}\text { Center } \\
\text { Number }\end{array}$} & \multirow{2}{*}{$\begin{array}{l}\text { Atomic } \\
\text { I Number }\end{array}$} & \multirow{2}{*}{$\begin{array}{l}\text { Atomic } \\
\text { r Type }\end{array}$} & \multicolumn{2}{|c|}{ Coordinates (Angstroms } \\
\hline & & & $\mathrm{X}$ & $\mathrm{Y} \quad \mathrm{Z}$ \\
\hline 1 & 13 & 0.712565 & 1.226585 & -1.232591 \\
\hline 2 & 13 & -0.187964 & 1.411768 & 1.275117 \\
\hline 3 & 13 & 1.984040 & 0.026060 & 0.791838 \\
\hline
\end{tabular}




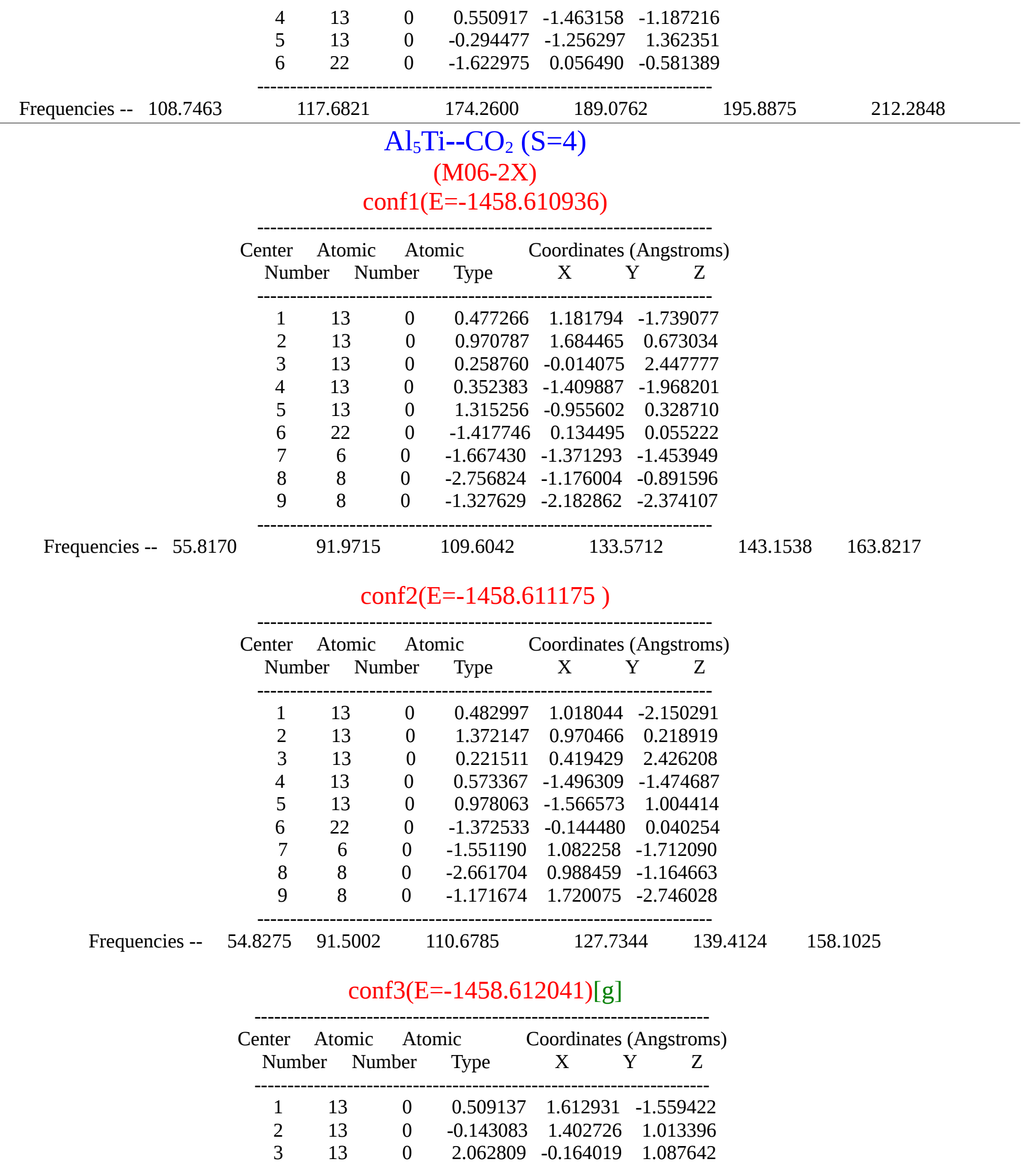




\begin{tabular}{|c|c|c|c|c|c|c|}
\hline & & & & & & \\
\hline & & & & & & \\
\hline & 5 & 13 & 0 & -0.081869 & -1.435717 & 1.281637 \\
\hline & 6 & 22 & 0 & -1.496824 & -0.073290 & -0.863913 \\
\hline & 7 & 6 & 0 & -1.978464 & -1.605878 & 0.465553 \\
\hline & 8 & 8 & 0 & -1.707909 & -2.396869 & 1.427806 \\
\hline & 9 & 8 & 0 & -2.990716 & -1.439263 & -0.234921 \\
\hline Frequencies -- & 57.2021 & 88.6897 & & 99.5476 & 126.594 & 155. \\
\hline & & $\mathrm{CO}$ & $\mathrm{hf} 4($ & $E=-1458.6$ & 511999) & \\
\hline & Center & Atomic & & mic & Coordinates ( & (Angstroms \\
\hline & Numb & er Num & ber & Type & $X \quad Y$ & $\mathrm{Y} \quad \mathrm{Z}$ \\
\hline & 1 & 13 & 0 & 0.974880 & 0.853374 & -1.334999 \\
\hline & 2 & 13 & 0 & 0.127204 & 1.459388 & 1.310966 \\
\hline & 3 & 13 & 0 & 2.243037 & 0.170385 & 0.960727 \\
\hline & 4 & 13 & 0 & 0.421386 & -1.748316 & -1.405073 \\
\hline & 5 & 13 & 0 & 0.059685 & -1.402116 & 1.213095 \\
\hline & 6 & 22 & 0 & -1.506497 & -0.034947 & -0.573047 \\
\hline & 7 & 6 & 0 & -1.843844 & 1.590851 & 0.687455 \\
\hline & 8 & 8 & 0 & -1.473914 & 2.440643 & 1.562545 \\
\hline & 9 & 8 & 0 & -2.921541 & 1.391072 & 0.102644 \\
\hline
\end{tabular}

174.1631

$\begin{array}{lllll}\text { Frequencies -- } 66.0189 & 94.8283 & 104.5530 & 179.0246 & 126.5648\end{array}$

157.9193

(PBE-PBE)

$\operatorname{conf1}(\mathrm{E}=-1458.026367)$

\begin{tabular}{|c|c|c|c|c|c|}
\hline \multirow{2}{*}{$\begin{array}{l}\text { Center } \\
\text { Numbe }\end{array}$} & Atomic & \multicolumn{2}{|c|}{ Atomic } & \multicolumn{2}{|c|}{ Coordinates (Angstroms } \\
\hline & er Numb & per & Type & $\mathrm{X} \quad \mathrm{I}$ & $\mathrm{Y} \quad \mathrm{Z}$ \\
\hline 1 & 13 & 0 & 0.505923 & 1.270414 & -1.791344 \\
\hline 2 & 13 & 0 & 1.058041 & 1.616409 & 0.656493 \\
\hline 3 & 13 & 0 & 0.177441 & 0.078621 & 2.475767 \\
\hline 4 & 13 & 0 & 0.352665 & -1.327430 & -2.005960 \\
\hline 5 & 13 & 0 & 1.229169 & -1.055209 & 0.383380 \\
\hline 6 & 22 & 0 & -1.305555 & 0.114019 & -0.002714 \\
\hline 7 & 6 & 0 & -1.698409 & -1.258337 & -1.533985 \\
\hline 8 & 8 & 0 & -1.414191 & -2.049140 & -2.499481 \\
\hline 9 & 8 & 0 & -2.777857 & -1.012837 & -0.915991 \\
\hline
\end{tabular}

Frequencies --

$\begin{array}{lllll}75.7144 & 92.2324 & 117.5148 & 122.1490 & 138.2733\end{array}$

$\operatorname{conf} 2(E=-1458.031766)$

\begin{tabular}{ccccc} 
Center Atomic Atomic & \multicolumn{2}{c}{ Coordinates (Angstroms) } \\
Number Number Type & X & Y & Z
\end{tabular}




$\begin{array}{rrrrrr}1 & 13 & 0 & 0.361403 & 1.531019 & -1.716775 \\ 2 & 13 & 0 & -0.058771 & 1.399746 & 0.928728 \\ 3 & 13 & 0 & 2.250298 & -0.081917 & 1.118204 \\ 4 & 13 & 0 & 0.976822 & -1.011999 & -1.131749 \\ 5 & 13 & 0 & -0.007695 & -1.300291 & 1.411733 \\ 6 & 22 & 0 & -1.470185 & -0.179772 & -0.633514 \\ 7 & 6 & 0 & -1.957904 & -1.611049 & 0.768142 \\ 8 & 8 & 0 & -1.637981 & -2.338711 & 1.779974 \\ 9 & 8 & 0 & -3.004240 & -1.487634 & 0.073972\end{array}$

$\begin{array}{cccccc}\text { Frequencies -- } 54.1829 & 77.9056 & 94.9909 & 141.0391 & 158.8311 & 163.9900 \\ & \text { conf3(E=-1458.031779) }[\mathrm{g}]\end{array}$

$\begin{array}{cccc}\text { Center Atomic Atomic } & \text { Coordinates (Angstroms) } \\ \text { Number Number Type } & \text { X } & \text { Y Z Z }\end{array}$

$\begin{array}{rrrrrr} & & & & & \\ 1 & 13 & 0 & 0.987424 & 0.776989 & -1.290499 \\ 2 & 13 & 0 & 0.207363 & 1.360153 & 1.275049 \\ 3 & 13 & 0 & 2.441900 & 0.107823 & 0.945372 \\ 4 & 13 & 0 & 0.343573 & -1.815698 & -1.526110 \\ 5 & 13 & 0 & 0.135256 & -1.378678 & 1.111868 \\ 6 & 22 & 0 & -1.405965 & 0.013063 & -0.504002 \\ 7 & 6 & 0 & -1.790747 & 1.596991 & 0.757930 \\ 8 & 8 & 0 & -2.888281 & 1.394473 & 0.169531 \\ 9 & 8 & 0 & -1.397021 & 2.436287 & 1.650388\end{array}$

$\begin{array}{lllllll}\text { Frequencies -- } & 61.1576 & 77.9015 & 98.5008 & 142.7145 & 158.5291 & 163.9731\end{array}$

\begin{tabular}{|c|c|c|c|c|c|}
\hline \multicolumn{6}{|c|}{$\operatorname{conf} 4(E=-1458.026380)$} \\
\hline \multirow{2}{*}{$\begin{array}{l}\text { Center } \\
\text { Numbe }\end{array}$} & Atomic & & Atomic & \multicolumn{2}{|c|}{ Coordinates (Angstroms) } \\
\hline & & Number & Type & X & $\mathrm{Y} \quad \mathrm{Z}$ \\
\hline 1 & 13 & 0 & 0.285923 & 1.104753 & -2.138106 \\
\hline 2 & 13 & 0 & 1.305956 & 1.058868 & 0.208476 \\
\hline 3 & 13 & 0 & 0.317063 & 0.299713 & 2.492145 \\
\hline 4 & 13 & 0 & 0.231578 & $3-1.449374$ & -1.591814 \\
\hline 5 & 13 & 0 & 0.932328 & $8-1.529845$ & 0.841536 \\
\hline 6 & 22 & 0 & -1.339378 & $8 \quad 0.087595$ & 0.135457 \\
\hline 7 & 6 & 0 & -1.724290 & 1.287745 & -1.535633 \\
\hline 8 & 8 & 0 & -2.771937 & 1.224318 & -0.824347 \\
\hline 9 & 8 & 0 & -1.446020 & 1.921379 & -2.612081 \\
\hline
\end{tabular}

$\begin{array}{lllllll}\text { Frequencies -- } & 59.7721 & 76.9216 & 92.3885 & 117.5349 & 123.0754 & 138.6379\end{array}$ 


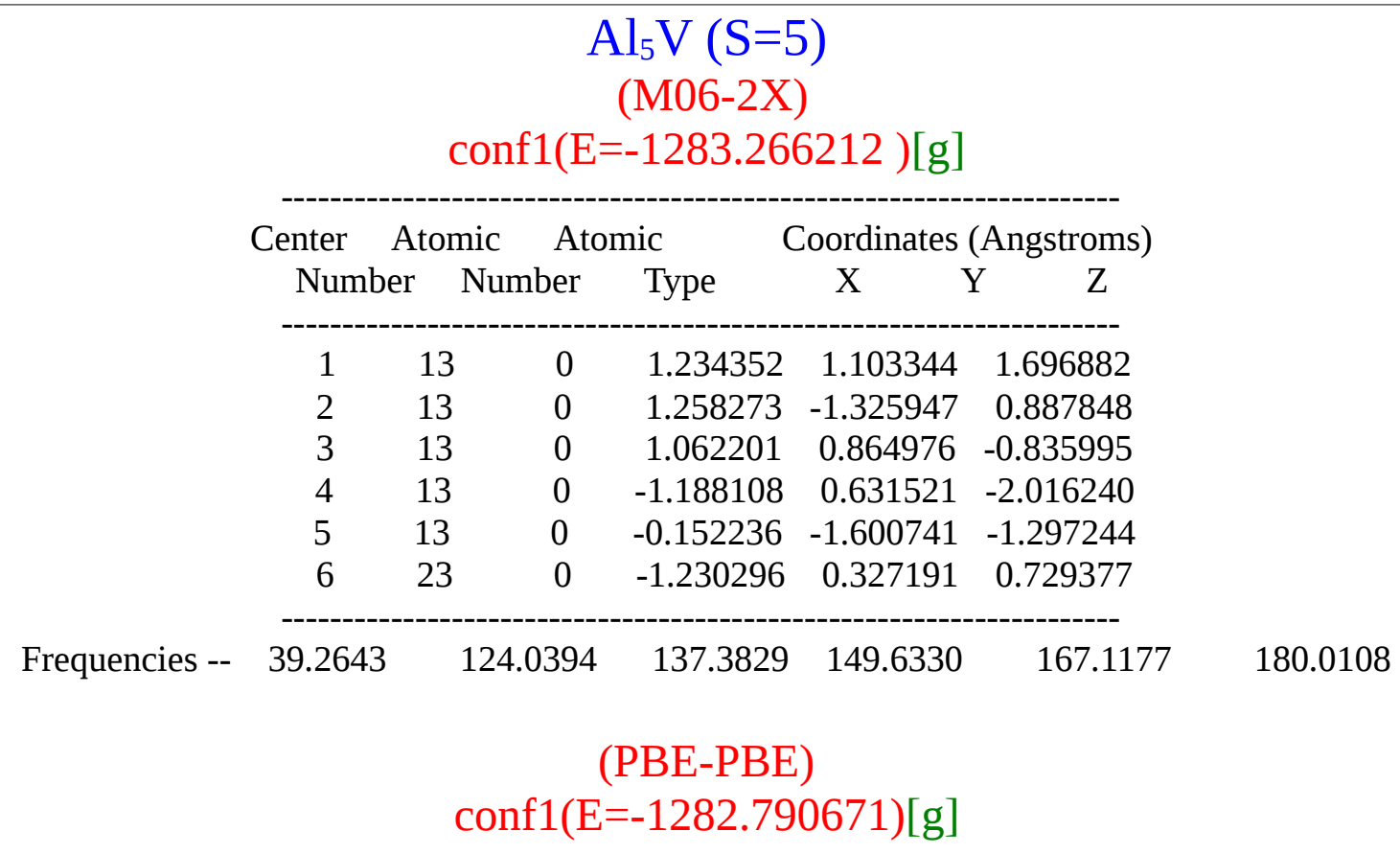

Center Atomic Atomic Coordinates (Angstroms)

\begin{tabular}{rrrrrr} 
Number & Number & Type & \multicolumn{1}{c}{ X } & Y & Z \\
\hline 1 & 13 & 0 & 1.200261 & 1.238706 & 1.738346 \\
2 & 13 & 0 & 1.211903 & -1.281659 & 1.054835 \\
3 & 13 & 0 & 1.098162 & 0.813426 & -0.847568 \\
4 & 13 & 0 & -1.242109 & 0.759940 & -2.032831 \\
5 & 13 & 0 & -0.325381 & -1.583010 & -1.328292 \\
6 & 23 & 0 & -0.958650 & 0.052942 & 0.580138
\end{tabular}

$\begin{array}{lllllll}\text { Frequencies -- } & 34.2847 & 88.2703 & 140.0831 & 145.9094 & 157.9087 & 190.3068\end{array}$

$$
\begin{gathered}
\mathrm{Al}_{5} \mathrm{~V}--\mathrm{CO}_{2}(\mathrm{~S}=5) \\
(\mathrm{M} 06-2 \mathrm{X}) \\
\operatorname{conf1}(\mathrm{E}=-1471.870111)[\mathrm{g}]
\end{gathered}
$$

Center Atomic Atomic Coordinates (Angstroms)

\begin{tabular}{rrrrrr} 
Number & Number & Type & X & $Y$ & Z \\
\hline 1 & 6 & 0 & 2.167368 & 0.581413 & 0.091012 \\
2 & 8 & 0 & 3.064734 & -0.235864 & -0.358387 \\
3 & 8 & 0 & 2.255165 & 1.694000 & 0.605716 \\
4 & 13 & 0 & -0.881151 & -0.801096 & -1.467434 \\
5 & 13 & 0 & -0.219046 & -1.520885 & 1.364054 \\
6 & 13 & 0 & 1.471382 & -1.154855 & -0.680143 \\
7 & 13 & 0 & -2.482222 & 1.022664 & -0.952762 \\
8 & 13 & 0 & -2.536586 & -0.634138 & 0.908666 \\
9 & 23 & 0 & 0.141353 & 1.113653 & 0.461337
\end{tabular}




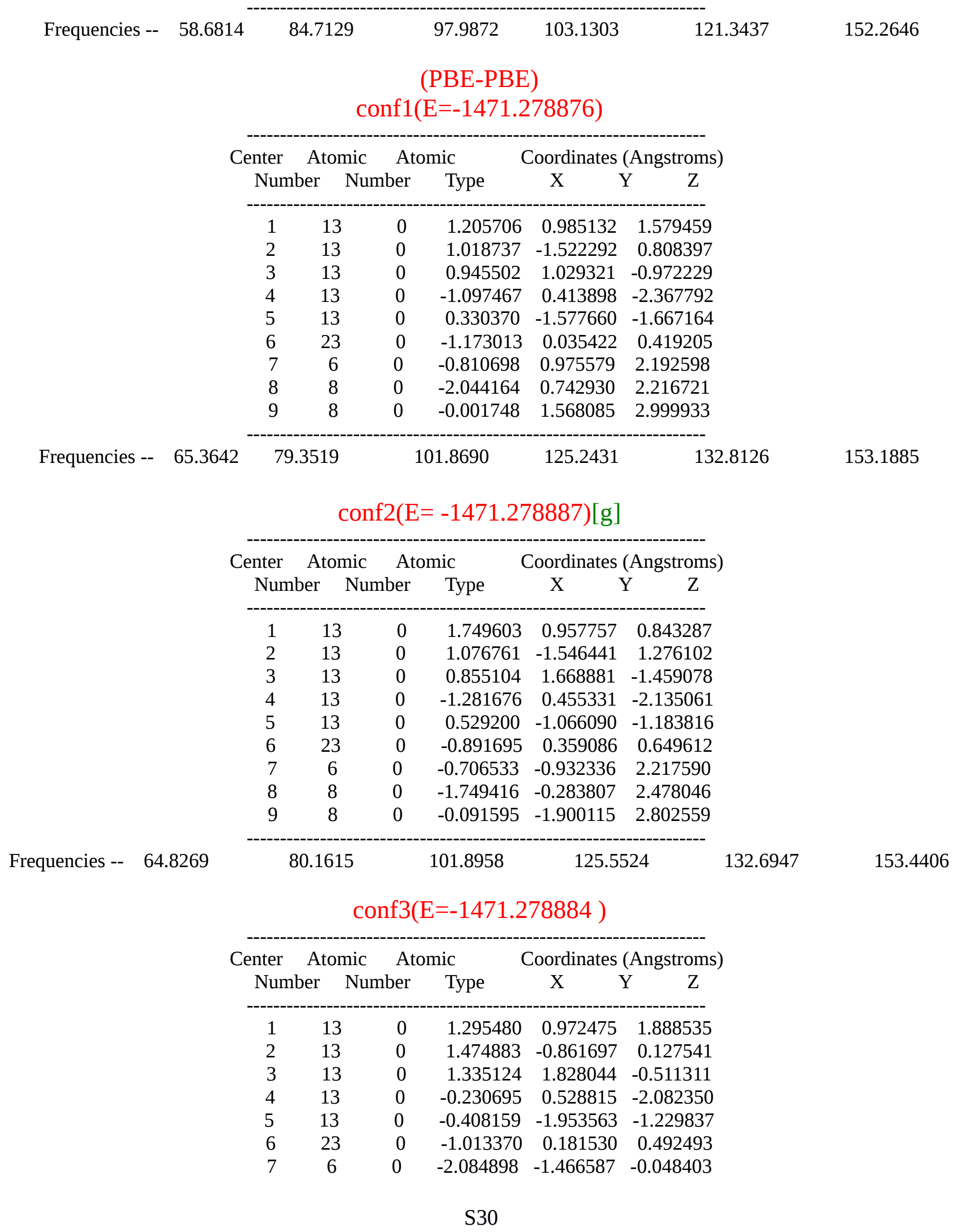




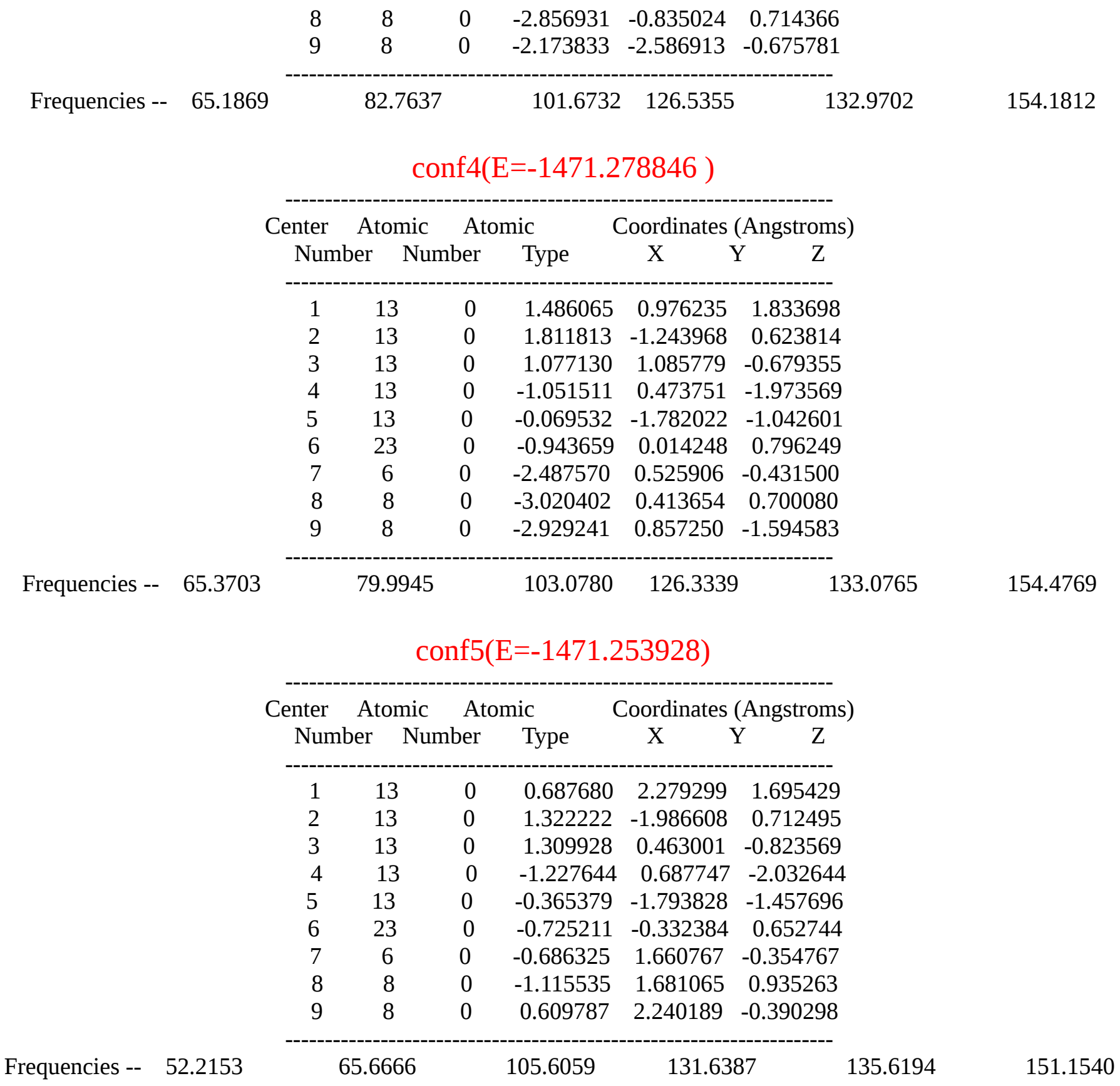

$\mathrm{Al}_{5} \mathrm{Cr}(\mathrm{S}=6)$

(M06-2X)

conf1(E=-1298.310167)[g]

\begin{tabular}{|c|c|c|c|c|}
\hline \multirow{2}{*}{$\begin{array}{l}\text { Center } \\
\text { Number }\end{array}$} & \multirow{2}{*}{$\begin{array}{l}\text { Atomic } \\
\text { Number }\end{array}$} & \multirow{2}{*}{$\begin{array}{l}\text { Atomic } \\
\text { Type }\end{array}$} & \multicolumn{2}{|c|}{ Coordinates (AngstroI } \\
\hline & & & $\mathrm{X}$ & $\mathrm{Y} \quad \mathrm{Z}$ \\
\hline 1 & 13 & & & -1.518924 \\
\hline 2 & 13 & 0.361107 & 2.234806 & 0.876908 \\
\hline 3 & 13 & 1.546176 & -0.000118 & 0.645896 \\
\hline
\end{tabular}




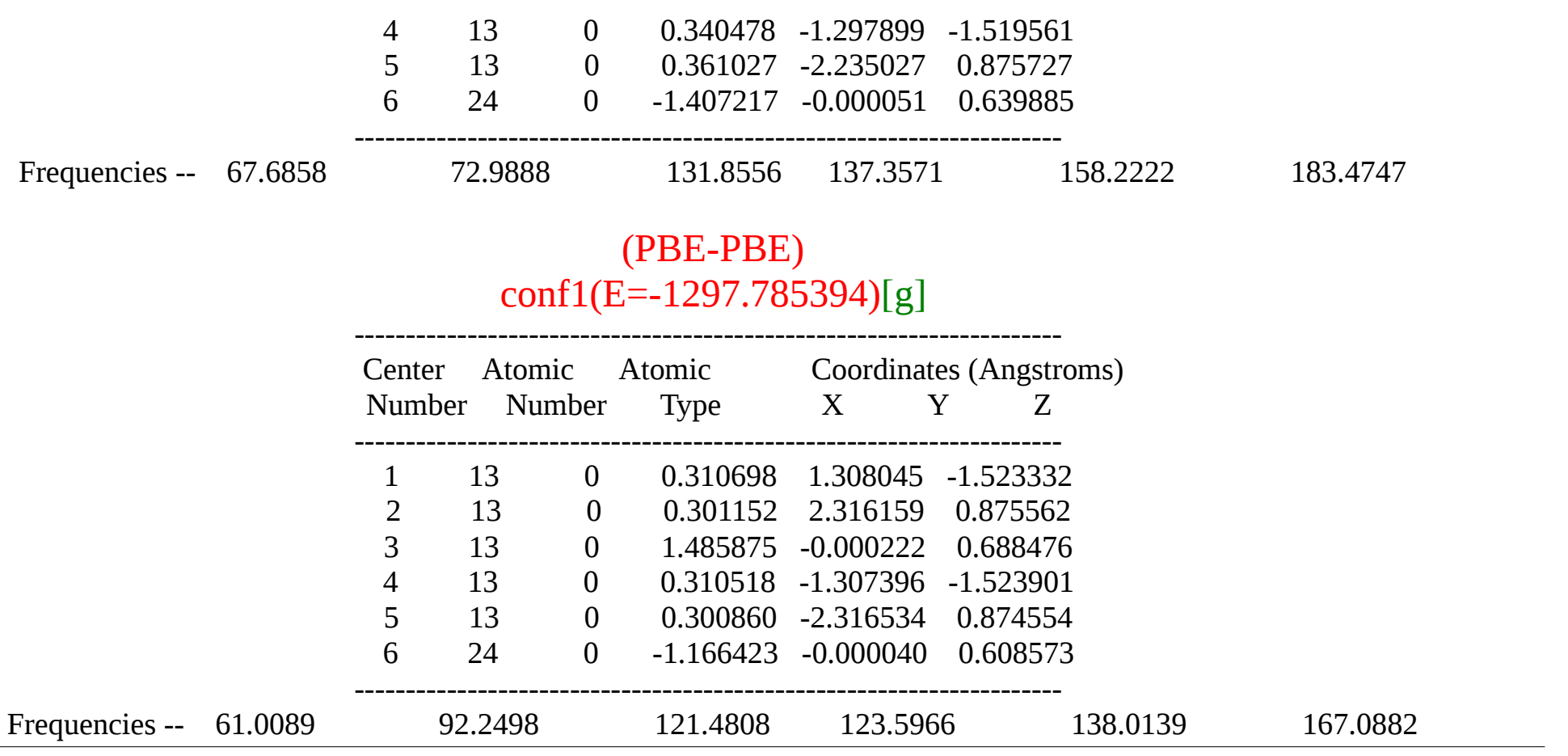

$$
\begin{gathered}
\mathrm{Al}_{5} \mathrm{Cr}--\mathrm{CO}_{2}(\mathrm{~S}=6) \\
(\mathrm{M} 06-2 \mathrm{X}) \\
\operatorname{conf1}(\mathrm{E}=-1486.901604)
\end{gathered}
$$

\begin{tabular}{|c|c|c|c|c|c|}
\hline \multirow{2}{*}{$\begin{array}{l}\text { Center } \\
\text { Number }\end{array}$} & \multirow{2}{*}{\multicolumn{2}{|c|}{$\begin{array}{l}\text { Atomic } \\
\text { Number }\end{array}$}} & \multirow{2}{*}{$\begin{array}{r}\text { Atomic } \\
\text { Type }\end{array}$} & \multicolumn{2}{|c|}{ Coordinates (Angstroms) } \\
\hline & & & & $\mathrm{X} \quad \mathrm{Y}$ & $\begin{array}{l}\mathrm{Y} \\
\mathrm{Z}\end{array}$ \\
\hline 1 & 13 & 0 & -0.123157 & 1.276540 & -1.209388 \\
\hline 2 & 13 & 0 & 0.978142 & 2.298349 & 0.993439 \\
\hline 3 & 13 & 0 & 1.645878 & -0.137327 & 0.813103 \\
\hline 4 & 13 & 0 & 0.369624 & -1.170932 & -1.508633 \\
\hline 5 & 13 & 0 & 0.705551 & -2.490737 & 0.700808 \\
\hline 6 & 24 & 0 & -1.393697 & -0.465961 & 0.821128 \\
\hline 7 & 6 & 0 & -1.368928 & 2.021973 & 0.363130 \\
\hline 8 & 8 & 0 & -2.421975 & 1.517744 & 0.762934 \\
\hline 9 & 8 & 0 & -0.710911 & 2.891433 & 1.146170 \\
\hline & 0.94 & 431 & 99.1342 & 120.4018 & 133.6410 \\
\hline
\end{tabular}

\begin{tabular}{|c|c|c|c|c|}
\hline \multirow{2}{*}{$\begin{array}{l}\text { Center } \\
\text { Number }\end{array}$} & \multirow{2}{*}{$\begin{array}{l}\text { Atomic } \\
\text { Number }\end{array}$} & \multirow{2}{*}{$\begin{array}{r}\text { Atomic } \\
\text { Type }\end{array}$} & \multicolumn{2}{|c|}{ Coordinates (Angstroms } \\
\hline & & & $\mathrm{X}$ & $\mathrm{Y} \quad \mathrm{Z}$ \\
\hline 1 & 13 & 0.388229 & 1.170560 & -1.520629 \\
\hline 2 & 13 & 0.726776 & 2.481804 & 0.694516 \\
\hline 3 & 13 & 1.596752 & 0.101503 & 0.820660 \\
\hline 4 & 13 & -0.178582 & -1.262540 & -1.228003 \\
\hline
\end{tabular}

Frequencies -- 56.5206 


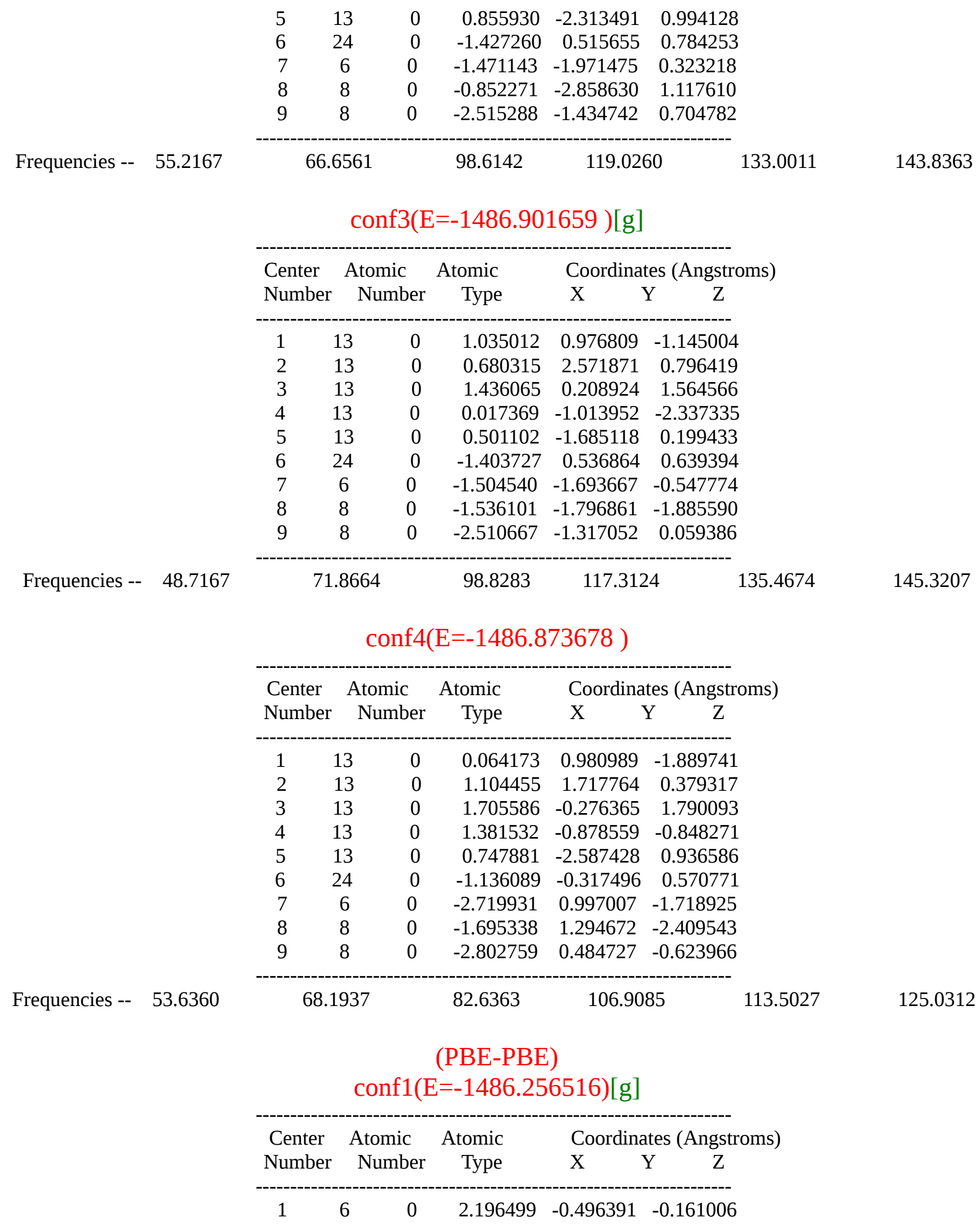




$\begin{array}{rrrrrr}2 & 8 & 0 & 3.028952 & 0.301762 & 0.434565 \\ 3 & 8 & 0 & 2.359213 & -1.560935 & -0.783577 \\ 4 & 13 & 0 & -2.511901 & 0.547642 & -0.896876 \\ 5 & 13 & 0 & -2.230520 & -1.477883 & 0.670101 \\ 6 & 13 & 0 & -1.027760 & 0.581664 & 1.490531 \\ 7 & 13 & 0 & -0.370794 & 1.904857 & -0.988963 \\ 8 & 13 & 0 & 1.354994 & 1.160859 & 0.859700 \\ 9 & 24 & 0 & 0.217089 & -1.077000 & -0.463190\end{array}$

Frequencies -- 49.1230

59.6652

78.0625

106.6085

136.5118

147.6688

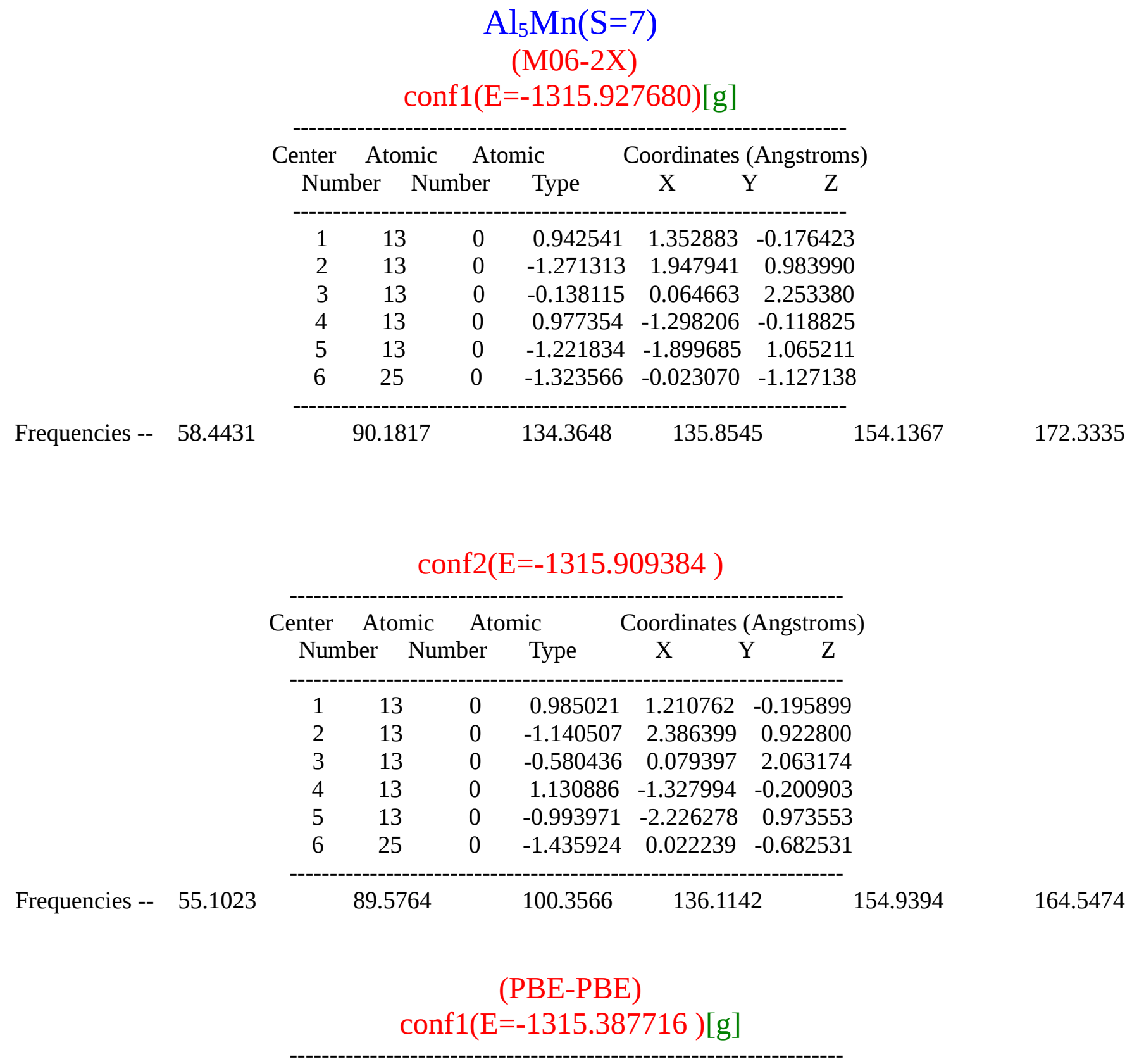




\begin{tabular}{|c|c|c|c|c|c|}
\hline \multirow{2}{*}{$\begin{array}{l}\text { Center } \\
\text { Number }\end{array}$} & \multicolumn{2}{|c|}{ Atomic } & Atomic & \multicolumn{2}{|c|}{ Coordinates (Angstroms } \\
\hline & & Number & Type & $X \quad Y$ & $\mathrm{Y} \quad \mathrm{Z}$ \\
\hline 1 & 13 & 0 & 0.907209 & 1.350076 & -0.215377 \\
\hline 2 & 13 & 0 & -1.304809 & 1.835155 & 0.962016 \\
\hline 3 & 13 & 0 & -0.093833 & 0.068076 & 2.358110 \\
\hline 4 & 13 & 0 & 0.940712 & -1.298897 & -0.161062 \\
\hline 5 & 13 & 0 & -1.256690 & -1.789288 & 1.040629 \\
\hline 6 & 25 & 0 & -1.227520 & -0.020596 & -1.104121 \\
\hline
\end{tabular}

Frequencies -- 51.2044

101.4224
$\mathrm{Al}_{5} \mathrm{Mn}--\mathrm{CO}_{2}(\mathrm{~S}=7)$

(M06-2X)

\section{conf1(E=-1504.527807)[g]}

\begin{tabular}{ccccc} 
Center & Atomic & Atomic & \multicolumn{2}{c}{ Coordinates (Angstroms) } \\
Number & Number & Type & X & Y Z
\end{tabular}

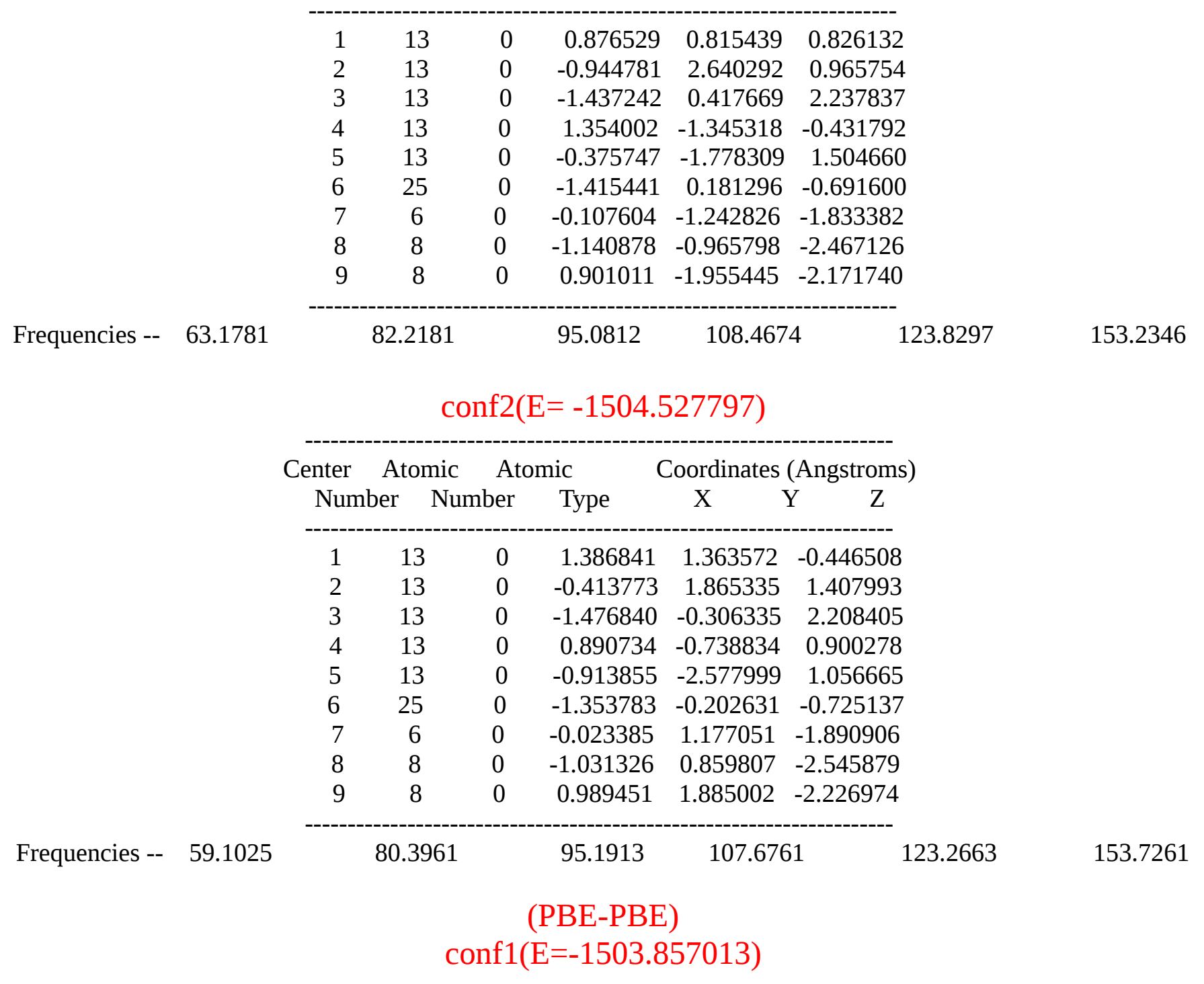




\begin{tabular}{|c|c|c|c|c|c|}
\hline \multirow{2}{*}{$\begin{array}{l}\text { Center } \\
\quad \text { Numb }\end{array}$} & Atomic & \multicolumn{2}{|c|}{ Atomic } & \multicolumn{2}{|c|}{ Coordinates (Angstroms) } \\
\hline & er Numb & & Tyре & X & $\mathrm{Z}$ \\
\hline 1 & 13 & 0 & 0.853467 & 1.892246 & -0.431820 \\
\hline 2 & 13 & 0 & -1.131061 & 1.525899 & 1.219885 \\
\hline 3 & 13 & 0 & 0.107109 & -0.139257 & 2.799269 \\
\hline 4 & 13 & 0 & 1.292824 & -0.644053 & 0.367258 \\
\hline 5 & 13 & 0 & -1.173523 & -1.462627 & 1.080633 \\
\hline 6 & 25 & 0 & -0.623854 & -0.116485 & -1.268321 \\
\hline 7 & 6 & 0 & -1.857723 & -1.762837 & -0.886053 \\
\hline 8 & 8 & 0 & -2.132241 & -1.674001 & -2.094203 \\
\hline 9 & 8 & 0 & -2.397651 & -2.495698 & 0.060101 \\
\hline
\end{tabular}

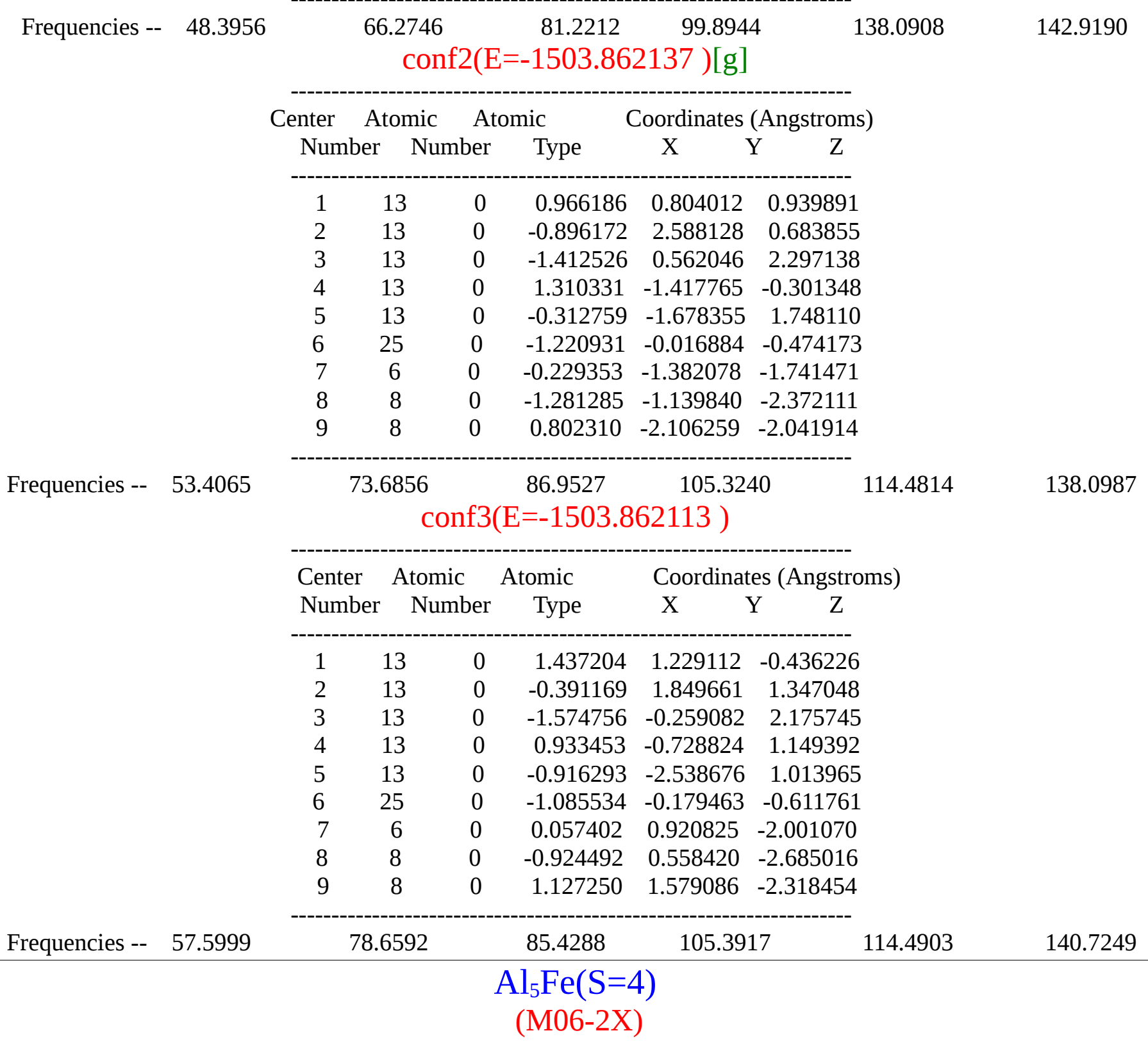




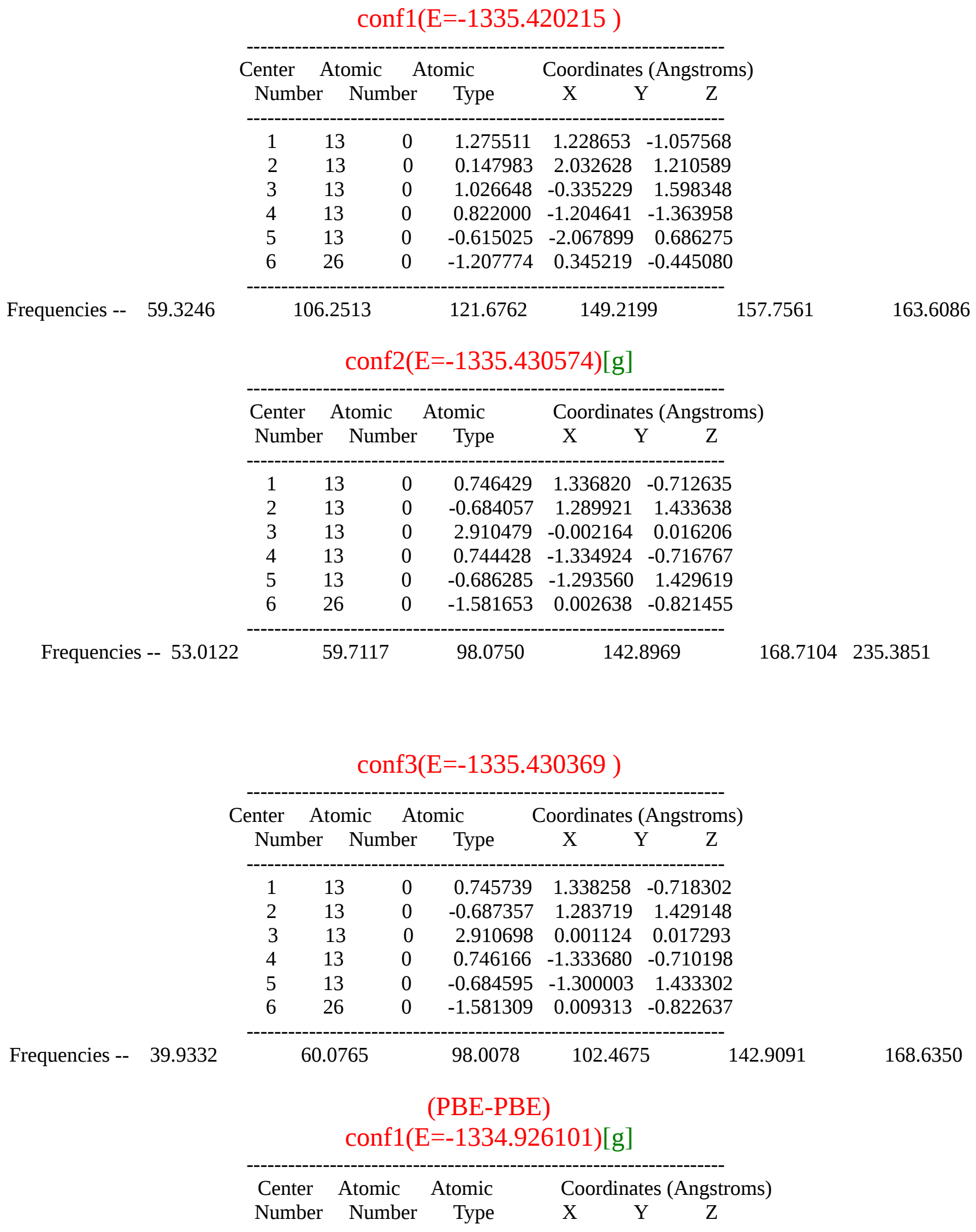




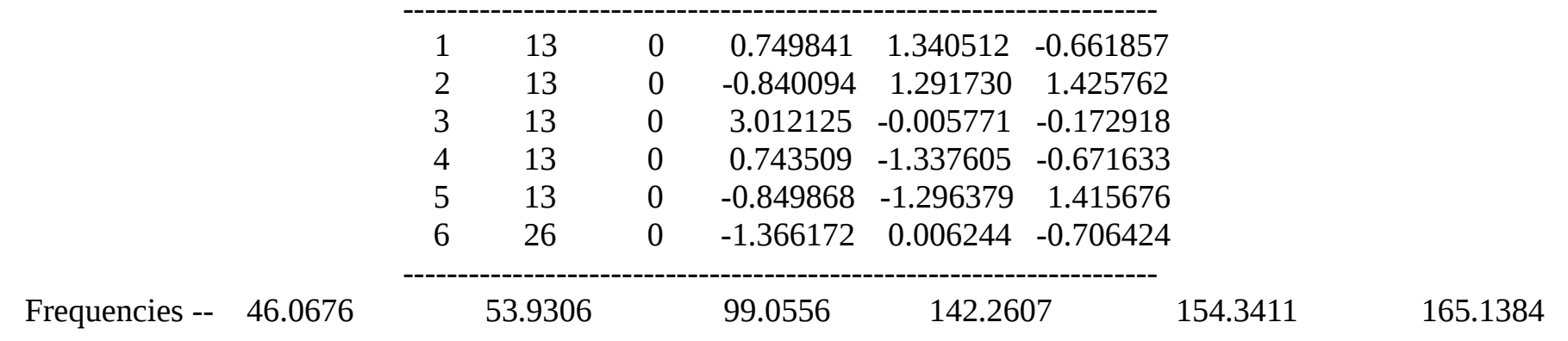

Frequencies -- 78.3169

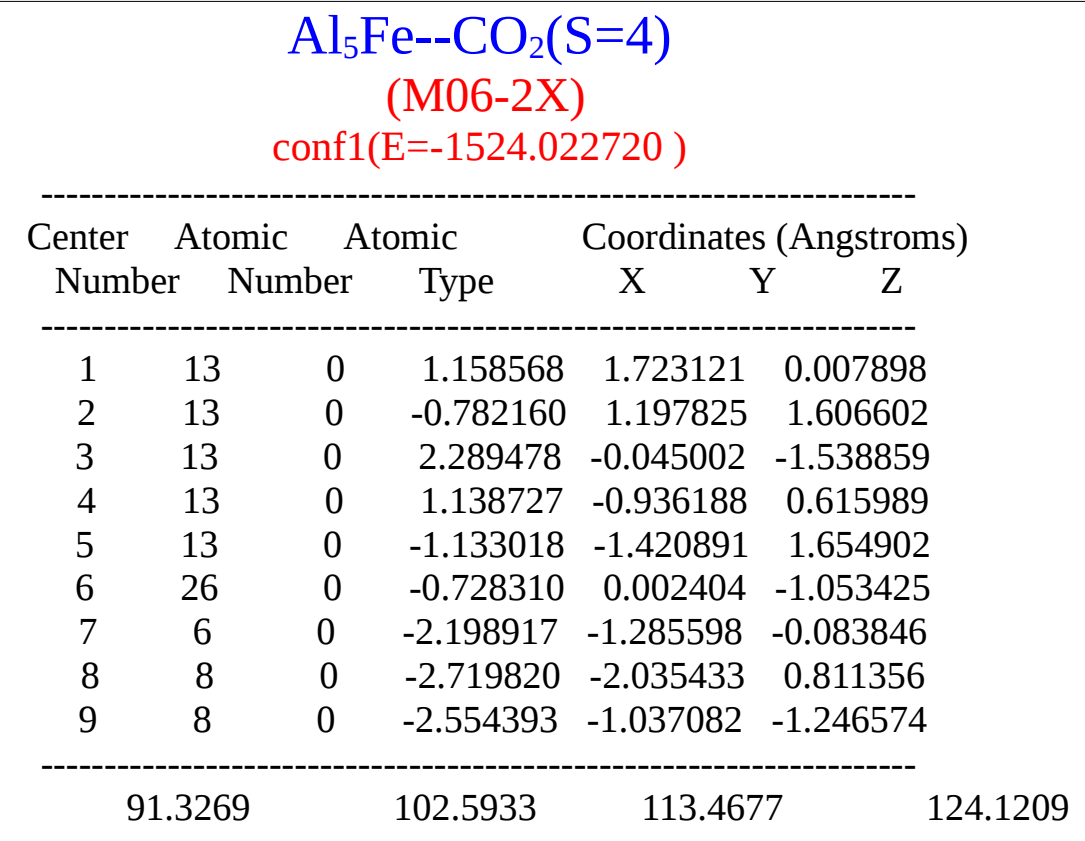

162.6338

$$
\text { Conf2(E=-1524.012846) }
$$

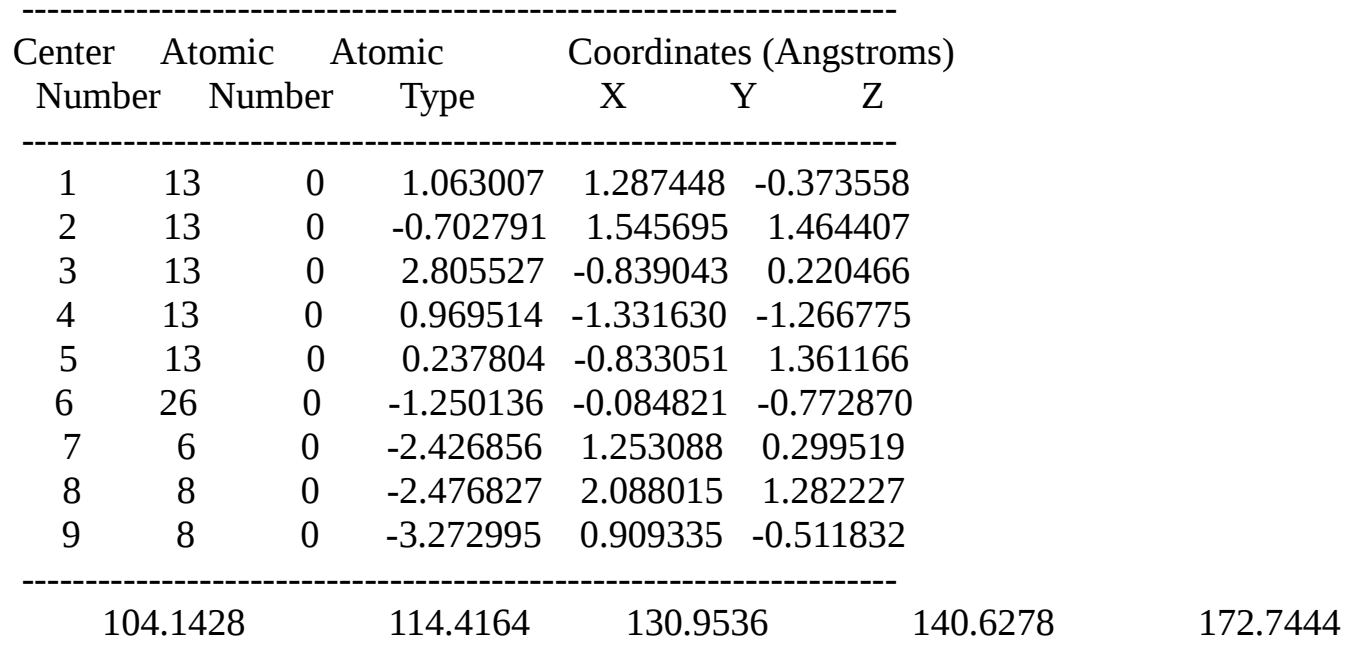

Frequencies -- 51.8779

Conf3(E=-1524.023482)
Center Atomic Atomic Coordinates (Angstroms)




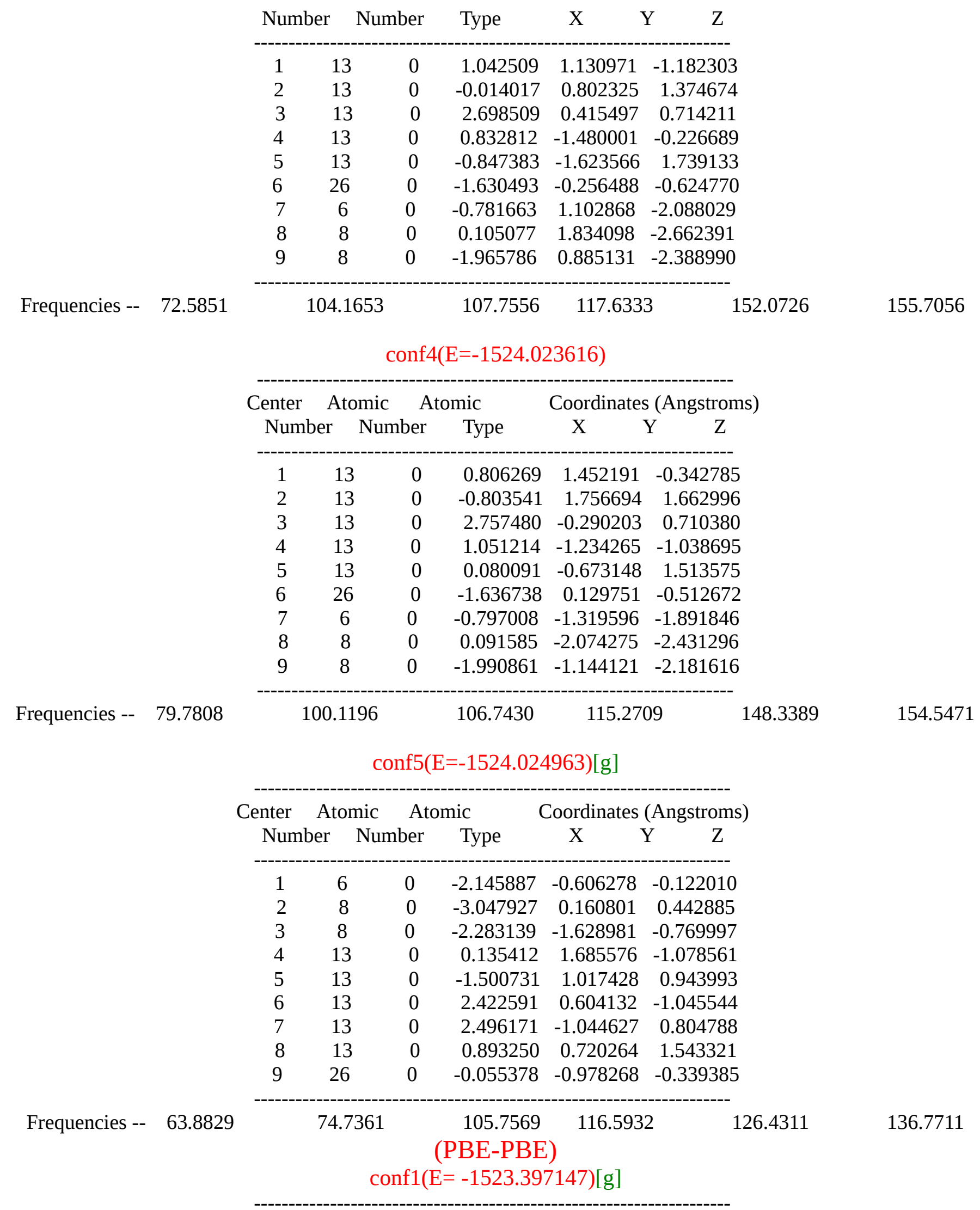




\begin{tabular}{|c|c|c|c|c|c|}
\hline \multirow{2}{*}{$\begin{array}{c}\text { Center } \\
\text { Number }\end{array}$} & \multirow{2}{*}{$\begin{array}{l}\text { Atomic } \\
\text { Number }\end{array}$} & \multirow{2}{*}{$\begin{array}{c}\text { Atomic } \\
\text { Type }\end{array}$} & \multicolumn{2}{|c|}{ Coordinates (Angstroms) } & \\
\hline & & & X $\quad Y$ & $Y \quad Z$ & \\
\hline 1 & 6 & -1.933502 & -0.910367 & -0.186822 & \\
\hline 2 & 8 & -2.927973 & -0.350941 & 0.470438 & \\
\hline 3 & 8 & -1.922728 & -1.890582 & -0.933402 & \\
\hline 4 & 13 & -0.013528 & 1.881625 & -0.842939 & \\
\hline 5 & 13 & -1.584891 & 0.831518 & 1.066197 & \\
\hline 6 & 13 & 2.231847 & 0.766381 & -1.139090 & \\
\hline 7 & 13 & 2.527892 & -1.009134 & 0.663063 & \\
\hline 8 & 13 & 0.840844 & 0.596543 & 1.604286 & \\
\hline 9 & 26 & 0.052932 & -0.676392 & -0.401989 & \\
\hline & 8.9170 & 74.7121 & 104.0328 & 111.9245 & 127.7899 \\
\hline
\end{tabular}

Frequencies -- 61.4022

$$
\begin{gathered}
\mathrm{Al}_{5} \mathrm{Co}(\mathrm{S}=3) \\
(\mathrm{M} 06-2 \mathrm{X}) \\
\operatorname{conf1}(\mathrm{E}=-1357.095494)[\mathrm{g}]
\end{gathered}
$$

Center Atomic Atomic Coordinates (Angstroms)

Number Number Type $\quad$ X $\quad$ Y Z

Frequencies -- 59.0825

$\begin{array}{lllrrr}1 & 13 & 0 & 1.308402 & -1.498602 & 0.129297 \\ 2 & 13 & 0 & 2.163807 & 0.921356 & 0.256828 \\ 3 & 13 & 0 & 0.004227 & 0.605521 & 1.521945 \\ 4 & 13 & 0 & -1.312047 & -1.495236 & 0.155488 \\ 5 & 13 & 0 & -2.172652 & 0.923781 & 0.290915 \\ 6 & 27 & 0 & 0.008903 & 0.547103 & -1.255268 \\ ------------163 & \end{array}$

192.1760

$$
\text { conf2(E=-1357.061235) }
$$

Center Atomic Atomic Coordinates (Angstroms)

Number Number Type $\quad$ X $\quad$ Y $\quad$ Z

$\begin{array}{rrrrrr}1 & 13 & 0 & 2.130069 & -1.001947 & 0.269455 \\ 2 & 13 & 0 & 1.252309 & 1.518800 & 0.064527 \\ 3 & 13 & 0 & 0.003233 & -0.690705 & 1.685902 \\ 4 & 13 & 0 & -2.134121 & -0.988757 & 0.292551 \\ 5 & 13 & 0 & -1.265281 & 1.522815 & 0.050752 \\ 6 & 27 & 0 & 0.014430 & -0.356284 & -1.263981\end{array}$

Frequencies -- 75.0382

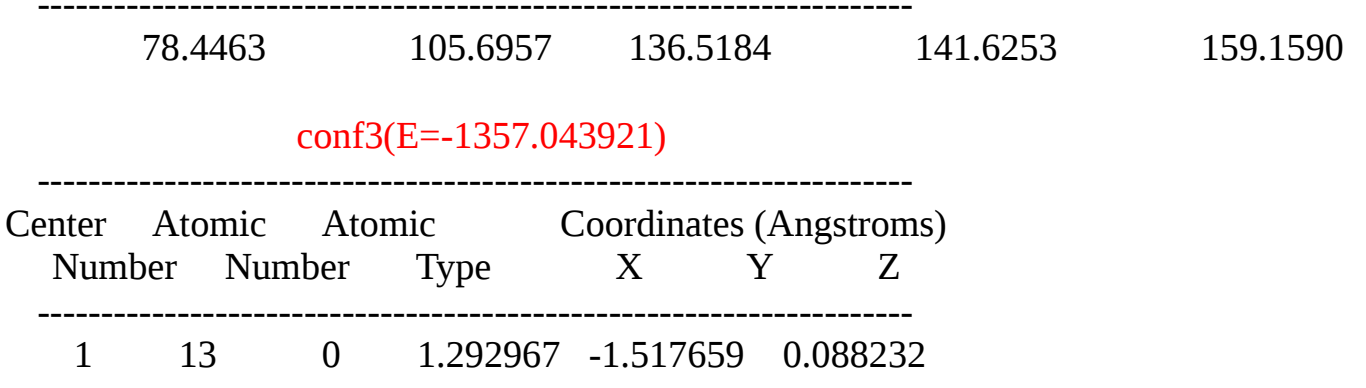




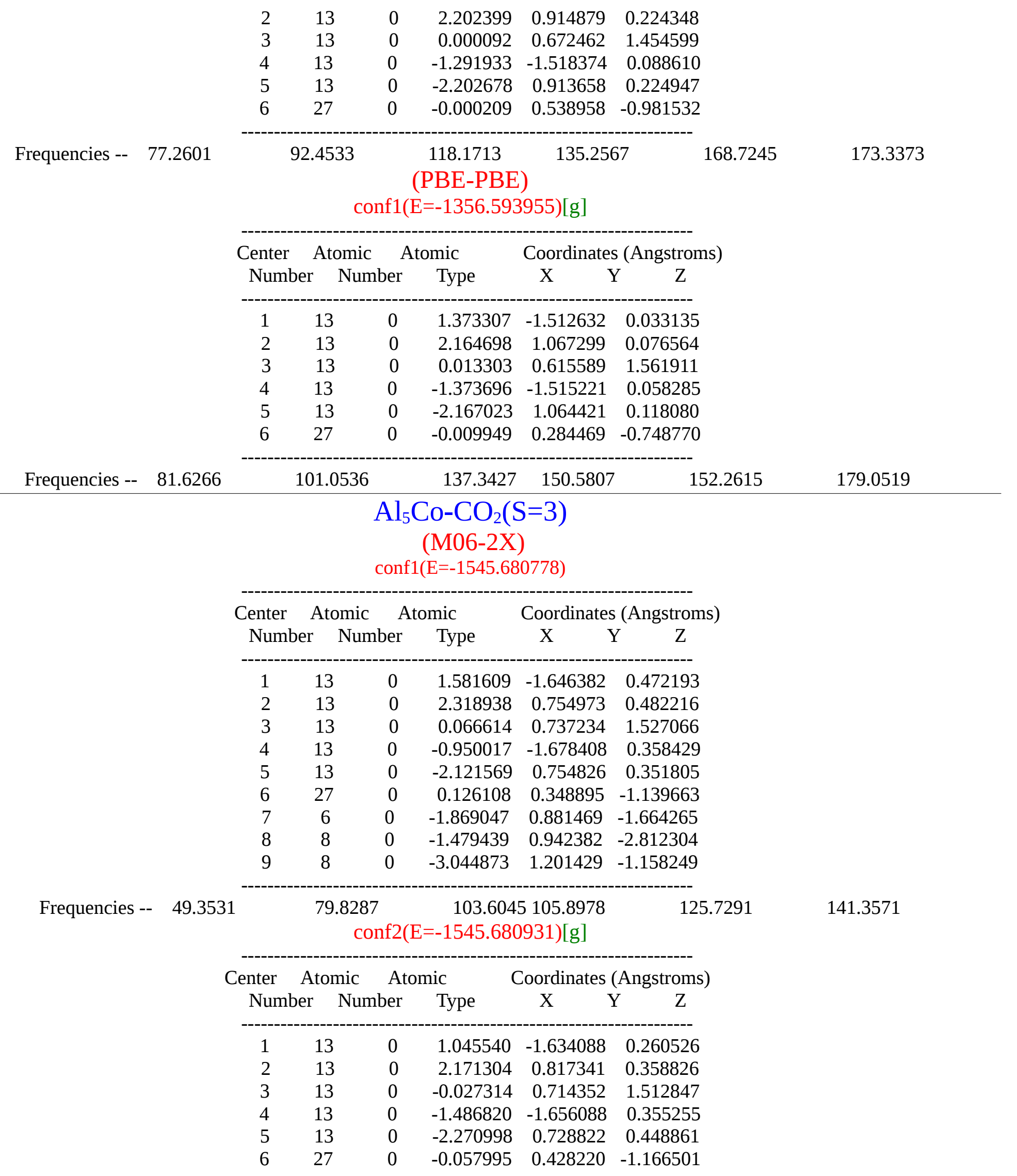




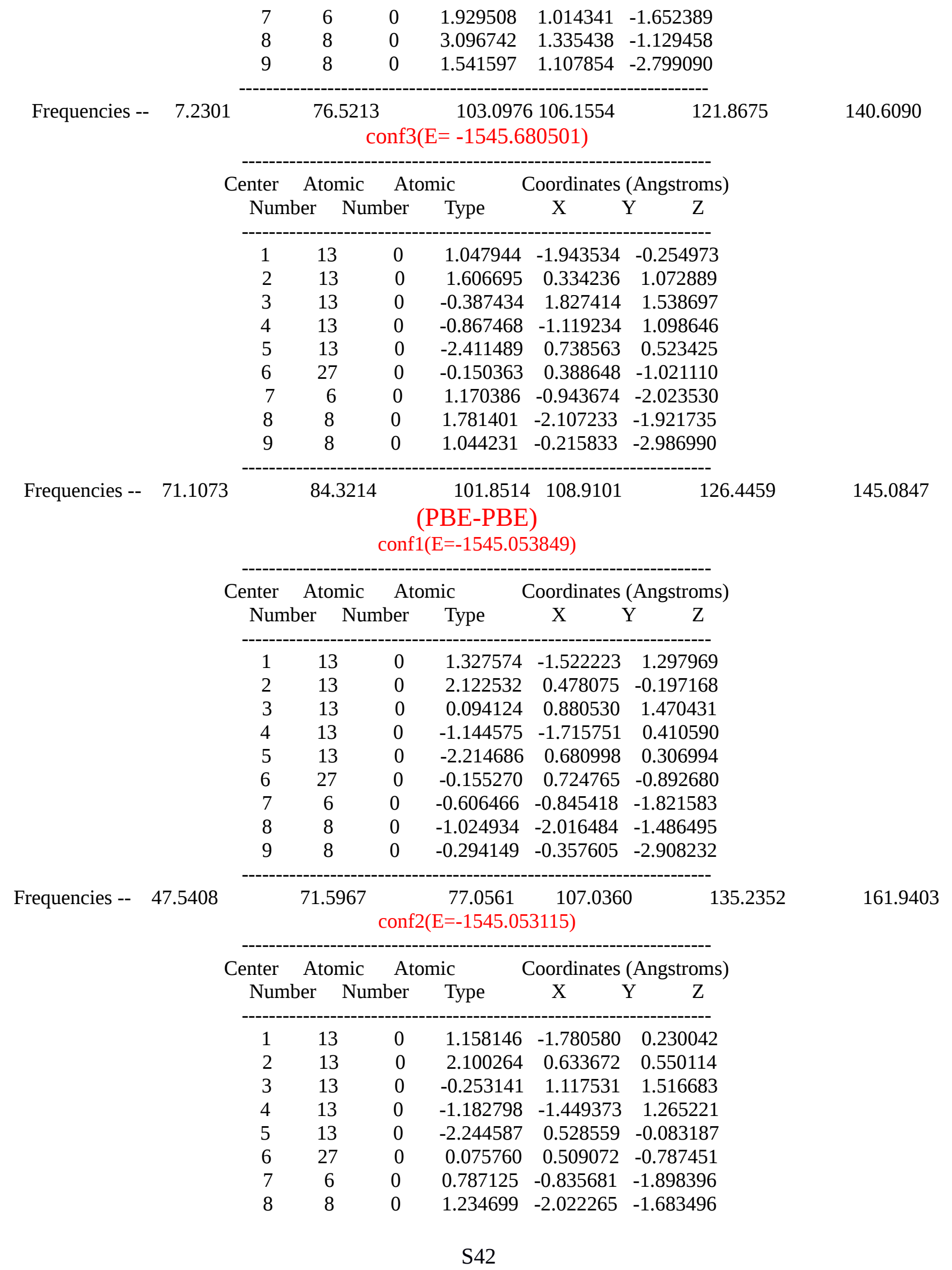




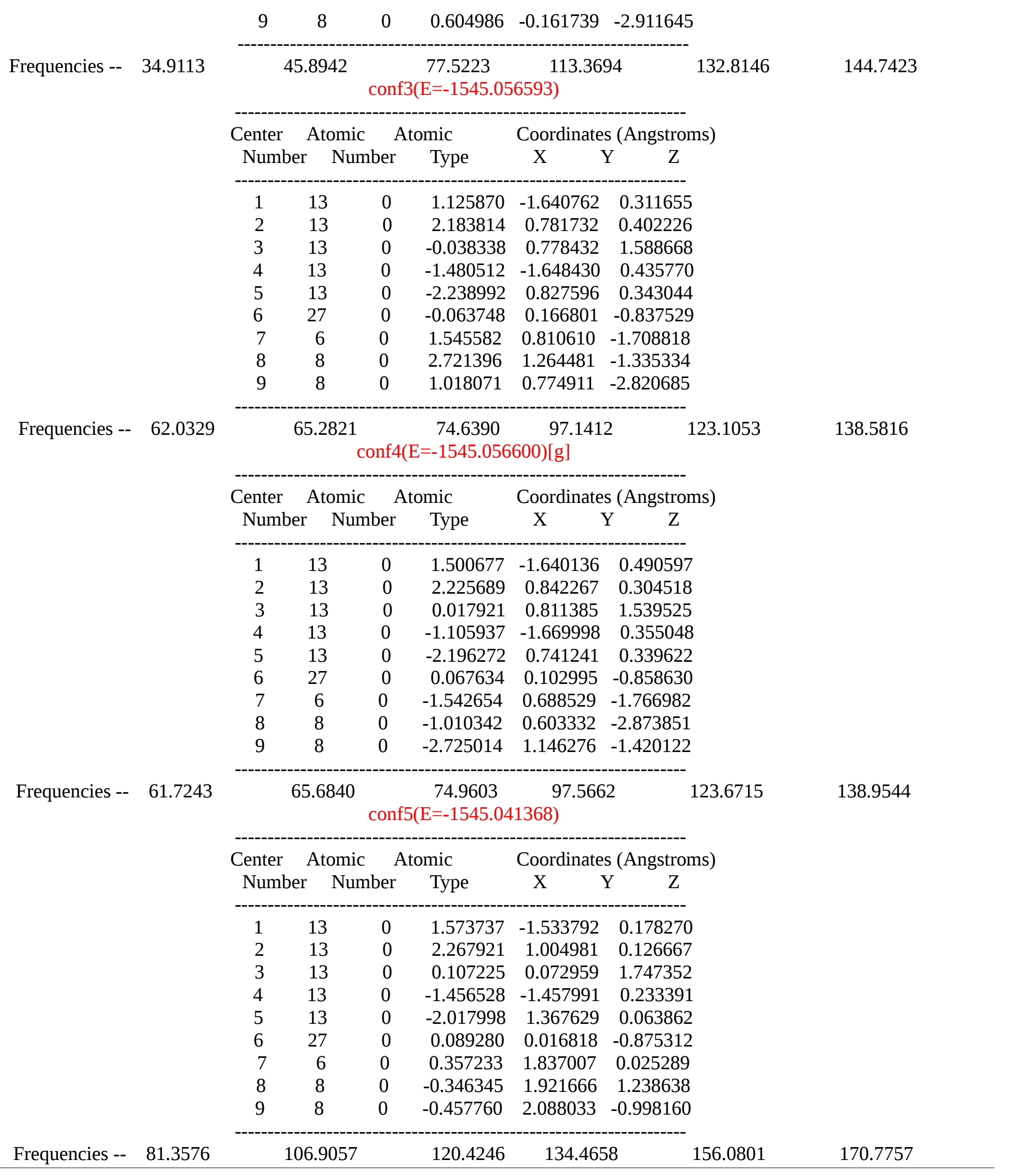




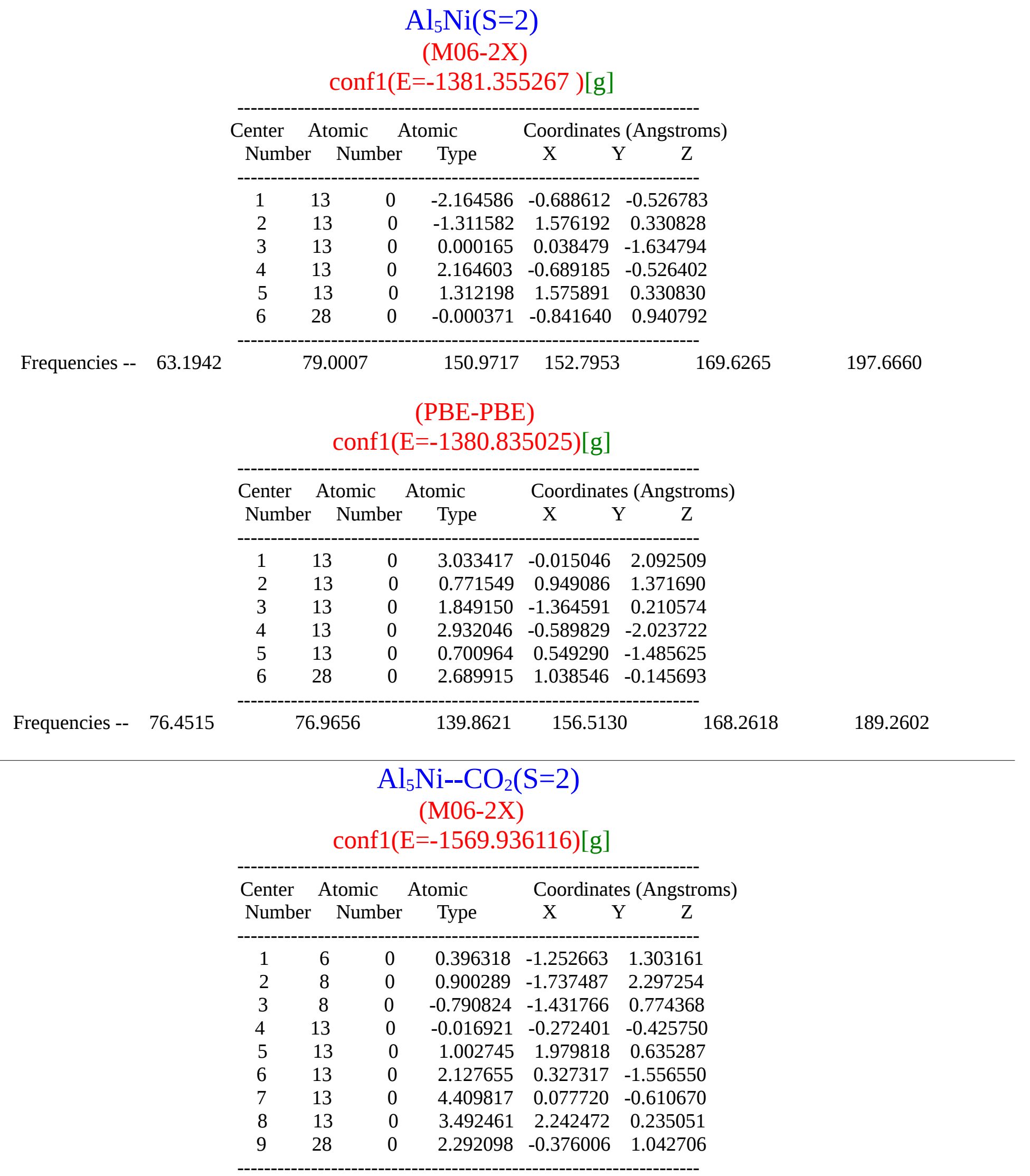




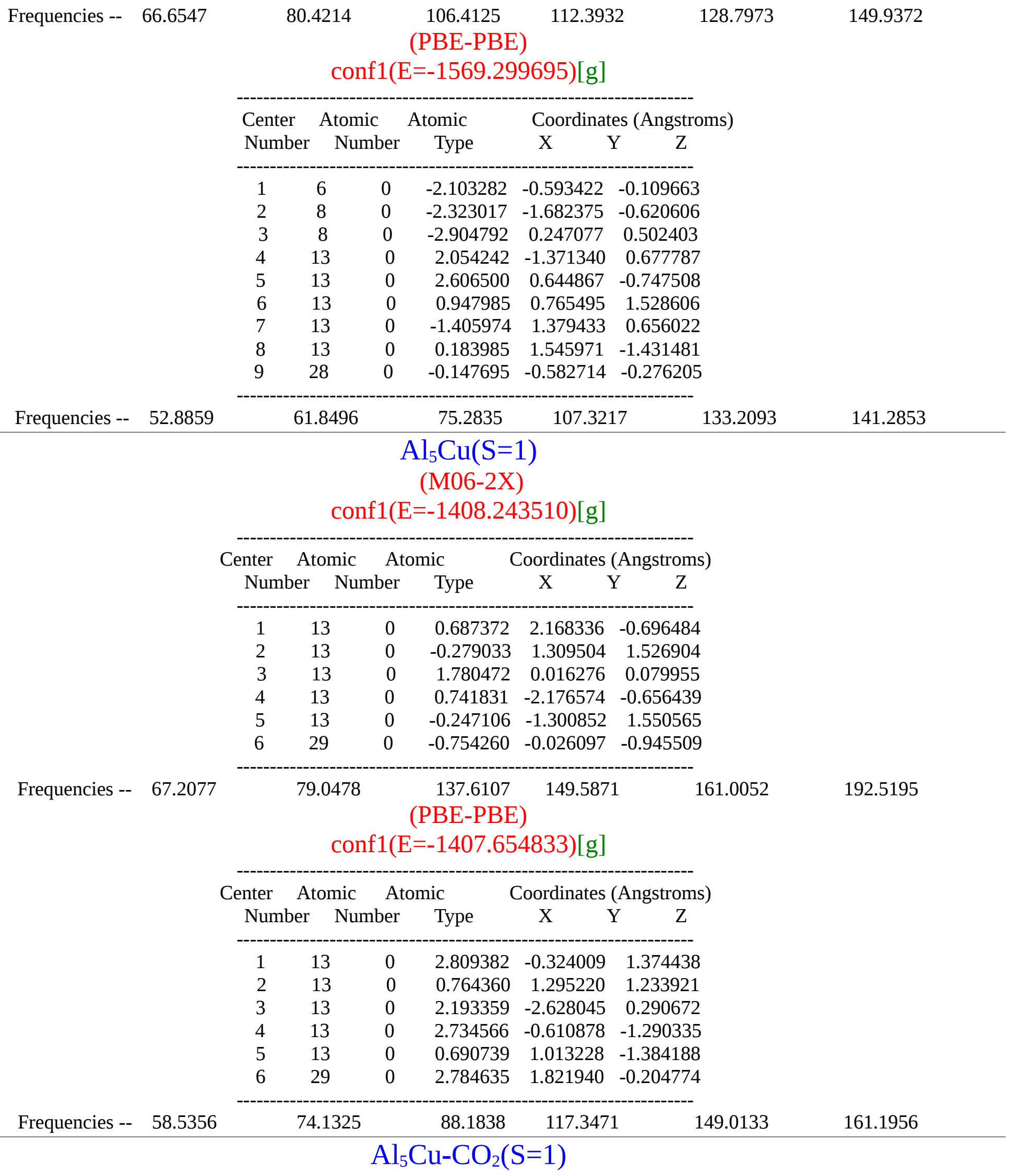




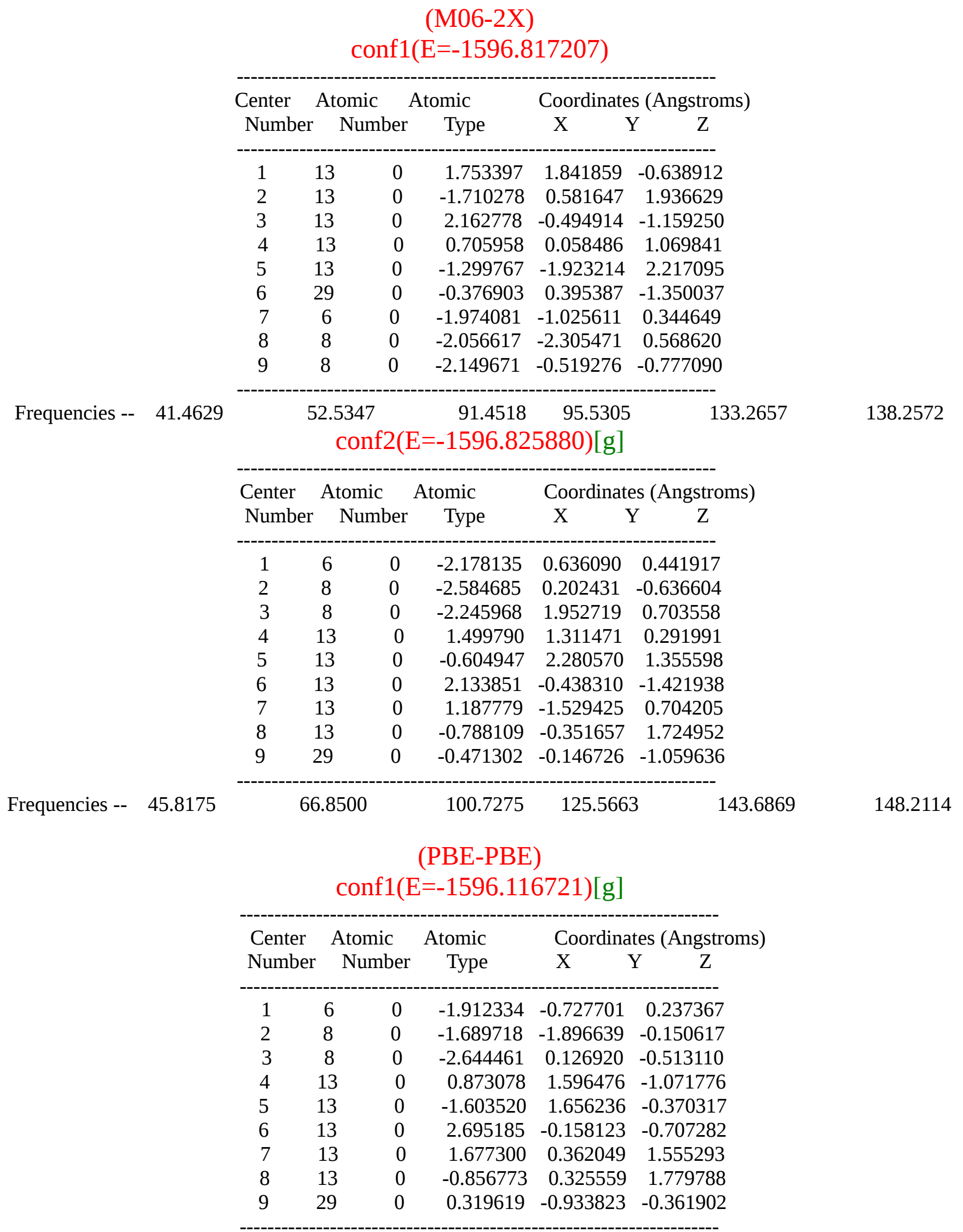




\section{$\mathrm{Al}_{5} \mathrm{Zn}(\mathrm{S}=2)$}

(M06-2X)

$\operatorname{conf1}(\mathrm{E}=-1277.460150)$

Center Atomic Atomic Coordinates (Angstroms)

Number Number Type X Y Z

Frequencies -- 32.0406

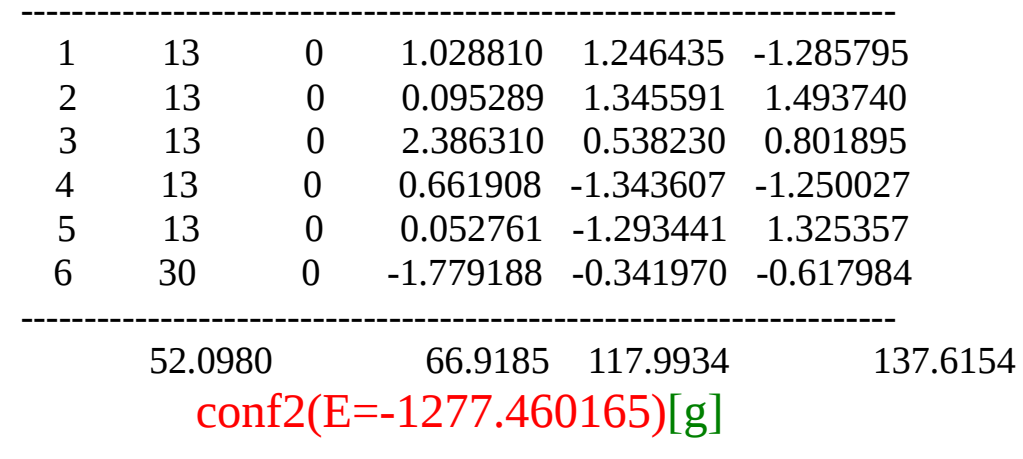

151.8548

Center Atomic Atomic Coordinates (Angstroms)

$\begin{array}{rrrrrr}\text { Number } & \text { Number } & \text { Type } & \text { X } & \text { Y } & \text { Z } \\ -1 & 13 & 0 & 0.909108 & 1.463741 & -1.108190 \\ 2 & 13 & 0 & -0.273736 & 1.407967 & 1.224112 \\ 3 & 13 & 0 & 2.563283 & 0.071617 & 0.314181 \\ 4 & 13 & 0 & 1.063487 & -1.463237 & -1.018269 \\ 5 & 13 & 0 & 0.054254 & -1.211820 & 1.411839 \\ 6 & 30 & 0 & -1.870506 & -0.117029 & -0.356486\end{array}$

Frequencies -- 32.9433

49.1417

$65.3680 \quad 118.2074$

152.9090

(PBE-PBE)

$$
\operatorname{conf1}(\mathrm{E}=-1277.392125) \text { [g] }
$$

Center Atomic Atomic Coordinates (Angstroms)

\begin{tabular}{rrrrrr} 
Number & Number & Type & X & Y & Z \\
\hline 1 & 13 & 0 & 3.096808 & -0.397179 & 1.529549 \\
2 & 13 & 0 & 0.835108 & 0.917931 & 1.270215 \\
3 & 13 & 0 & 1.606124 & -2.046616 & 0.280115 \\
4 & 13 & 0 & 3.031520 & -0.764990 & -1.401534 \\
5 & 13 & 0 & 0.776007 & 0.585461 & -1.375194 \\
6 & 30 & 0 & 2.631473 & 2.272850 & -0.283417
\end{tabular}

Frequencies -- 21.7719

53.5419

$65.5222 \quad 120.5558$

138.1514

155.9086

$$
\mathrm{Al}_{5} \mathrm{Zn}--\mathrm{CO}_{2}(\mathrm{~S}=2)
$$

(M06-2X)

Conf1(E=-1466.042859)[g] 


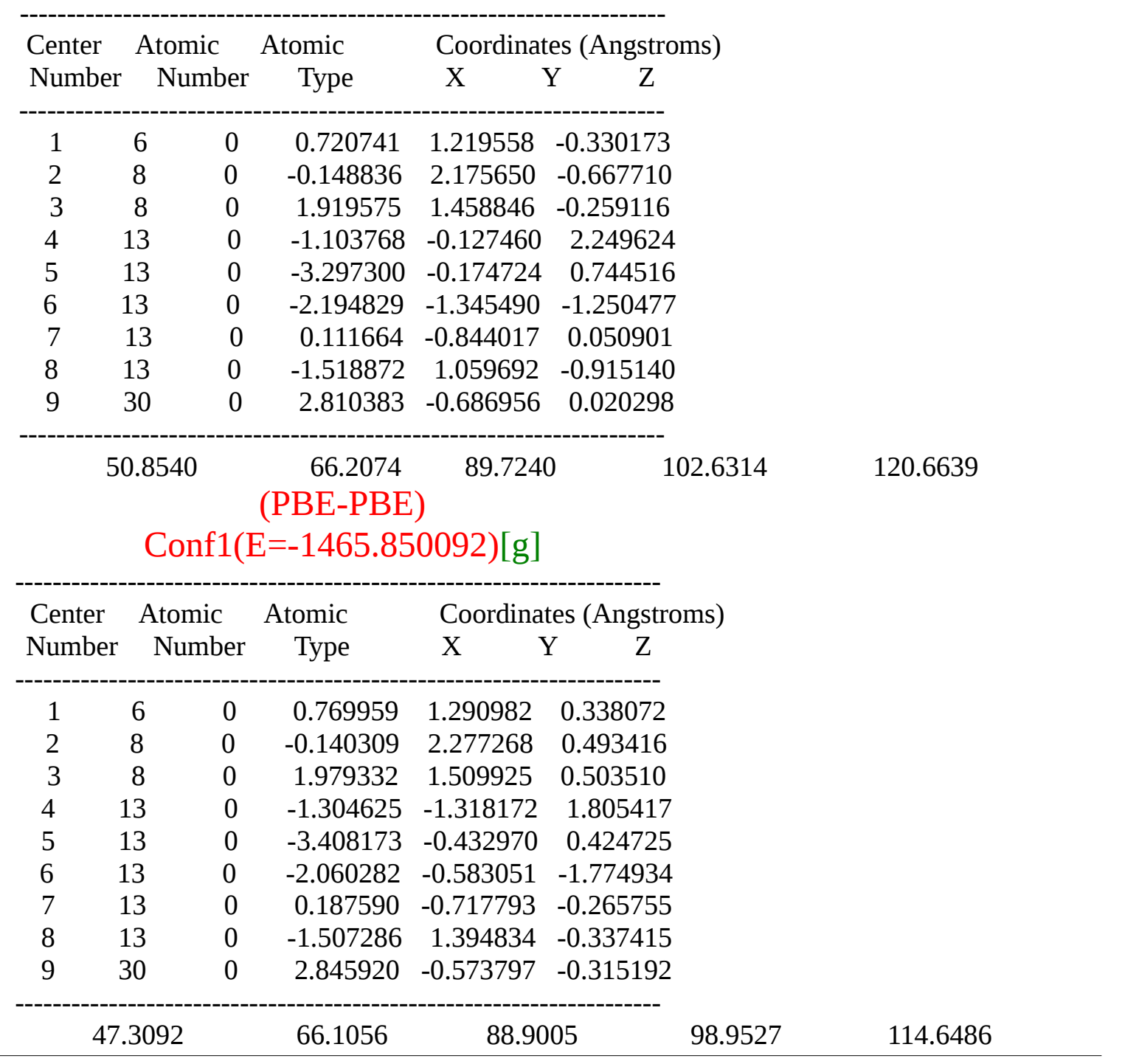




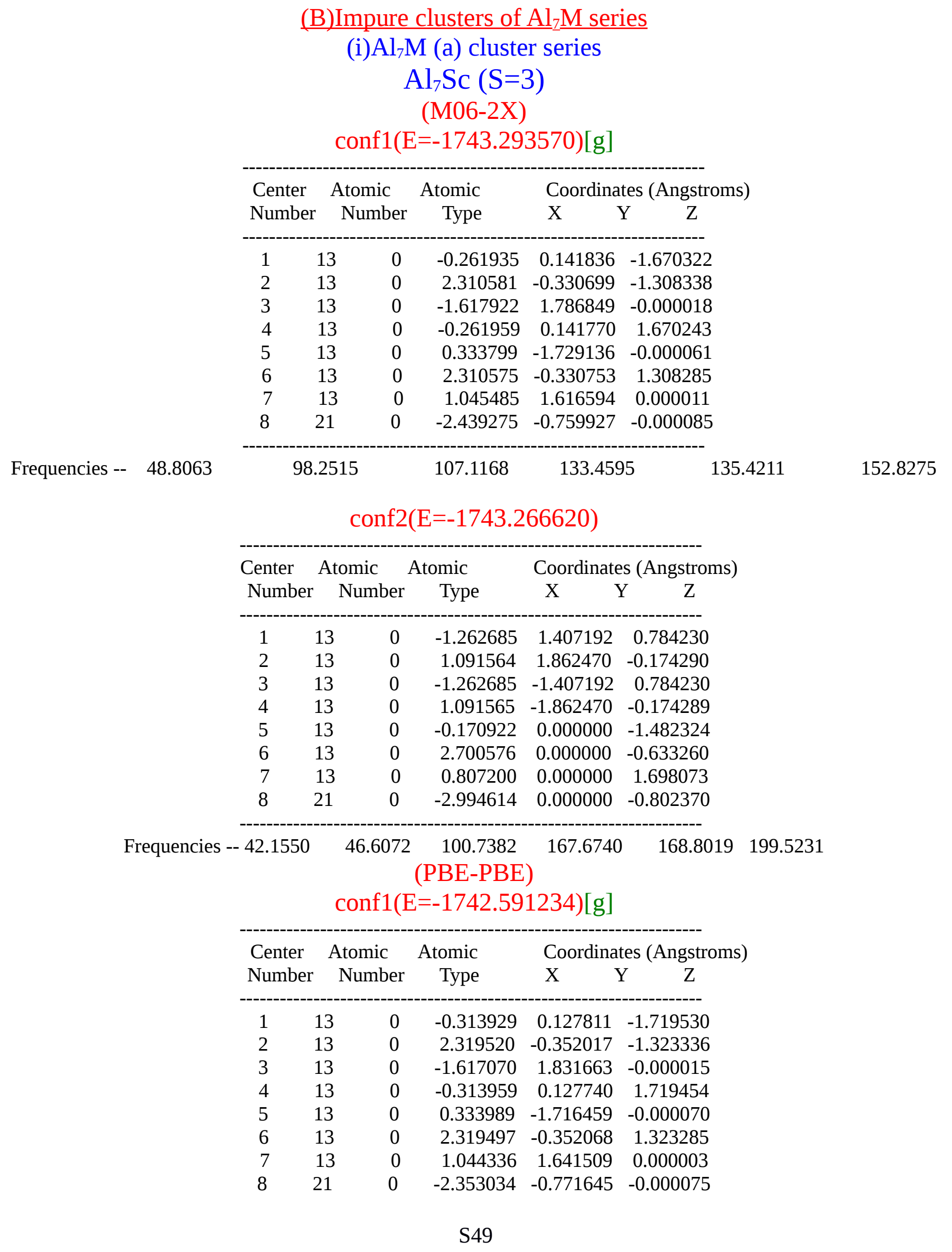




\begin{tabular}{lllll}
117.4163 & 130.3881 & 144.4887 & 145.1126 & 166.4672 \\
\hline
\end{tabular}

$$
\begin{gathered}
\mathrm{Al}_{7} \mathrm{Sc}-\mathrm{CO}_{2}(\mathrm{~S}=3) \\
(\mathrm{M} 06-2 \mathrm{X}) \\
\operatorname{conf1}(\mathrm{E}=-1931.927104)[\mathrm{g}]
\end{gathered}
$$

Center Atomic Atomic Coordinates (Angstroms)

\begin{tabular}{|c|c|c|c|}
\hline Center & Atomic & Atomic & Coordinates (Angstroms) \\
\hline Number & Number & Type & $\begin{array}{lll}X & Y & Z\end{array}$ \\
\hline
\end{tabular}

\begin{tabular}{rrrrrr} 
Number & Number & \multicolumn{1}{c}{ Type } & \multicolumn{1}{c}{ X } & $Y$ & Y \\
\hline 1 & 13 & 0 & -0.241582 & 0.005254 & -1.625958 \\
2 & 13 & 0 & 2.387009 & -0.227811 & -1.401545 \\
3 & 13 & 0 & -1.444460 & 1.674669 & 0.133377 \\
4 & 13 & 0 & -0.203725 & -0.019545 & 1.719942 \\
5 & 13 & 0 & 0.531434 & -1.780139 & 0.006619 \\
6 & 13 & 0 & 2.378601 & -0.240326 & 1.254756 \\
7 & 13 & 0 & 1.106482 & 1.654654 & -0.003008 \\
8 & 21 & 0 & -2.368380 & -1.022584 & 0.072566 \\
9 & 6 & 0 & -3.306532 & 0.930195 & 0.036082 \\
10 & 8 & 0 & -3.257601 & 2.212744 & 0.058952 \\
11 & 8 & 0 & -4.243720 & 0.118183 & 0.012301
\end{tabular}

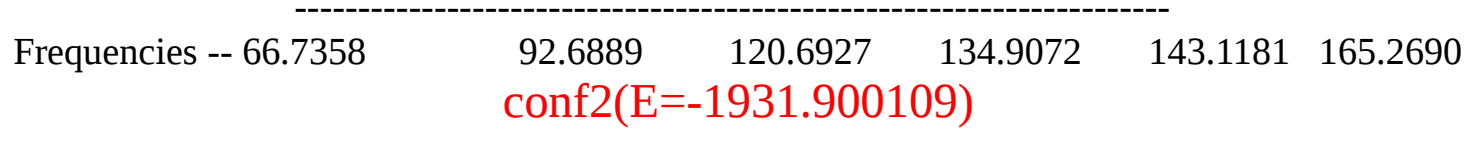

\begin{tabular}{|c|c|c|c|c|c|}
\hline 1 & 13 & 0 & -0.219714 & 0.321688 & -1.583933 \\
\hline 2 & 13 & 0 & 2.274307 & -0.329191 & -1.318382 \\
\hline 3 & 13 & 0 & -1.512392 & 2.061789 & 0.006131 \\
\hline 4 & 13 & 0 & -0.224065 & 0.302407 & 1.578370 \\
\hline 5 & 13 & 0 & 0.365762 & -1.731183 & -0.015624 \\
\hline 6 & 13 & 0 & 2.269261 & -0.345600 & 1.307824 \\
\hline 7 & 13 & 0 & 1.137449 & 1.851353 & 0.008090 \\
\hline 8 & 21 & 0 & -2.421437 & -0.538286 & -0.009764 \\
\hline 9 & 6 & 0 & -1.534486 & -2.520215 & -0.013662 \\
\hline 10 & 8 & 0 & -2.774842 & -2.643391 & -0.008320 \\
\hline 11 & 8 & 0 & -0.596245 & -3.374151 & -0.017507 \\
\hline
\end{tabular}

Frequencies -- 14.5939

\begin{tabular}{|c|c|c|c|c|}
\hline \multirow{2}{*}{$\begin{array}{l}\text { Center } \\
\text { Numbe }\end{array}$} & Atomic & Atomic & \multicolumn{2}{|c|}{ Coordinates (Angstroms } \\
\hline & r Numbe & er Type & $\mathrm{X}$ & $\mathrm{Y} \quad \mathrm{Z}$ \\
\hline 1 & 13 & -0.259568 & 0.050832 & -1.694078 \\
\hline 2 & 13 & 2.223671 & -0.462421 & -0.999286 \\
\hline 3 & 13 & -1.383600 & 1.830852 & 0.293685 \\
\hline 4 & 13 & 0.016669 & 0.054299 & 1.812127 \\
\hline
\end{tabular}

$\begin{array}{cccc}84.7258 & 105.9064 & 108.8601 & 134.8136\end{array}$




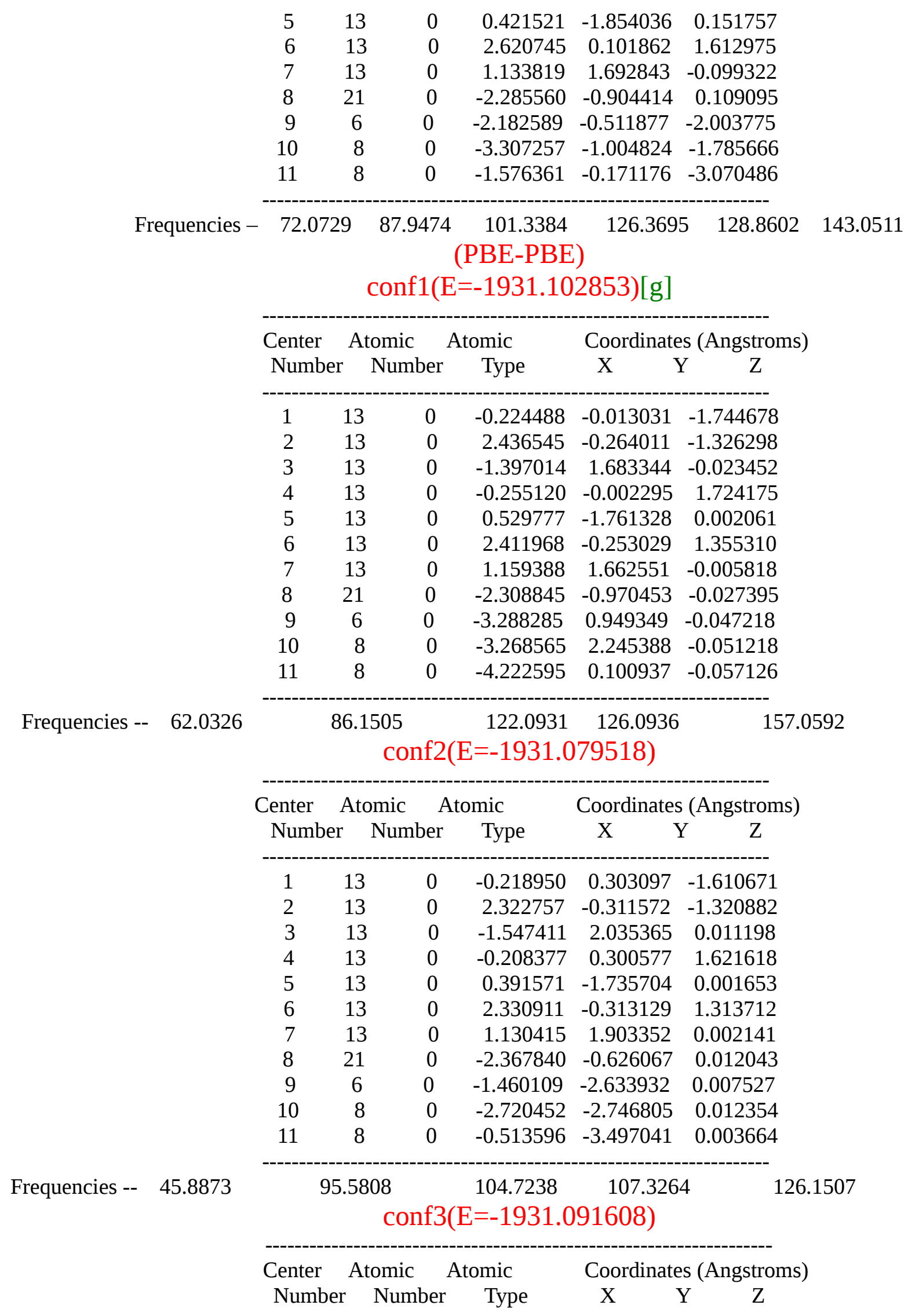

158.9217 


\begin{tabular}{|c|c|c|c|c|c|}
\hline 1 & 13 & 0 & -0.090034 & 0.171292 & -2.091966 \\
\hline 2 & 13 & 0 & 2.506346 & -0.035434 & -1.573469 \\
\hline 3 & 13 & 0 & -1.283063 & 1.661441 & -0.281480 \\
\hline 4 & 13 & 0 & -0.288435 & 0.007412 & 1.725496 \\
\hline 5 & 13 & 0 & 0.464972 & -1.571802 & -0.269577 \\
\hline 6 & 13 & 0 & 2.358263 & -0.434722 & 1.230884 \\
\hline 7 & 13 & 0 & 1.226108 & 1.654338 & 0.023201 \\
\hline 8 & 21 & 0 & -2.269115 & -0.909997 & -0.136079 \\
\hline 9 & 6 & 0 & -2.226482 & -0.707528 & 1.992822 \\
\hline 10 & 8 & 0 & -3.343917 & -1.237974 & 1.716455 \\
\hline 11 & 8 & 0 & -1.659379 & -0.390184 & 3.101633 \\
\hline
\end{tabular}

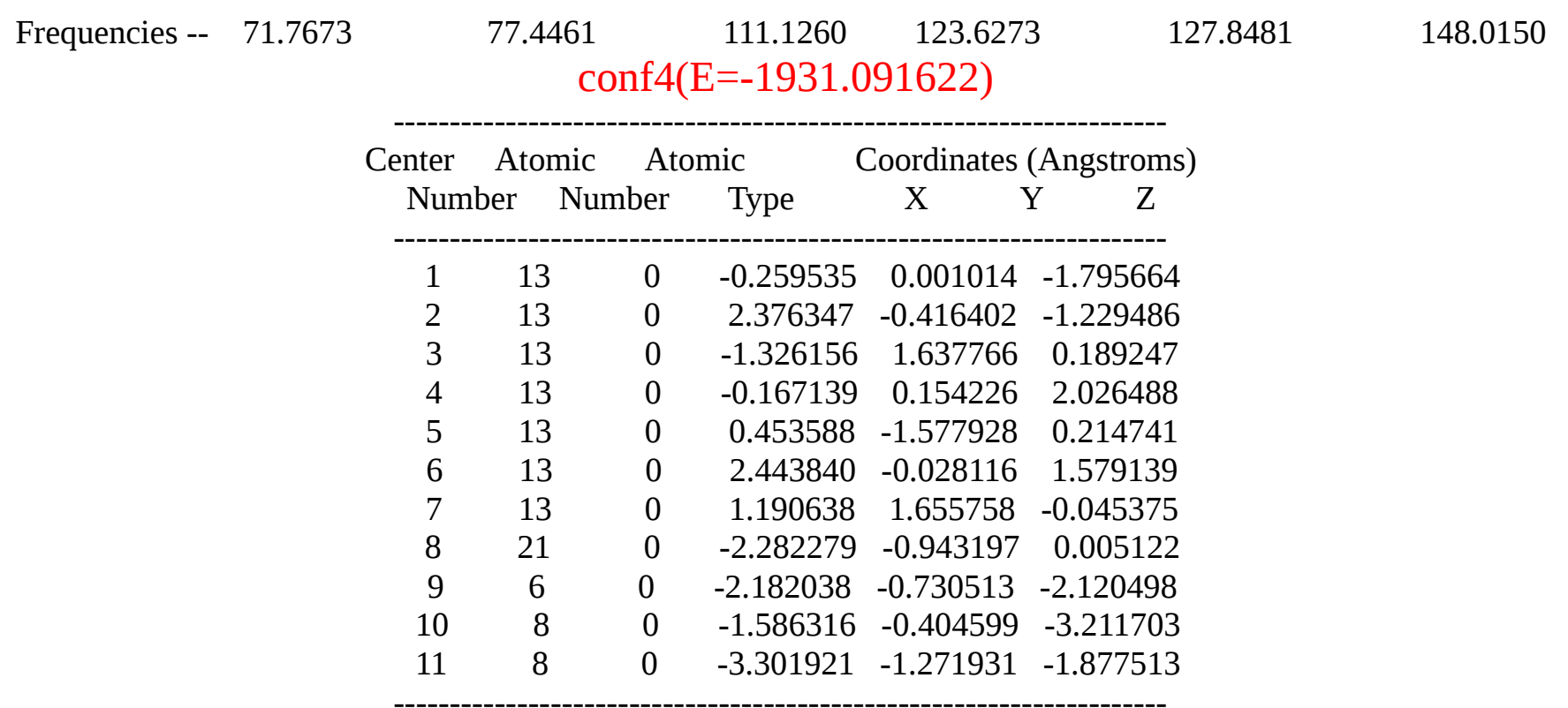

Frequencies -- 71.3596

78.2445

$111.2721 \quad 123.8269$ 127.6574

148.0538

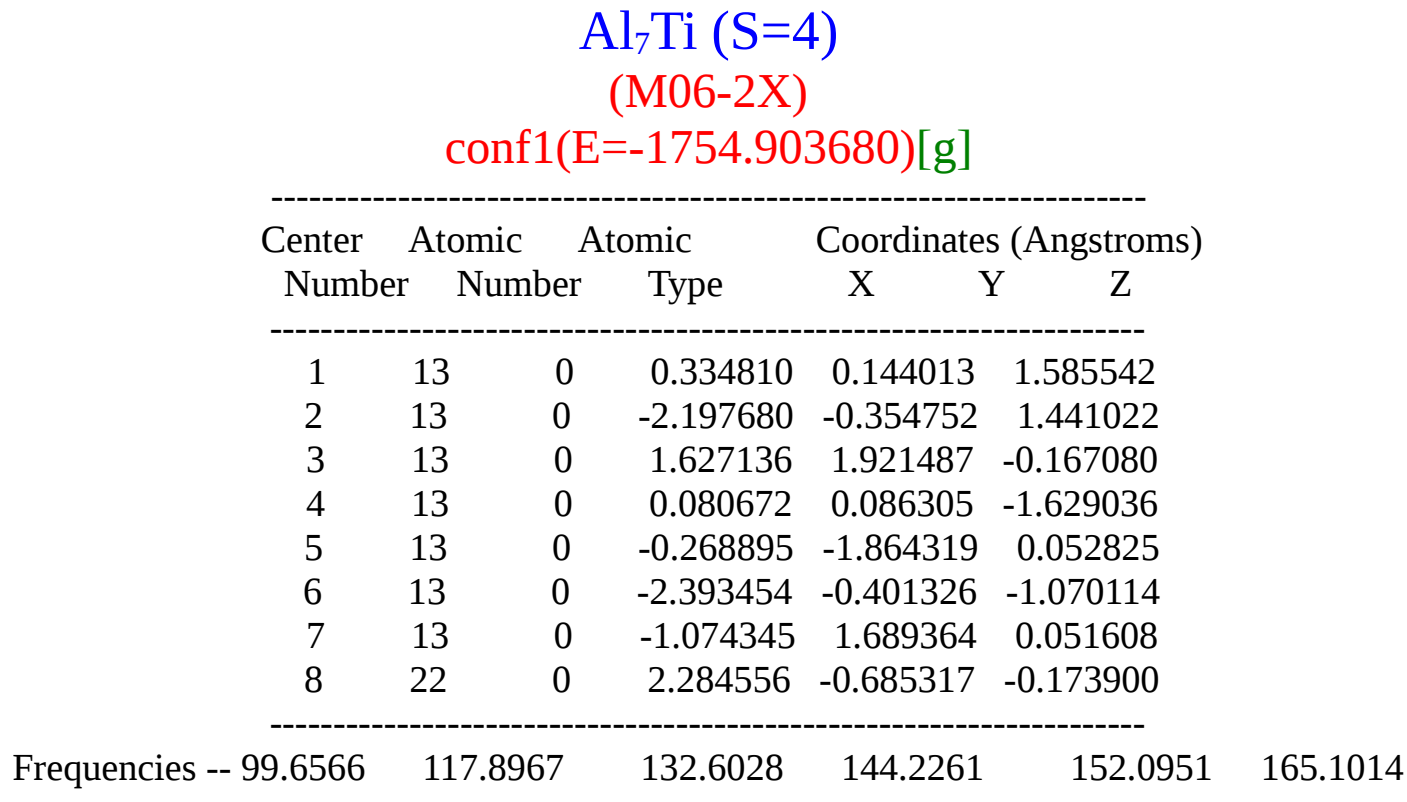




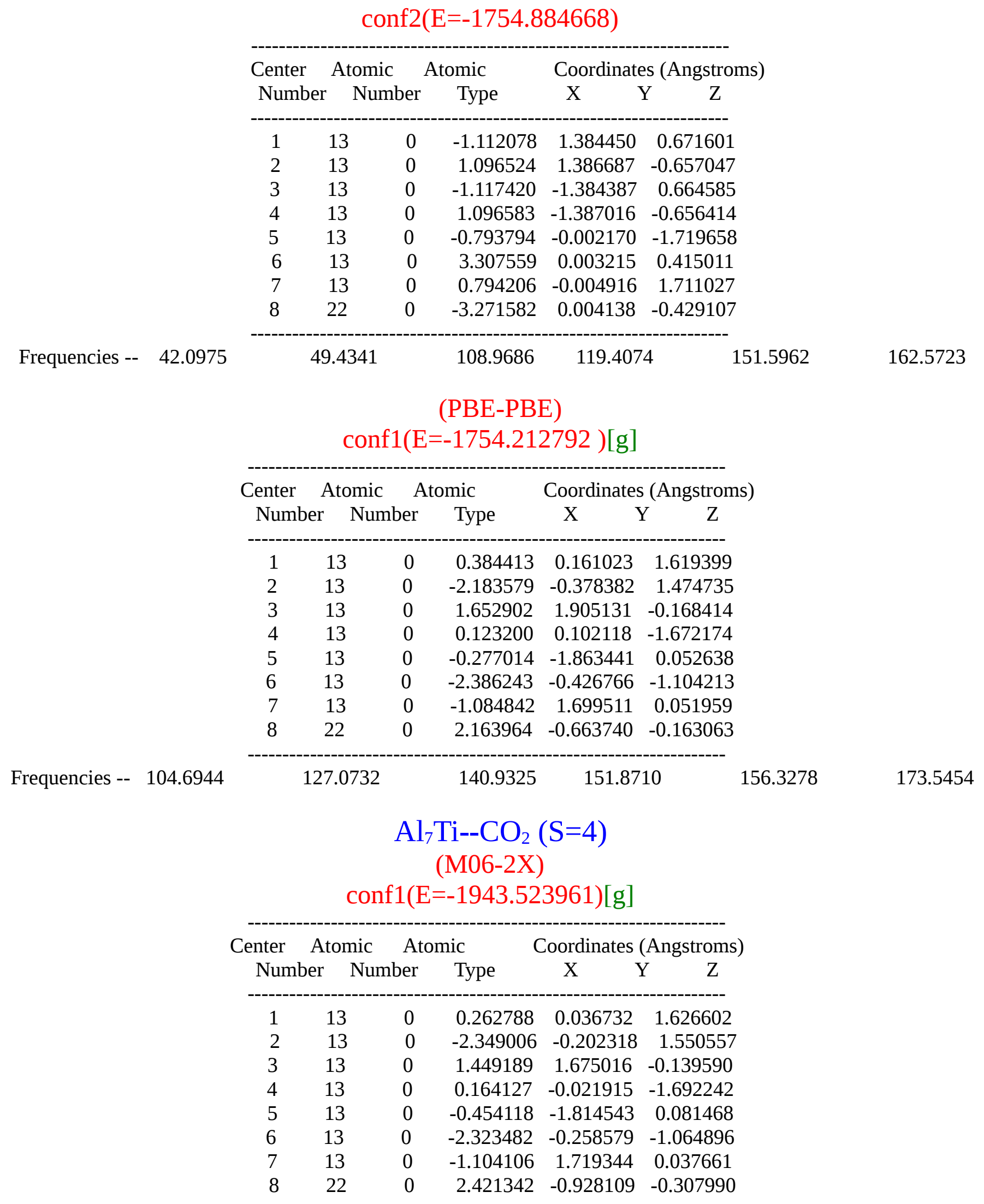




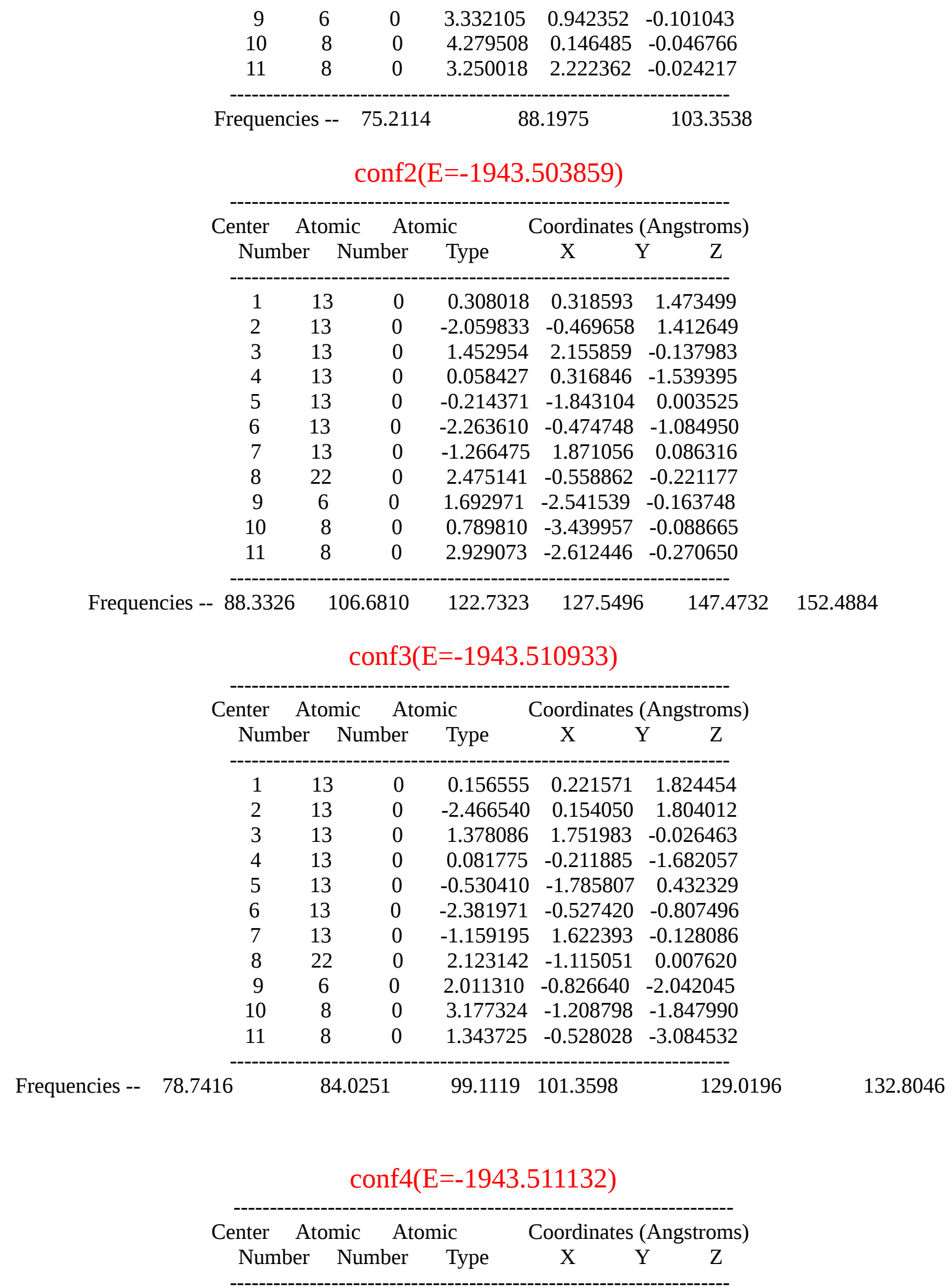




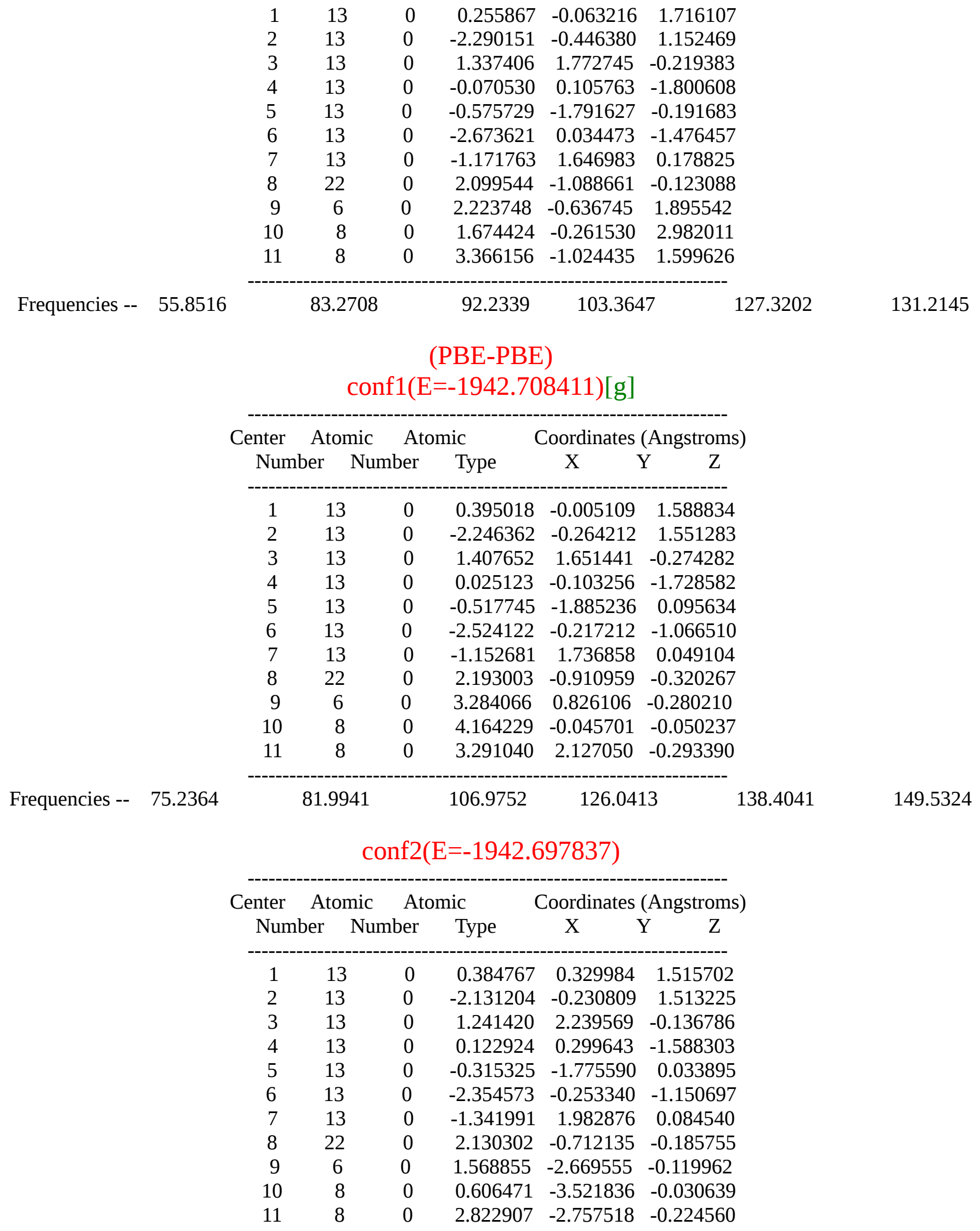




\begin{tabular}{|c|c|c|c|c|c|c|c|}
\hline \multirow{2}{*}{ Frequencies -- } & \multirow[b]{2}{*}{72.1443} & & & & & & \\
\hline & & & 3.0390 & 93.4015 & 113.9310 & 134.6730 & 141.3993 \\
\hline \multicolumn{8}{|c|}{ conf3(E=-1942.705697) } \\
\hline & & Center & \multirow{2}{*}{$\begin{array}{c}\text { Atomic } \\
\text { Number }\end{array}$} & Atomic & \multicolumn{2}{|c|}{ Coordinates (Angstroms) } & \\
\hline & & Number & & Type & $\begin{array}{ll}\mathrm{X} & \mathrm{Y}\end{array}$ & $\mathrm{Z}$ & \\
\hline & & 1 & 13 & 0.341961 & 0.154220 & 1.773752 & \\
\hline & & 2 & 13 & -2.238903 & -0.071369 & 1.742080 & \\
\hline & & 3 & 13 & 1.485958 & 1.752065 & -0.084235 & \\
\hline & & 4 & 13 & 0.020181 & -0.038169 & -1.825848 & \\
\hline & & 5 & 13 & -0.462872 & -1.816524 & 0.191471 & \\
\hline & & 6 & 13 & -2.465796 & -0.503551 & -0.957827 & \\
\hline & & 7 & 13 & -1.120666 & 1.655272 & -0.041331 & \\
\hline & & 8 & 22 & 2.008423 & $-0.881301-$ & -0.152781 & \\
\hline & & 9 & 6 & 1.938123 & $-0.760968-$ & -2.195129 & \\
\hline & & 10 & 8 & 1.301280 & -0.468846 & -3.269393 & \\
\hline & & 11 & 8 & 3.064540 & $-1.275927-$ & -1.945793 & \\
\hline \multirow{15}{*}{ Frequencies -- } & \multirow[t]{15}{*}{77.7107} & \multicolumn{2}{|c|}{100.6654} & 120.4391 & 122.3088 & 132.4376 & 153.7144 \\
\hline & & \multicolumn{5}{|c|}{$\operatorname{conf} 4(\mathrm{E}=-1942.705717)$} & \\
\hline & & \multirow{2}{*}{$\begin{array}{l}\text { Center A } \\
\text { Number }\end{array}$} & Atomic $\quad A$ & Atomic & \multicolumn{2}{|c|}{ Coordinates (Angstroms) } & \\
\hline & & & Number & Type & $\begin{array}{ll}\mathrm{X} & \mathrm{Y}\end{array}$ & & \\
\hline & & 1 & 13 & 0.310640 & 0.023584 & 1.735769 & \\
\hline & & 2 & 13 & -2.285413 & -0.473419 & 1.326252 & \\
\hline & & 3 & 13 & 1.433522 & 1.774147 & -0.279749 & \\
\hline & & 4 & 13 & -0.004045 & $0.120195-$ & -1.869708 & \\
\hline & & 5 & 13 & -0.500526 & $-1.810467-$ & -0.117336 & \\
\hline & & 6 & 13 & -2.536404 & $-0.111564-$ & -1.379353 & \\
\hline & & 7 & 13 & -1.139217 & 1.666973 & 0.136908 & \\
\hline & & 8 & 22 & 1.982309 & $-0.851935-$ & -0.234789 & \\
\hline & & 9 & 6 & 2.274177 & -0.672076 & 1.784049 & \\
\hline & & 10 & 8 & 3.346454 & -1.184445 & 1.355592 & \\
\hline & & 11 & 8 & 1.831337 & -0.351921 & 2.945083 & \\
\hline \multirow[t]{6}{*}{ Frequencies -- } & \multirow[t]{6}{*}{74.2489} & \multicolumn{2}{|c|}{100.4600} & 119.0377 & 122.0757 & 131.2358 & 153.0945 \\
\hline & & \multicolumn{5}{|c|}{$\begin{array}{c}\mathrm{Al}_{7} \mathrm{~V}(\mathrm{~S}=5) \\
(\mathrm{M} 06-2 \mathrm{X}) \\
\operatorname{conf1}(\mathrm{E}=-1768.173672)[\mathrm{g}]\end{array}$} & \\
\hline & & \multirow{2}{*}{\multicolumn{2}{|c|}{$\begin{array}{ll}\text { Center } & \text { Atomic } \\
\text { Number } & \text { Number }\end{array}$}} & Atomic & \multicolumn{2}{|c|}{ Coordinates (Angstroms) } & \\
\hline & & & & Type & X $\quad \mathrm{Y}$ & $\mathrm{Z}$ & \\
\hline & & 1 & 13 & -0.289280 & 0.139313 & 1.584435 & \\
\hline & & 2 & 13 & 1.158139 & 1.673030 & 0.049077 & \\
\hline
\end{tabular}




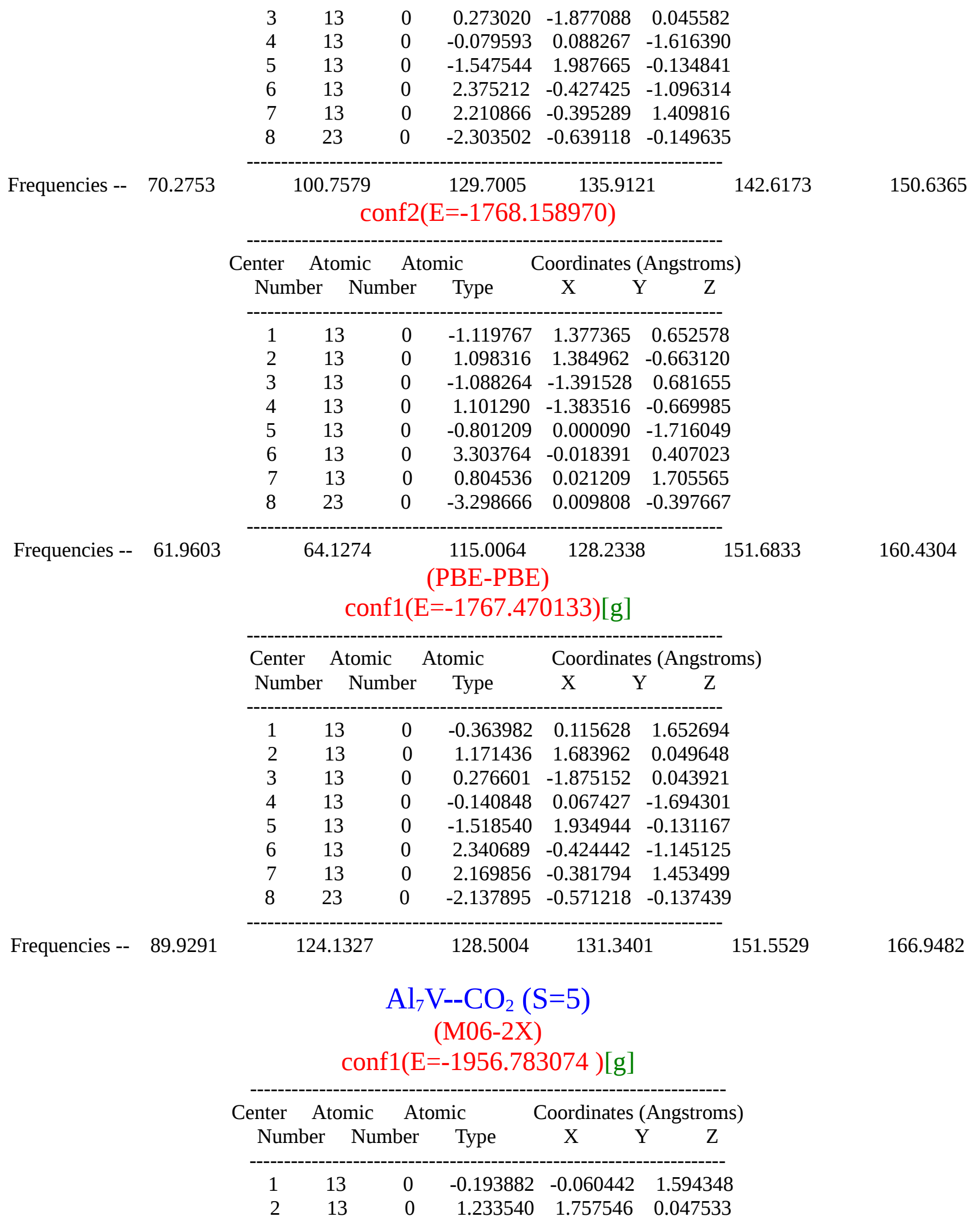




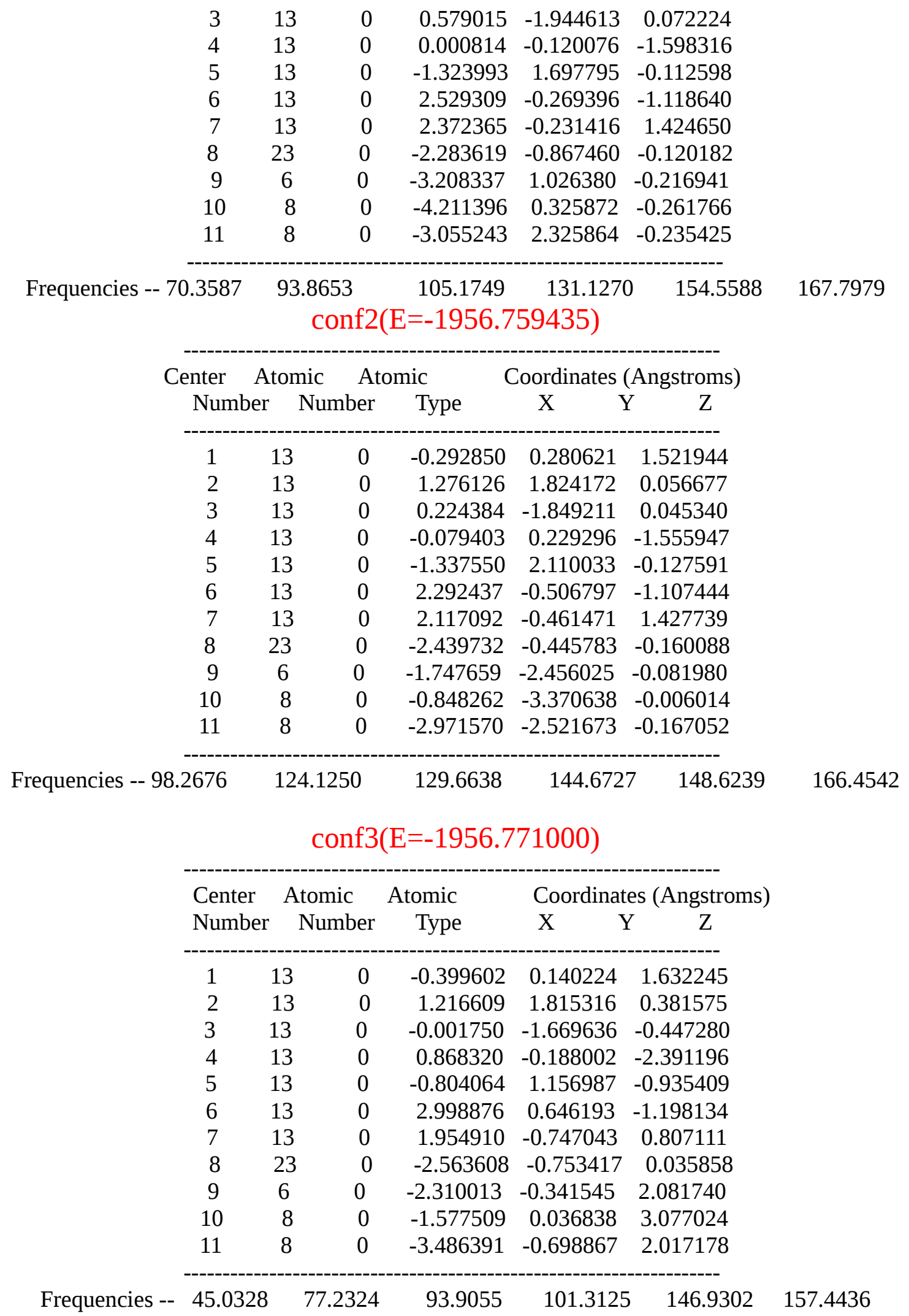

(PBE-PBE) 


\section{conf1(E=-1955.963332)}

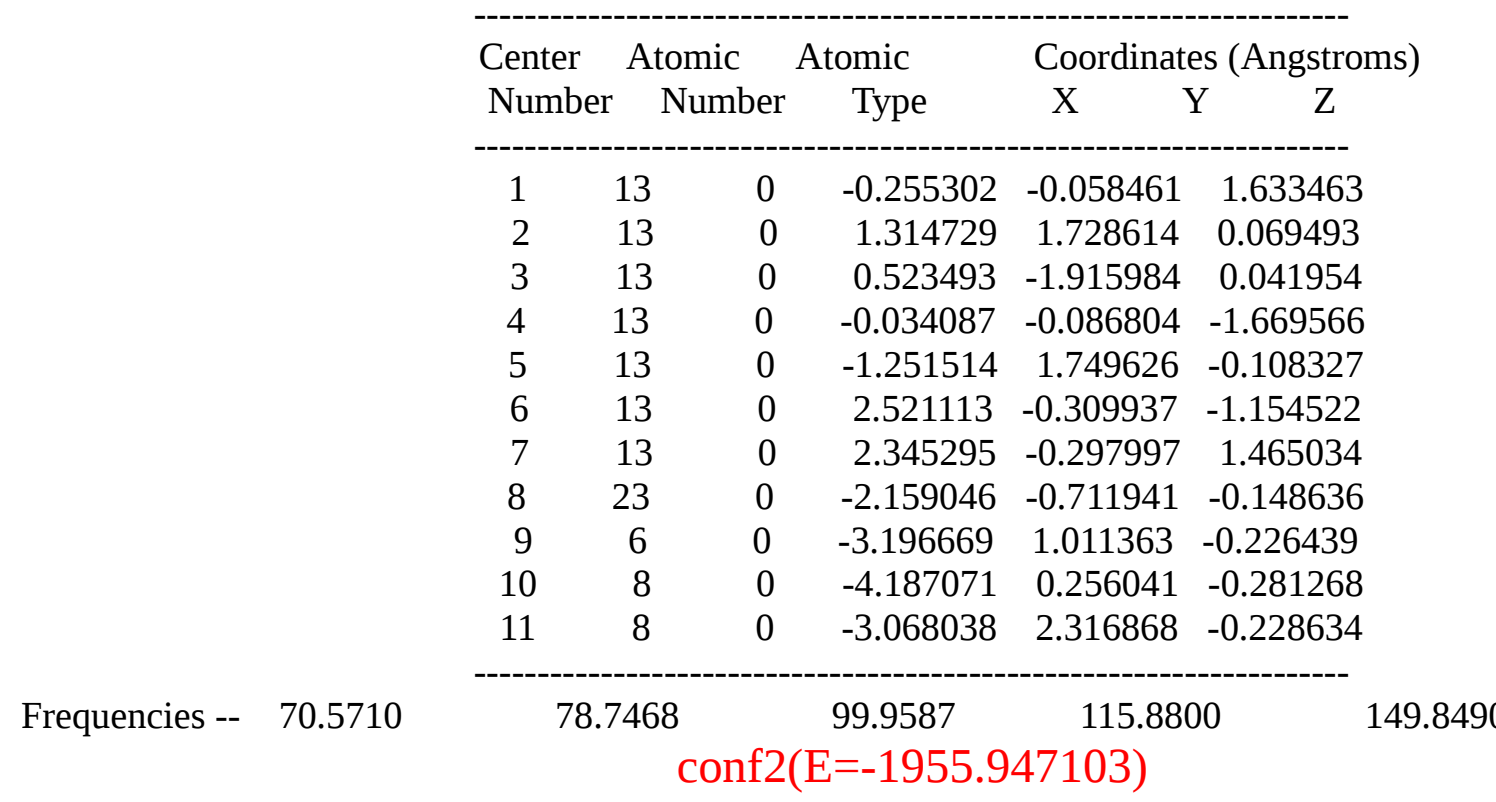

151.1123

\begin{tabular}{|c|c|c|c|c|c|}
\hline \multirow{2}{*}{$\begin{array}{l}\text { Center } \\
\text { Number }\end{array}$} & \multicolumn{2}{|c|}{ Atomic } & Atomic & \multicolumn{2}{|c|}{ Coordinates (Angstrom } \\
\hline & & Number & Type & $\mathrm{X} \quad \mathrm{Y}$ & $Y \quad Z$ \\
\hline 1 & 13 & 0 & -0.335162 & 0.275304 & 1.558555 \\
\hline 2 & 13 & 0 & 1.398659 & 1.920947 & 0.045521 \\
\hline 3 & 13 & 0 & 0.366182 & -1.783753 & 0.062132 \\
\hline 4 & 13 & 0 & -0.122710 & 0.199263 & -1.613085 \\
\hline 5 & 13 & 0 & -1.165763 & 2.199688 & -0.136163 \\
\hline 6 & 13 & 0 & 2.357733 & -0.289154 & -1.199218 \\
\hline 7 & 13 & 0 & 2.176787 & -0.229653 & 1.506258 \\
\hline 8 & 23 & 0 & -2.096253 & -0.649192 & -0.130572 \\
\hline 9 & 6 & 0 & -1.604210 & -2.565028 & -0.053149 \\
\hline 10 & 8 & 0 & -0.653638 & -3.436418 & 0.031150 \\
\hline 11 & 8 & 0 & -2.857229 & -2.637376 & -0.136234 \\
\hline
\end{tabular}

Frequencies -- 75.1353

$\begin{array}{lll}82.9195 & 87.6757 & 116.2476\end{array}$

138.8535

148.1455

conf3(E=-1955.963339)

\begin{tabular}{|c|c|c|c|c|c|}
\hline \multirow{2}{*}{$\begin{array}{l}\text { Center } \\
\text { Number }\end{array}$} & \multicolumn{2}{|c|}{ Atomic } & \multirow{2}{*}{$\begin{array}{l}\text { Atomic } \\
\text { Type }\end{array}$} & \multicolumn{2}{|c|}{ Coordinates (Angstror } \\
\hline & & Number & & $\mathrm{X}$ & $\mathrm{Y} \quad \mathrm{Z}$ \\
\hline 1 & 13 & 0 & -0.533755 & -0.116852 & 1.992044 \\
\hline 2 & 13 & 0 & 2.051444 & 1.896134 & -0.983088 \\
\hline 3 & 13 & 0 & 0.298211 & -1.536016 & -0.151399 \\
\hline 4 & 13 & 0 & 0.051782 & 0.195057 & -2.029891 \\
\hline 5 & 13 & 0 & -0.364712 & 1.703615 & 0.004166 \\
\hline 6 & 13 & 0 & 2.577037 & -0.673306 & -1.105173 \\
\hline 7 & 13 & 0 & 1.862555 & 0.410395 & 1.223653 \\
\hline 8 & 23 & 0 & -2.108863 & -0.338585 & -0.094760 \\
\hline 9 & 6 & 0 & -2.571830 & -0.527043 & 1.854741 \\
\hline
\end{tabular}




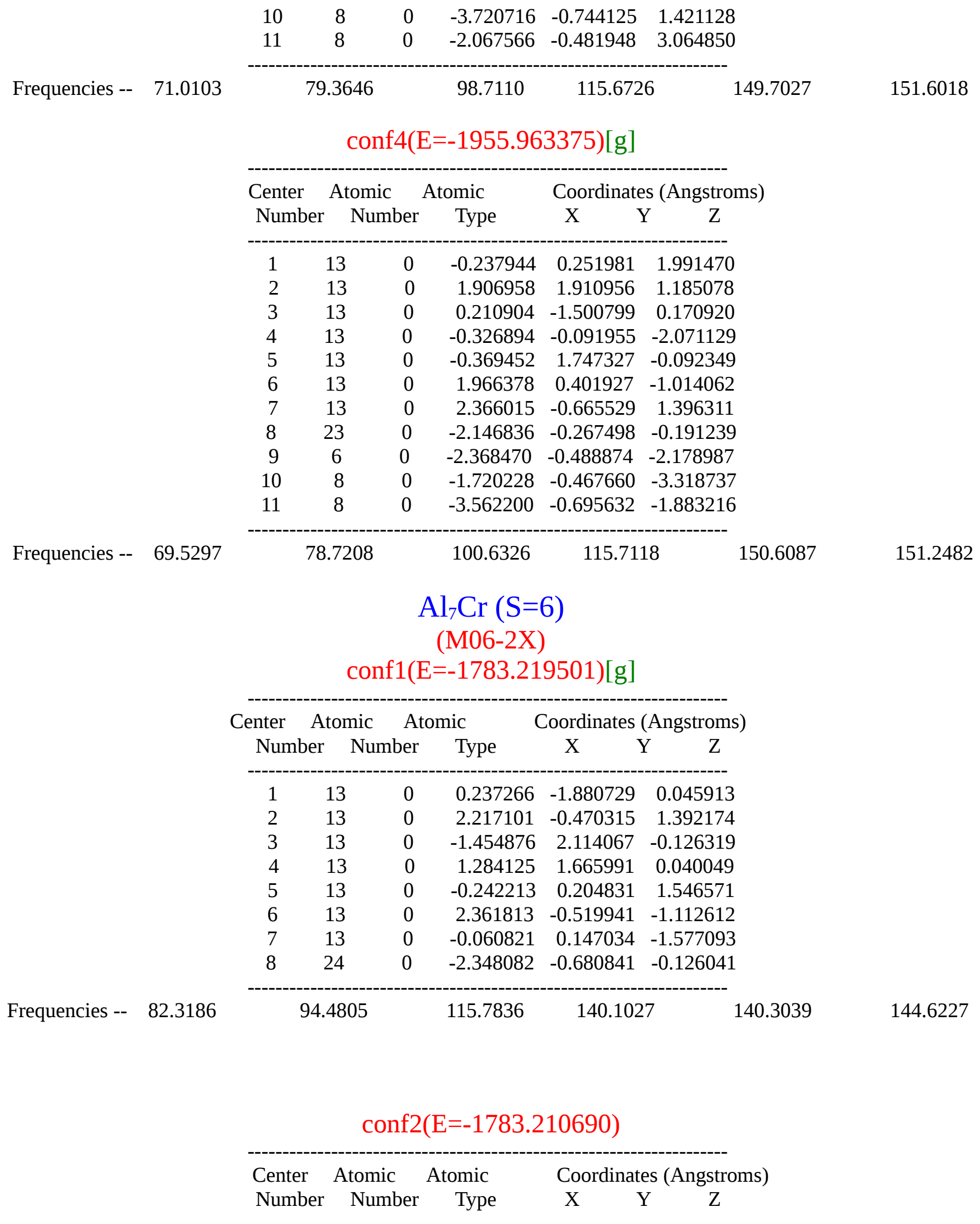




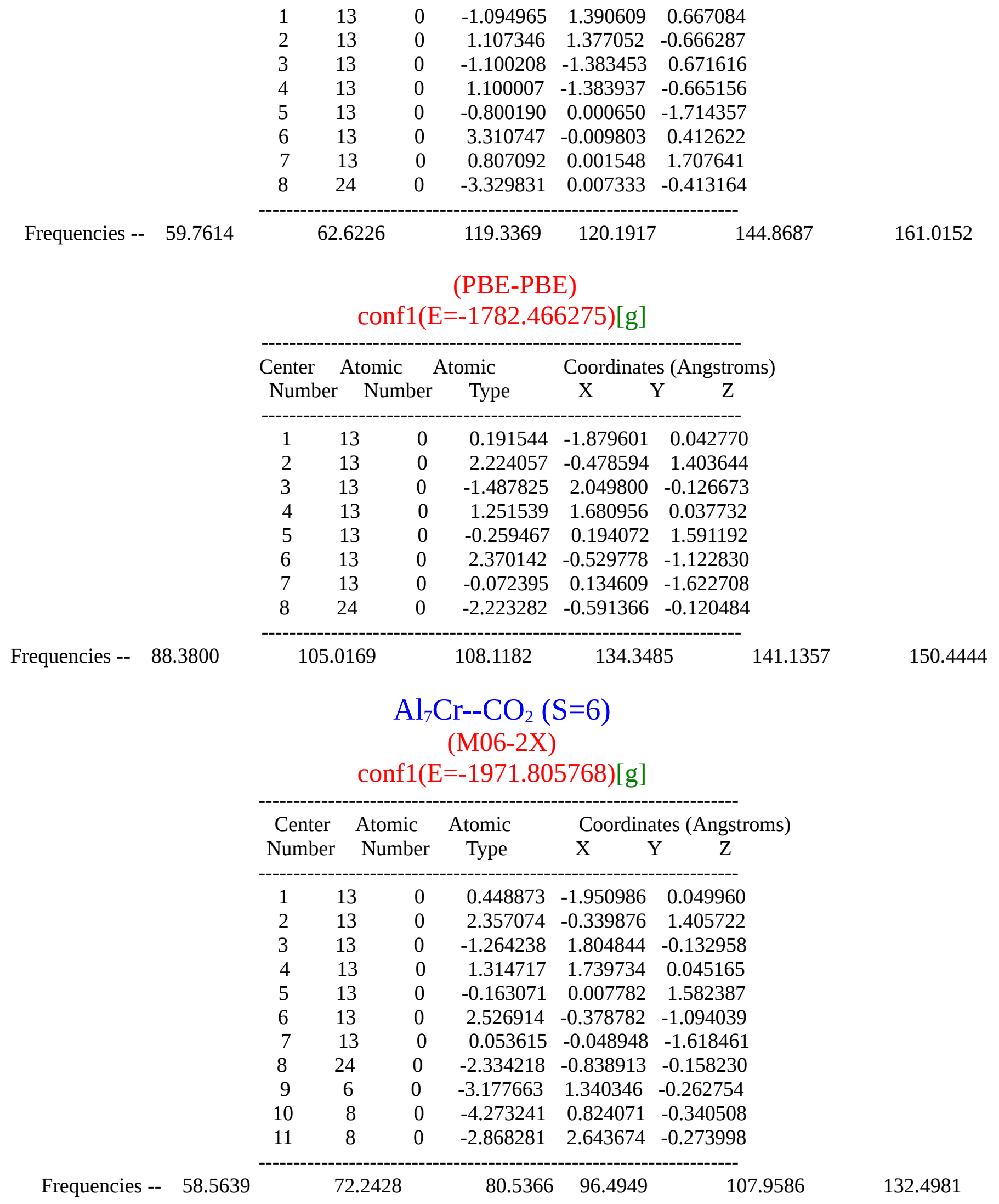




\section{conf2(E=-1971.799444)}

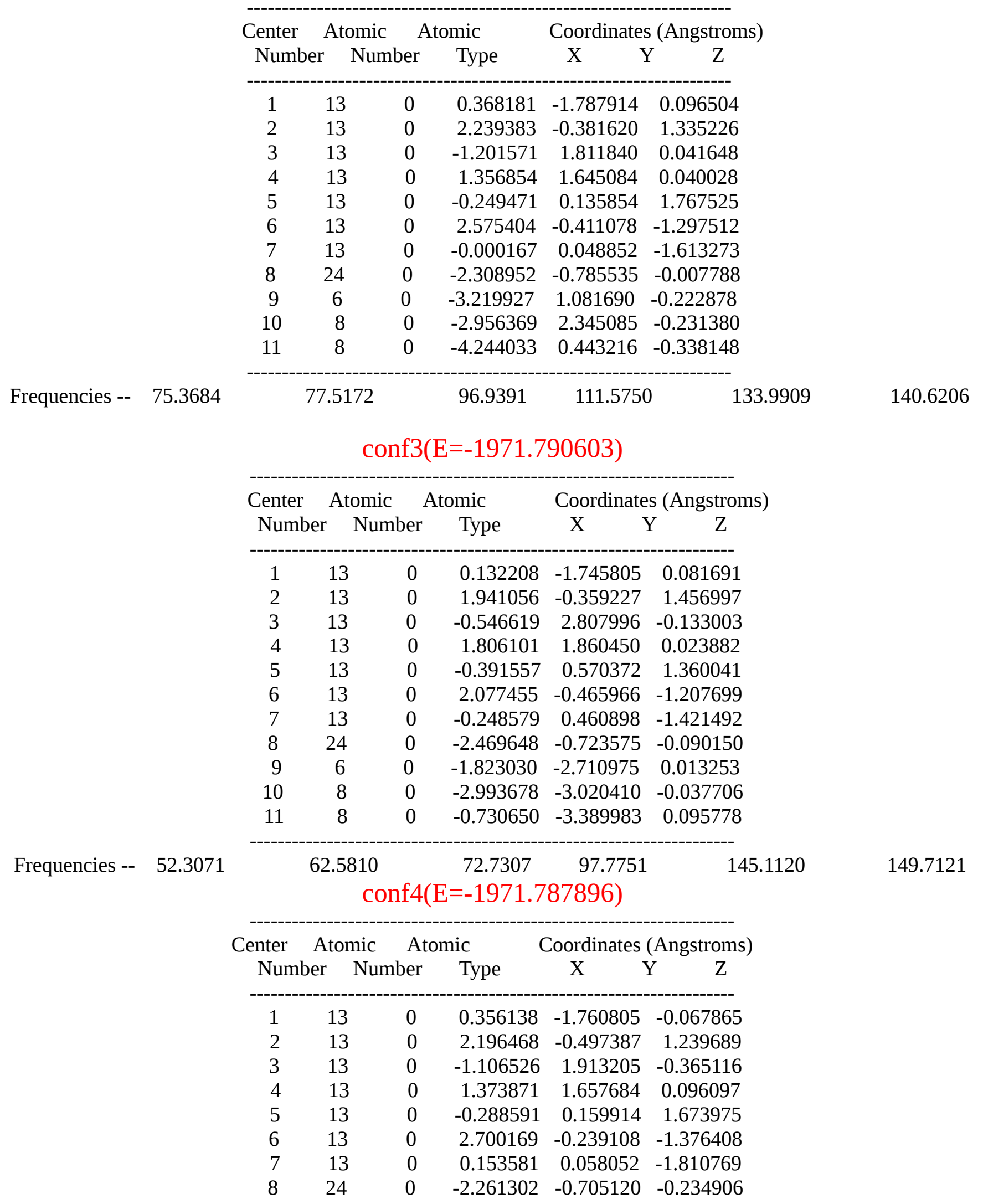




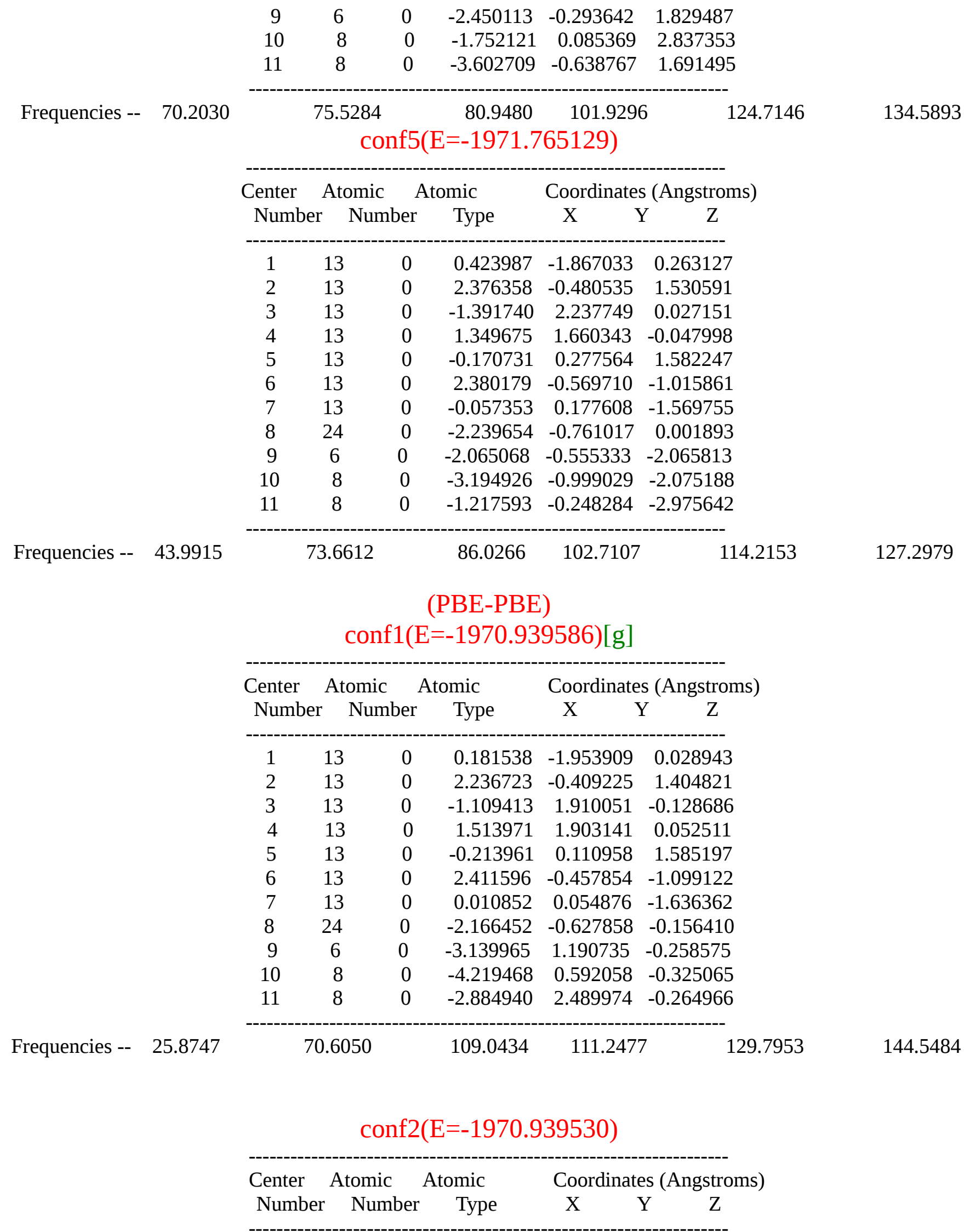




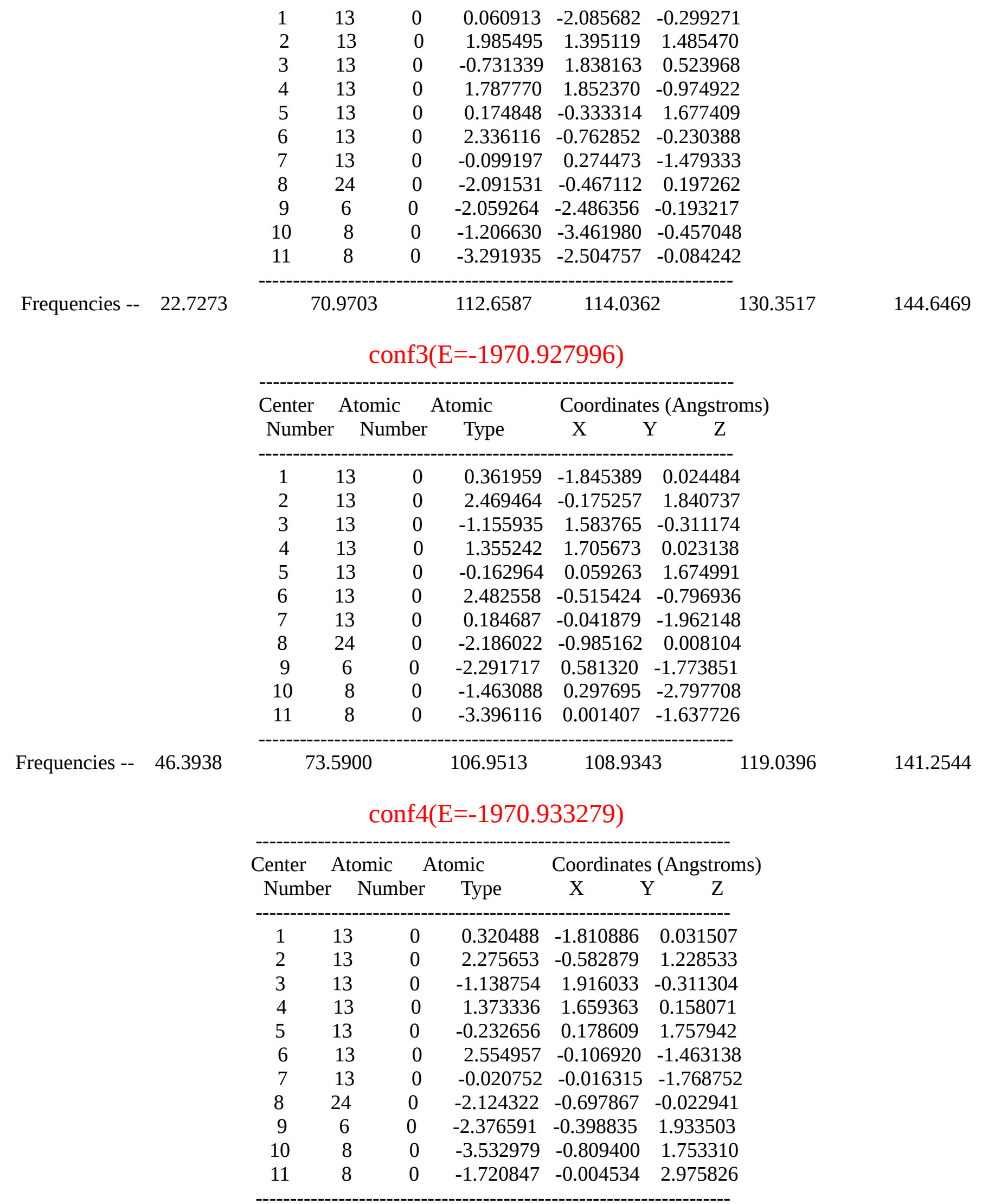




\begin{tabular}{|c|c|c|c|c|c|c|}
\hline & & A & $\begin{array}{r}\mathrm{Al}_{7} \mathrm{Mn}(\mathrm{S}= \\
(\mathrm{M} 06-2 \mathrm{X}) \\
\mathrm{E}=-1800.83\end{array}$ & $\begin{array}{l}\text { 5) } \\
6752)[g]\end{array}$ & & \\
\hline & Center & Atomic & Atomic & Coordinates & s (Angstroms) & \\
\hline & Number & Number & Type & $\begin{array}{ll}X & Y\end{array}$ & $\mathrm{Z}$ & \\
\hline & 1 & 13 & -0.111273 & 0.192809 & 1.665904 & \\
\hline & 2 & 13 & 1.092509 & 1.654249 & -0.016545 & \\
\hline & 3 & 13 & 0.327197 & -1.740954 & 0.006090 & \\
\hline & 4 & 13 & -0.229893 & 0.217191 & -1.704641 & \\
\hline & 5 & 13 & -1.607633 & 1.906819 & -0.013246 & \\
\hline & 6 & 13 & 2.248460 & -0.395081 & -1.235747 & \\
\hline & 7 & 13 & 2.471193 & -0.361958 & 1.368017 & \\
\hline & 8 & 25 & -2.218095 & -0.713801 & -0.071641 & \\
\hline Frequencies -- 84.5199 & 100 & 0.1662 & 108.9440 & 122.0492 & 133.6144 & 141.7320 \\
\hline & & conf2 & $(E=-1800.8$ & 28814) & & \\
\hline & Center & Atomic & Atomic & Coordinate & es (Angstroms) & \\
\hline & Number & Number & Type & $\begin{array}{ll}\mathrm{X} & \mathrm{Y}\end{array}$ & $\mathrm{Z}$ & \\
\hline & 1 & 13 & -0.896381 & 1.438252 & 0.721168 & \\
\hline & 2 & 13 & 1.149001 & 1.328673 & -0.829041 & \\
\hline & 3 & 13 & -1.167412 & -1.398732 & 0.714847 & \\
\hline & 4 & 13 & 0.996551 & -1.398814 & -0.685559 & \\
\hline & 5 & 13 & -0.855449 & 0.036280 & -1.759633 & \\
\hline & 6 & 13 & 3.166495 & 0.208105 & 0.519354 & \\
\hline & 7 & 13 & 0.823962 & -0.167557 & 1.750796 & \\
\hline & 8 & 25 & -3.216767 & -0.046206 & -0.431934 & \\
\hline Frequencies -- 53.1682 & & 102.7335 & 103.5987 & 129.767 & 162.5045 & 173.6734 \\
\hline & & & (PBE-PB & & & \\
\hline & & conf1(E & $E=-1800.07$ & 8332)[g] & & \\
\hline & Center & Atomic & Atomic & Coordinate & es (Angstroms) & \\
\hline & Number & Number & Type & $\mathrm{X} \quad \mathrm{Y}$ & & \\
\hline & 1 & 13 & -0.139191 & 0.141423 & 1.653628 & \\
\hline & 2 & 13 & 1.103692 & 1.721160 & -0.020385 & \\
\hline & 3 & 13 & 0.287075 & -1.804326 & -0.015083 & \\
\hline & 4 & 13 & -0.281796 & 0.171242 & -1.720926 & \\
\hline & 5 & 13 & -1.585588 & 1.889477 & -0.027066 & \\
\hline & 6 & 13 & 2.212600 & -0.365616 & -1.209642 & \\
\hline & 7 & 13 & 2.451924 & -0.350969 & 1.381474 & \\
\hline & 8 & 25 & -2.076251 & -0.643117 & -0.043810 & \\
\hline
\end{tabular}




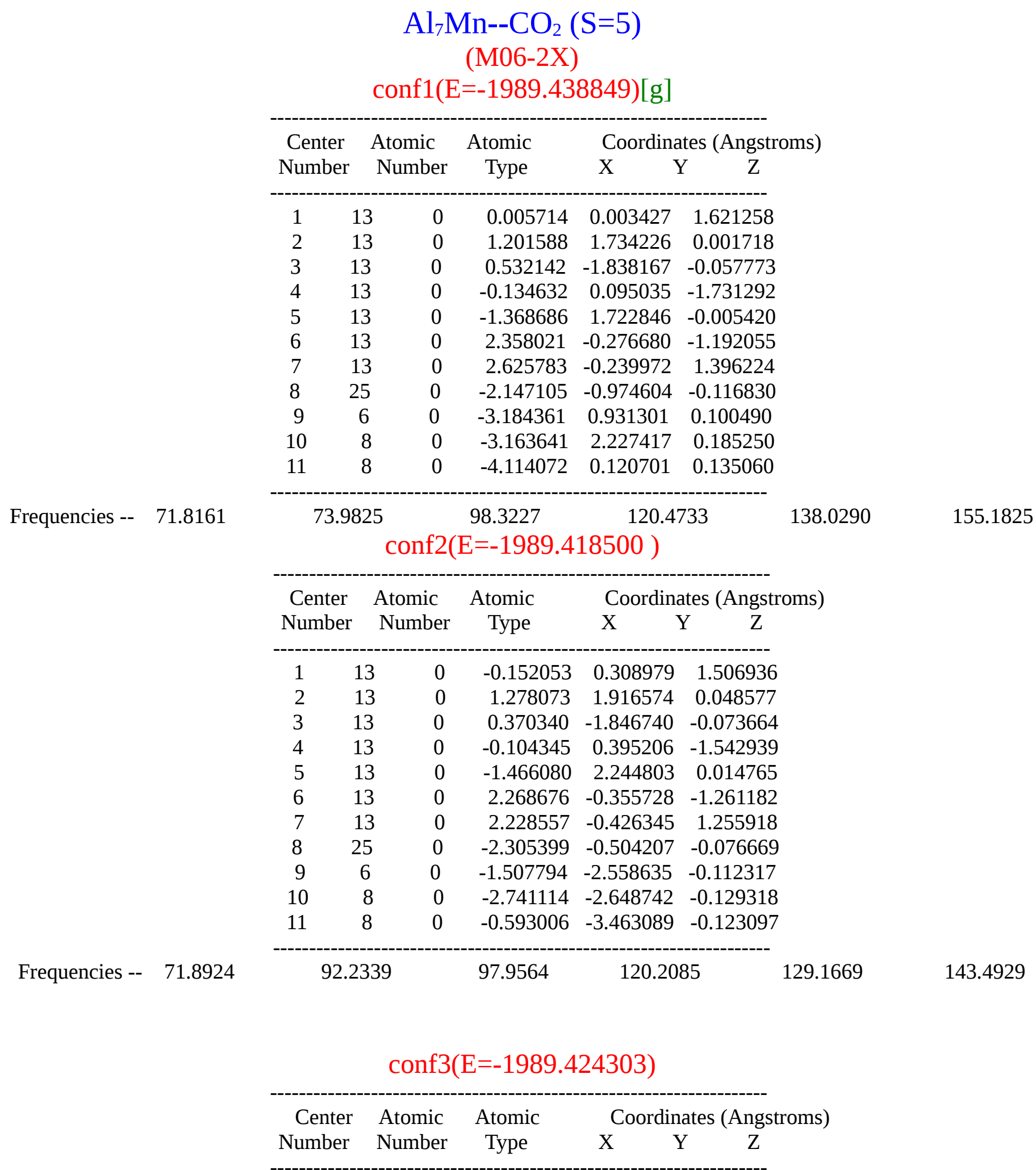




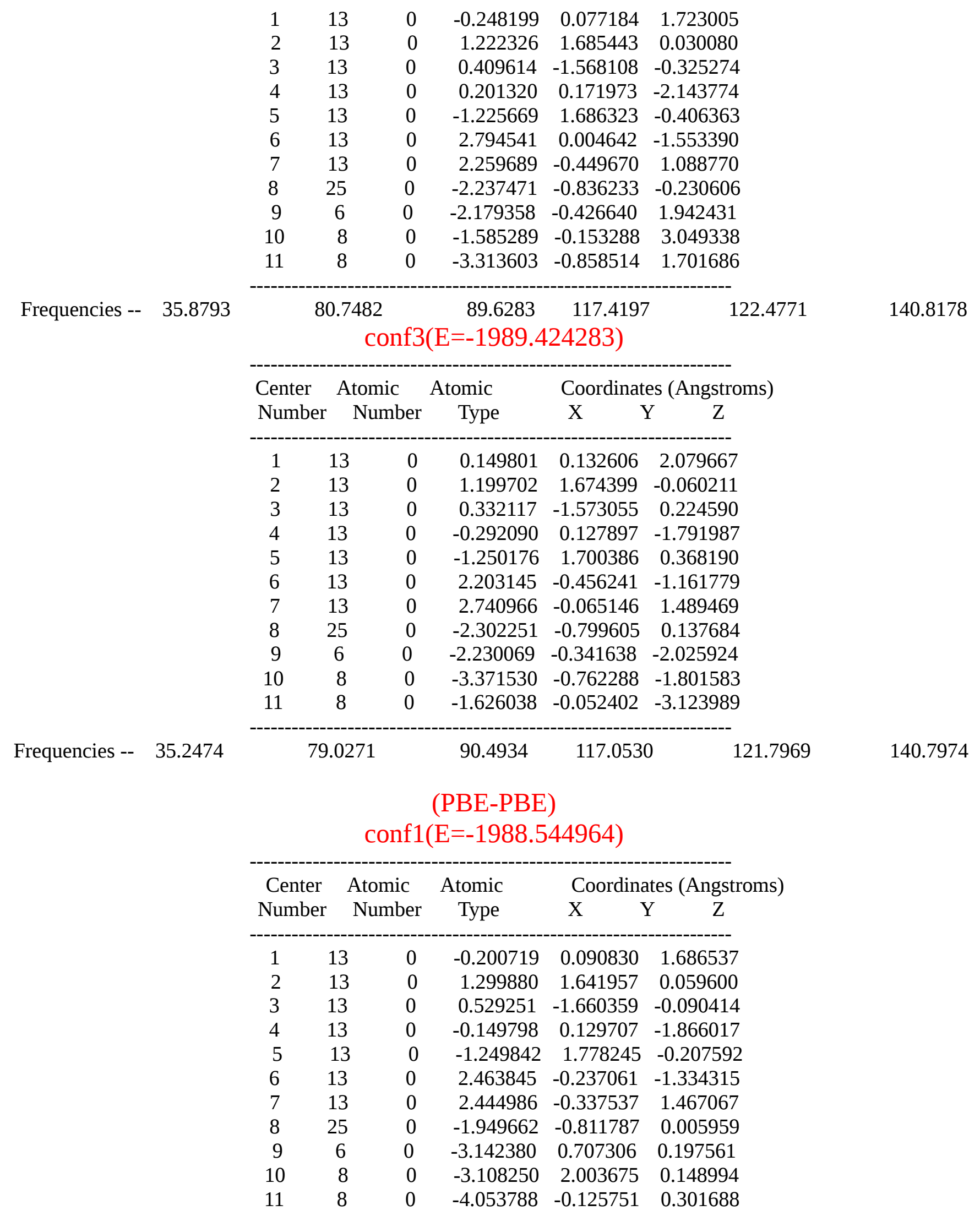




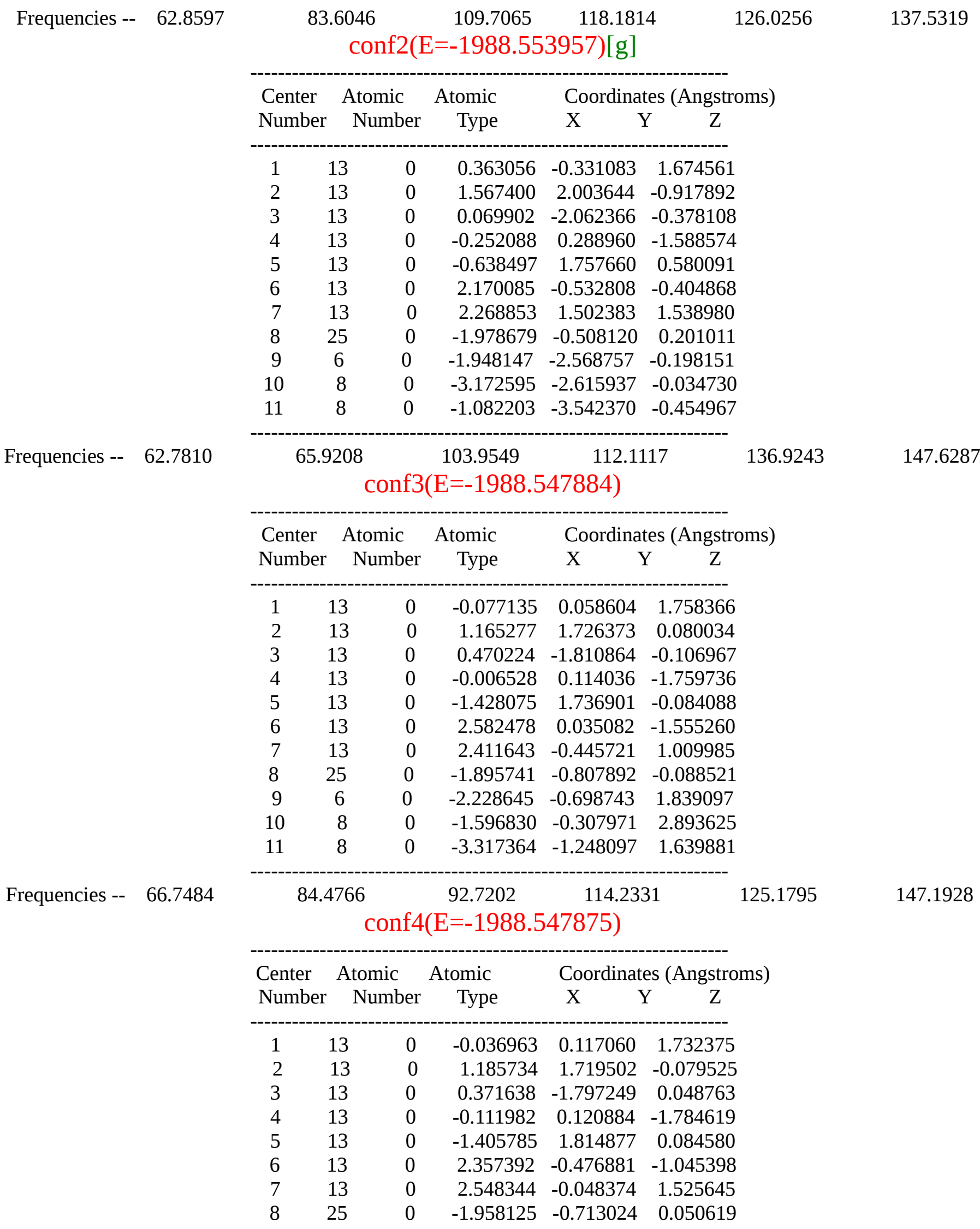




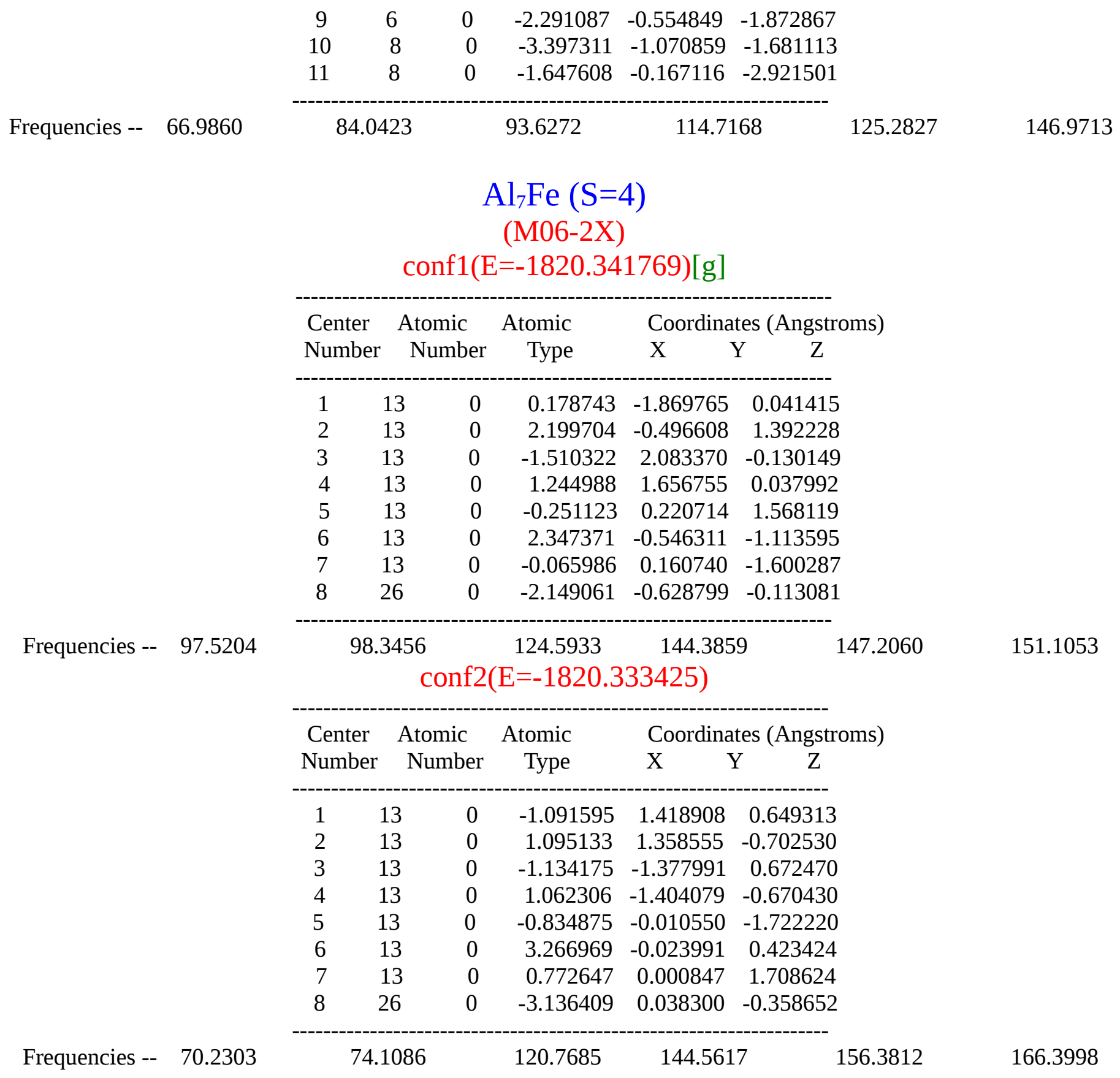

(PBE-PBE)

conf1(E=-1819.606717)[g] 


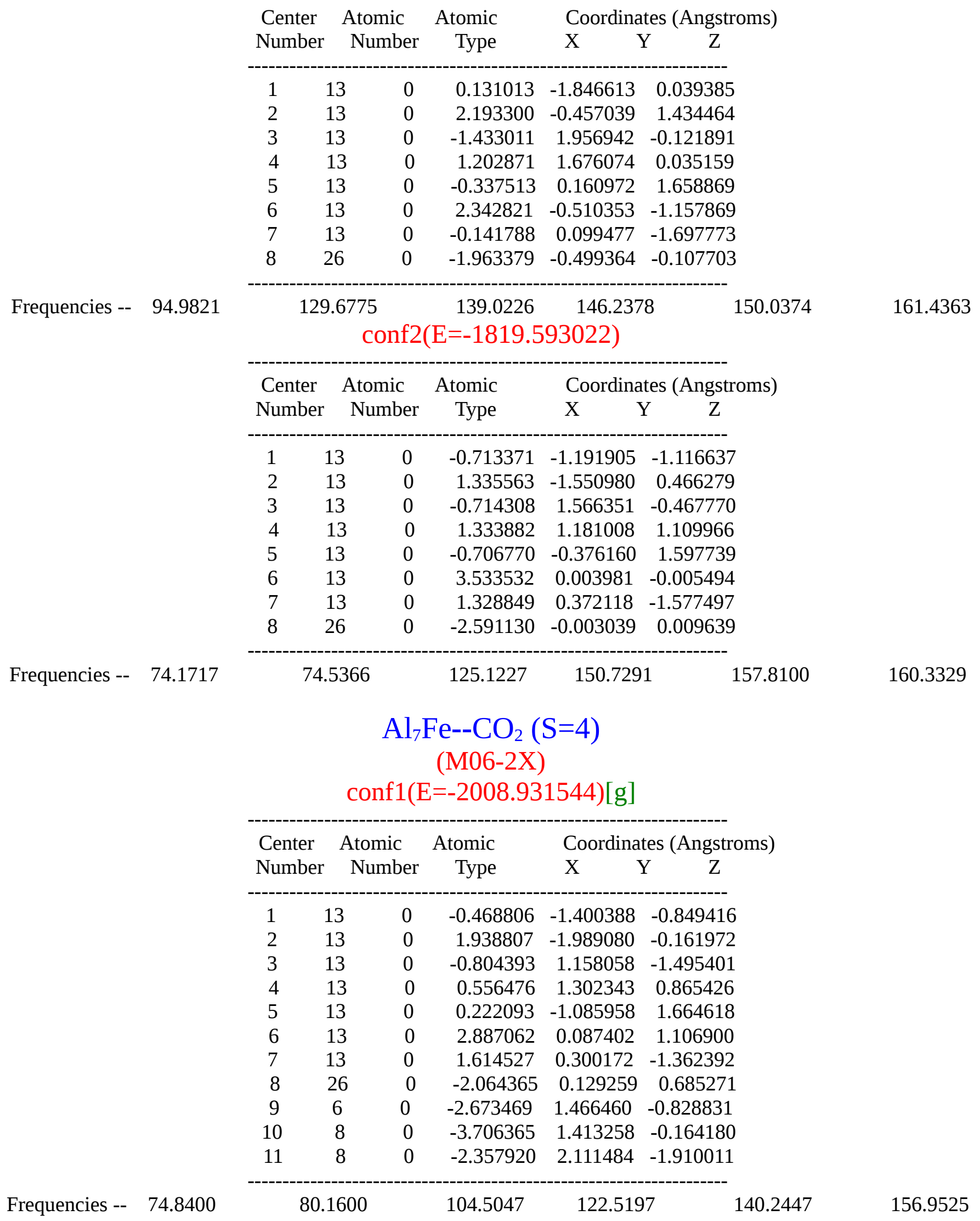




\section{conf2(E=-2008.912341)}

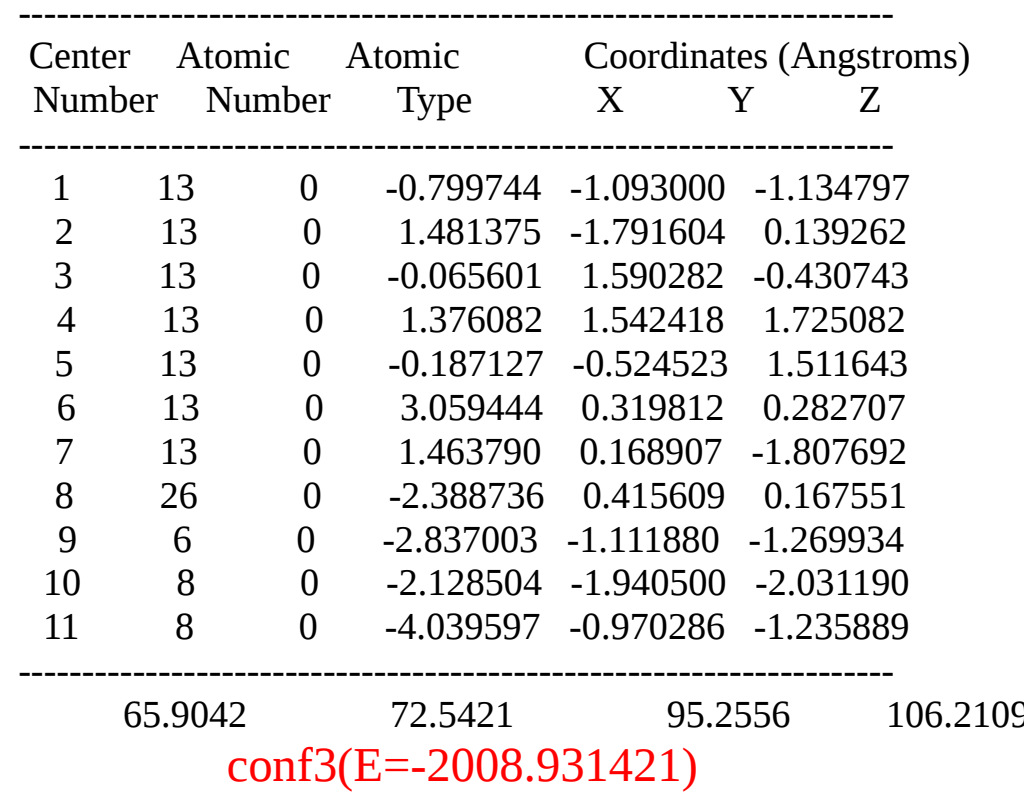

143.5851

Frequencies -- 51.3538

\begin{tabular}{rrrrrr}
\multicolumn{2}{c}{ Center } & \multicolumn{2}{c}{ Atomic } & Atomic & \multicolumn{2}{c}{ Coordinates } \\
Number & Number & Type & X & Y & Z \\
-1 & 13 & 0 & -0.501873 & -1.085047 & -1.173645 \\
2 & 13 & 0 & 1.672517 & -1.295864 & 0.486501 \\
3 & 13 & 0 & 0.114353 & 1.468976 & -1.225649 \\
4 & 13 & 0 & 0.580079 & 1.010832 & 1.228521 \\
5 & 13 & 0 & -0.699358 & -1.389752 & 1.463809 \\
6 & 13 & 0 & 2.847941 & 1.155106 & -0.118127 \\
7 & 13 & 0 & 1.855670 & -0.410337 & -1.956440 \\
8 & 26 & 0 & -2.077098 & 0.597890 & 0.217227 \\
9 & 6 & 0 & -2.575293 & -0.742657 & 1.769040 \\
10 & 8 & 0 & -3.633700 & -0.124256 & 1.671795 \\
11 & 8 & 0 & -2.195706 & -1.717316 & 2.536759
\end{tabular}

Frequencies -- 70.8400

$\begin{array}{ccc}80.1306 & 105.7528 & 124.1470 \\ (\mathrm{PBE}-\mathrm{PBE}) & \\ \operatorname{conf1}(\mathrm{E}=-2008.073465)\end{array}$

\begin{tabular}{|c|c|c|c|c|c|}
\hline \multirow{2}{*}{$\begin{array}{l}\text { Center } \\
\text { Number }\end{array}$} & \multirow{2}{*}{\multicolumn{2}{|c|}{$\begin{array}{l}\text { Atomic } \\
\text { Number }\end{array}$}} & \multirow{2}{*}{$\begin{array}{r}\text { Atomic } \\
\text { Type }\end{array}$} & \multicolumn{2}{|c|}{ Coordinates (Angstro } \\
\hline & & & & $\mathrm{X}$ & $Y \quad Z$ \\
\hline 1 & 13 & 0 & & -1.760949 & 0.182460 \\
\hline 2 & 13 & 0 & 89 & -0.352893 & 1.325770 \\
\hline 3 & 13 & 0 & -1.168254 & 1.845737 & 0.216034 \\
\hline 4 & 13 & 0 & 1.3679 & 1.606573 & -0.058956 \\
\hline 5 & 13 & 0 & -0.143491 & 0.180443 & 1.918755 \\
\hline 6 & 13 & 0 & 2.4949 & -0.523077 & -1.388051 \\
\hline 7 & 13 & 0 & -0.153167 & -0.000597 & -1.594028 \\
\hline
\end{tabular}




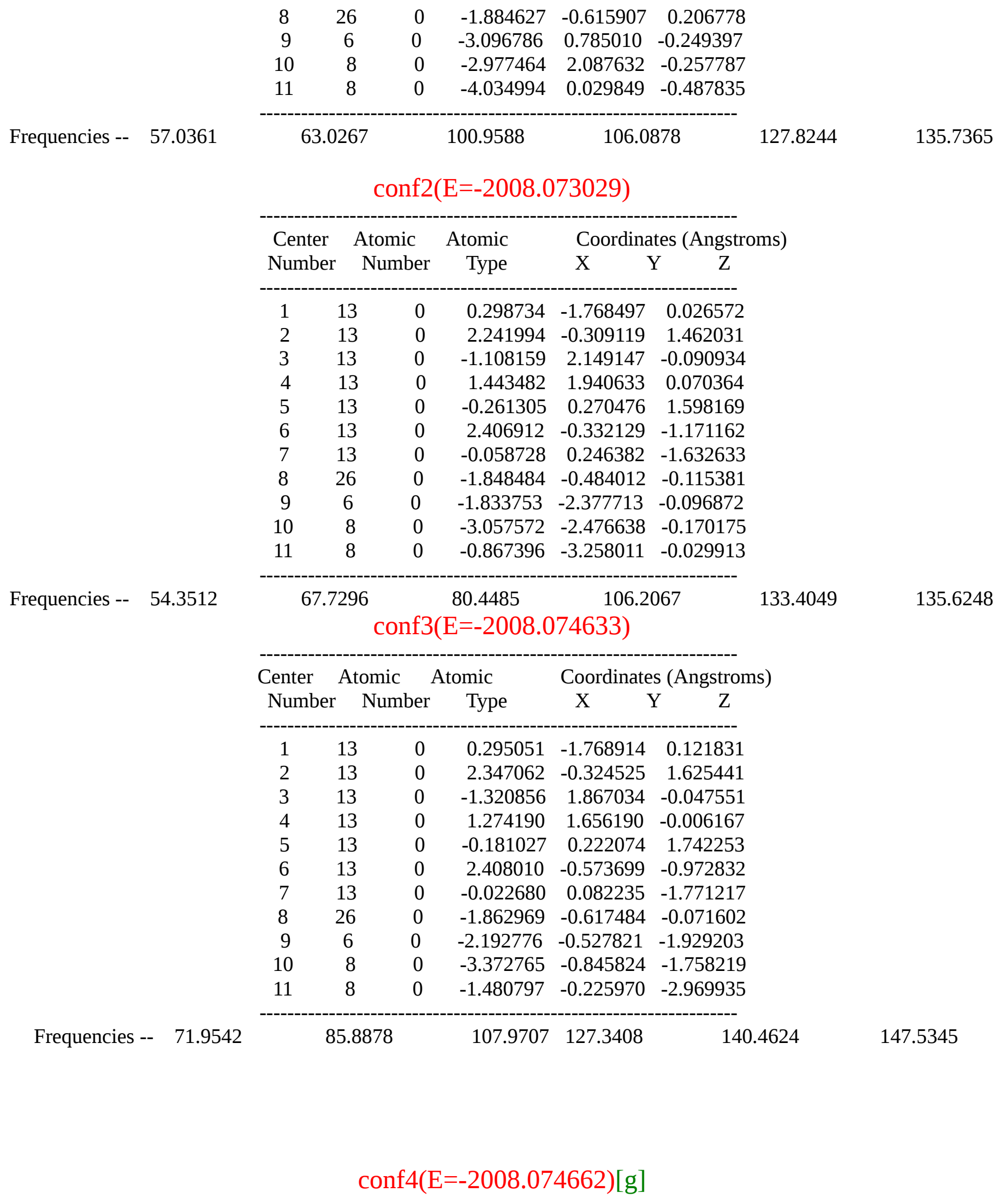




\begin{tabular}{|c|c|c|c|c|c|}
\hline \multirow{2}{*}{$\begin{array}{l}\text { Center } \\
\text { Numb }\end{array}$} & \multicolumn{2}{|c|}{ Atomic } & Atomic & \multicolumn{2}{|c|}{ Coordinates (Angstroms) } \\
\hline & & Number & Type & $\begin{array}{ll}X & Y\end{array}$ & $\begin{array}{ll}Y & Z\end{array}$ \\
\hline 1 & 13 & 0 & 0.474762 & -1.792902 & -0.118634 \\
\hline 2 & 13 & 0 & 2.403072 & -0.496561 & 1.181364 \\
\hline 3 & 13 & 0 & -1.303347 & 1.768571 & -0.171375 \\
\hline 4 & 13 & 0 & 1.287324 & 1.672861 & 0.069953 \\
\hline 5 & 13 & 0 & -0.125971 & 0.056603 & 1.704377 \\
\hline 6 & 13 & 0 & 2.614105 & -0.273446 & -1.412001 \\
\hline 7 & 13 & 0 & 0.093103 & 0.160925 & -1.807428 \\
\hline 8 & 26 & 0 & -1.740069 & -0.736080 & -0.174466 \\
\hline 9 & 6 & 0 & -2.274372 & -0.642997 & 1.634599 \\
\hline 10 & 8 & 0 & -1.692530 & -0.300252 & 2.741464 \\
\hline 11 & 8 & 0 & -3.413720 & -1.015685 & 1.342627 \\
\hline & +.0400 & & 107.8985 & 127.2273 & \\
\hline
\end{tabular}

146.7009

Frequencies -- 73.3671

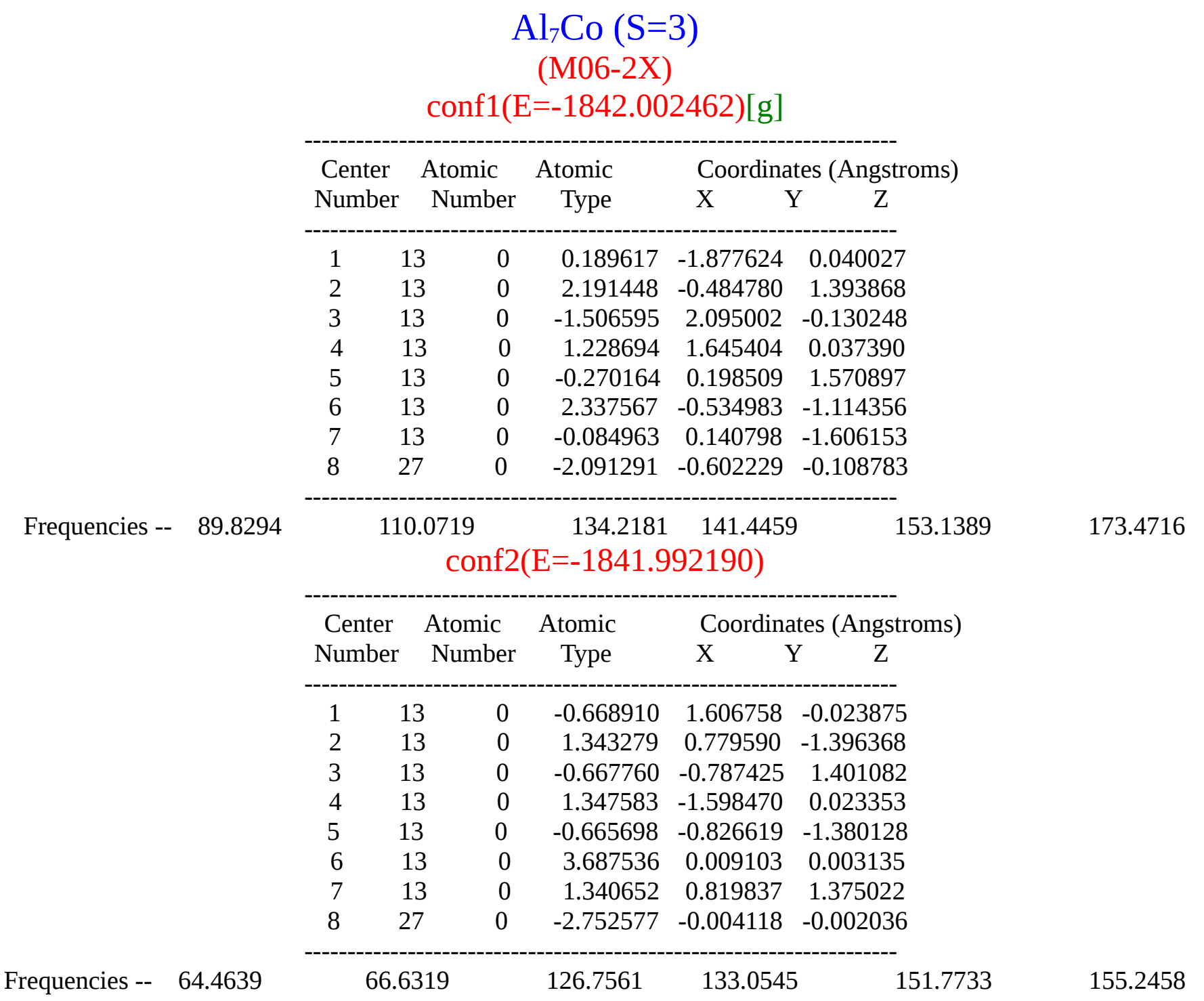




\section{conf3(E=-1841.991177)}

Center Atomic Atomic Coordinates (Angstroms)

Frequencies -- 68.0638

\begin{tabular}{rrrrrr} 
Number & Number & \multicolumn{1}{c}{ Type } & X & Y & $Z$ \\
- & 13 & 0 & -0.679586 & 1.598010 & -0.023403 \\
2 & 13 & 0 & 1.358943 & 0.784907 & -1.384842 \\
3 & 13 & 0 & -0.645520 & -0.777298 & 1.422077 \\
4 & 13 & 0 & 1.335336 & -1.608823 & 0.013425 \\
5 & 13 & 0 & -0.666596 & -0.804064 & -1.383993 \\
6 & 13 & 0 & 3.676759 & -0.014576 & 0.005024 \\
7 & 13 & 0 & 1.352438 & 0.835487 & 1.361381 \\
8 & 27 & 0 & -2.767668 & -0.014986 & -0.009485
\end{tabular}

\begin{tabular}{|c|c|c|c|c|c|c|c|}
\hline 71. & $\begin{array}{r}3453 \\
\text { C }\end{array}$ & conf1(1 & $\begin{array}{l}129.9601 \\
(\mathrm{PBE}-\mathrm{PBE} \\
=-1841.26\end{array}$ & $\begin{array}{l}\quad 136.502 \\
\text { Е) } \\
5601)[g]\end{array}$ & & 148.2233 & 161.4708 \\
\hline Center & & tomic & Atomic & Coordinat & tes (Angstro & ms) & \\
\hline Numbe & & Number & Type & $\begin{array}{ll}X & Y\end{array}$ & $\begin{array}{l}Y \\
Z\end{array}$ & & \\
\hline 1 & 13 & 0 & 0.110989 & -1.871018 & 0.038552 & & \\
\hline 2 & 13 & 0 & 2.174025 & -0.470782 & 1.419785 & & \\
\hline 3 & 13 & 0 & -1.476395 & 1.950991 & -0.120233 & & \\
\hline 4 & 13 & 0 & 1.213139 & 1.664340 & 0.037409 & & \\
\hline 5 & 13 & 0 & -0.346849 & 0.172854 & 1.637902 & & \\
\hline 6 & 13 & 0 & 2.325195 & -0.533128 & -1.148227 & & \\
\hline 7 & 13 & 0 & -0.146606 & 0.112846 & -1.678498 & & \\
\hline 8 & 27 & 0 & -1.859185 & -0.446006 & -0.104049 & & \\
\hline & & $\begin{array}{l}21 \\
\text { conf2 }\end{array}$ & $\begin{array}{l}148.0400 \\
E=-1841.2\end{array}$ & $\begin{array}{l}150.96 \\
41237)\end{array}$ & & 159.7718 & 166.4722 \\
\hline Center & & tomic & Atomic & Coordinate & tes (Angstro & ms) & \\
\hline Numbe & & Number & Type & $\begin{array}{ll}X & Y\end{array}$ & $\begin{array}{l}Y \\
Z\end{array}$ & & \\
\hline 1 & 13 & 0 & -0.657462 & 1.637701 & -0.122759 & & \\
\hline 2 & 13 & 0 & 1.372432 & 0.706359 & -1.461185 & & \\
\hline 3 & 13 & 0 & -0.653597 & -0.884884 & 1.385080 & & \\
\hline 4 & 13 & 0 & 1.374267 & -1.621844 & -0.072898 & & \\
\hline 5 & 13 & 0 & -0.772354 & -0.818130 & -1.368599 & & \\
\hline 6 & 13 & 0 & 3.490765 & 0.058555 & 0.096327 & & \\
\hline 7 & 13 & 0 & 1.278227 & 0.869971 & 1.453706 & & \\
\hline 8 & 27 & 0 & -2.468174 & 0.050926 & 0.090514 & & \\
\hline 72.2 & 940 & & 104.9073 & 143.10 & 097 & 159.1262 & 168.6381 \\
\hline
\end{tabular}

Frequencies -- 59.1716

$$
\mathrm{Al}_{7} \mathrm{Co}--\mathrm{CO}_{2}(\mathrm{~S}=3)
$$

(M06-2X) 


\section{$\operatorname{conf1(E=-2030.588766)[g]~}$}

\begin{tabular}{|c|c|c|c|c|c|}
\hline \multirow{2}{*}{$\begin{array}{l}\text { Center } \\
\text { Number }\end{array}$} & \multirow{2}{*}{\multicolumn{2}{|c|}{$\begin{array}{l}\text { Atomic } \\
\text { Number }\end{array}$}} & \multirow{2}{*}{$\begin{array}{l}\text { Atomic } \\
\text { Type }\end{array}$} & \multicolumn{2}{|c|}{ Coordinates (Angstro } \\
\hline & & & & $\mathrm{X}$ & $\mathrm{Y} \quad \mathrm{Z}$ \\
\hline 1 & 13 & 0 & 0.461689 & -1.949096 & 0.058072 \\
\hline 2 & 13 & 0 & & -0.3 & 6468 \\
\hline 3 & 13 & 0 & -1.3 & 1.6 & -0.063212 \\
\hline 4 & 13 & 0 & & 1.732372 & 0.052823 \\
\hline 5 & 13 & 0 & -0.0978 & -0.016617 & 1.646182 \\
\hline 6 & 13 & 0 & 2.501902 & -0.346554 & -1.124493 \\
\hline 7 & 13 & 0 & 0.033341 & -0.057668 & -1.613363 \\
\hline 8 & 27 & 0 & -2.071948 & -0.747122 & -0.049020 \\
\hline 9 & 6 & 0 & -3.326196 & 1.008920 & -0.165293 \\
\hline 10 & 8 & 0 & -2.973573 & 2.329458 & -0.168539 \\
\hline 11 & 8 & 0 & -4.461267 & 0.613431 & -0.201998 \\
\hline
\end{tabular}

Frequencies -- 50.1802

$\begin{array}{ccc}68.6588 & 92.6390 & 108.4540\end{array}$

139.7502

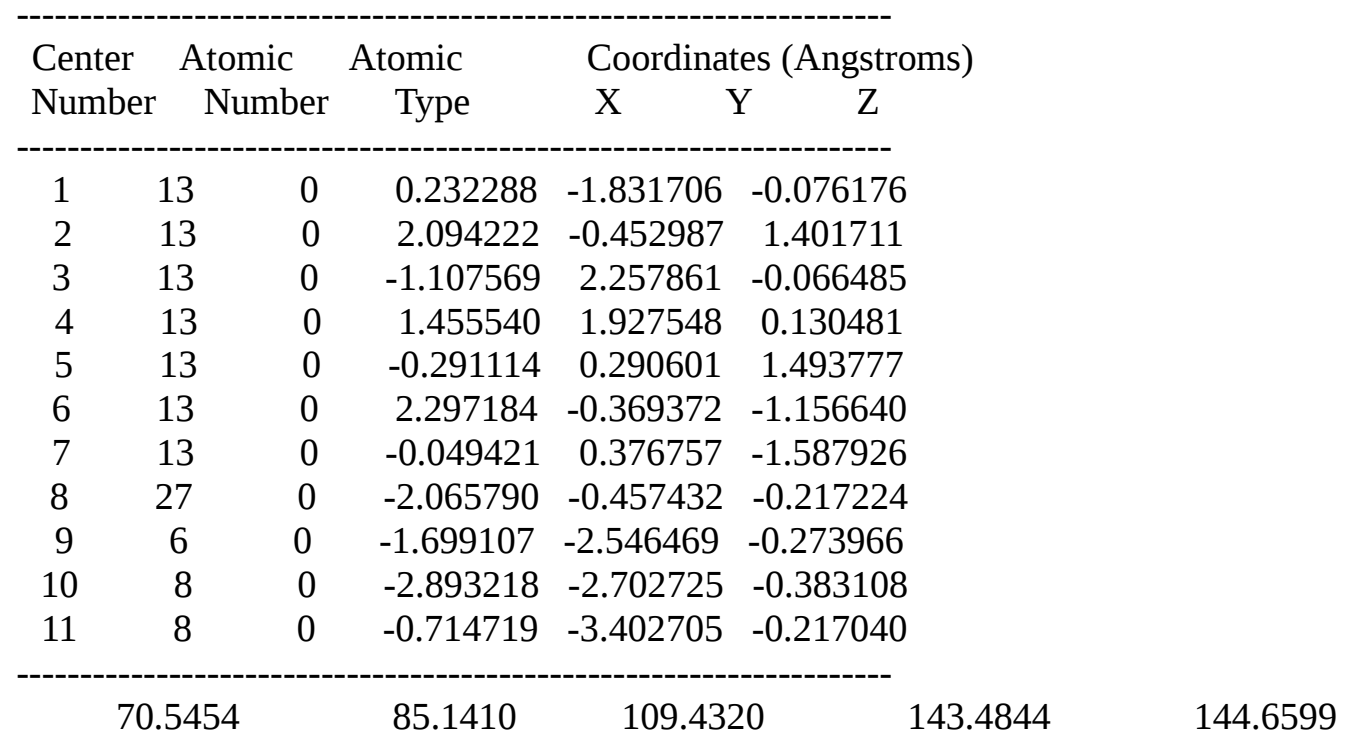

Frequencies -- 66.9254

$$
\text { conf3(E=-2030.542782) }
$$

\begin{tabular}{|c|c|c|c|c|c|}
\hline \multirow{2}{*}{$\begin{array}{l}\text { Center } \\
\text { Number }\end{array}$} & \multirow{2}{*}{\multicolumn{2}{|c|}{$\begin{array}{l}\text { Atomic } \\
\text { Number }\end{array}$}} & \multirow{2}{*}{$\begin{array}{l}\text { Atomic } \\
\text { Type }\end{array}$} & \multicolumn{2}{|c|}{ Coordinates (Angstro } \\
\hline & & & & $\mathrm{X}$ & $\mathrm{Y} \quad \mathrm{Z}$ \\
\hline 1 & 13 & 0 & & -1.730500 & 0.100888 \\
\hline 2 & 13 & 0 & 2.0 & -0.44 & 1.362158 \\
\hline 3 & 13 & 0 & -1 & 2.0 & -0.330326 \\
\hline 4 & 13 & 0 & 1.3291 & 1.647433 & 0.032795 \\
\hline 5 & 13 & 0 & -0.3643 & 0.345342 & 1.735819 \\
\hline 6 & 13 & 0 & 2.4 & -0.525169 & -1.268051 \\
\hline 7 & 13 & 0 & 0.003132 & 0.088018 & -1.724391 \\
\hline 8 & 27 & 0 & -2.071653 & -0.484536 & -0.043998 \\
\hline
\end{tabular}




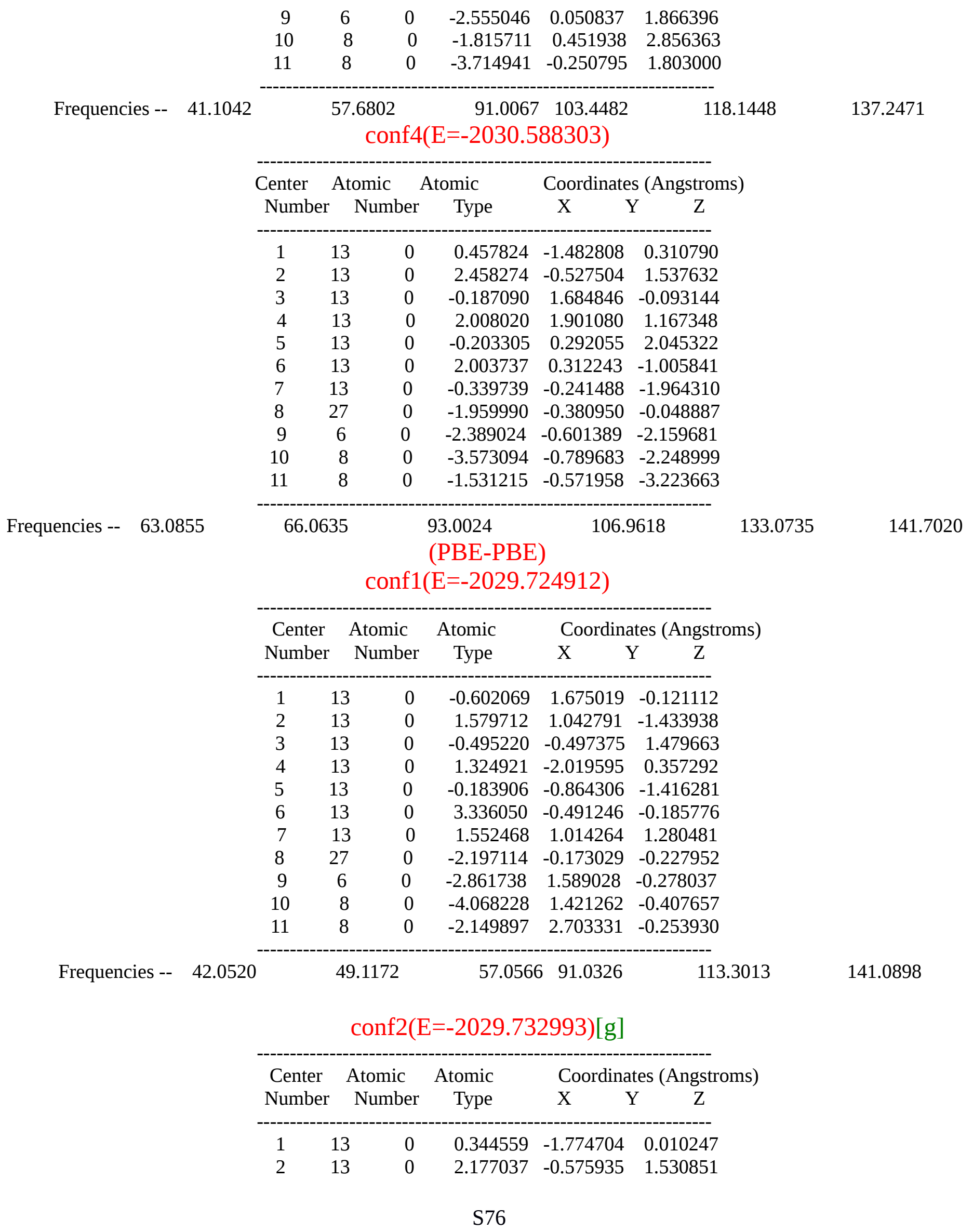




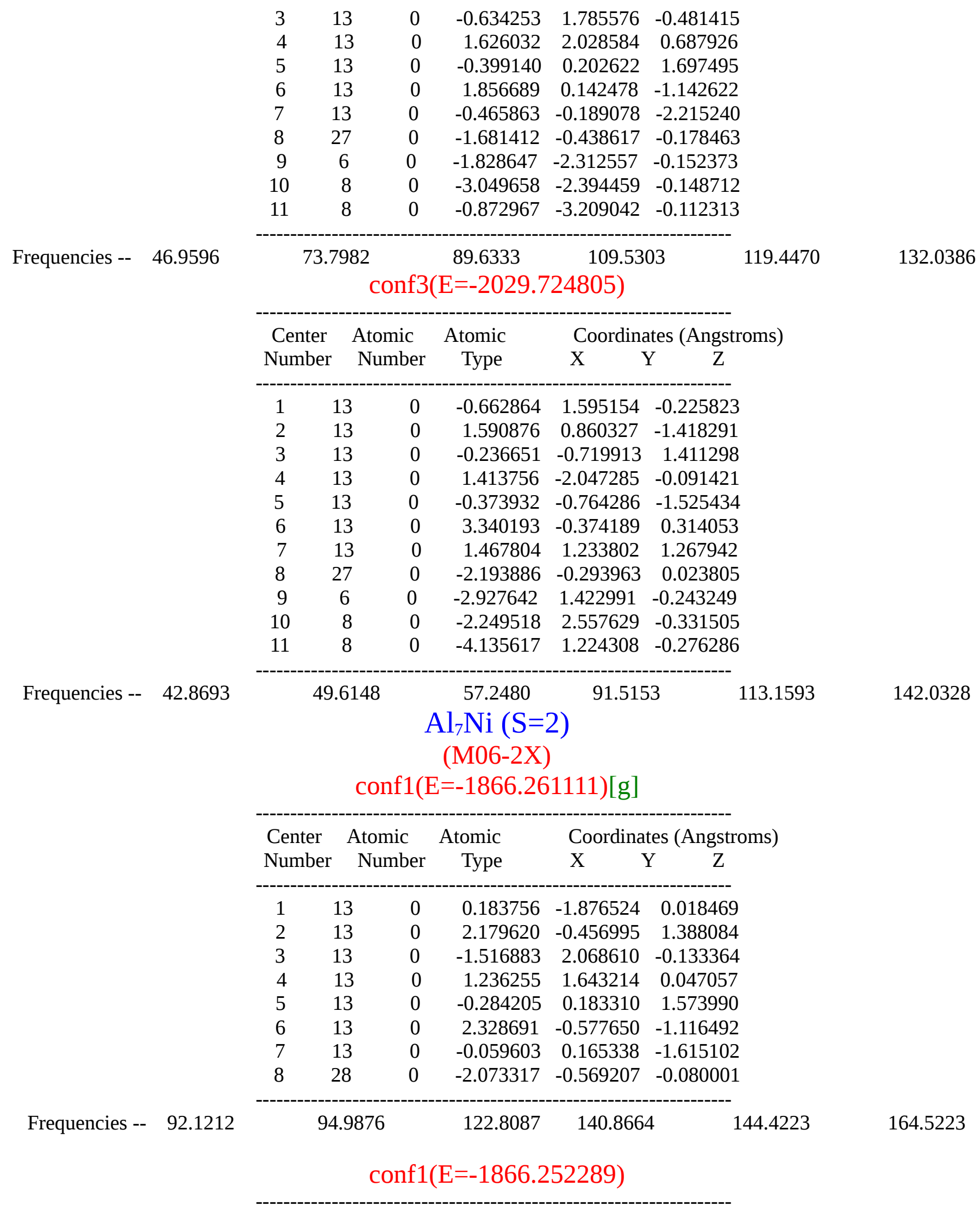




\begin{tabular}{|c|c|c|c|c|c|}
\hline \multirow{2}{*}{$\begin{array}{l}\text { Center } \\
\text { Numb }\end{array}$} & \multicolumn{2}{|c|}{ Atomic } & Atomic & \multicolumn{2}{|c|}{ Coordinates (Angstroms) } \\
\hline & & Number & Type & $\mathrm{X}$ & Y $\quad Z$ \\
\hline 1 & 13 & 0 & -1.128697 & 1.394789 & 0.668065 \\
\hline 2 & 13 & 0 & 1.061336 & 1.385734 & -0.679370 \\
\hline 3 & 13 & 0 & -1.122566 & -1.400045 & 0.667699 \\
\hline 4 & 13 & 0 & 1.066551 & -1.381282 & -0.680748 \\
\hline 5 & 13 & 0 & -0.843263 & -0.000995 & -1.710671 \\
\hline 6 & 13 & 0 & 3.283553 & 0.005712 & 0.415718 \\
\hline 7 & 13 & 0 & 0.766890 & 0.000979 & 1.704575 \\
\hline 8 & 28 & 0 & -3.083803 & -0.004893 & -0.385270 \\
\hline
\end{tabular}

Frequencies -- 68.4249

\begin{tabular}{|c|c|c|c|c|c|c|c|}
\hline 71. & 946 & conf1(E & $\begin{aligned} & 126.7303 \\
&(\mathrm{PBE}-\mathrm{PBE} \\
&=-1865.52\end{aligned}$ & $\begin{array}{r}134.23 \\
3802)[g]\end{array}$ & & 153.3704 & 158.8912 \\
\hline Center & & Atomic & Atomic & Coordina & tes (Angstr & oms) & \\
\hline Numbel & & Number & Type & $\mathrm{X}$ & $Y \quad Z$ & & \\
\hline 1 & 13 & 0 & 0.127309 & -1.872778 & 0.030172 & & \\
\hline 2 & 13 & 0 & 2.107273 & -0.434633 & 1.438299 & & \\
\hline 3 & 13 & 0 & -1.386100 & 1.938103 & -0.116215 & & \\
\hline 4 & 13 & 0 & 1.245798 & 1.669725 & 0.046782 & & \\
\hline 5 & 13 & 0 & -0.387329 & 0.128635 & 1.675153 & & \\
\hline 6 & 13 & 0 & 2.268137 & -0.485950 & -1.157508 & & \\
\hline 7 & 13 & 0 & -0.171698 & 0.085260 & -1.721909 & & \\
\hline 8 & 28 & 0 & -1.809076 & -0.448265 & -0.112133 & & \\
\hline 13 & & & 141.4590 & 149.53 & & 153.8446 & 166.7638 \\
\hline
\end{tabular}

Frequencies -- 50.7909

\begin{tabular}{|c|c|c|c|c|c|}
\hline & & $\begin{array}{r}\text { Al } \\
\text { conf1( }\end{array}$ & $\begin{array}{r}{ }_{7} \mathrm{Ni}--\mathrm{CO}_{2} \\
(\mathrm{M} 06-2 \mathrm{X}) \\
=-2054.84\end{array}$ & $\begin{array}{l}(S=2) \\
9745)[g]\end{array}$ & \\
\hline Center & & Atomic & Atomic & Coordina & tes (Angstroms) \\
\hline Number & & Number & Type & $\mathrm{X} \quad \mathrm{Y}$ & $\mathrm{Y} \quad \mathrm{Z}$ \\
\hline 1 & 13 & 0 & 0.531210 & -1.955962 & 0.066210 \\
\hline 2 & 13 & 0 & 2.416255 & -0.312891 & 1.389320 \\
\hline 3 & 13 & 0 & -1.301430 & 1.679067 & -0.093576 \\
\hline 4 & 13 & 0 & 1.274787 & 1.713019 & 0.042223 \\
\hline 5 & 13 & 0 & -0.103910 & -0.040888 & 1.631307 \\
\hline 6 & 13 & 0 & 2.543027 & -0.347230 & -1.121001 \\
\hline 7 & 13 & 0 & 0.059069 & -0.100783 & -1.621439 \\
\hline 8 & 28 & 0 & -1.997681 & -0.727300 & -0.081250 \\
\hline 9 & 6 & 0 & -3.281488 & 0.994201 & -0.188986 \\
\hline 10 & 8 & 0 & -2.935945 & 2.321487 & -0.196950 \\
\hline 11 & 8 & 0 & -4.413693 & 0.598703 & -0.240924 \\
\hline
\end{tabular}




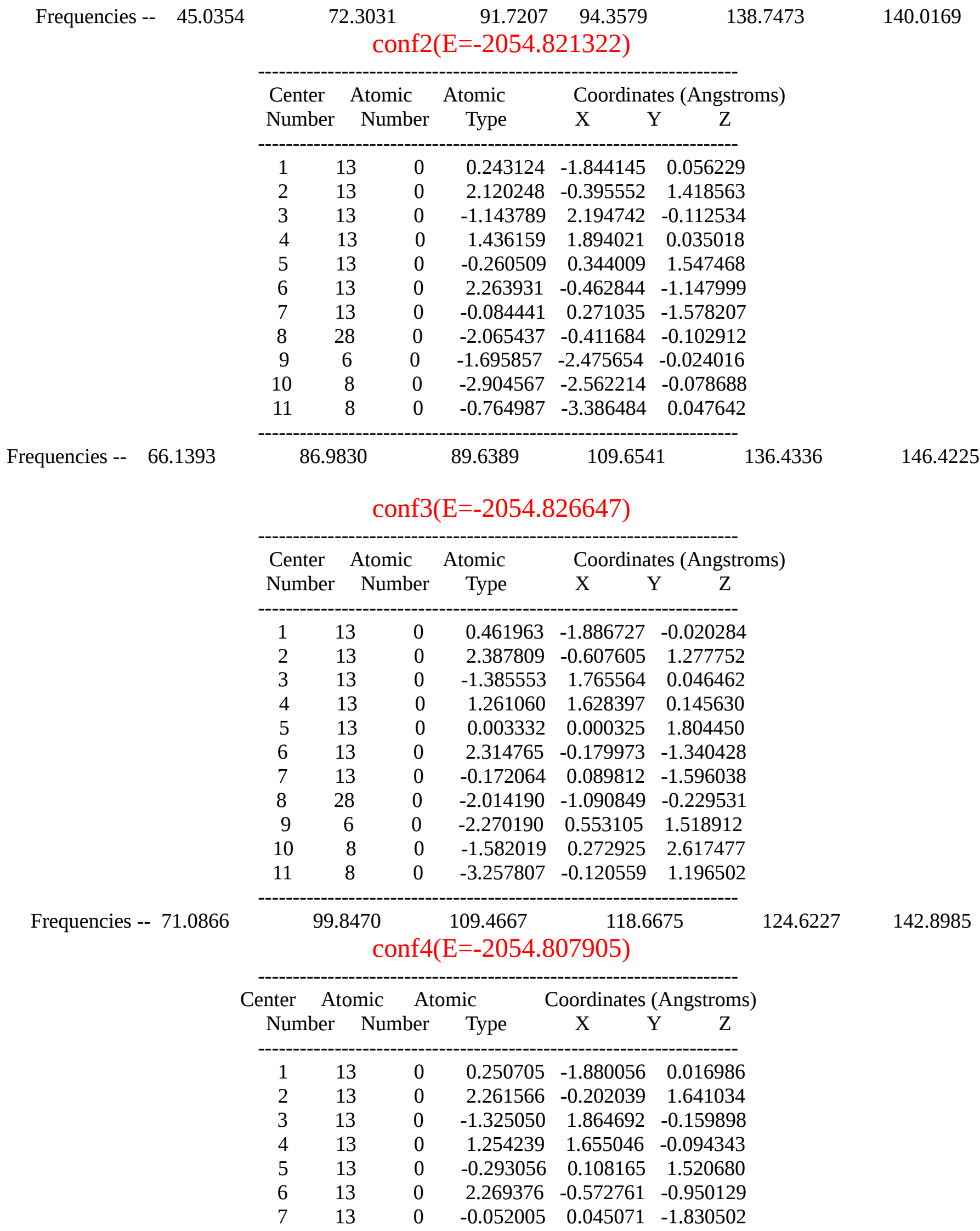




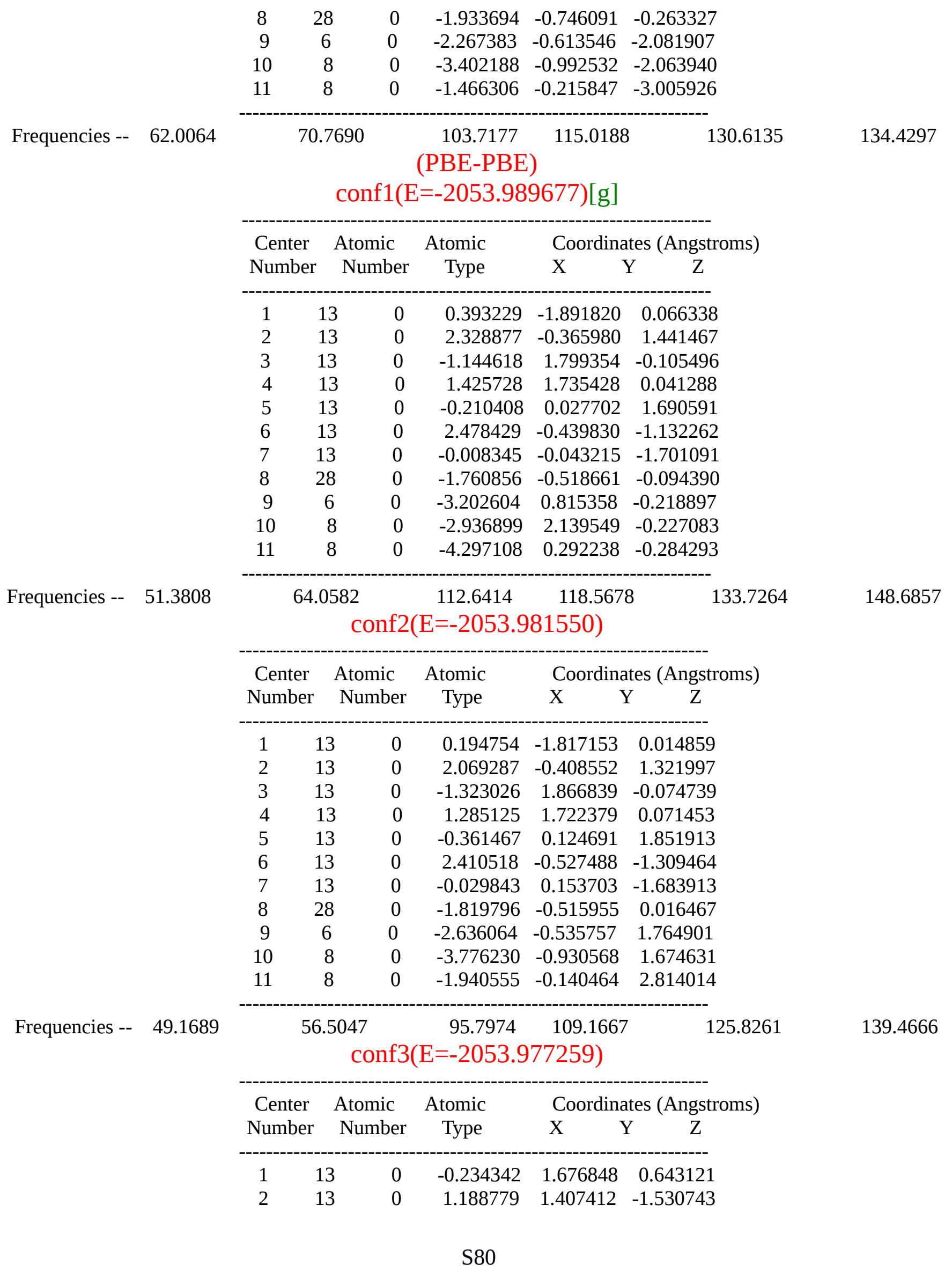




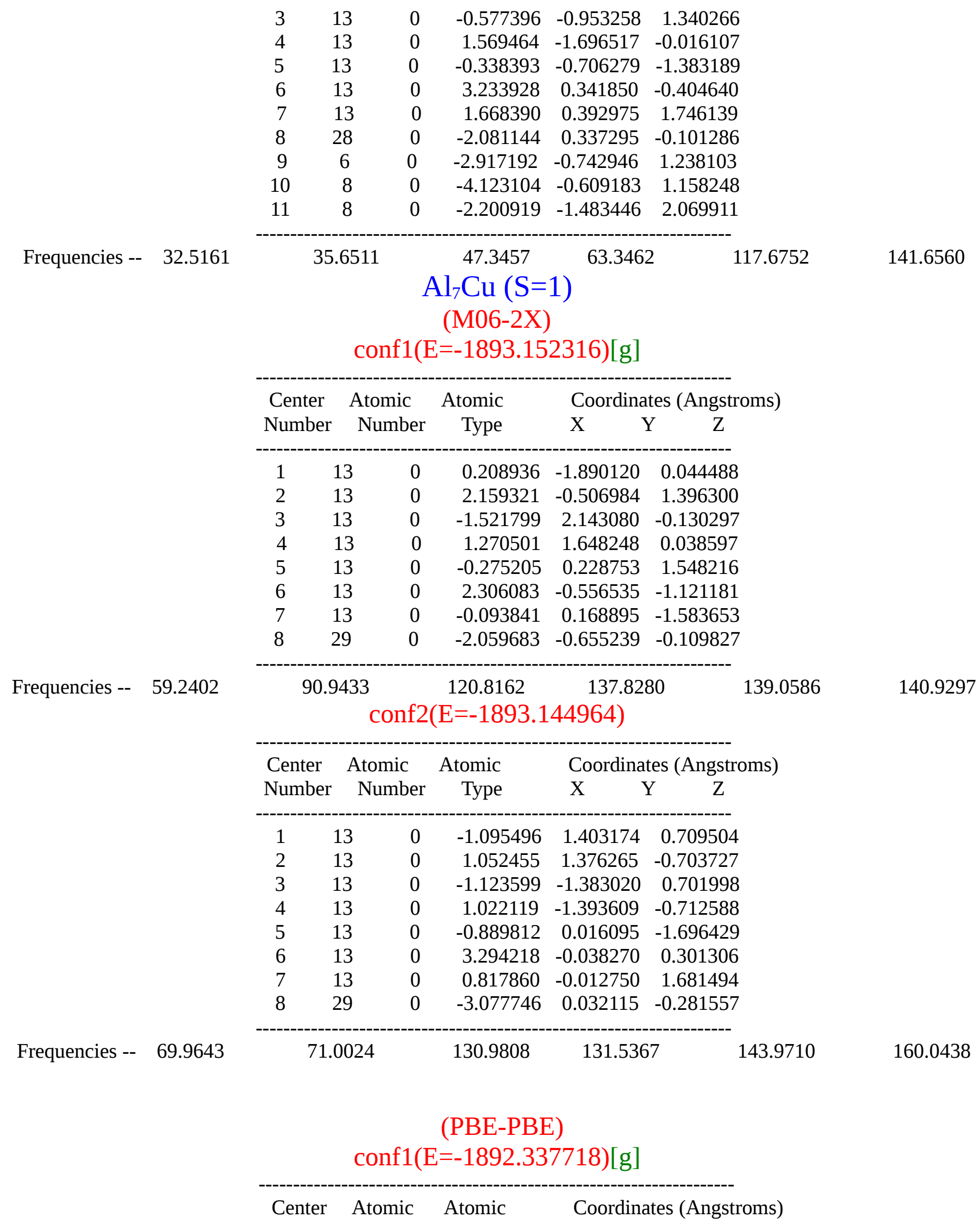




\begin{tabular}{rrrrrr} 
Number & Number & \multicolumn{1}{c}{ Type } & \multicolumn{1}{c}{ X } & Y & $Z$ \\
\hline 1 & 13 & 0 & 0.161290 & -1.893867 & 0.041581 \\
2 & 13 & 0 & 2.169682 & -0.521995 & 1.403267 \\
3 & 13 & 0 & -1.572050 & 2.078566 & -0.130199 \\
4 & 13 & 0 & 1.230015 & 1.649574 & 0.037789 \\
5 & 13 & 0 & -0.278868 & 0.226472 & 1.617542 \\
6 & 13 & 0 & 2.315663 & -0.572969 & -1.127957 \\
7 & 13 & 0 & -0.089255 & 0.167758 & -1.651917 \\
8 & 29 & 0 & -1.942163 & -0.553442 & -0.107464
\end{tabular}

Frequencies -- 80.9980

89.0453

$109.2295 \quad 125.3365$

133.7672

137.5187

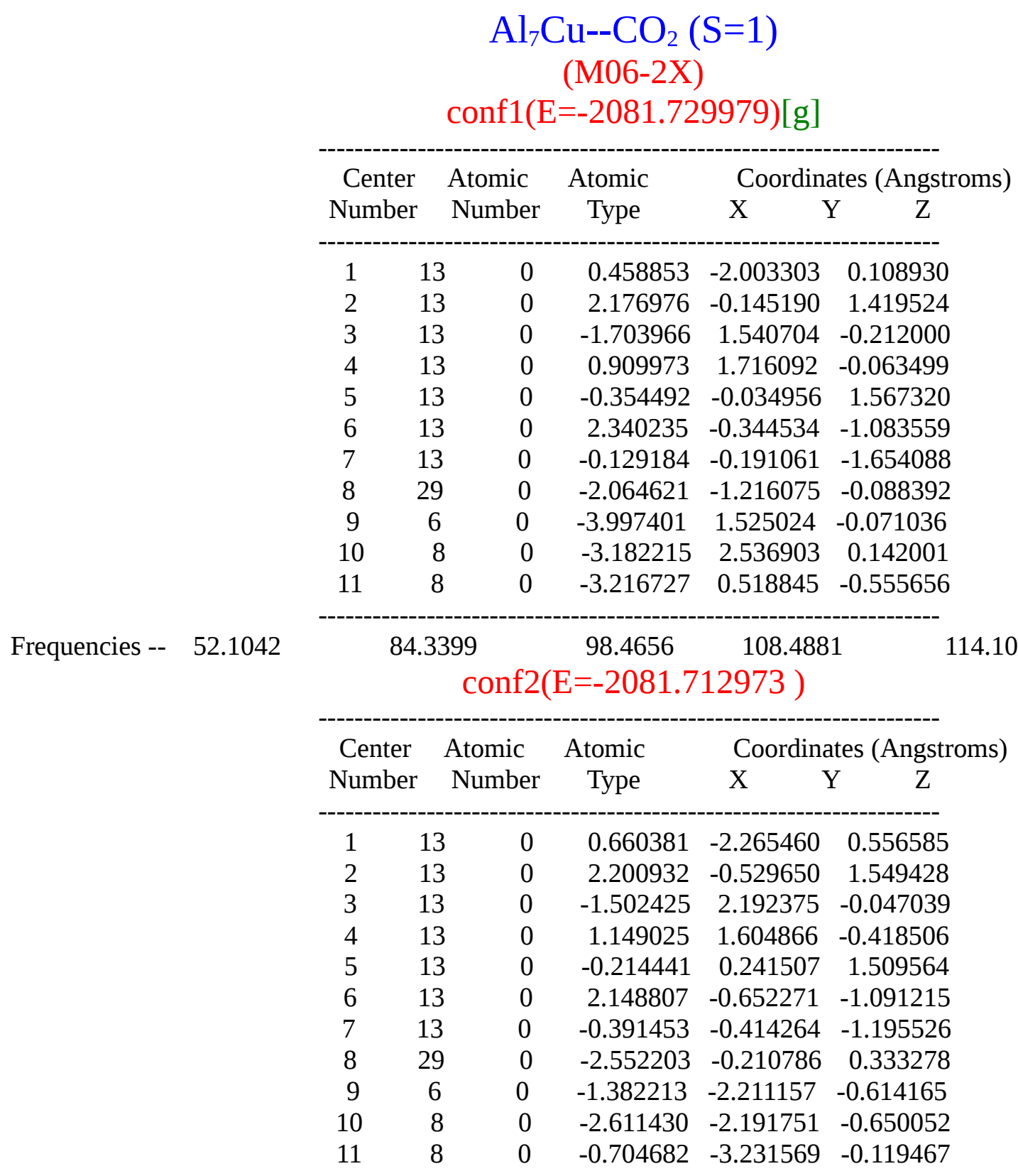




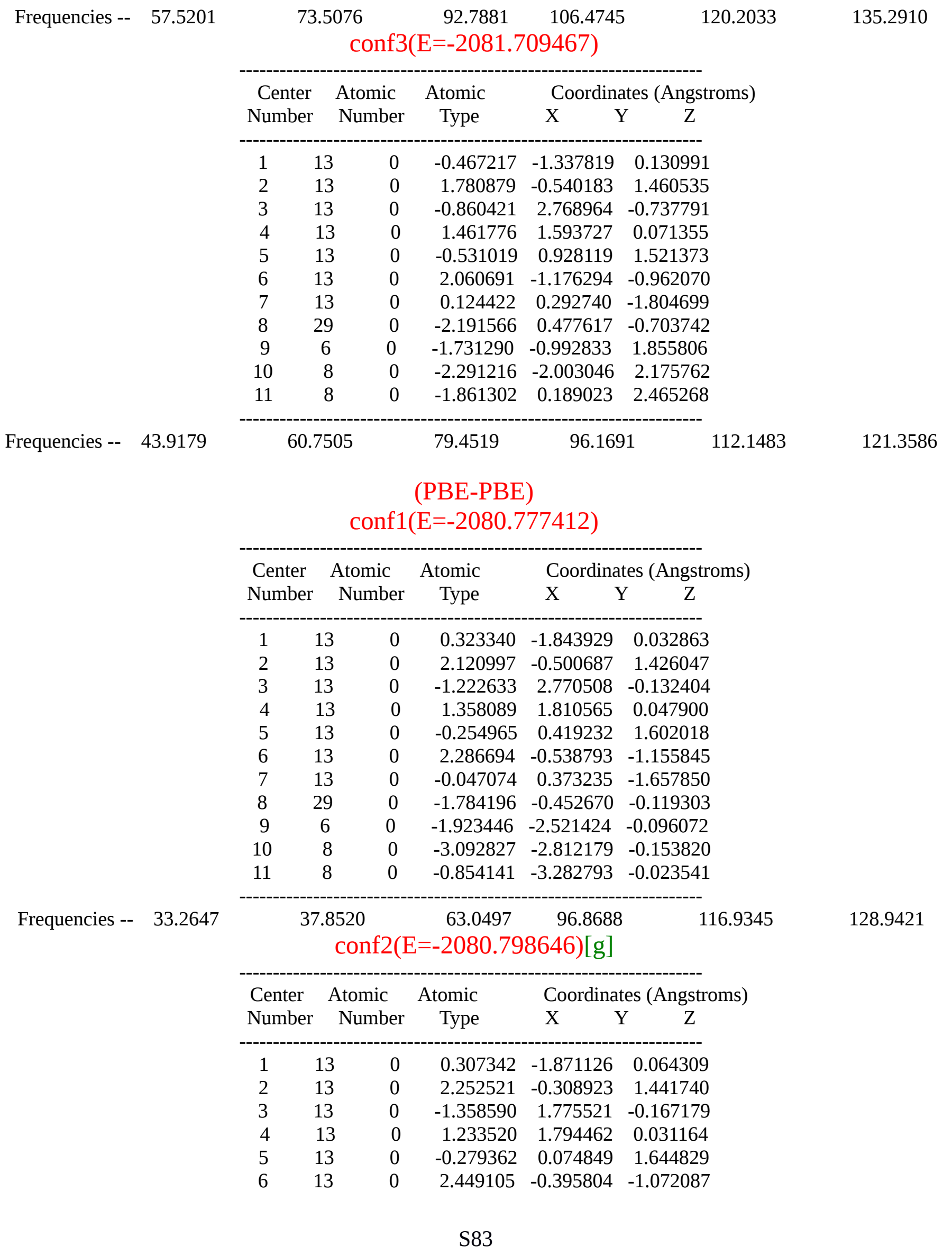




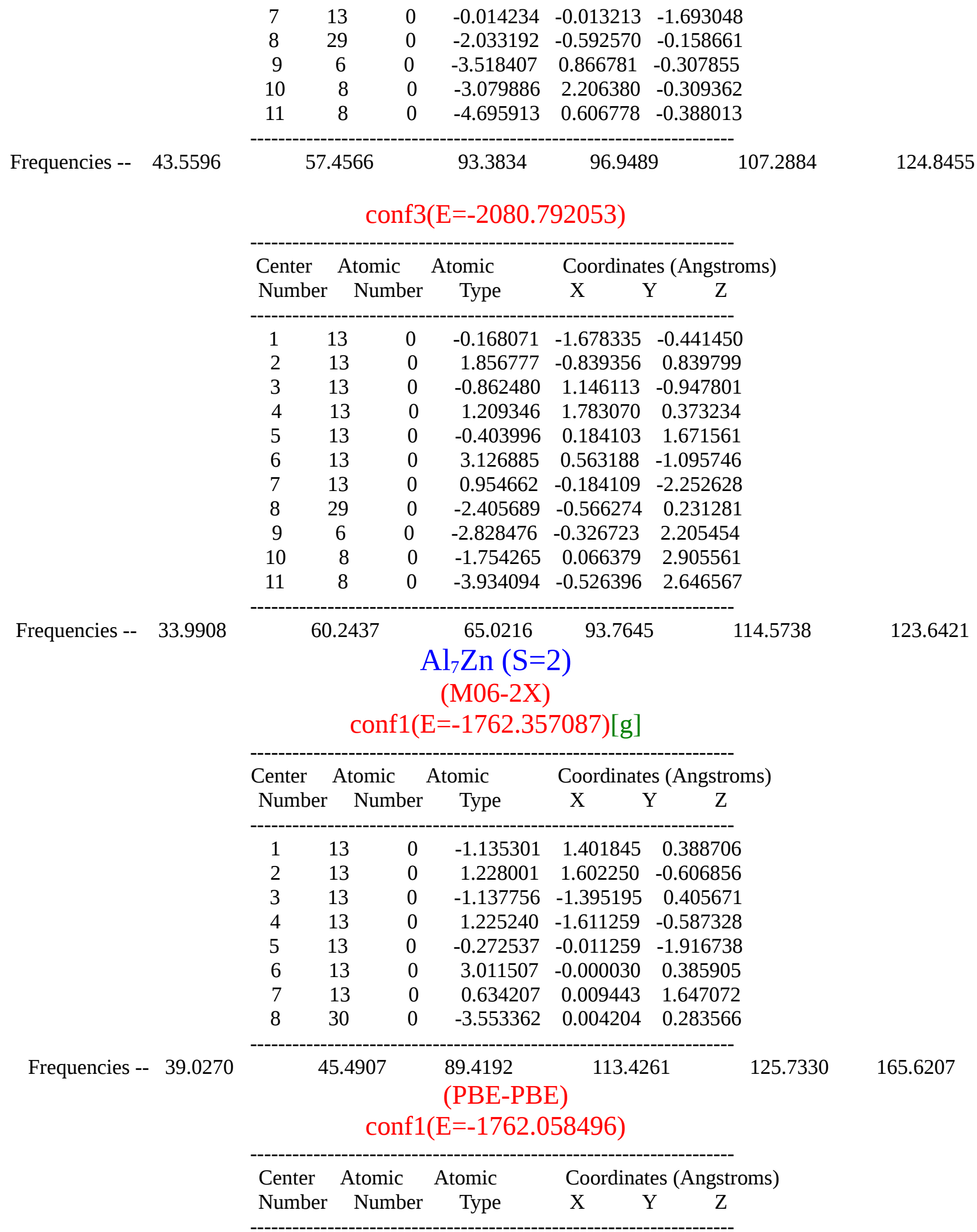




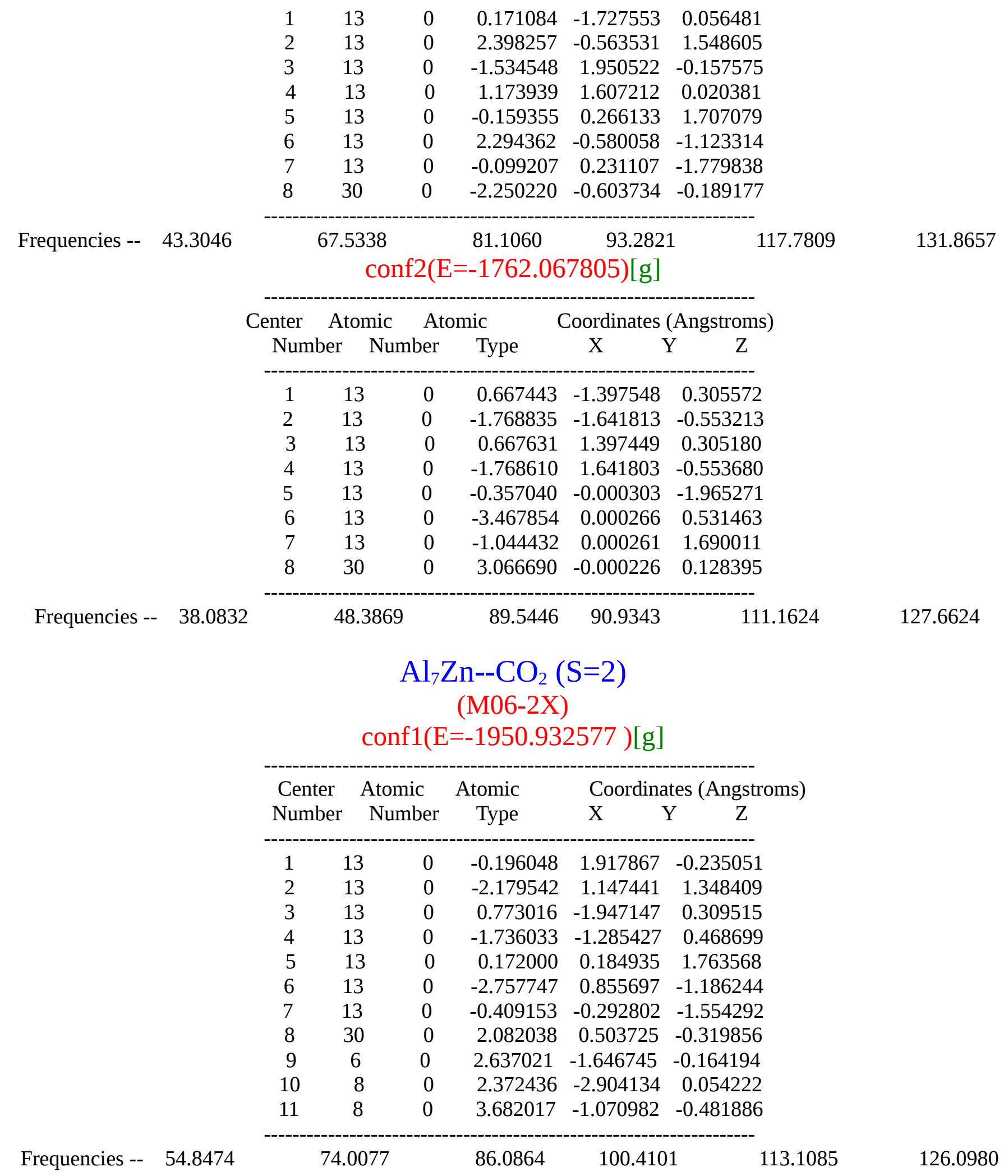




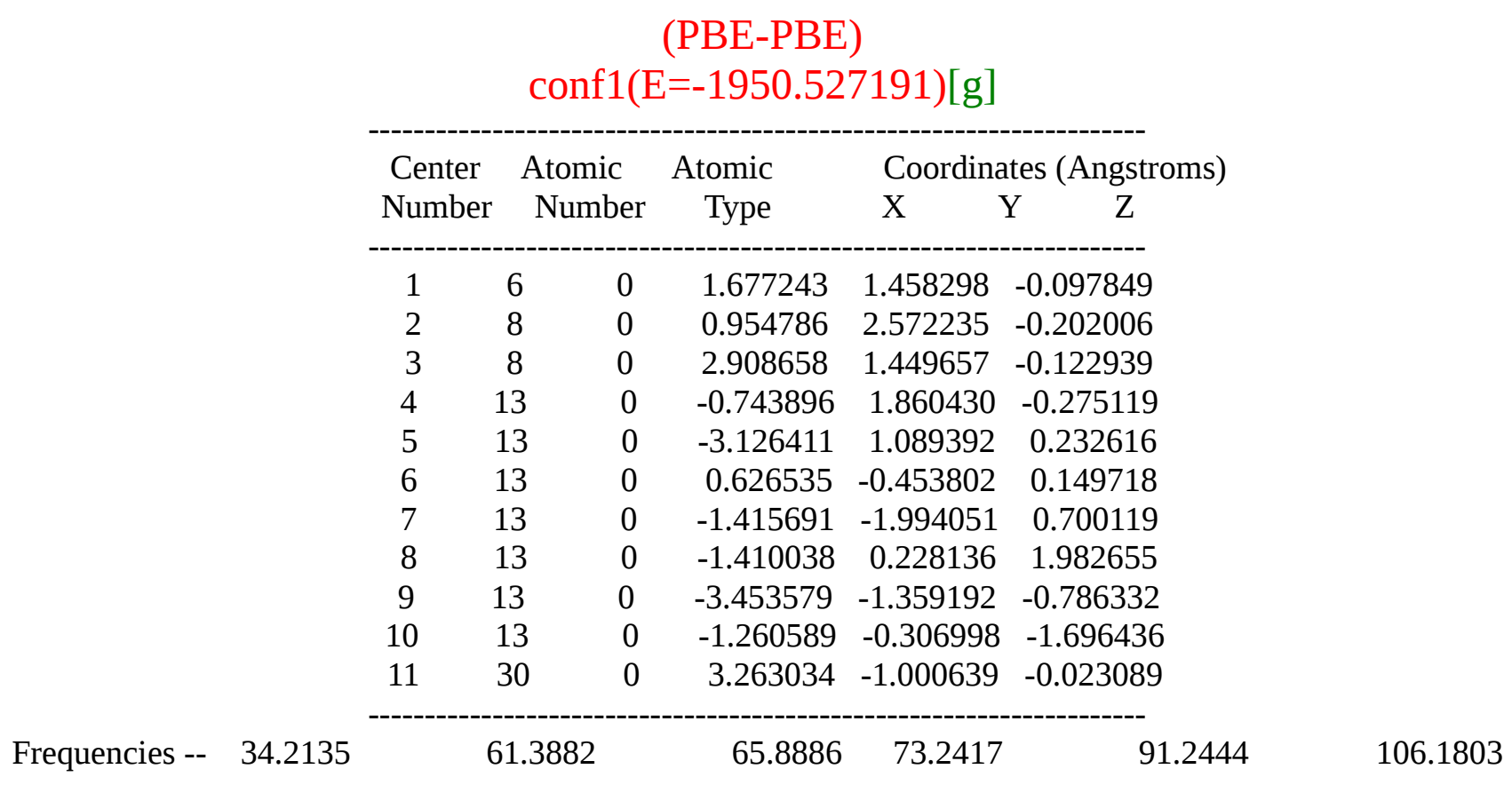


(ii) $\mathrm{Al}_{7} \mathrm{M}$ (b) cluster series

$\mathrm{Al}_{7} \mathrm{Sc}(\mathrm{S}=3)$

(M06-2X)

conf1(E=-1743.294497)[g]

\begin{tabular}{|c|c|c|c|}
\hline Center & Atomic & Atomic & Coordinates (Angstroms) \\
\hline Number & Number & Tyре & X \\
\hline
\end{tabular}

Frequencies -- 87.1394

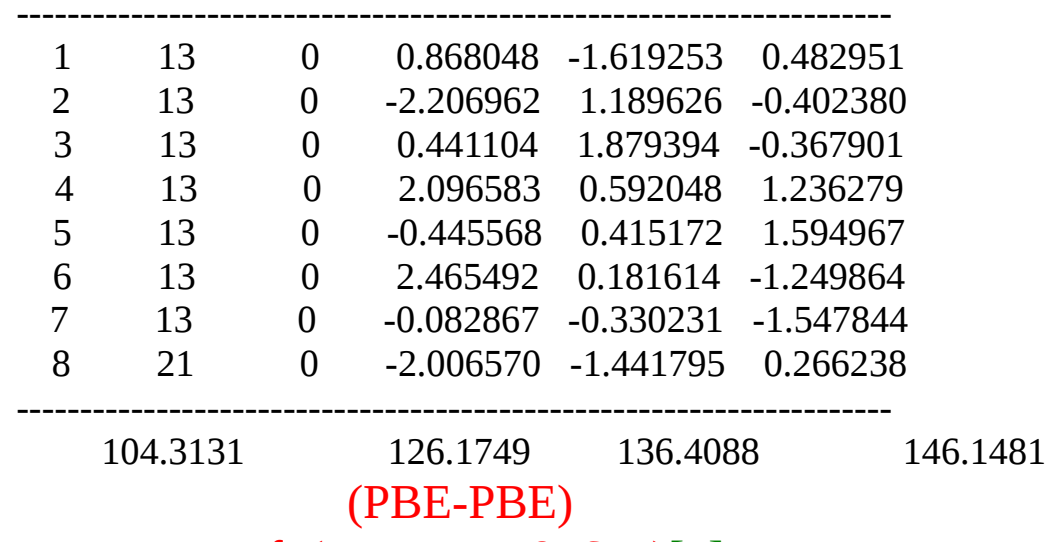

152.4444

conf1(E=-1742.591654)[g]

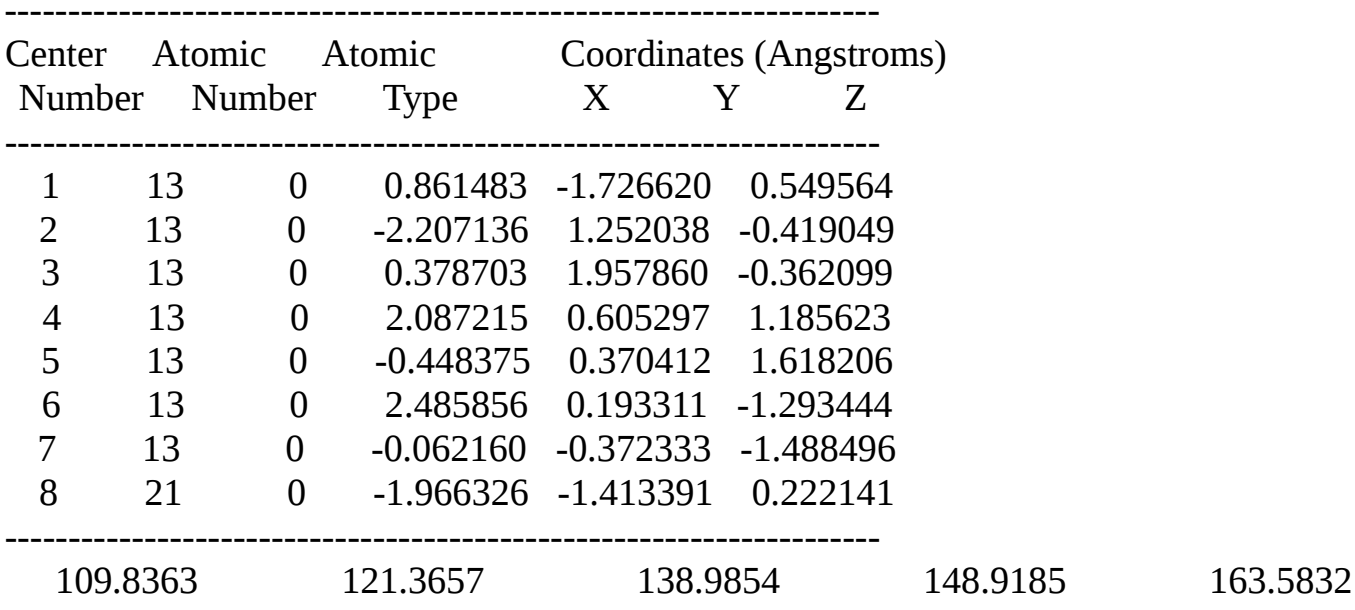

Frequencies -- 62.1174

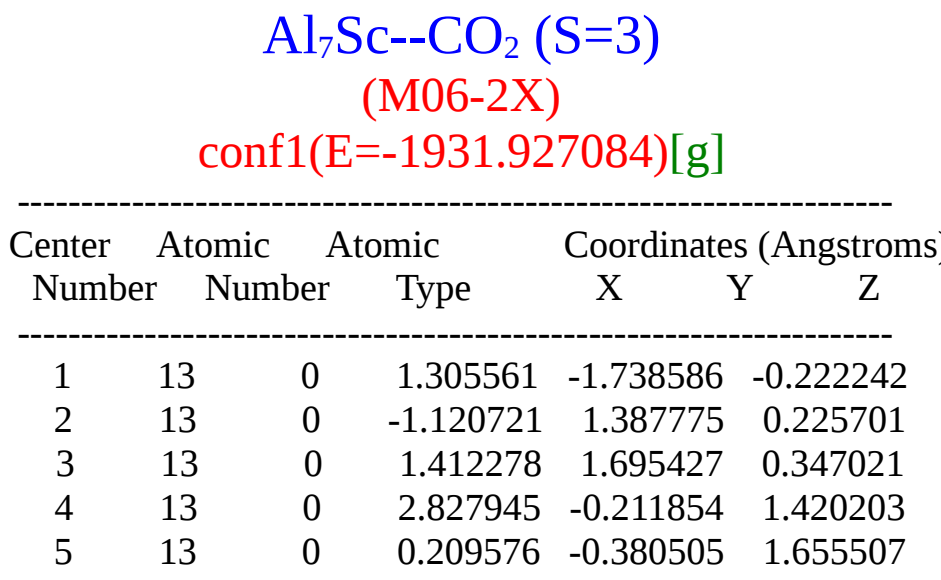




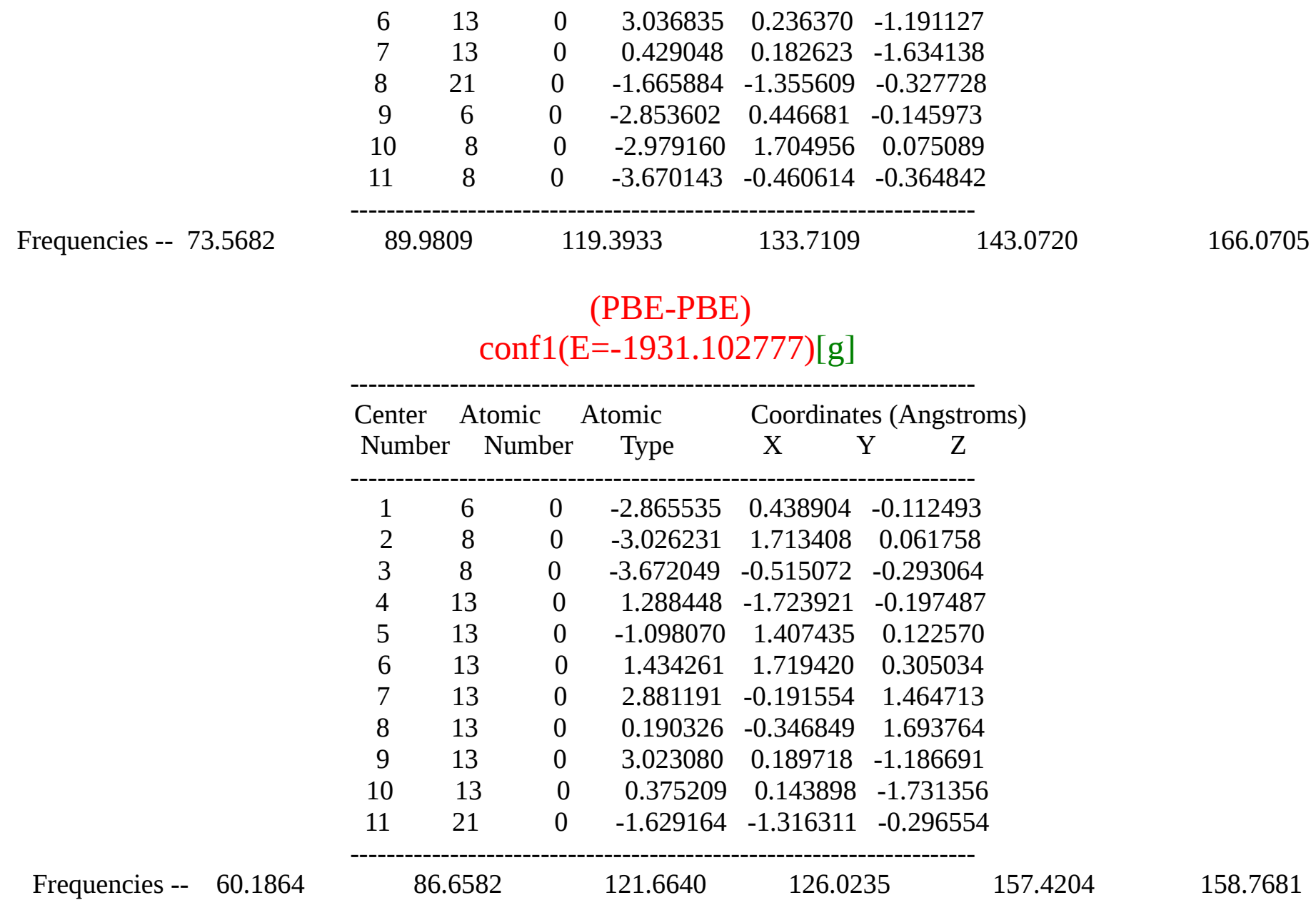

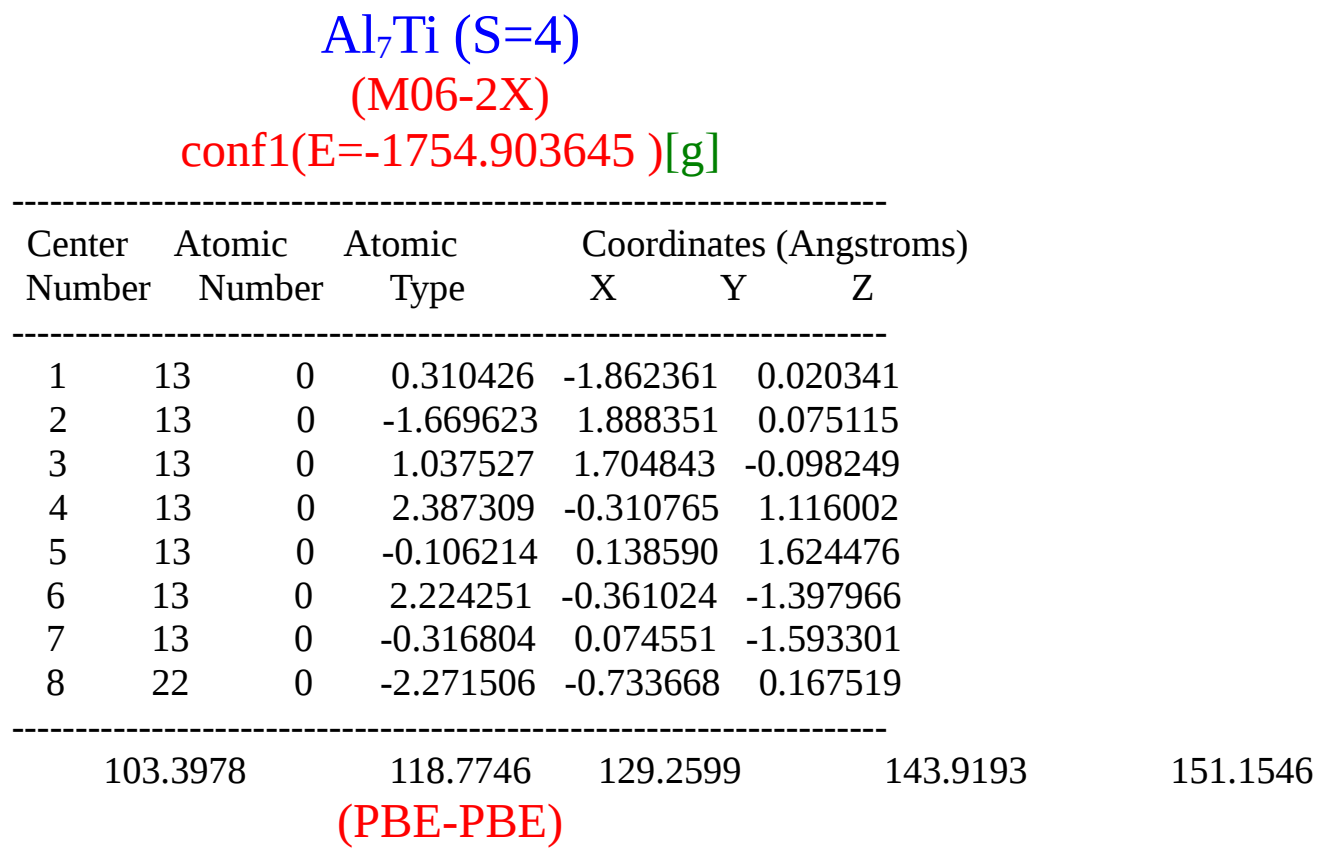




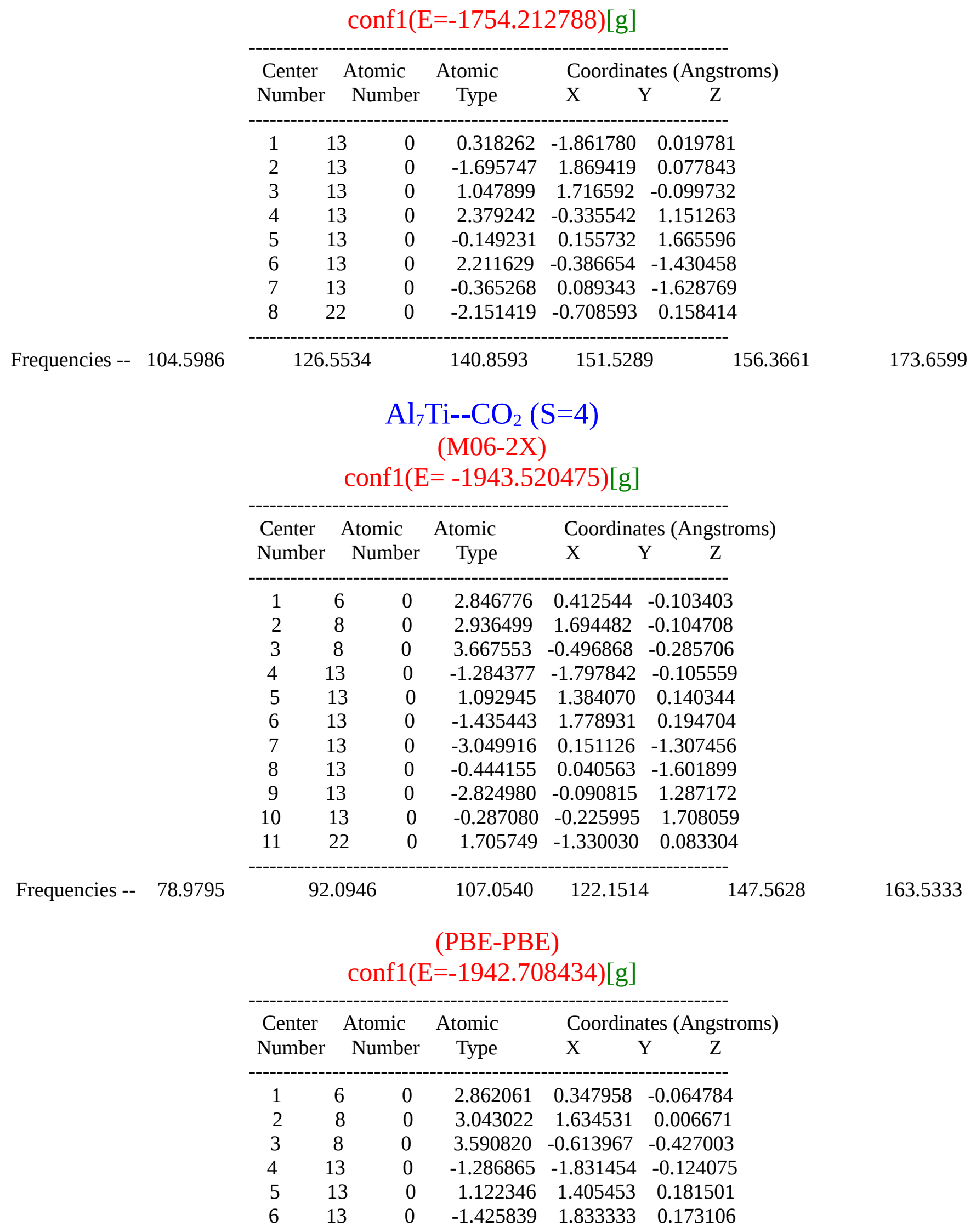




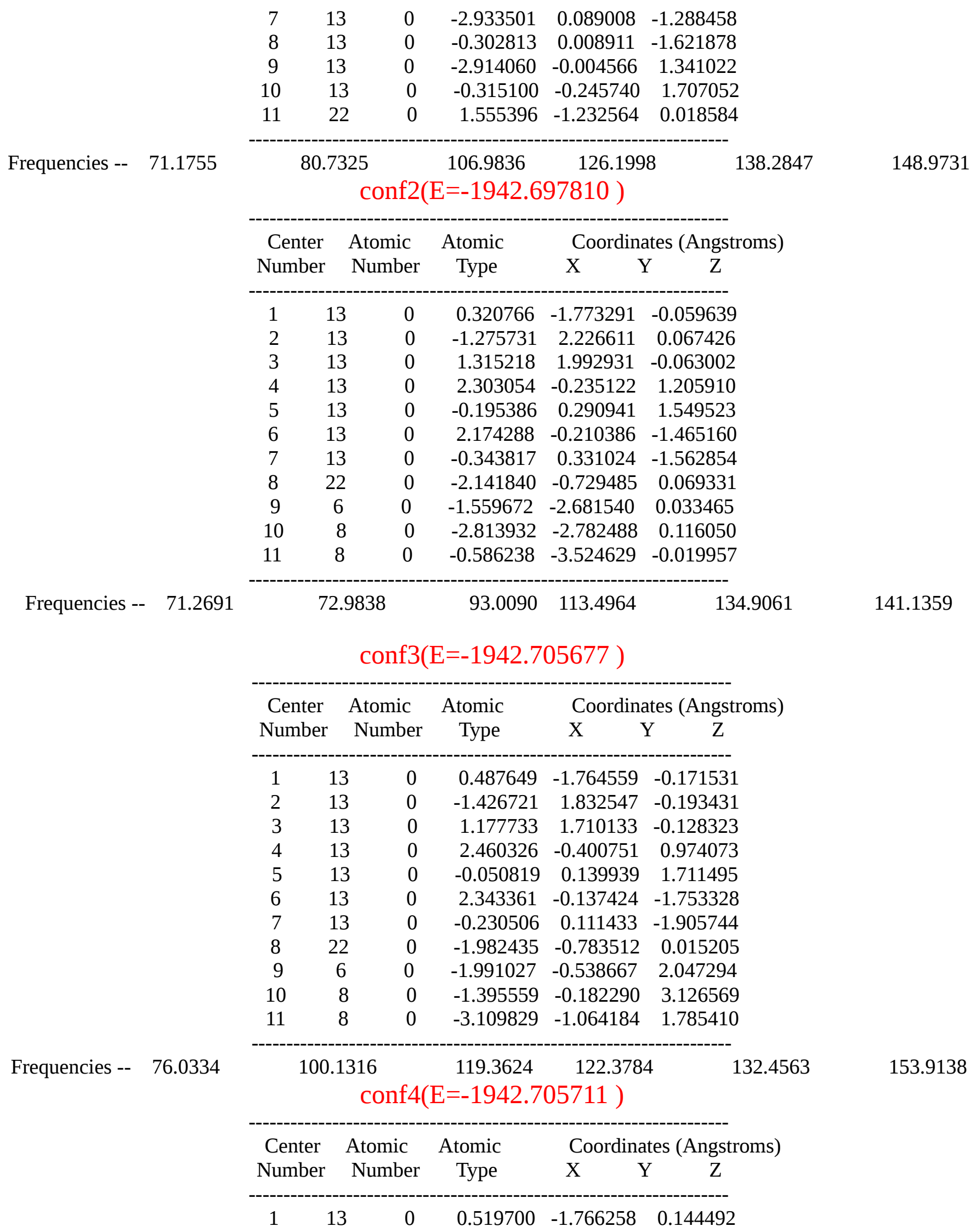




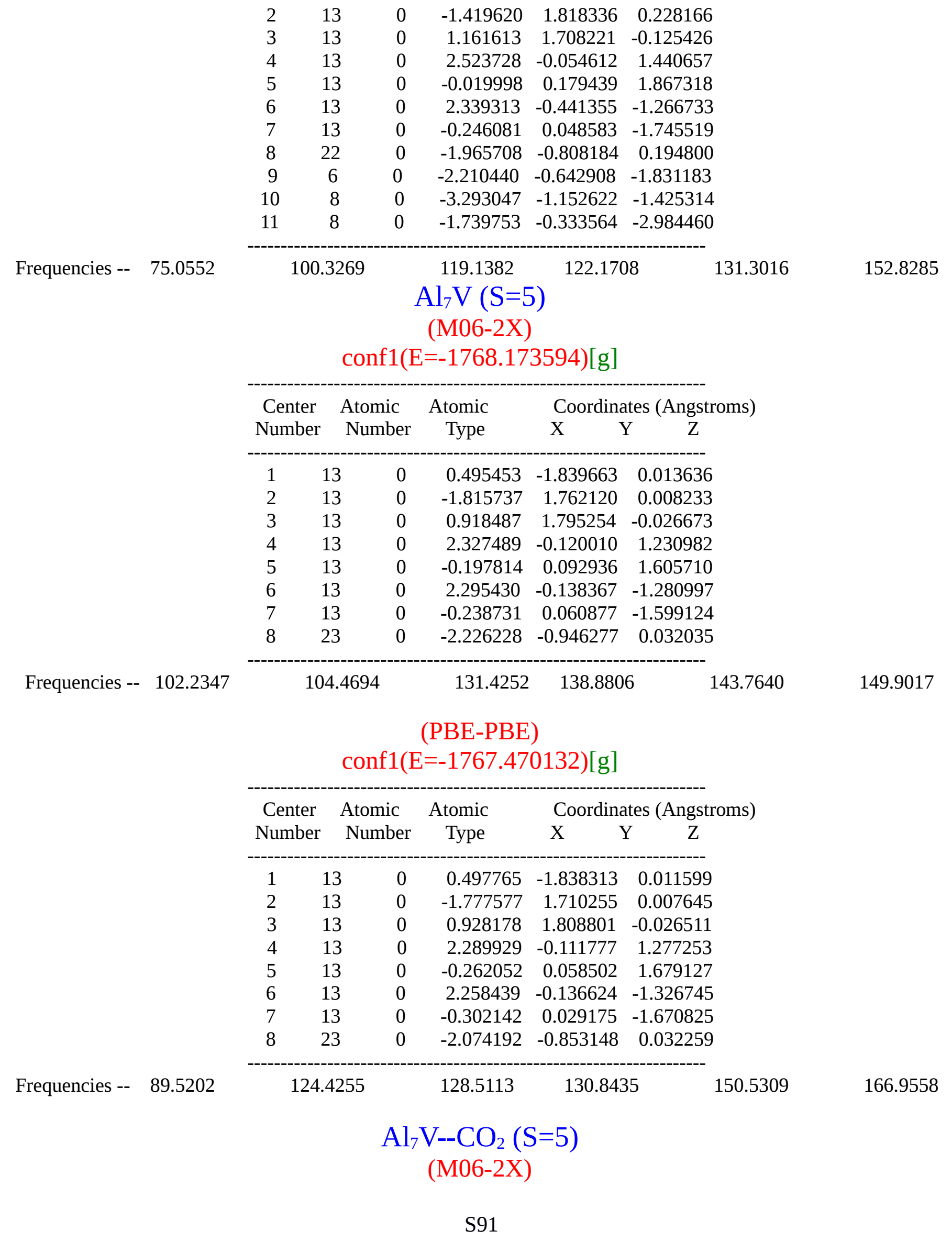




\section{conf1(E=-1956.783035)[g]}

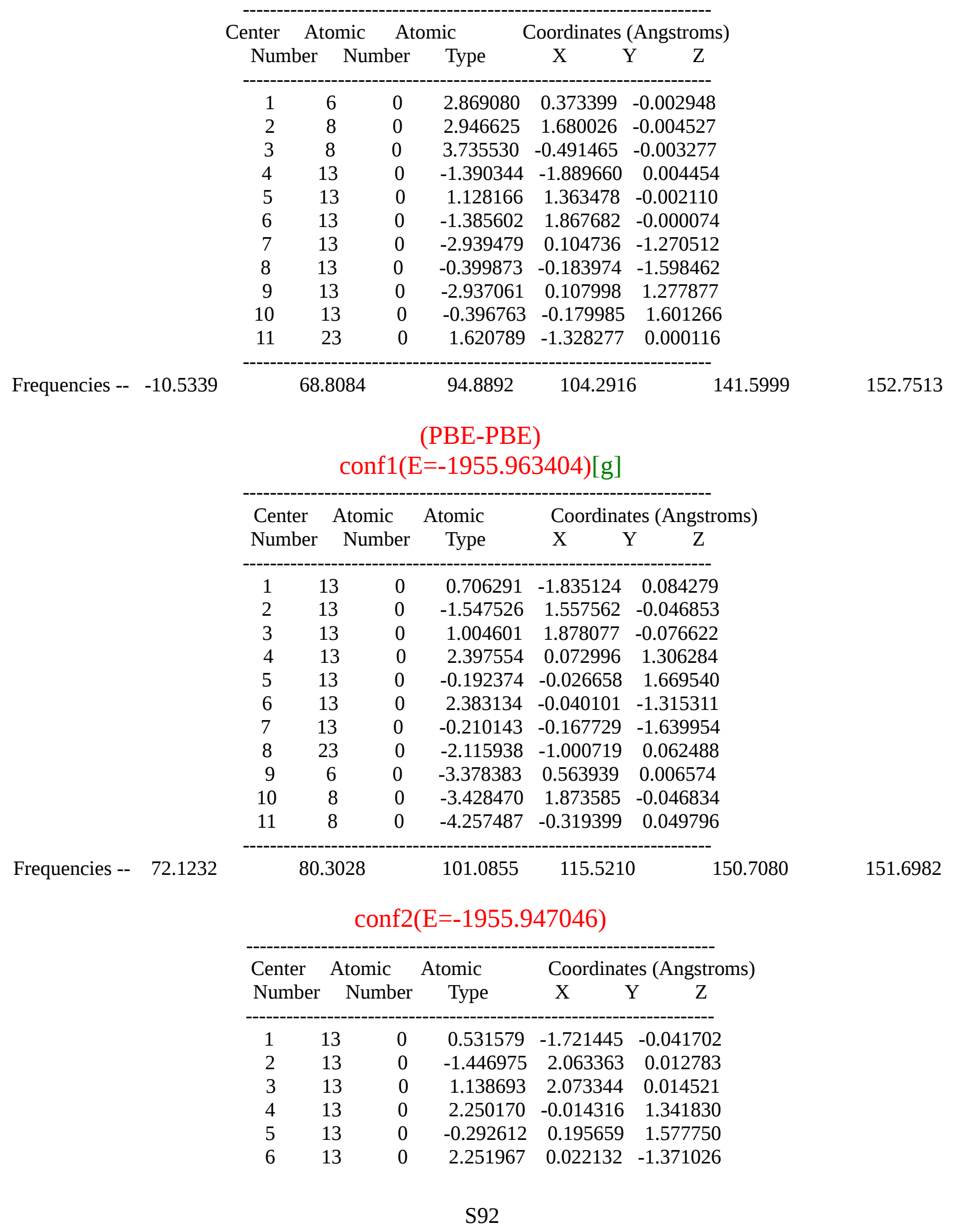




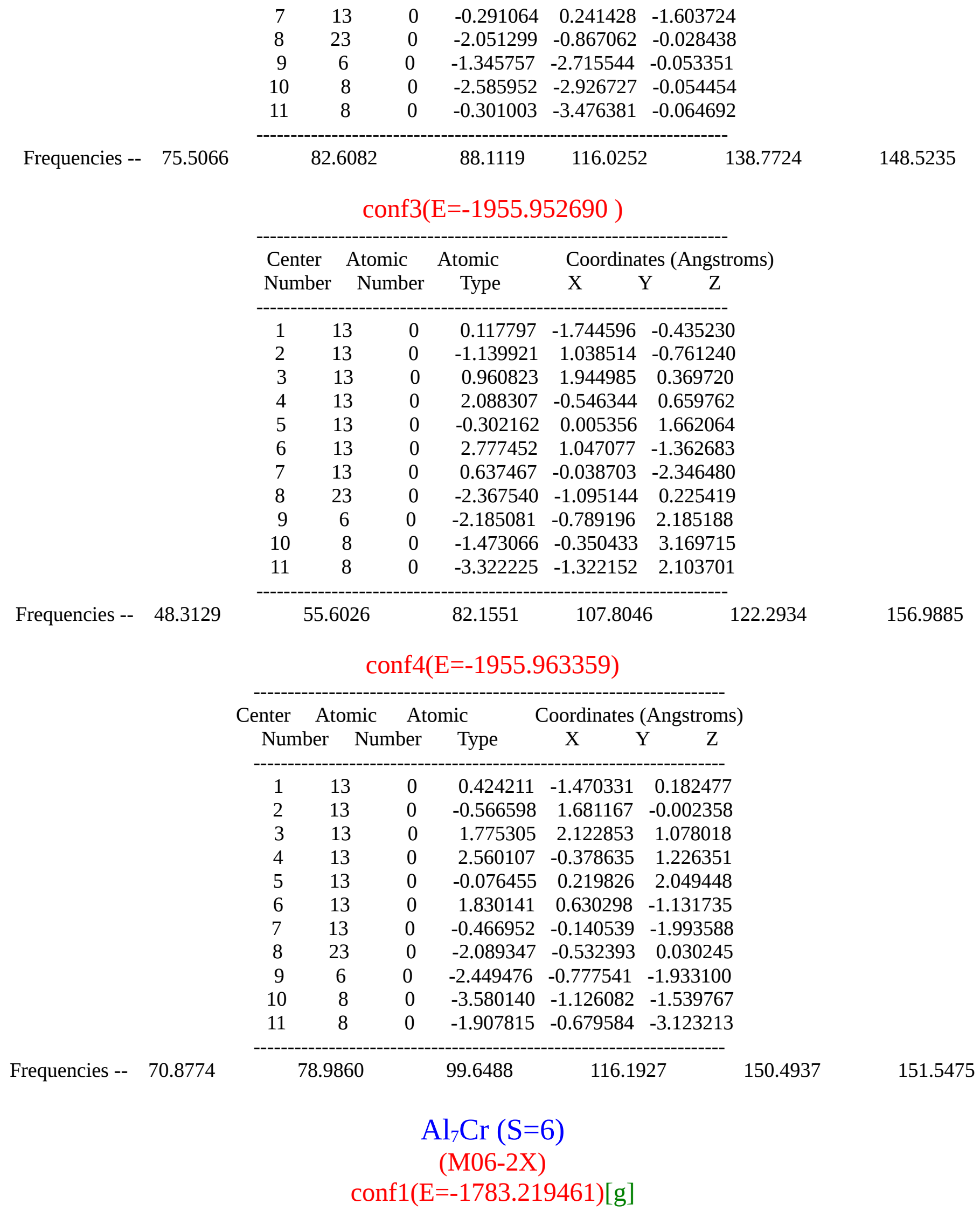




\begin{tabular}{|c|c|c|c|c|c|}
\hline \multirow{2}{*}{$\begin{array}{c}\text { Center } \\
\text { Number }\end{array}$} & \multirow{2}{*}{\multicolumn{2}{|c|}{$\begin{array}{l}\text { Atomic } \\
\text { Number }\end{array}$}} & \multirow{2}{*}{$\begin{array}{r}\text { Atomic } \\
\text { Type }\end{array}$} & \multicolumn{2}{|c|}{ Coordinates (Angstro } \\
\hline & & & & $\mathrm{X}$ & $\mathrm{Y} \quad \mathrm{Z}$ \\
\hline 1 & 13 & 0 & -0.167331 & 0.148000 & 1.567240 \\
\hline 2 & 13 & 0 & -1.627156 & 1.983472 & 0.012409 \\
\hline 3 & 13 & 0 & -0.175475 & 0.159778 & -1.564101 \\
\hline 4 & 13 & 0 & 2.318429 & -0.312039 & -1.261430 \\
\hline 5 & 13 & 0 & 1.140614 & 1.754701 & 0.004147 \\
\hline 6 & 13 & 0 & 2.324851 & -0.321578 & 1.248292 \\
\hline 7 & 13 & 0 & 0.383490 & -1.864017 & -0.007470 \\
\hline 8 & 24 & 0 & -2.295129 & -0.872893 & 0.003297 \\
\hline
\end{tabular}

Frequencies -- 81.3714

97.4119

117.8736

138.3044

141.0762

146.2197

(PBE-PBE)

$$
\operatorname{conf1}(\mathrm{E}=-1782.466270)[\mathrm{g}]
$$

\begin{tabular}{|c|c|c|c|c|c|}
\hline \multirow{2}{*}{$\begin{array}{l}\text { Center } \\
\text { Number }\end{array}$} & \multicolumn{2}{|c|}{ Atomic } & Atomic & \multicolumn{2}{|c|}{ Coordinates (Angstroms) } \\
\hline & & Number & Type & $\begin{array}{ll}X & Y\end{array}$ & $\mathrm{Y} \quad \mathrm{Z}$ \\
\hline 1 & 13 & 0 & -0.179905 & 0.135982 & 1.611171 \\
\hline 2 & 13 & 0 & -1.657210 & 1.916405 & 0.012187 \\
\hline 3 & 13 & 0 & -0.188165 & 0.148375 & -1.607839 \\
\hline 4 & 13 & 0 & 2.325693 & -0.321803 & -1.272471 \\
\hline 5 & 13 & 0 & 1.108193 & 1.769084 & 0.004343 \\
\hline 6 & 13 & 0 & 2.332148 & -0.330865 & 1.259086 \\
\hline 7 & 13 & 0 & 0.338844 & -1.866897 & -0.007438 \\
\hline 8 & 24 & 0 & -2.177305 & -0.774855 & 0.003344 \\
\hline
\end{tabular}

149.9981

Frequencies -- 88.1213

\begin{tabular}{|c|c|c|c|c|c|}
\hline \multirow{3}{*}{$\begin{array}{l}\text { Center } \\
\text { Numbe }\end{array}$} & \multicolumn{5}{|c|}{$\begin{aligned} & \mathrm{Al}_{7} \mathrm{Cr}--\mathrm{CO}_{2}(\mathrm{~S}=6) \\
&(\mathrm{M} 06-2 \mathrm{X}) \\
& \mathrm{hf} 1(\mathrm{E}=-1971.805771)[\mathrm{g}]\end{aligned}$} \\
\hline & Atomi & & mic & Coordinates & (Angstroms) \\
\hline & & Jumber & Type & $\mathrm{X}$ & $\begin{array}{l}Y \\
Y\end{array}$ \\
\hline 1 & 6 & 0 & -2.883241 & 0.539411 & 0.097729 \\
\hline 2 & 8 & 0 & -2.834093 & 1.879407 & 0.095563 \\
\hline 3 & 8 & 0 & -3.862535 & -0.176348 & 0.119163 \\
\hline 4 & 13 & 0 & 0.514180 & -0.165356 & 1.605918 \\
\hline 5 & 13 & 0 & -1.093097 & 1.375560 & 0.051147 \\
\hline 6 & 13 & 0 & 0.416734 & -0.160010 & -1.602664 \\
\hline 7 & 13 & 0 & 2.944238 & 0.009627 & -1.326608 \\
\hline 8 & 13 & 0 & 1.452841 & 1.835310 & -0.024317 \\
\hline 9 & 13 & 0 & 3.021813 & 0.002810 & 1.177039 \\
\hline 10 & 13 & 0 & 1.348816 & -1.953146 & -0.028578 \\
\hline
\end{tabular}




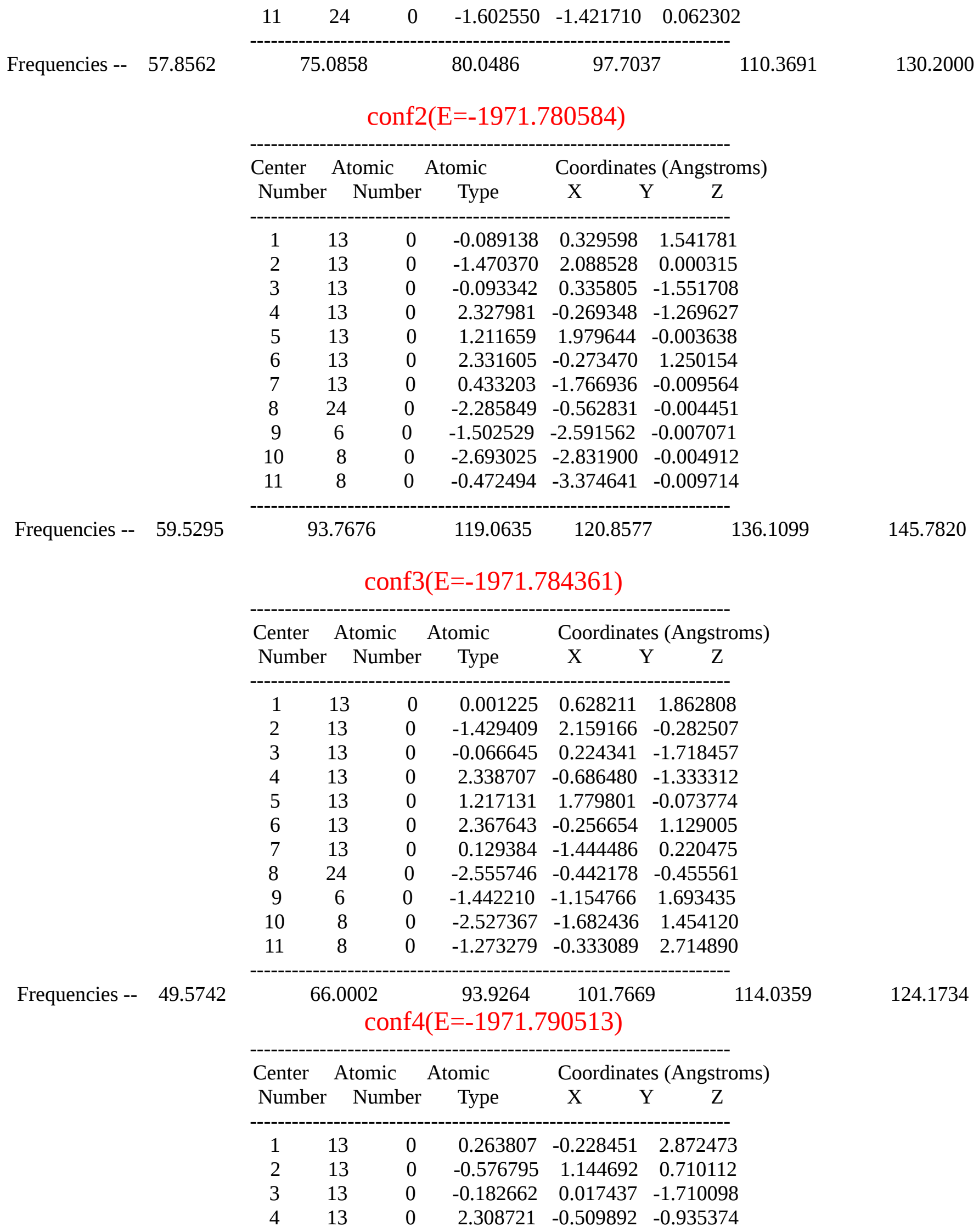




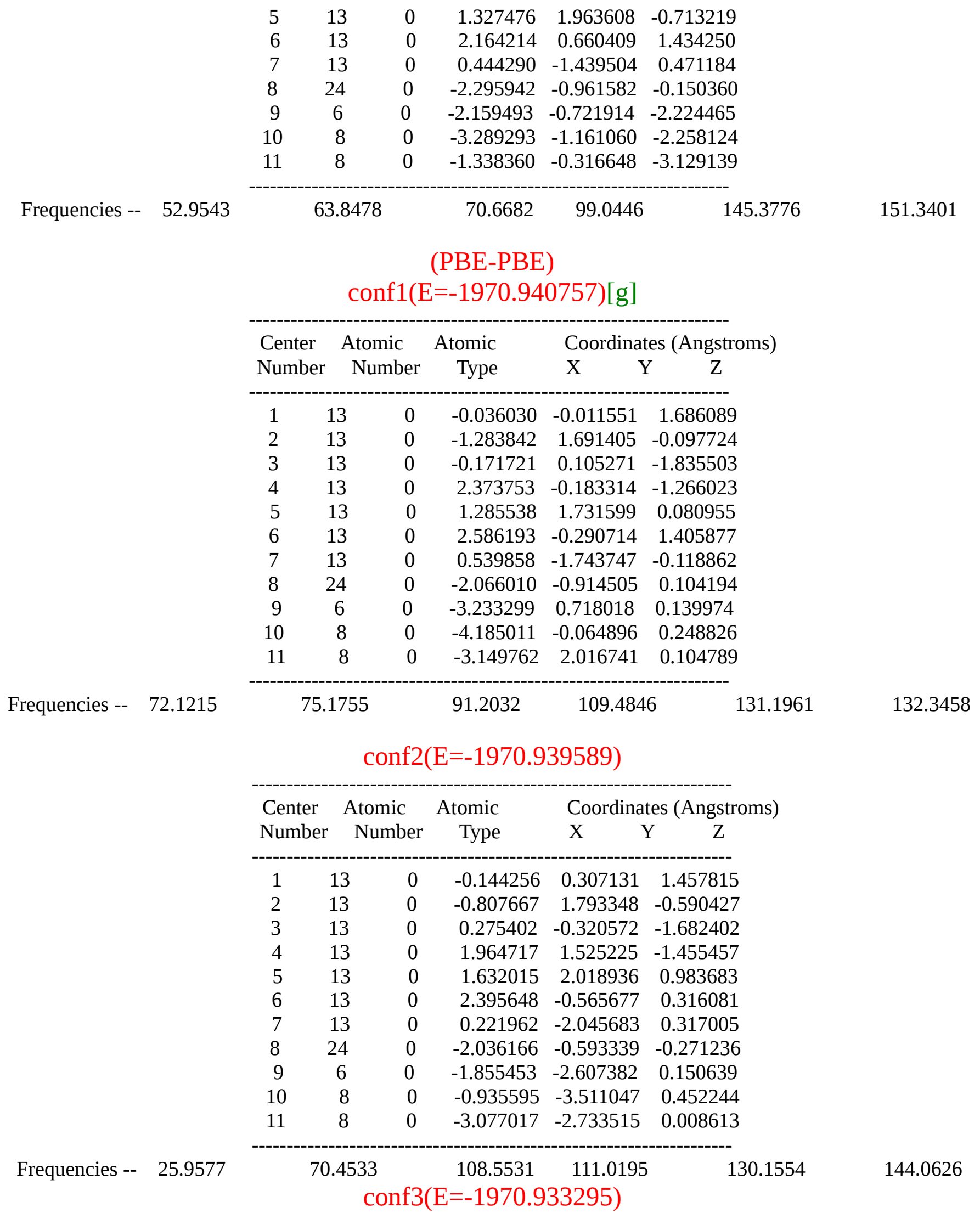




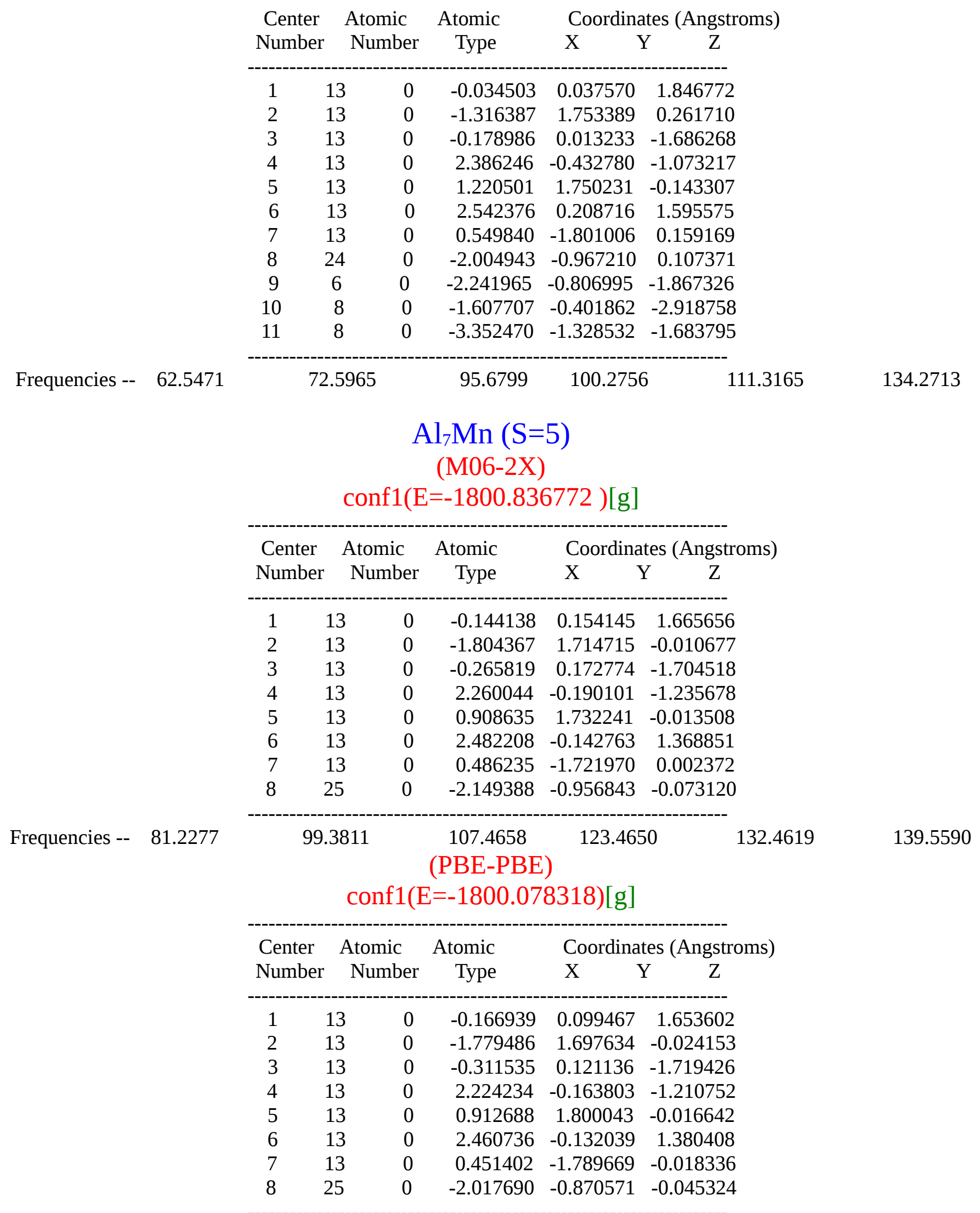




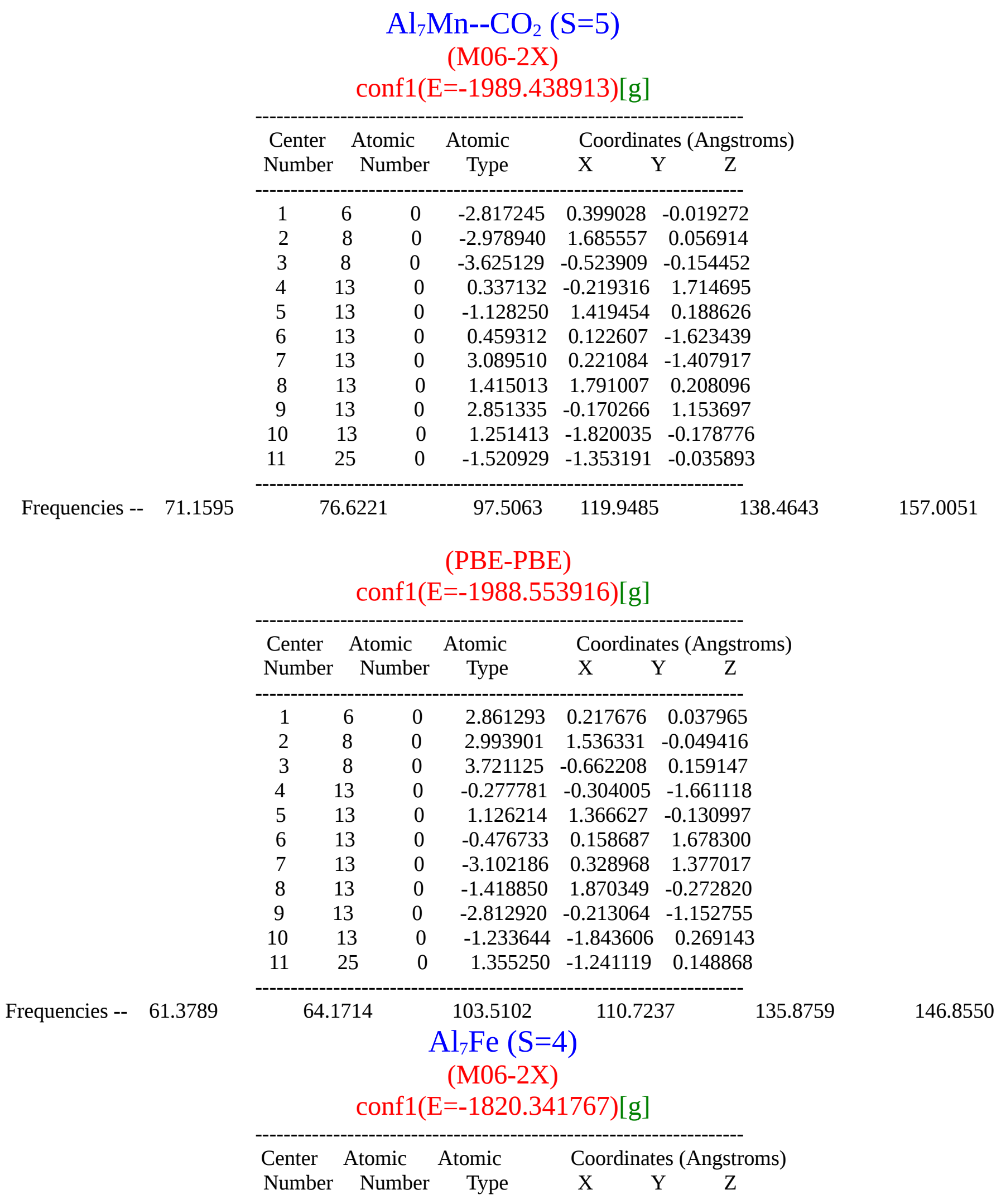




\begin{tabular}{|c|c|c|c|c|c|c|}
\hline & \\
\hline & 1 & 13 & -0.167200 & 0.153540 & 1.589855 & \\
\hline & 2 & 13 & -1.716187 & 1.900917 & 0.017127 & \\
\hline & 3 & 13 & -0.163465 & 0.186001 & -1.590213 & \\
\hline & 4 & 13 & 2.337851 & -0.249619 & -1.254159 & \\
\hline & 5 & 13 & 1.065862 & 1.784444 & 0.017283 & \\
\hline & 6 & 13 & 2.335807 & -0.275109 & 1.254778 & \\
\hline & 7 & 13 & 0.393839 & -1.836771 & -0.019097 & \\
\hline & 8 & 26 & -2.063099 & -0.848767 & -0.016558 & \\
\hline \multirow[t]{12}{*}{ Frequencies -- 100.4863} & \multicolumn{2}{|c|}{101.7199} & 125.6439 & 146.1631 & 148.1224 & 150.9862 \\
\hline & \multicolumn{5}{|c|}{$\begin{array}{c}(\mathrm{PBE}-\mathrm{PBE}) \\
\operatorname{conf1}(\mathrm{E}=-1819.608831)[\mathrm{g}]\end{array}$} & \\
\hline & \multirow{2}{*}{$\begin{array}{l}\text { Center } \\
\text { Number }\end{array}$} & \multirow{2}{*}{$\begin{array}{l}\text { Atomic } \\
\text { Number }\end{array}$} & Atomic & \multicolumn{2}{|c|}{ Coordinates (Angstroms) } & \\
\hline & & & Type & $X \quad Y$ & $\mathrm{Y} \quad \mathrm{Z}$ & \\
\hline & 1 & 13 & -0.242458 & 0.122825 & 1.693295 & \\
\hline & 2 & 13 & -1.680465 & 1.819012 & 0.001656 & \\
\hline & 3 & 13 & -0.176625 & 0.107341 & -1.622556 & \\
\hline & 4 & 13 & 2.379575 & -0.196342 & -1.349099 & \\
\hline & 5 & 13 & 1.021305 & 1.807612 & 0.047891 & \\
\hline & 6 & 13 & 2.255744 & -0.285972 & 1.218522 & \\
\hline & 7 & 13 & 0.326425 & -1.878246 & 0.016785 & \\
\hline & 8 & 26 & -1.860094 & -0.681594 & -0.007478 & \\
\hline Frequencies -- 76.6382 & 118. & 3.6169 & 131.6874 & 138.659 & 150.2900 & 158.4694 \\
\hline
\end{tabular}

\begin{tabular}{|c|c|c|c|c|c|}
\hline \multirow{2}{*}{$\begin{array}{l}\text { Center } \\
\text { Number }\end{array}$} & \multirow{2}{*}{\multicolumn{2}{|c|}{$\begin{array}{l}\text { Atomic } \\
\text { Number }\end{array}$}} & \multirow{2}{*}{$\begin{array}{r}\text { Atomic } \\
\text { Type }\end{array}$} & \multicolumn{2}{|c|}{ Coordinates (Angstroms) } \\
\hline & & & & $X \quad Y$ & Y $\quad Z$ \\
\hline 1 & 6 & 0 & -2.822236 & 0.423466 & -0.062473 \\
\hline 2 & 8 & 0 & -2.965683 & 1.712487 & -0.067744 \\
\hline 3 & 8 & 0 & -3.627982 & -0.504211 & -0.116409 \\
\hline 4 & 13 & 0 & 0.283831 & -0.114637 & 1.694557 \\
\hline 5 & 13 & 0 & -1.115759 & 1.468596 & 0.074006 \\
\hline 6 & 13 & 0 & 0.436685 & 0.048383 & -1.665162 \\
\hline 7 & 13 & 0 & 3.065456 & 0.091115 & -1.401527 \\
\hline 8 & 13 & 0 & 1.430239 & 1.790831 & 0.083089 \\
\hline 9 & 13 & 0 & 2.807651 & -0.123680 & 1.186840 \\
\hline 10 & 13 & 0 & 1.191104 & -1.804771 & -0.099883 \\
\hline 11 & 26 & 0 & -1.510184 & -1.227184 & 0.018862 \\
\hline
\end{tabular}




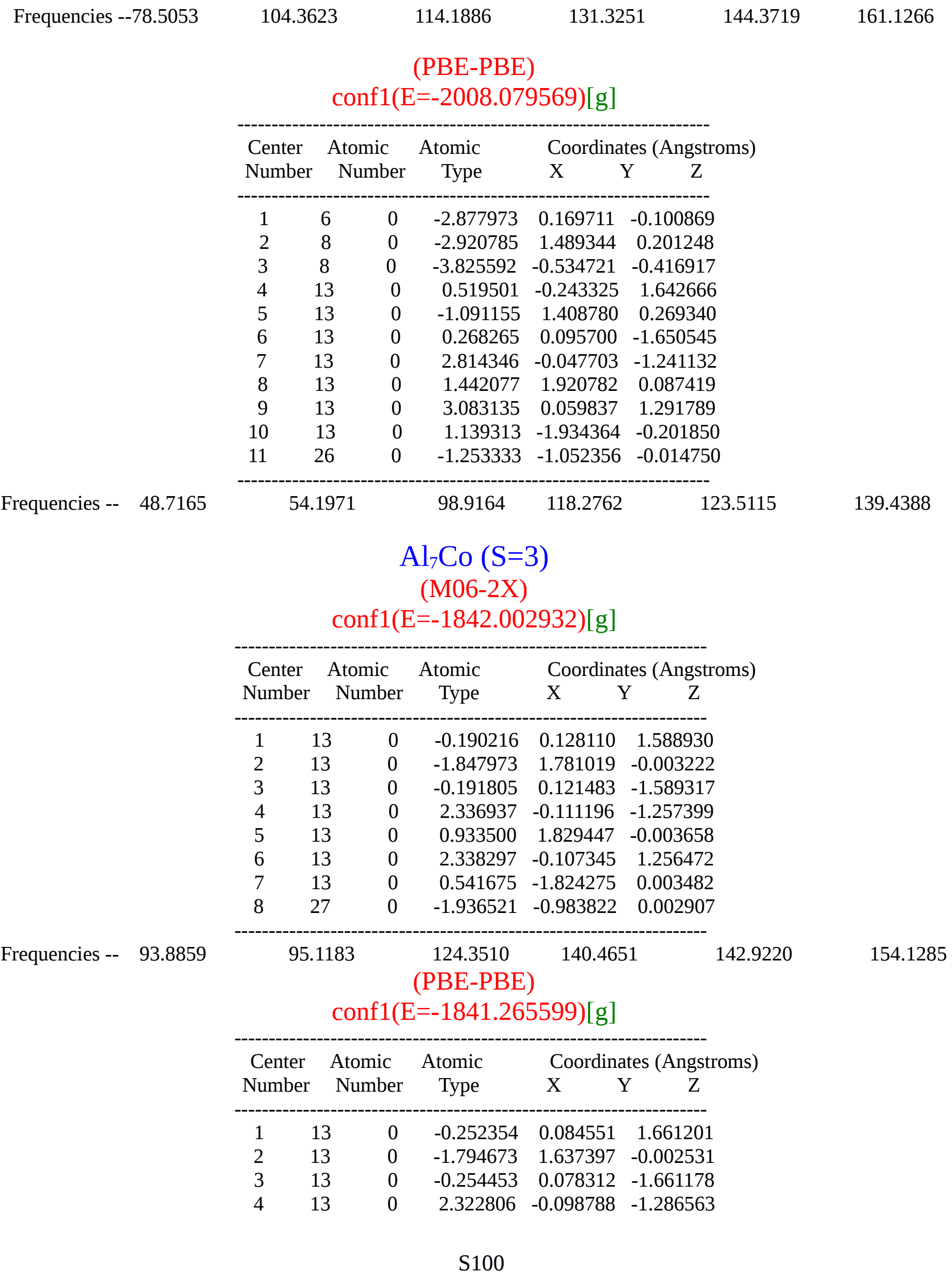




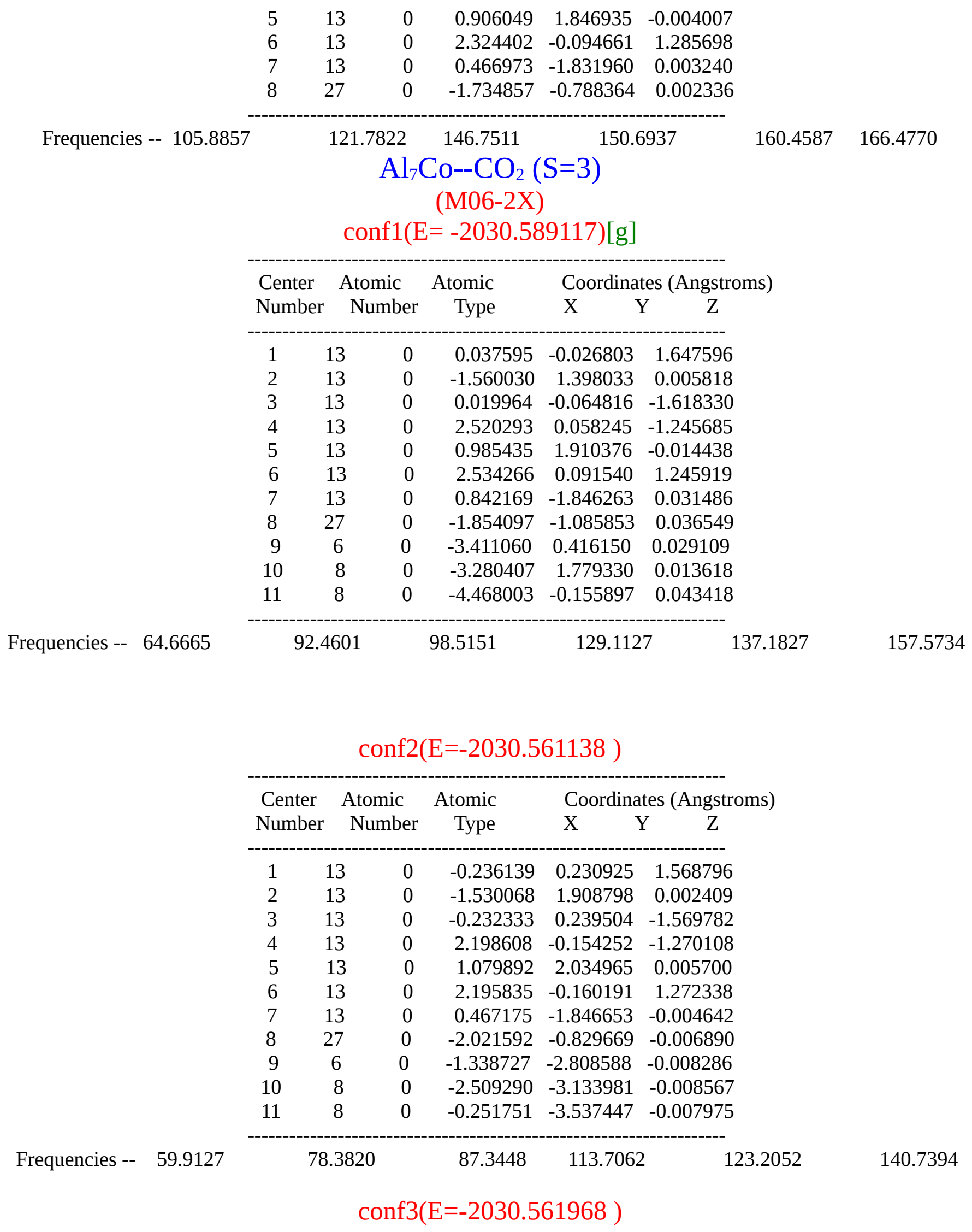




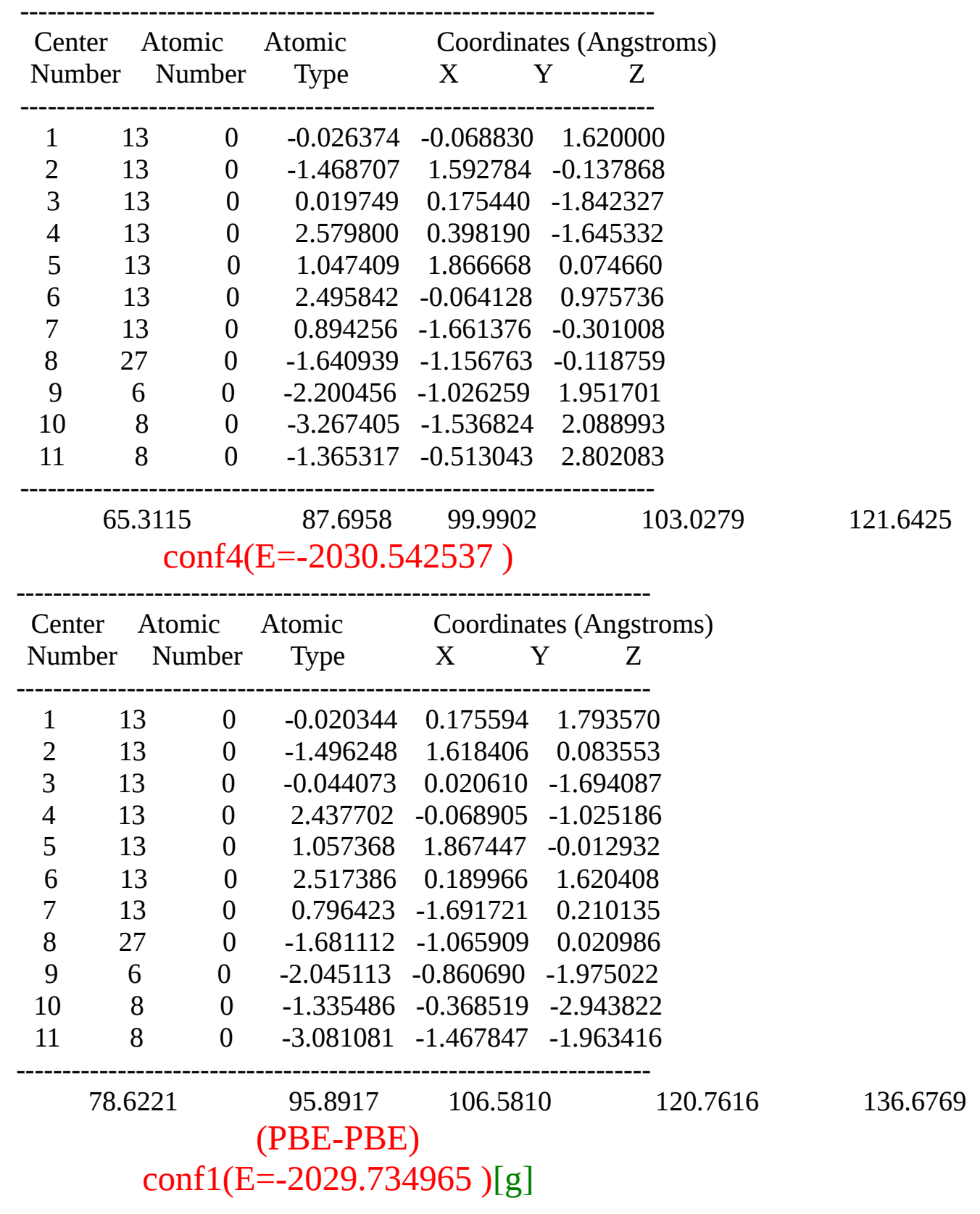

\begin{abstract}
Frequencies -- 44.2730
\end{abstract}
Frequencies --

Frequencies -- 58.2580

\begin{tabular}{|c|c|c|c|c|c|}
\hline \multirow{2}{*}{$\begin{array}{l}\text { Center } \\
\text { Number }\end{array}$} & \multirow{2}{*}{\multicolumn{2}{|c|}{$\begin{array}{l}\text { Atomic } \\
\text { Number }\end{array}$}} & \multirow{2}{*}{$\begin{array}{r}\text { Atomic } \\
\text { Type }\end{array}$} & \multicolumn{2}{|c|}{ Coordinates (Angstro } \\
\hline & & & & $\mathrm{X}$ & $\mathrm{Y} \quad \mathrm{Z}$ \\
\hline 1 & 6 & 0 & 2.836187 & 0.137645 & -0.063550 \\
\hline 2 & 8 & 0 & 2.894640 & 1.492112 & -0.085839 \\
\hline 3 & 8 & 0 & 3.780871 & -0.633628 & -0.126184 \\
\hline 4 & 13 & 0 & -0.303353 & -0.096708 & -1.594558 \\
\hline 5 & 13 & 0 & 1.073872 & 1.450055 & 0.064109 \\
\hline 6 & 13 & 0 & -0.434912 & 0.003092 & 1.740585 \\
\hline 7 & 13 & 0 & -2.949999 & -0.171190 & 1.183156 \\
\hline 8 & 13 & 0 & -1.483892 & 1.922442 & 0.037657 \\
\hline 9 & 13 & 0 & -2.867766 & 0.080919 & -1.357545 \\
\hline
\end{tabular}




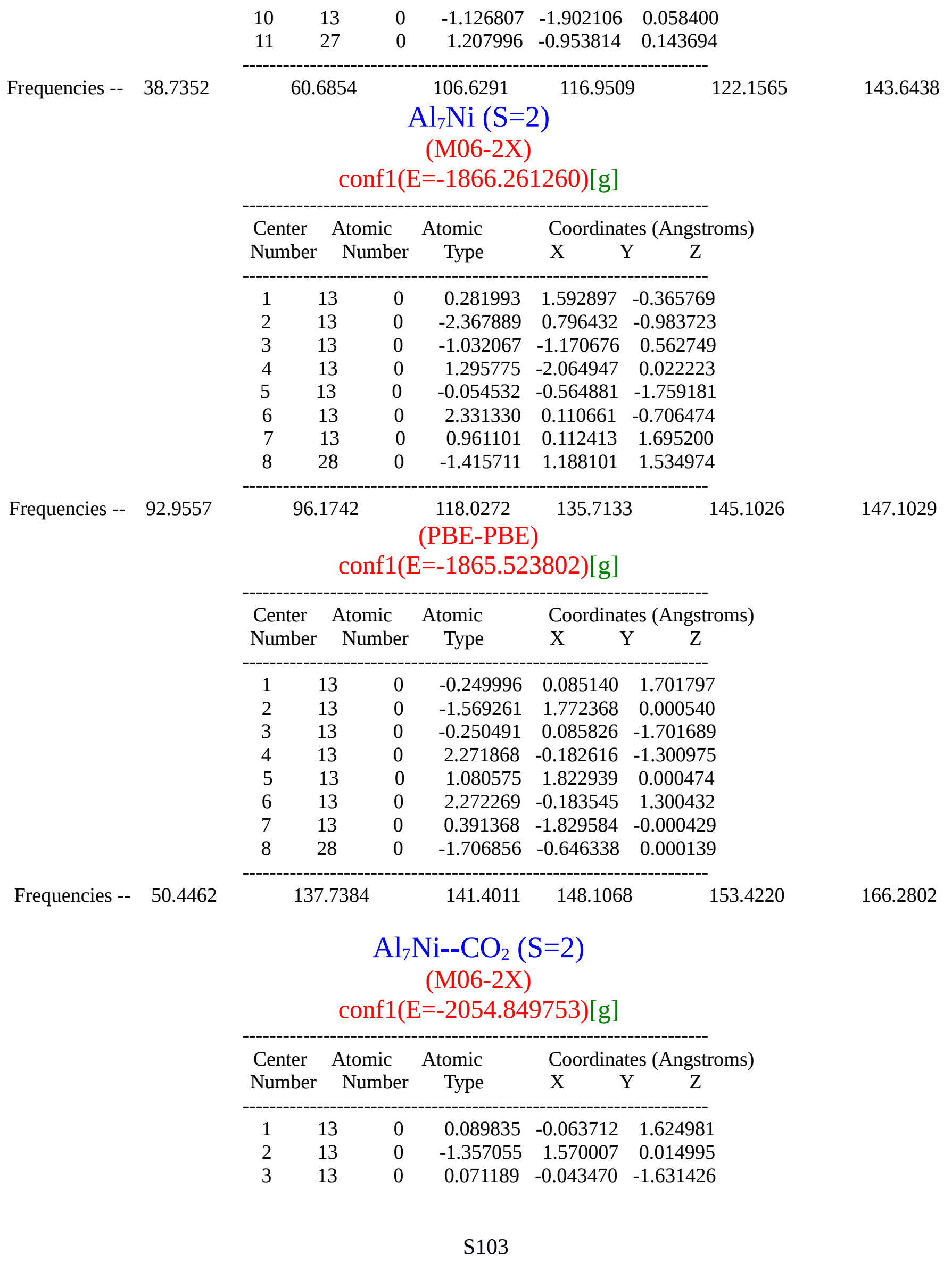




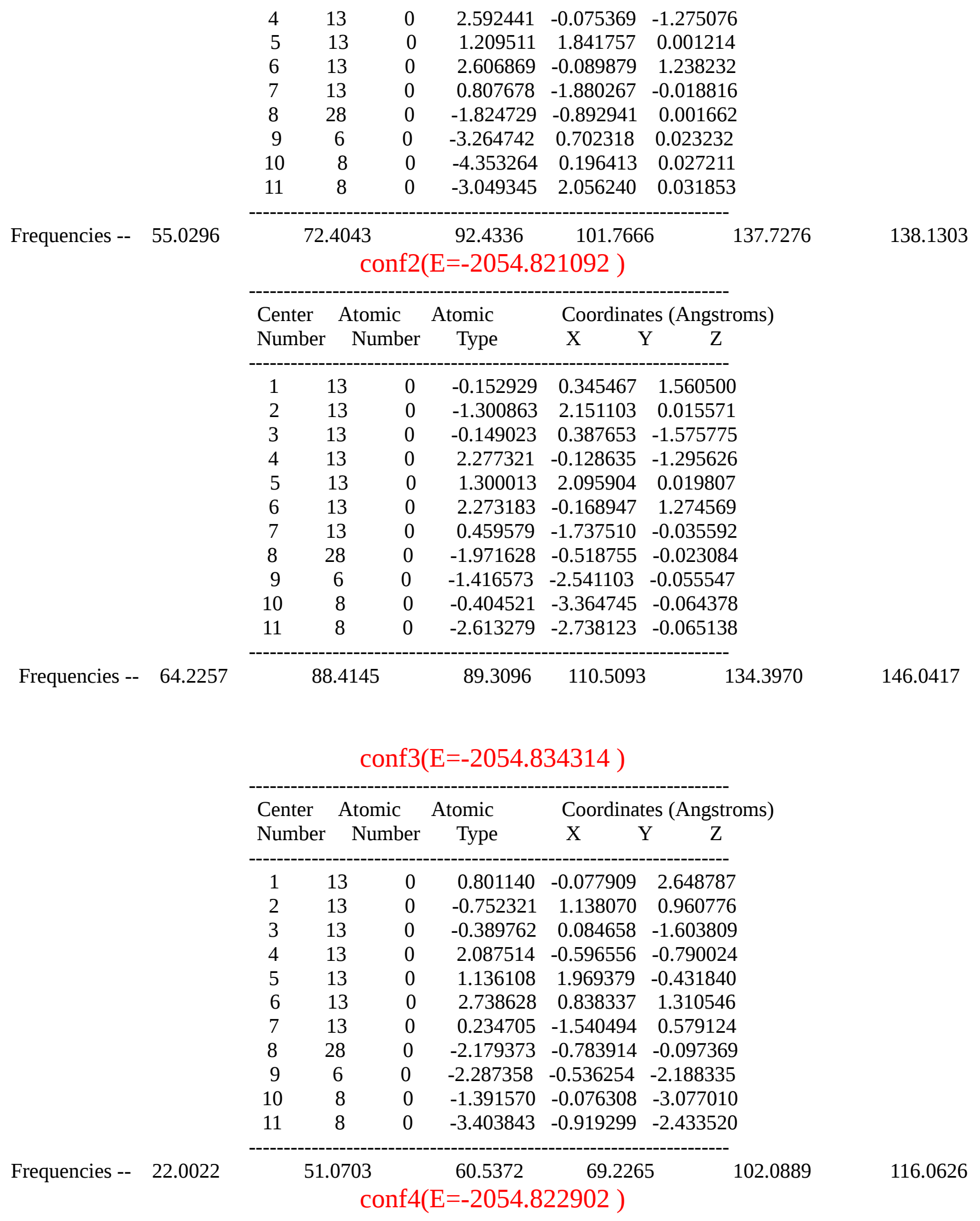




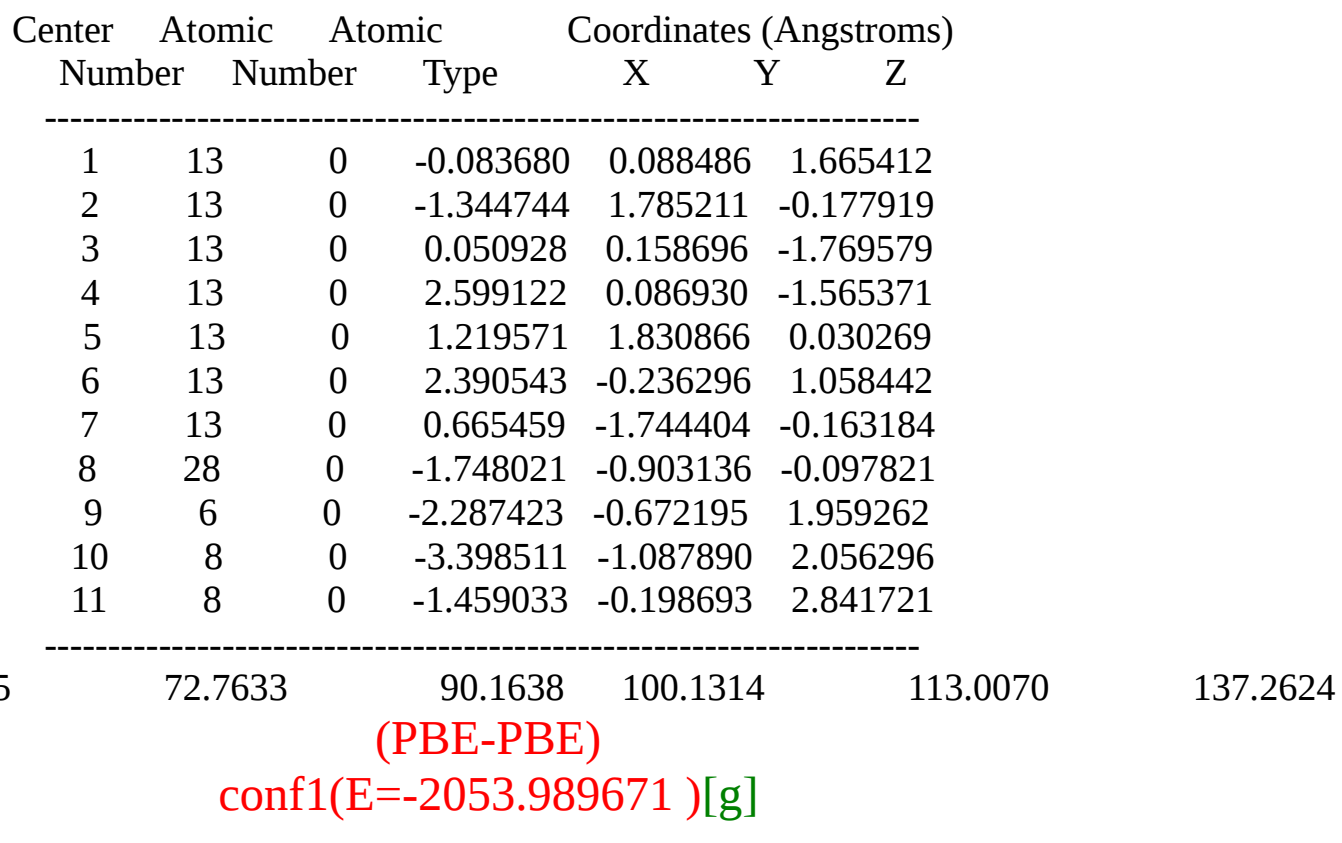

Frequencies -- 51.0455

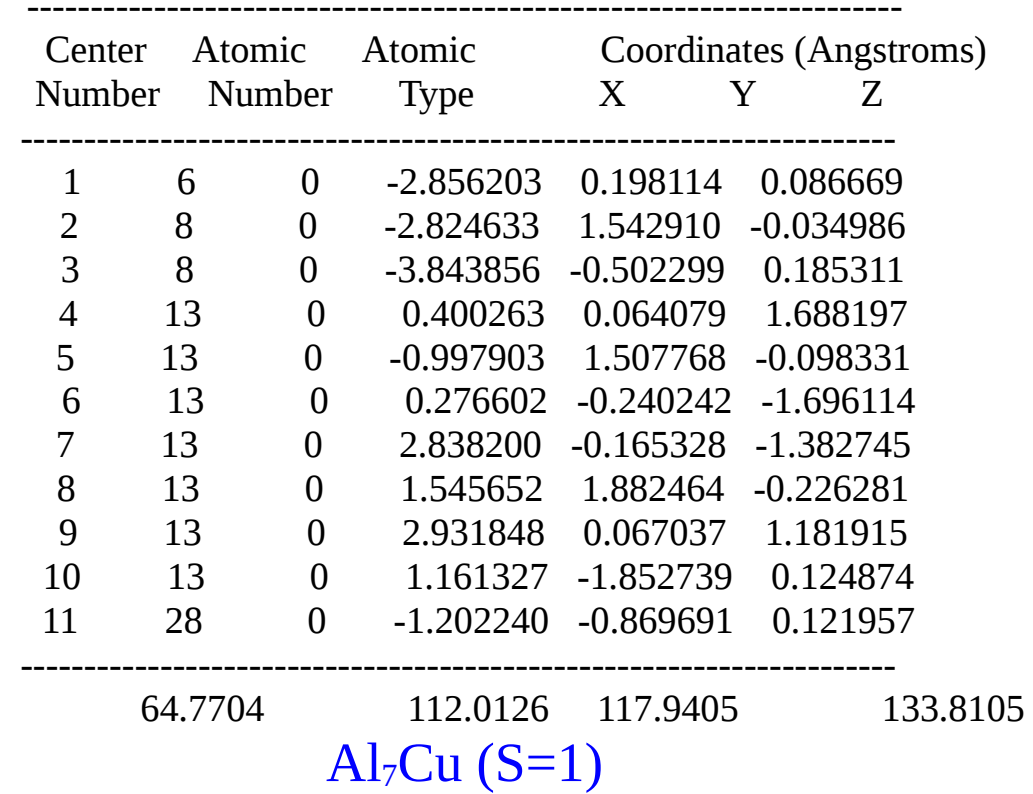

148.2263

Frequencies -- 51.0771

$$
\begin{gathered}
(\mathrm{M} 06-2 \mathrm{X}) \\
\operatorname{conf1}(\mathrm{E}=-1893.152312)[\mathrm{g}]
\end{gathered}
$$

Center Atomic Atomic Coordinates (Angstroms)

Number Number Type $\quad X \quad$ Y Z

$\begin{array}{rrrrrr} & & & & & \\ 2 & 13 & 0 & 0.217716 & -1.302379 & 1.361872 \\ 3 & 13 & 0 & -1.371107 & 1.388072 & -1.733706 \\ 4 & 13 & 0 & 1.350850 & 1.380256 & -0.910967 \\ 5 & 13 & 0 & 1.968022 & 0.792957 & 1.646922 \\ 6 & 13 & 0 & -0.444413 & 1.222560 & 0.943458 \\ 7 & 13 & 0 & 2.496248 & -0.931143 & -0.119287 \\ & & & & & \end{array}$




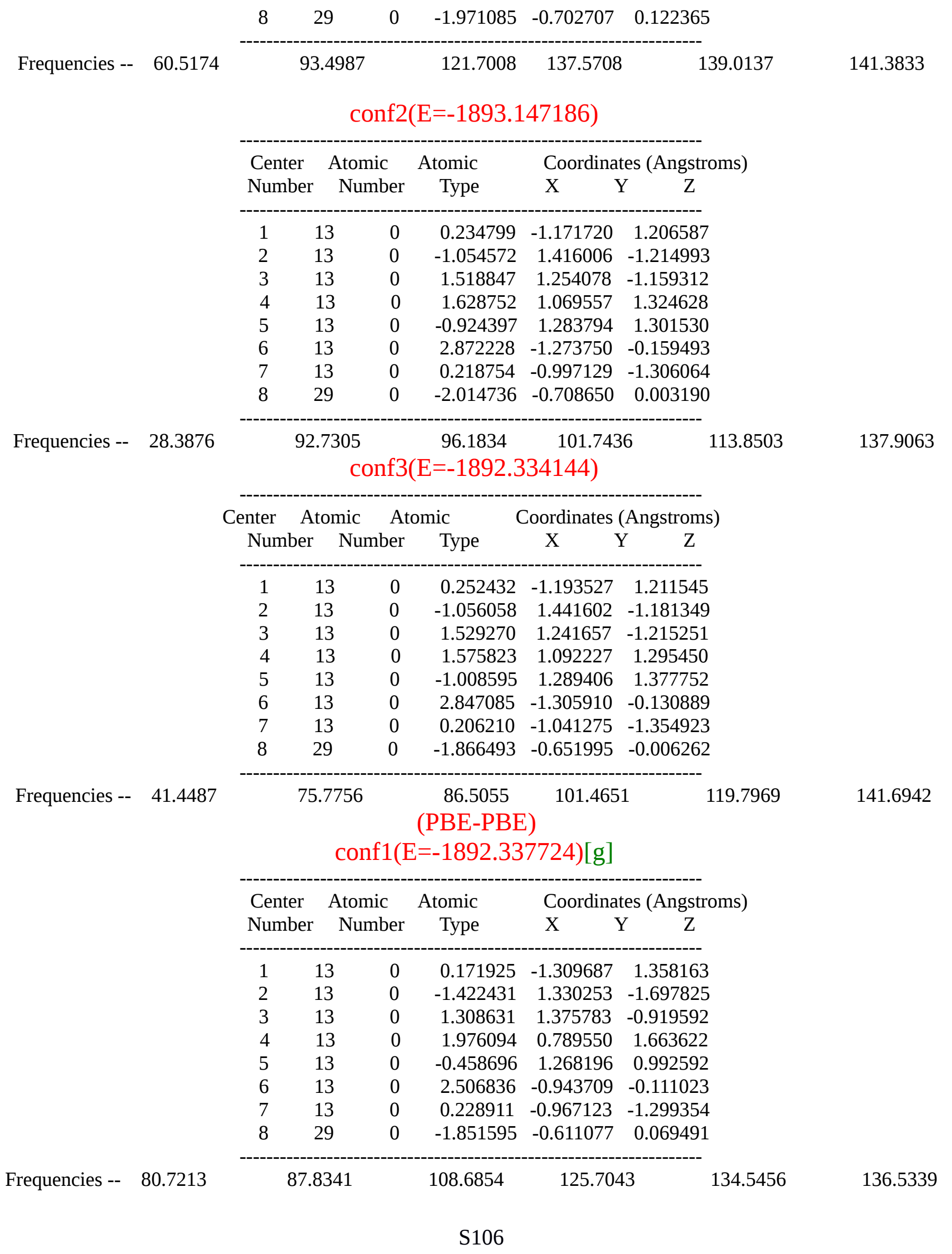




$$
\text { conf2(E=-1892.334144) }
$$

\begin{tabular}{|c|c|c|c|c|c|}
\hline \multirow{2}{*}{$\begin{array}{l}\text { Center } \\
\text { Number }\end{array}$} & \multirow{2}{*}{\multicolumn{2}{|c|}{$\begin{array}{l}\text { Atomic } \\
\text { Number }\end{array}$}} & \multirow{2}{*}{$\begin{array}{r}\text { Atomic } \\
\text { Type }\end{array}$} & \multicolumn{2}{|c|}{ Coordinates (Angstro } \\
\hline & & & & $\mathrm{X}$ & $\mathrm{Y} \quad \mathrm{Z}$ \\
\hline 1 & 13 & 0 & 0.252432 & -1.193527 & 1.211545 \\
\hline 2 & 13 & 0 & -1.0 & 1602 & -1.181349 \\
\hline 3 & 13 & 0 & 1.5 & 1.241657 & -1.215251 \\
\hline 4 & 13 & 0 & 1.575823 & 1.092227 & 1.295450 \\
\hline 5 & 13 & 0 & -1.008595 & 1.289406 & 1.377752 \\
\hline 6 & 13 & 0 & 2.847085 & -1.305910 & -0.130889 \\
\hline 7 & 13 & 0 & 0.206210 & -1.041275 & -1.354923 \\
\hline 8 & 29 & 0 & -1.866493 & -0.651995 & -0.006262 \\
\hline
\end{tabular}

Frequencies -- 41.4487

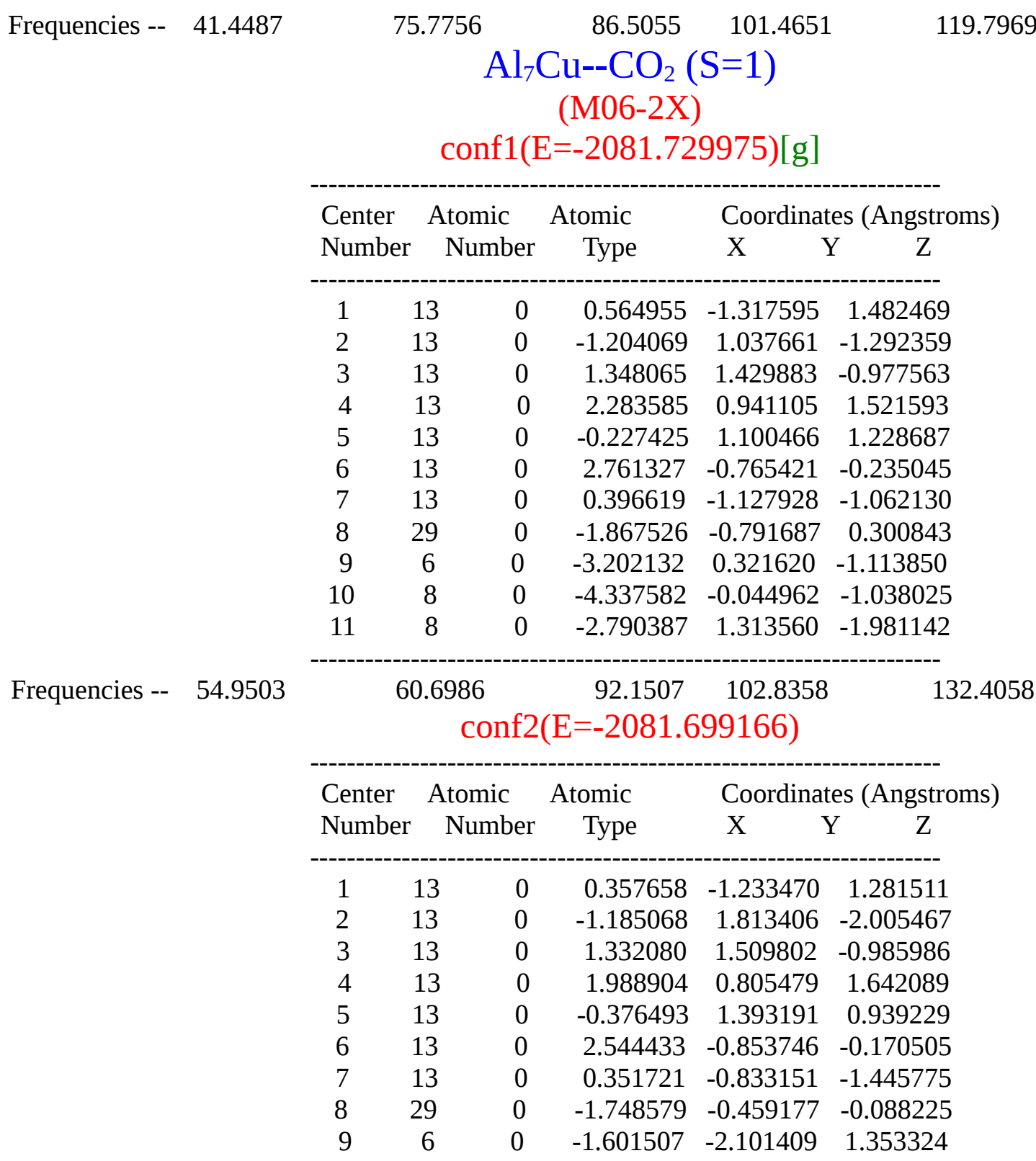

141.6942 


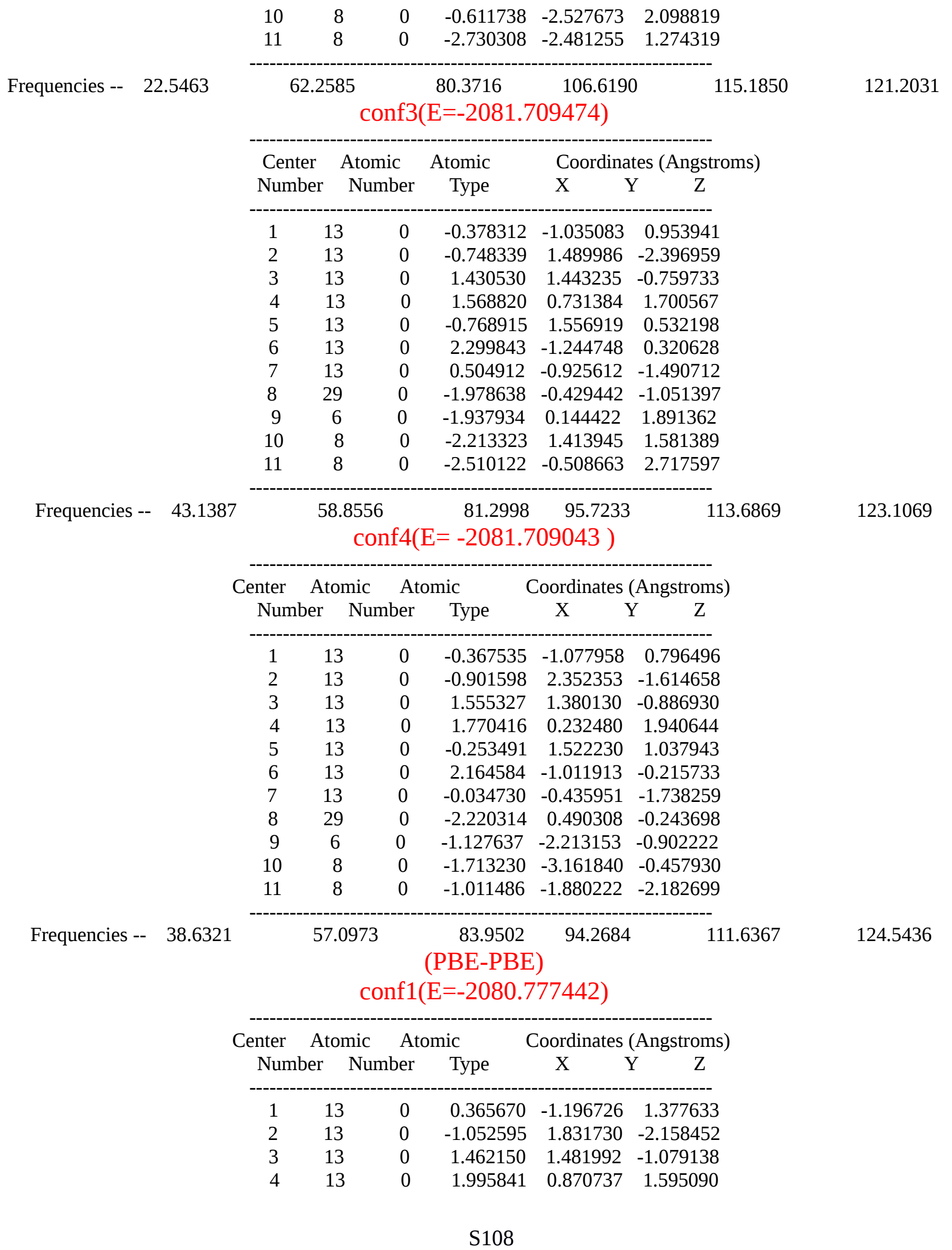




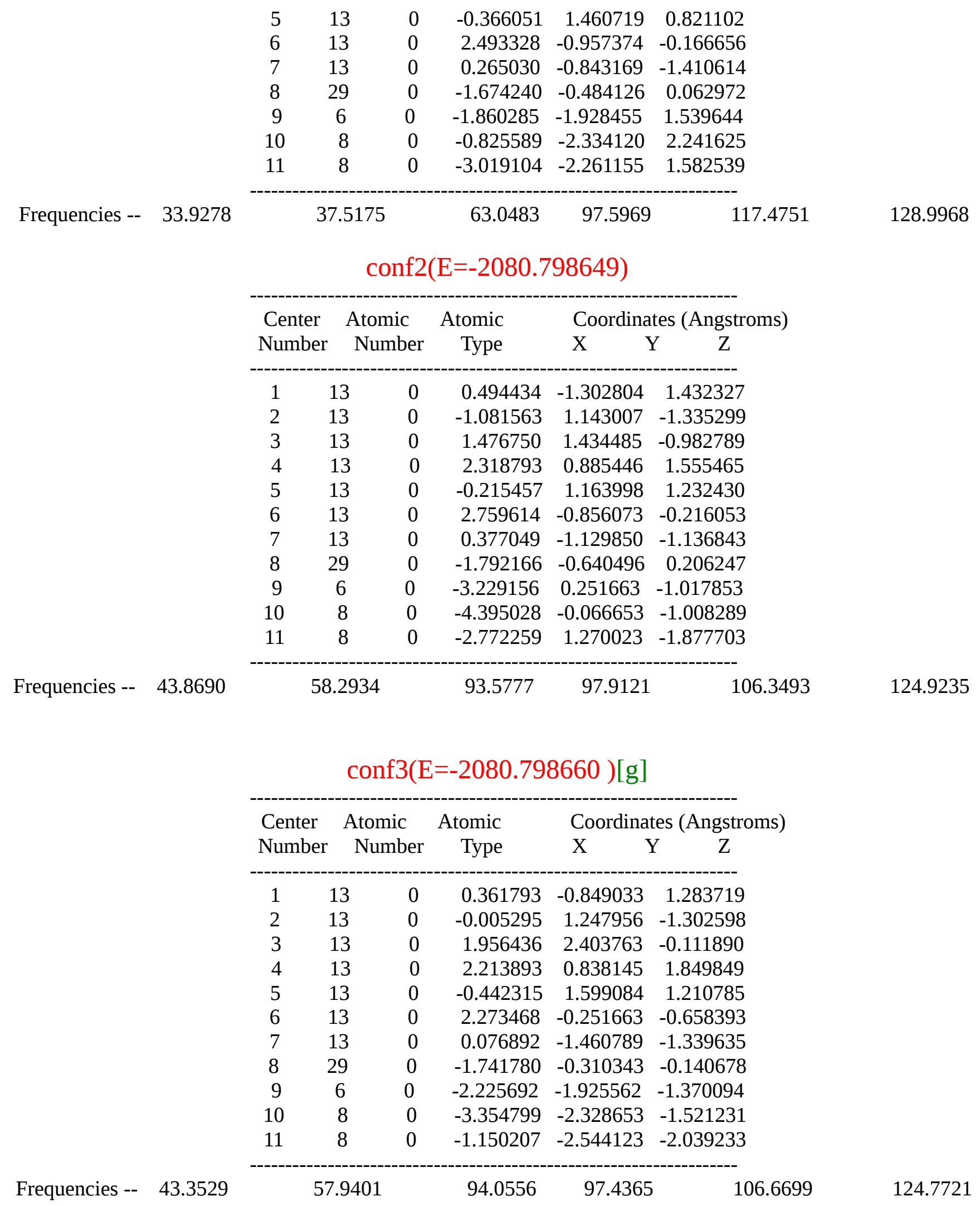




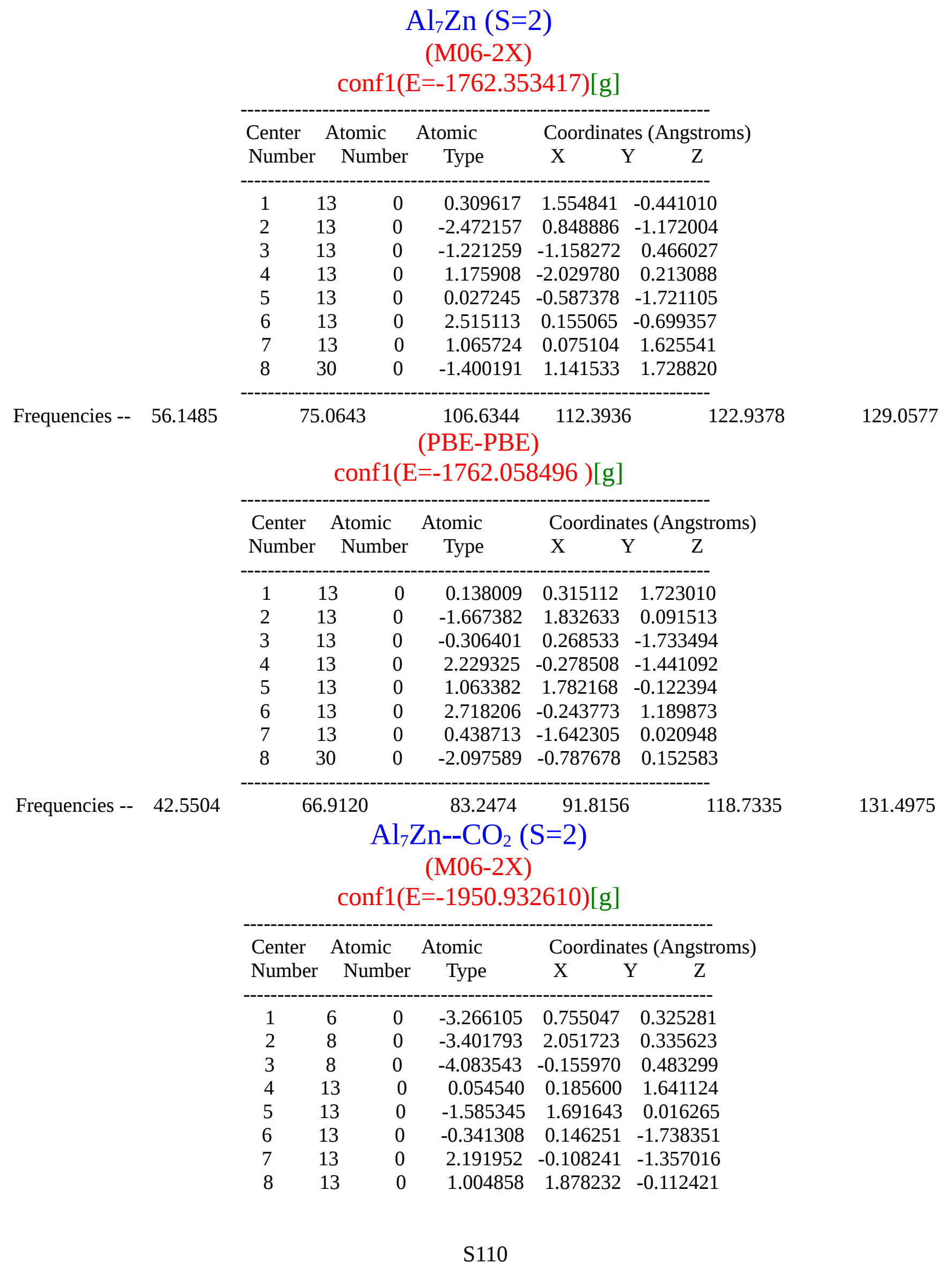




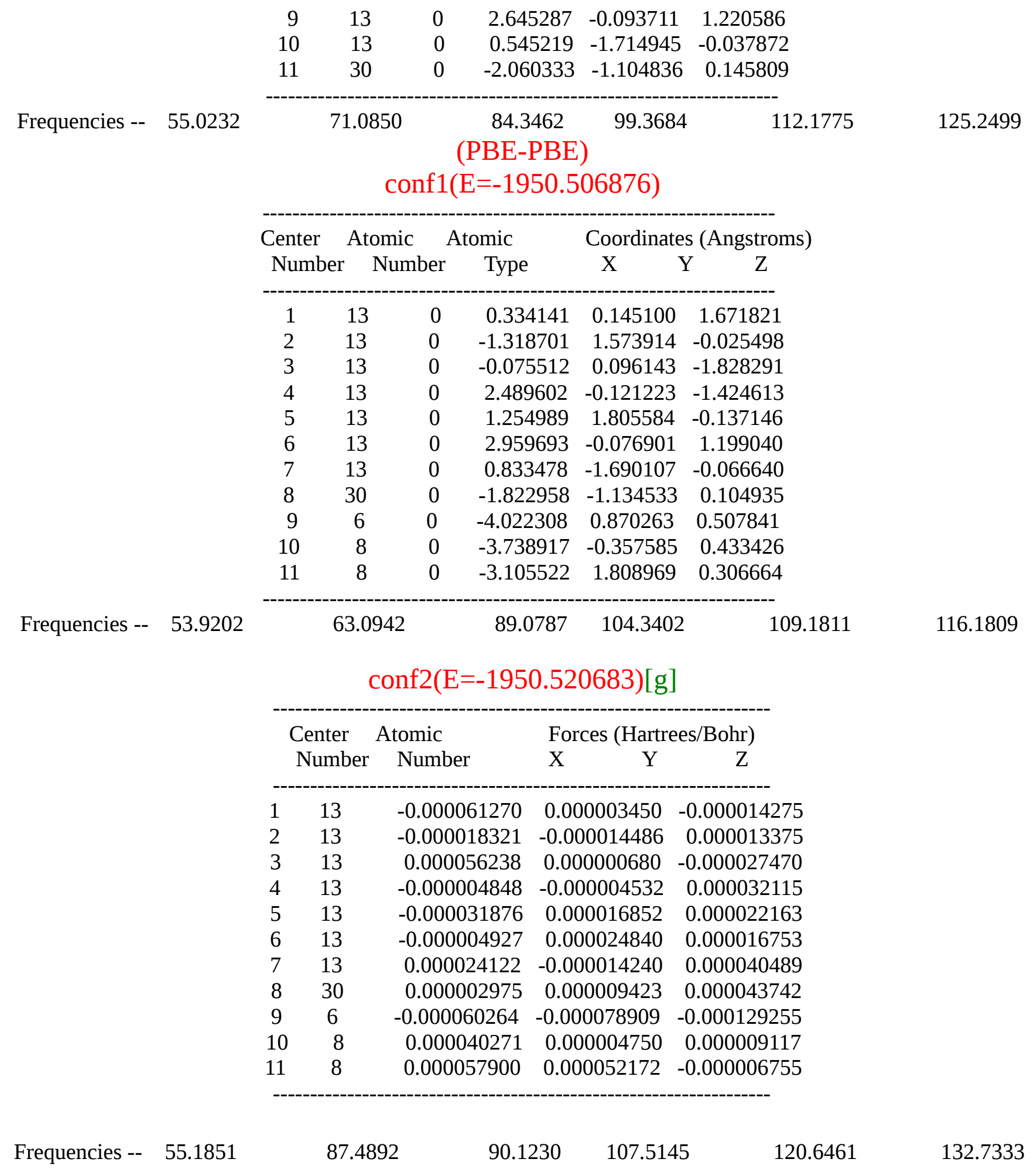


(iii) $\mathrm{Al}_{7} \mathrm{M}$ (c) cluster series

$\mathrm{Al}_{7} \mathrm{Sc}(\mathrm{S}=3)$

(M06-2X)

conf1( $E=-1743.294026)[g]$

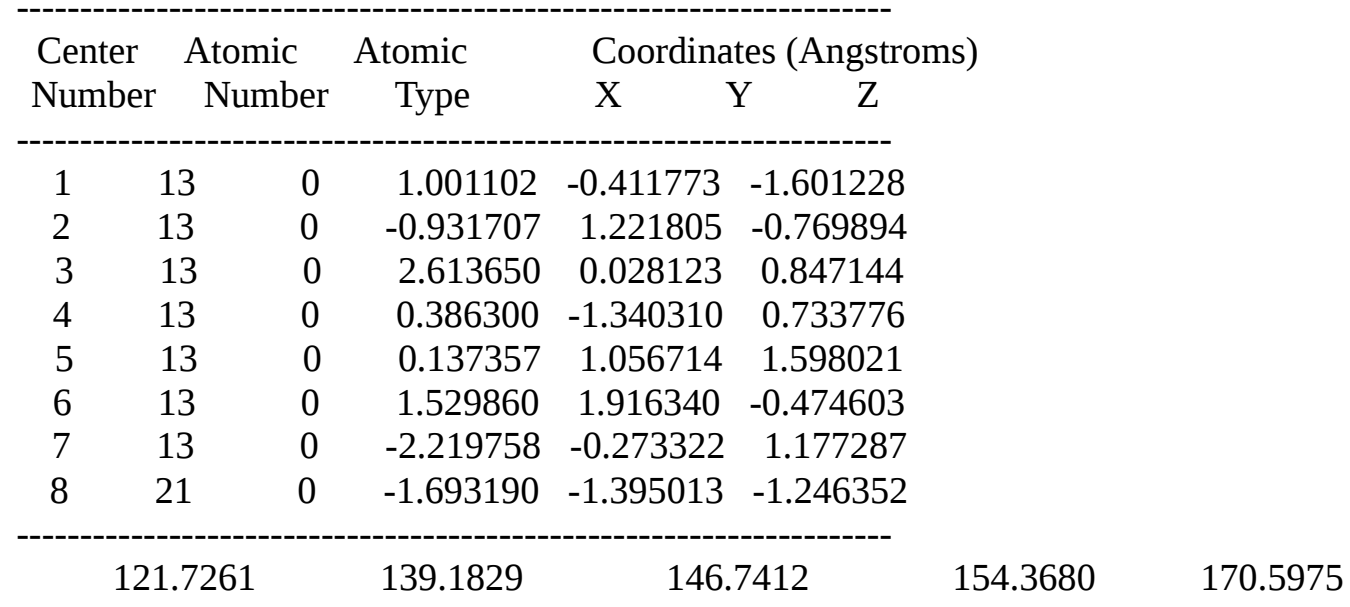

Frequencies - $\quad 97.4729$

(PBE-PBE)

conf1(E=-1742.591663 )[g]

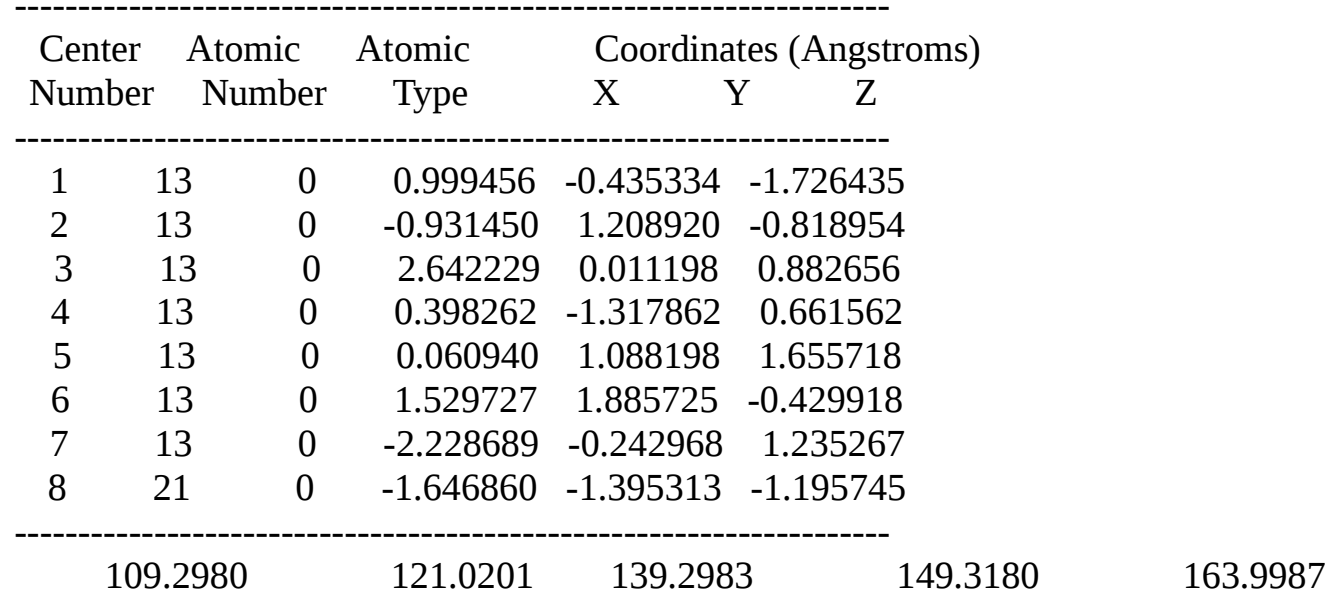

Frequencies -- 62.2017

\begin{tabular}{|c|c|c|c|c|c|}
\hline \multirow{2}{*}{$\begin{array}{l}\text { Center } \\
\text { Number }\end{array}$} & \multirow{2}{*}{\multicolumn{2}{|c|}{$\begin{array}{l}\text { Atomic } \\
\text { Number }\end{array}$}} & \multirow{2}{*}{$\begin{array}{r}\text { Atomic } \\
\text { Type }\end{array}$} & \multicolumn{2}{|c|}{ Coordinates (Angstroms) } \\
\hline & & & & $\mathrm{X} \quad \mathrm{Y}$ & $\mathrm{Y} \quad \mathrm{Z}$ \\
\hline 1 & 13 & 0 & 1.268858 & -0.529141 & -1.423648 \\
\hline 2 & 13 & 0 & -0.737378 & 1.267972 & -0.949682 \\
\hline 3 & 13 & 0 & 2.675069 & -0.006099 & 1.495609 \\
\hline 4 & 13 & 0 & 0.585139 & -1.446532 & 0.869669 \\
\hline 5 & 13 & 0 & 0.178112 & 1.187154 & 1.568552 \\
\hline
\end{tabular}




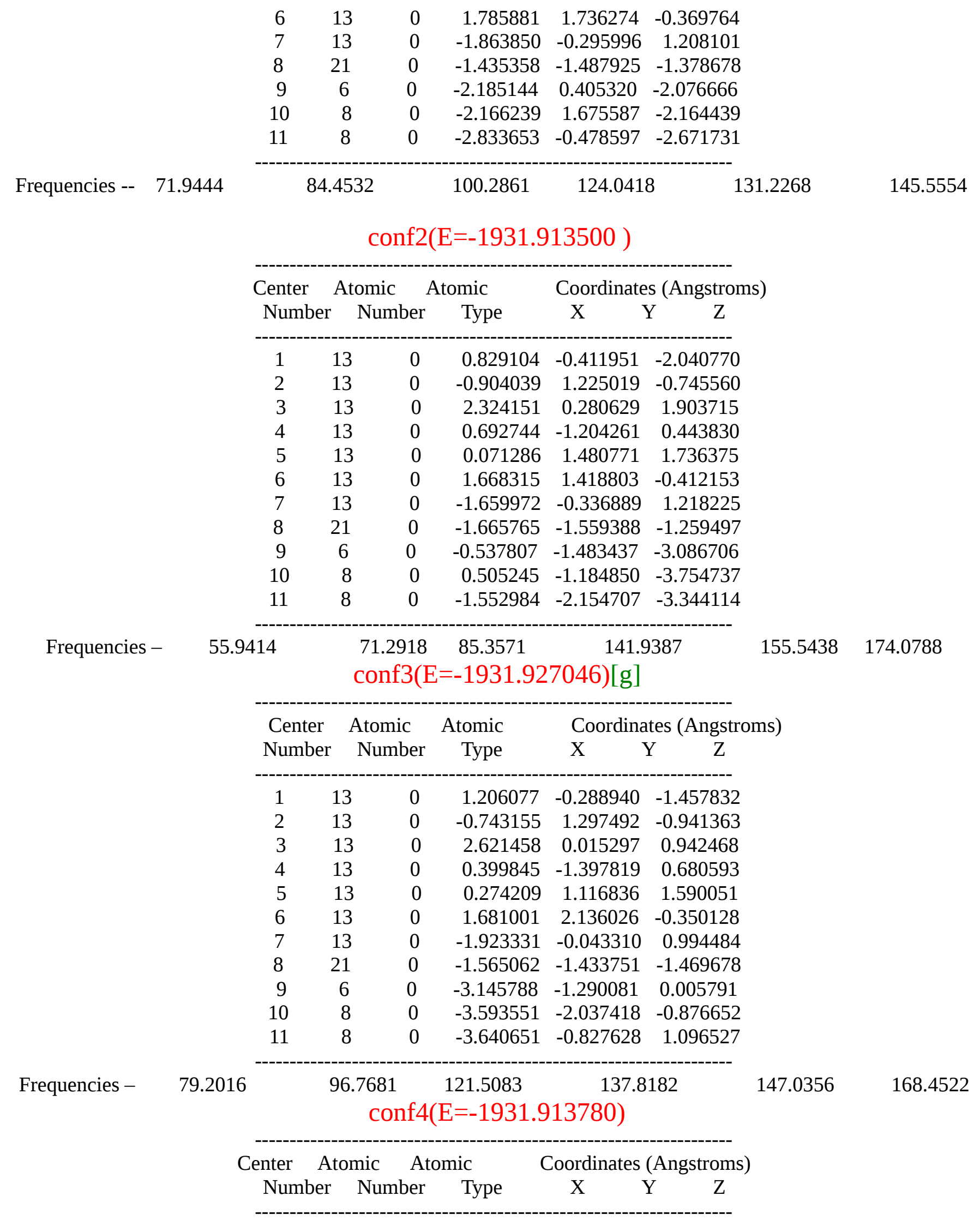




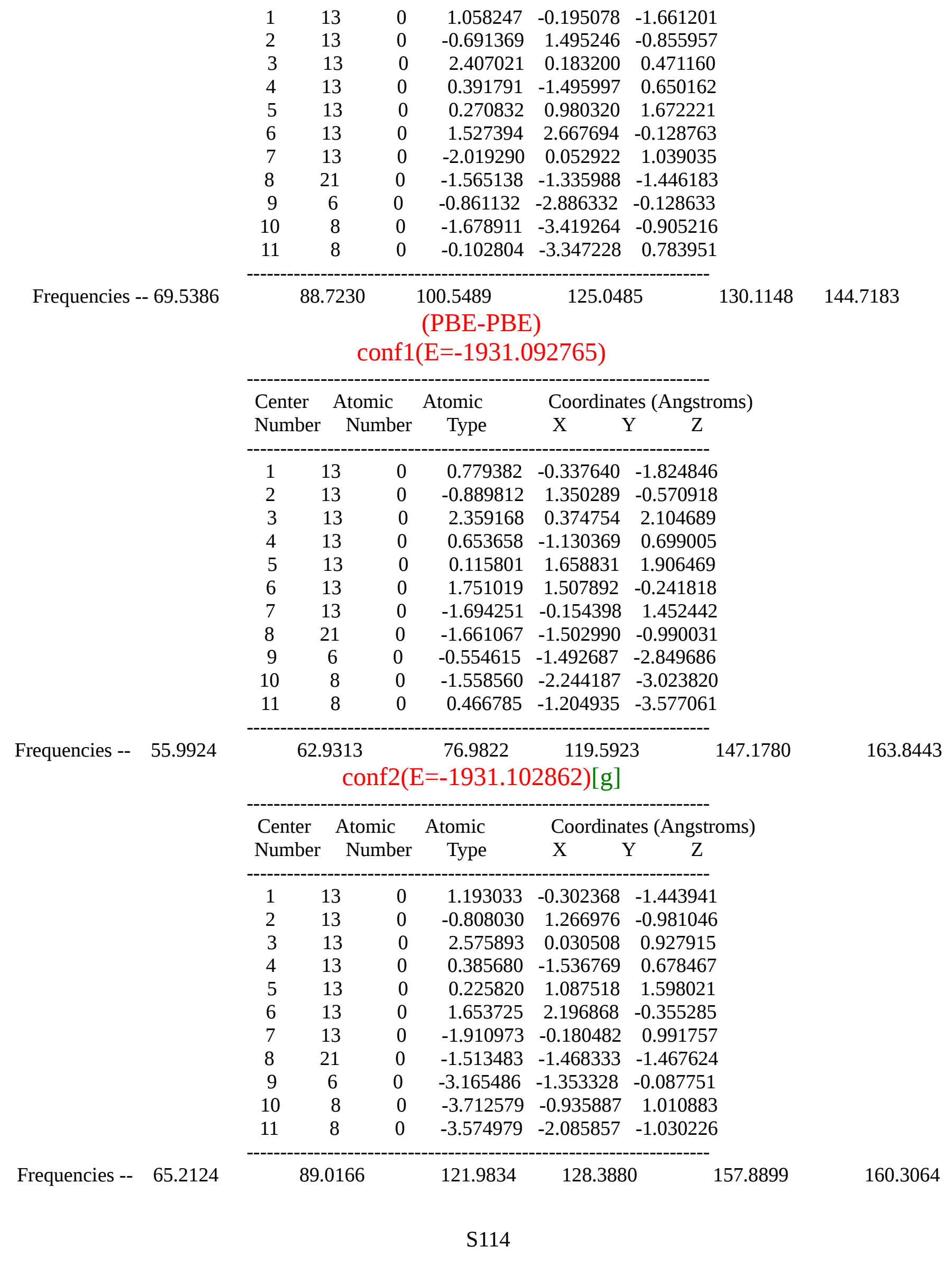




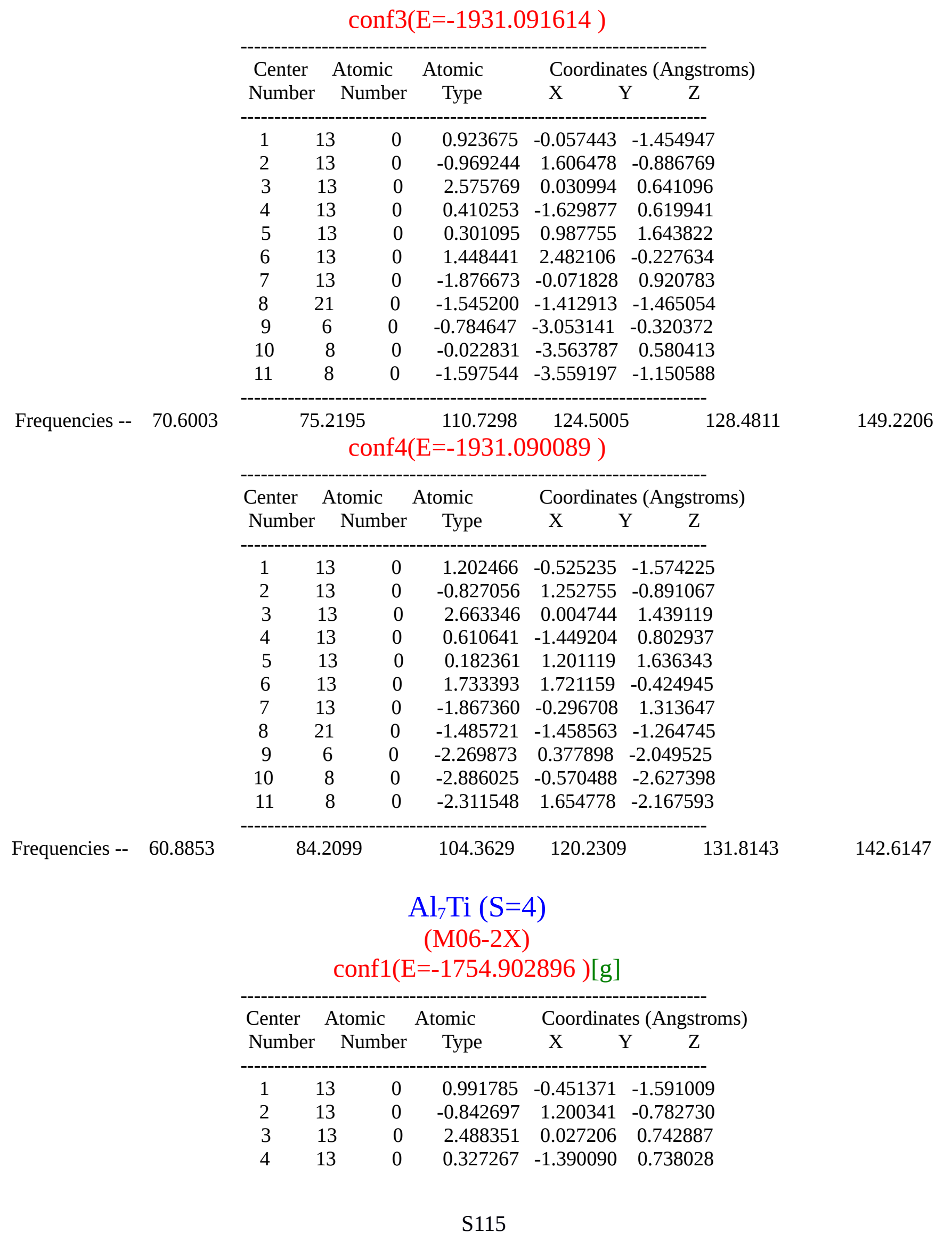




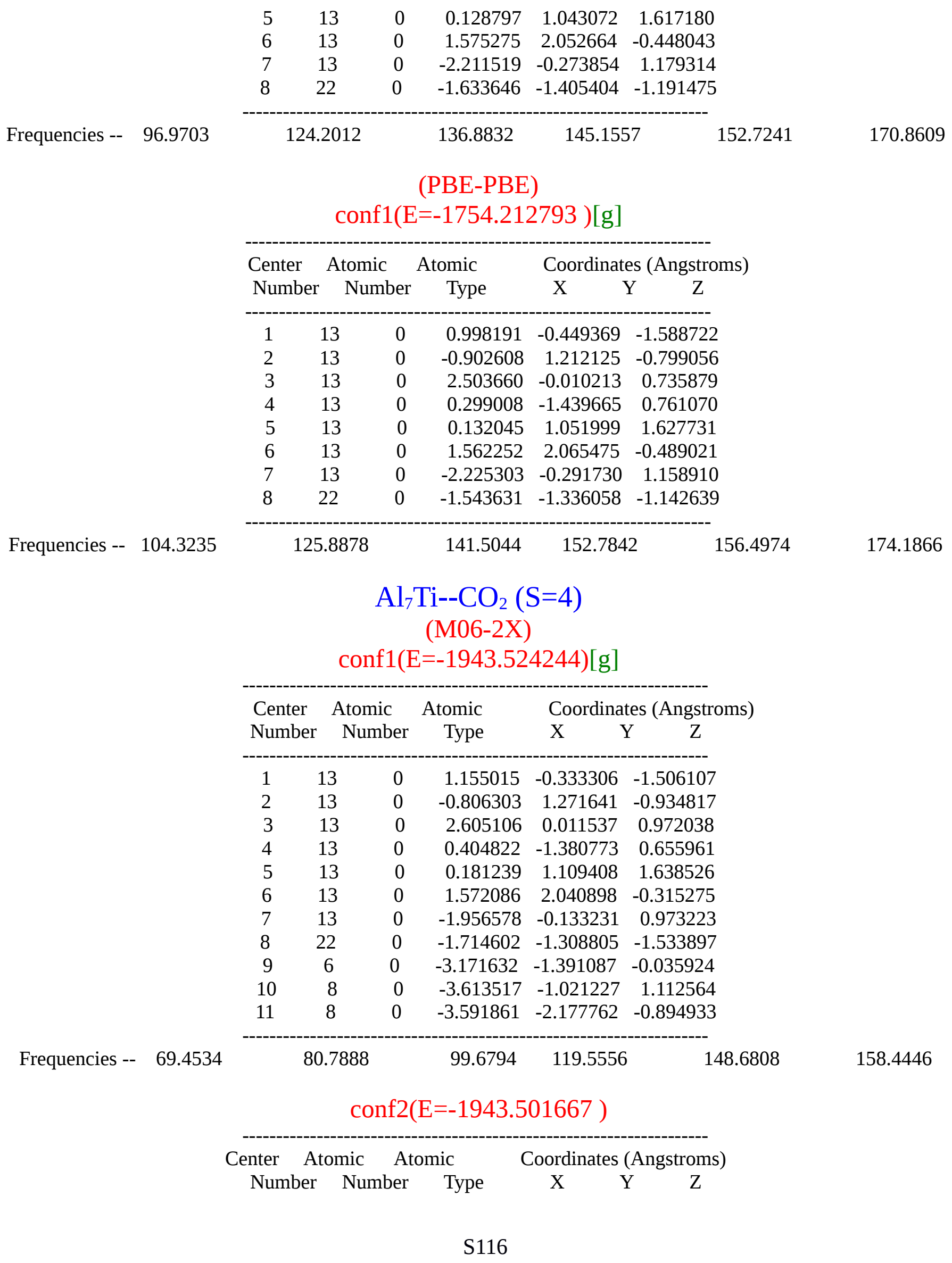




$\begin{array}{rrrrrr}1 & 13 & 0 & 0.935540 & -0.384062 & -1.545904 \\ 2 & 13 & 0 & -0.872626 & 1.256446 & -0.466609 \\ 3 & 13 & 0 & 2.431272 & -0.021772 & 0.666590 \\ 4 & 13 & 0 & 0.243293 & -1.200731 & 0.896098 \\ 5 & 13 & 0 & 0.230204 & 1.208752 & 1.899260 \\ 6 & 13 & 0 & 1.505357 & 2.015727 & -0.461962 \\ 7 & 13 & 0 & -2.148926 & -0.097820 & 1.490227 \\ 8 & 22 & 0 & -1.840929 & -1.392732 & -1.093695 \\ 9 & 6 & 0 & -0.350834 & -1.549557 & -2.612789 \\ 10 & 8 & 0 & -1.349194 & -2.195494 & -2.974414 \\ 11 & 8 & 0 & 0.779887 & -1.343889 & -3.167530 \\ ----------------------------------------------------------\end{array}$

Frequencies -- 87.7843

98.3423

123.5695

124.9088

148.3205

156.7316

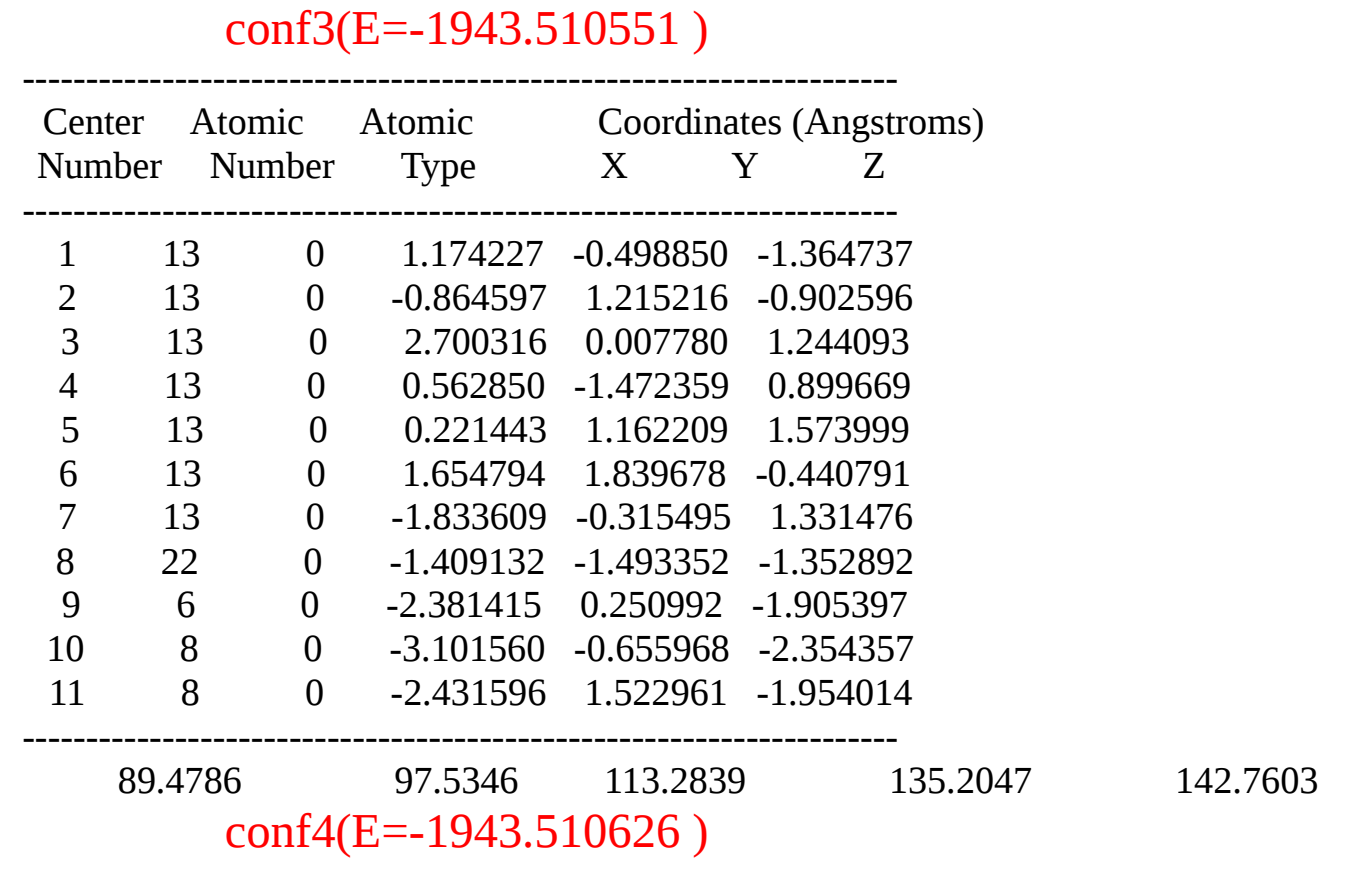

\begin{tabular}{|c|c|c|c|c|c|}
\hline \multirow{2}{*}{$\begin{array}{l}\text { Center } \\
\text { Number }\end{array}$} & \multirow{2}{*}{\multicolumn{2}{|c|}{$\begin{array}{l}\text { Atomic } \\
\text { Number }\end{array}$}} & \multirow{2}{*}{$\begin{array}{r}\text { Atomic } \\
\text { Type }\end{array}$} & \multicolumn{2}{|c|}{ Coordinates (Angstro } \\
\hline & & & & $\mathrm{X}$ & Y $\quad Z$ \\
\hline 1 & 13 & 0 & 1.009738 & -0.185234 & -1.555015 \\
\hline 2 & 13 & 0 & -0.762392 & 1.490247 & -0.844185 \\
\hline 3 & 13 & 0 & 2.413217 & 0.116808 & 0.566112 \\
\hline 4 & 13 & 0 & 0.331334 & -1.491997 & 0.713523 \\
\hline 5 & 13 & 0 & 0.280852 & 1.047332 & 1.648870 \\
\hline 6 & 13 & 0 & 1.573135 & 2.541572 & -0.273258 \\
\hline 7 & 13 & 0 & -2.020445 & 0.112636 & 1.104831 \\
\hline 8 & 22 & 0 & -1.494230 & -1.353809 & -1.405658 \\
\hline 9 & 6 & 0 & -1.022483 & -2.844640 & -0.043902 \\
\hline 10 & 8 & 0 & -0.307114 & -3.282725 & 0.914737 \\
\hline 11 & 8 & 0 & -1.932224 & -3.337198 & -0.731085 \\
\hline
\end{tabular}




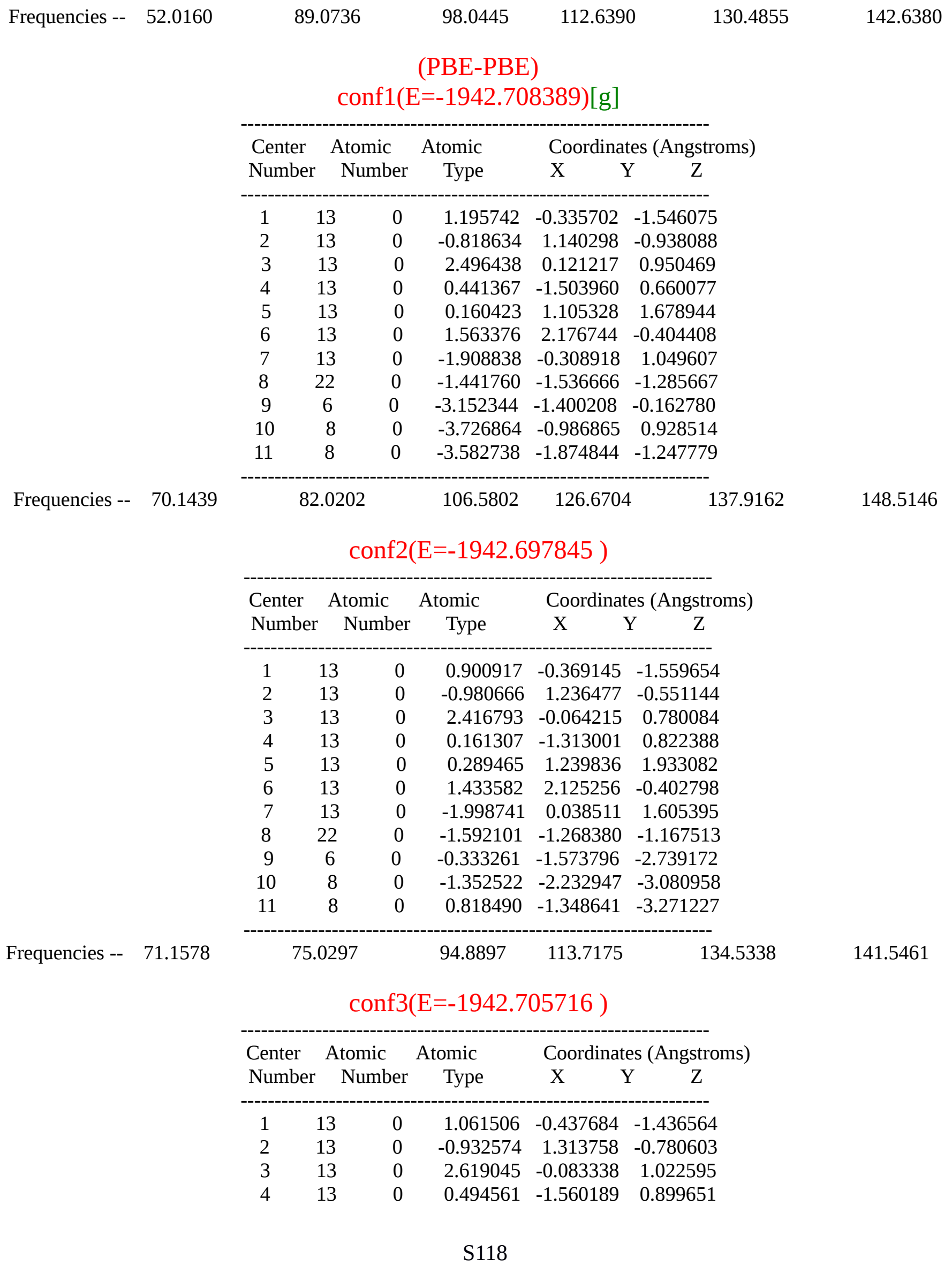




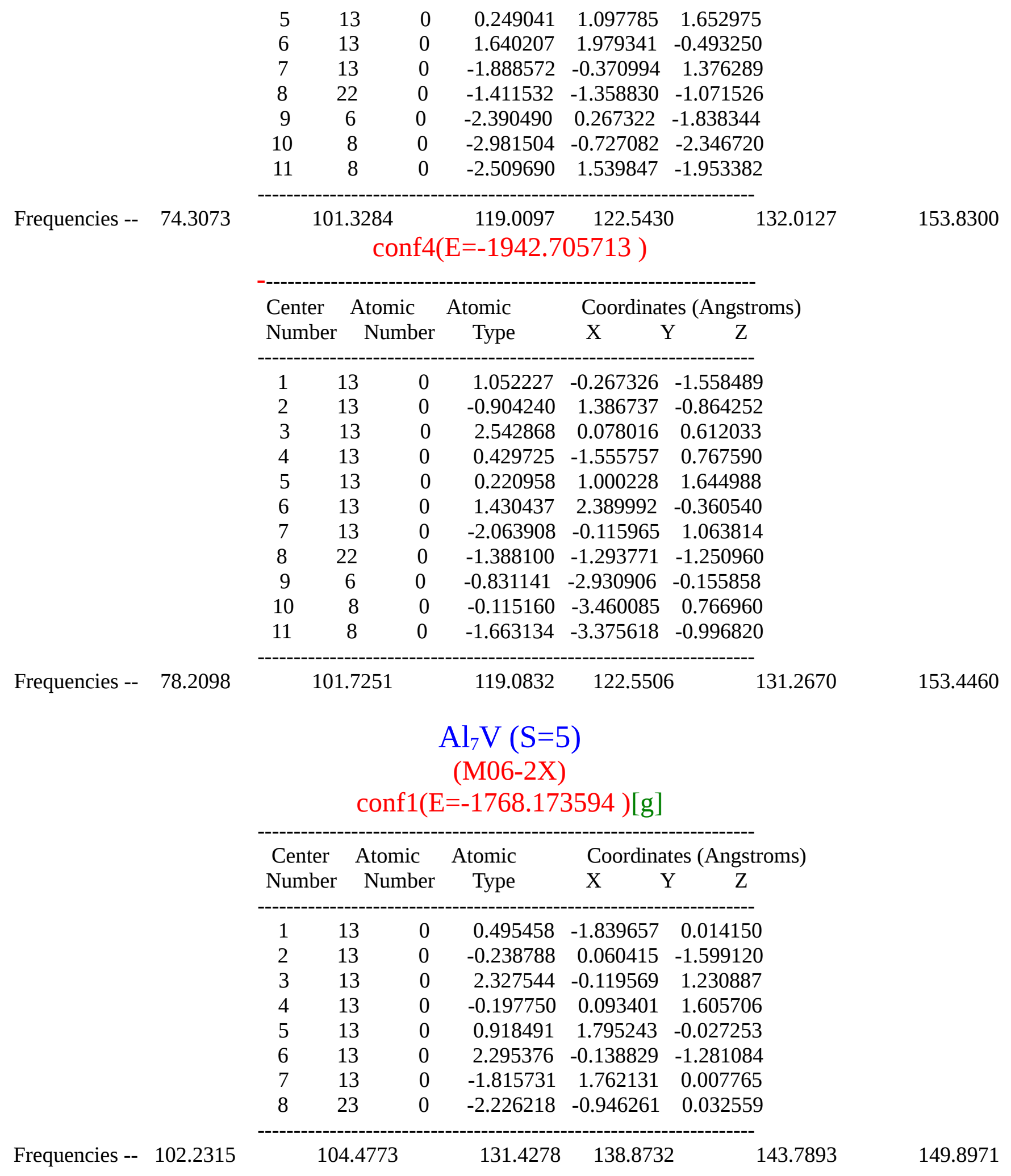




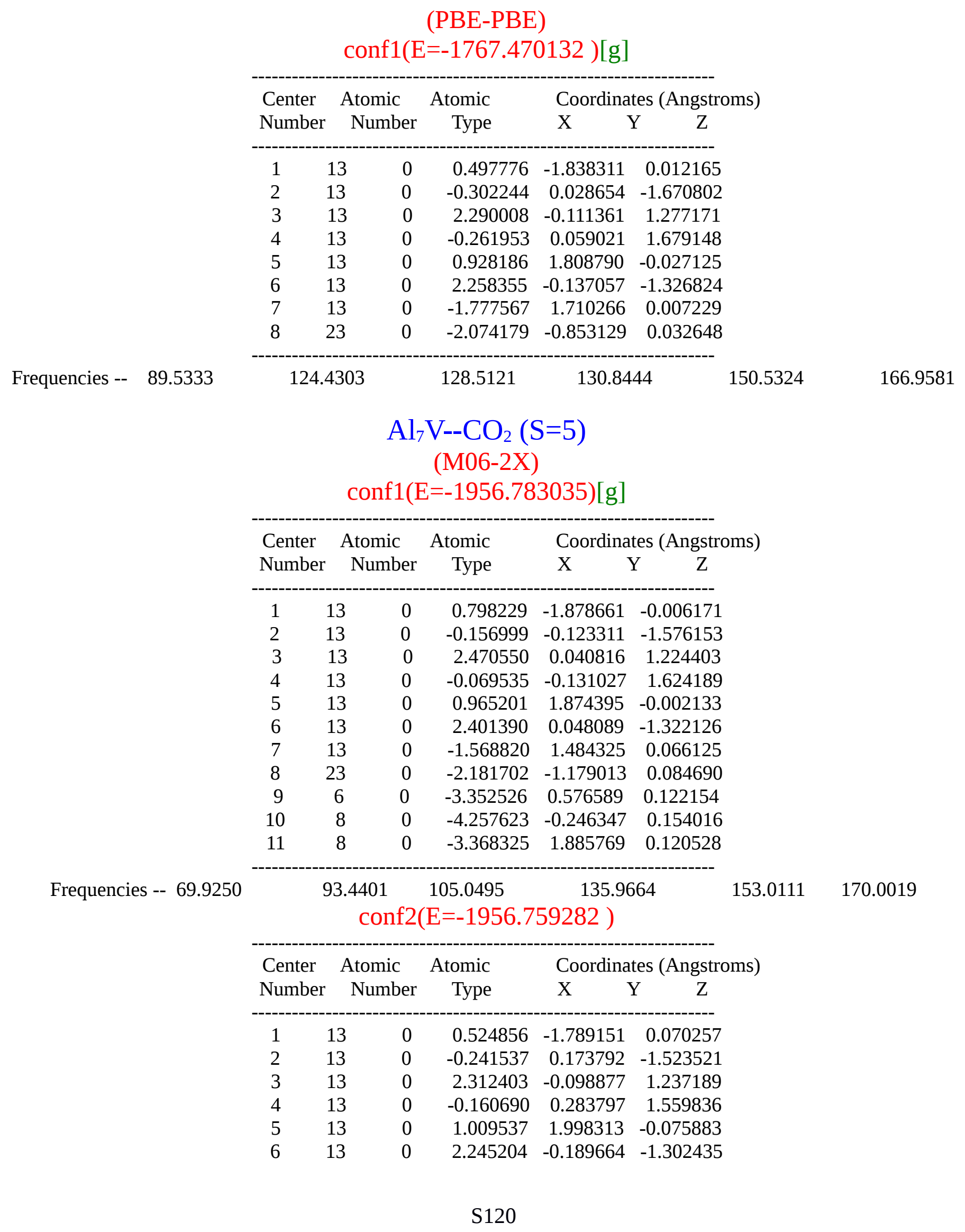




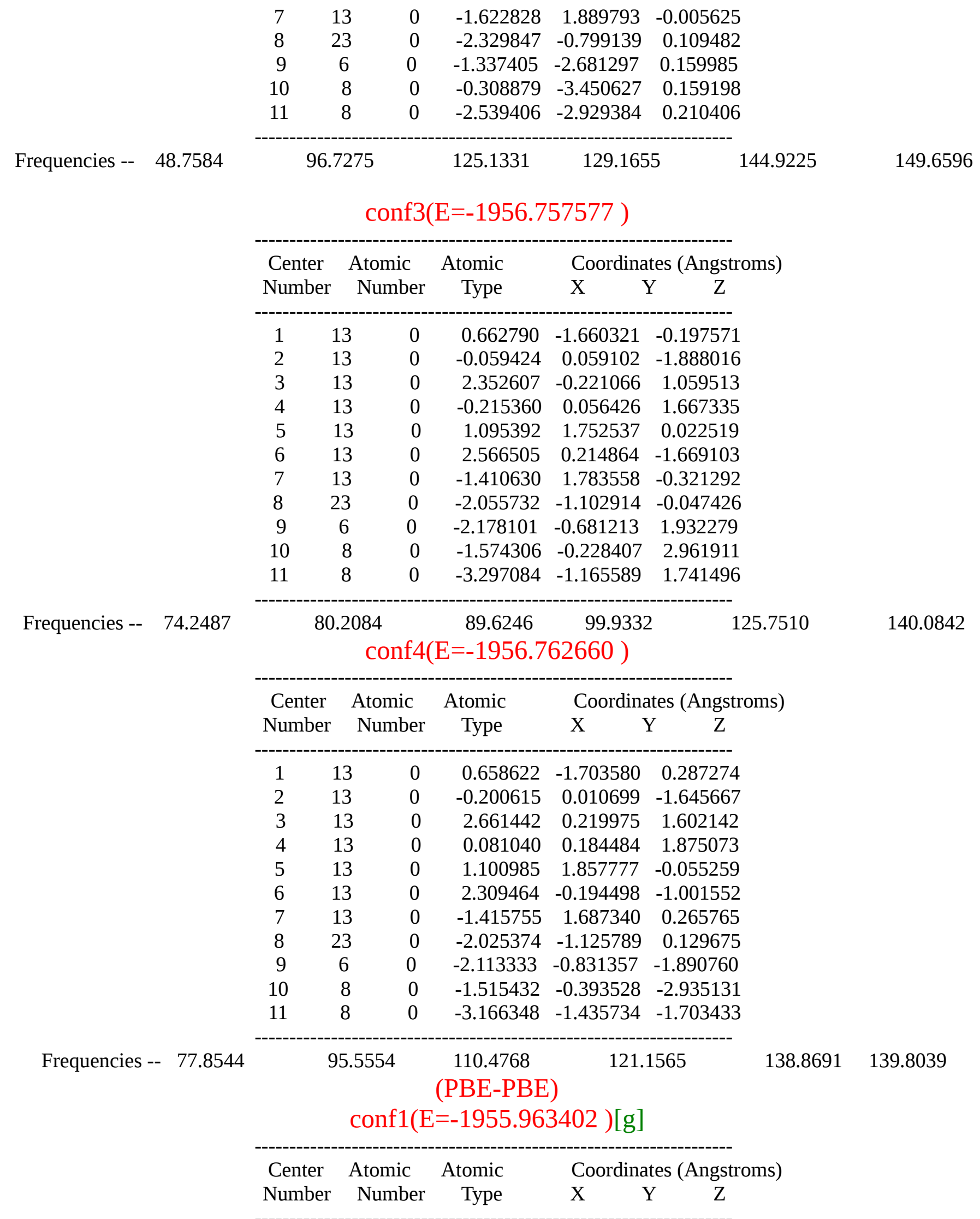




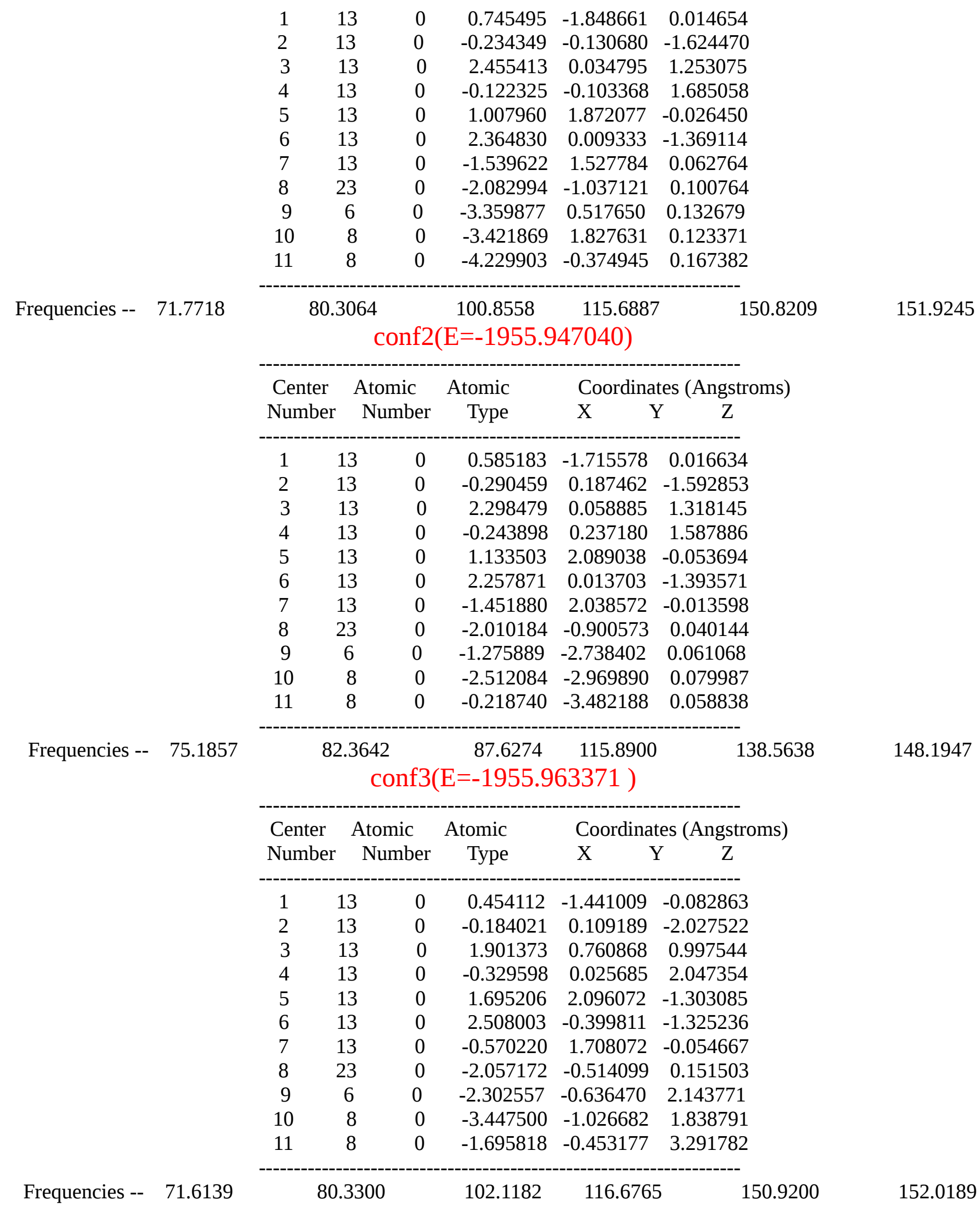




\section{conf4(E=-1955.963374 )}

\begin{tabular}{|c|c|c|c|c|c|}
\hline \multirow{2}{*}{$\begin{array}{l}\text { Center } \\
\text { Number }\end{array}$} & \multirow{2}{*}{\multicolumn{2}{|c|}{$\begin{array}{l}\text { Atomic } \\
\text { Number }\end{array}$}} & \multirow{2}{*}{$\begin{array}{r}\text { Atomic } \\
\text { Type }\end{array}$} & \multicolumn{2}{|c|}{ Coordinates (Angstroms) } \\
\hline & & & & $\mathrm{X} \quad \mathrm{Y}$ & $\mathrm{Y} \quad \mathrm{Z}$ \\
\hline 1 & 13 & 0 & 0.444503 & -1.485454 & 0.130113 \\
\hline 2 & 13 & 0 & -0.429306 & -0.172468 & -2.060841 \\
\hline 3 & 13 & 0 & 2.515080 & -0.332817 & 1.238899 \\
\hline 4 & 13 & 0 & -0.161907 & 0.174535 & 1.991331 \\
\hline 5 & 13 & 0 & 1.653213 & 2.141901 & 1.084719 \\
\hline 6 & 13 & 0 & 1.816425 & 0.668908 & -1.132207 \\
\hline 7 & 13 & 0 & -0.642304 & 1.636288 & -0.063934 \\
\hline 8 & 23 & 0 & -2.093255 & -0.625504 & -0.085549 \\
\hline 9 & 6 & 0 & -2.392061 & -0.869895 & -2.059897 \\
\hline 10 & 8 & 0 & -1.820360 & -0.746383 & -3.233747 \\
\hline 11 & 8 & 0 & -3.522471 & -1.254528 & -1.700858 \\
\hline
\end{tabular}

151.8432

Frequencies -- 71.6705

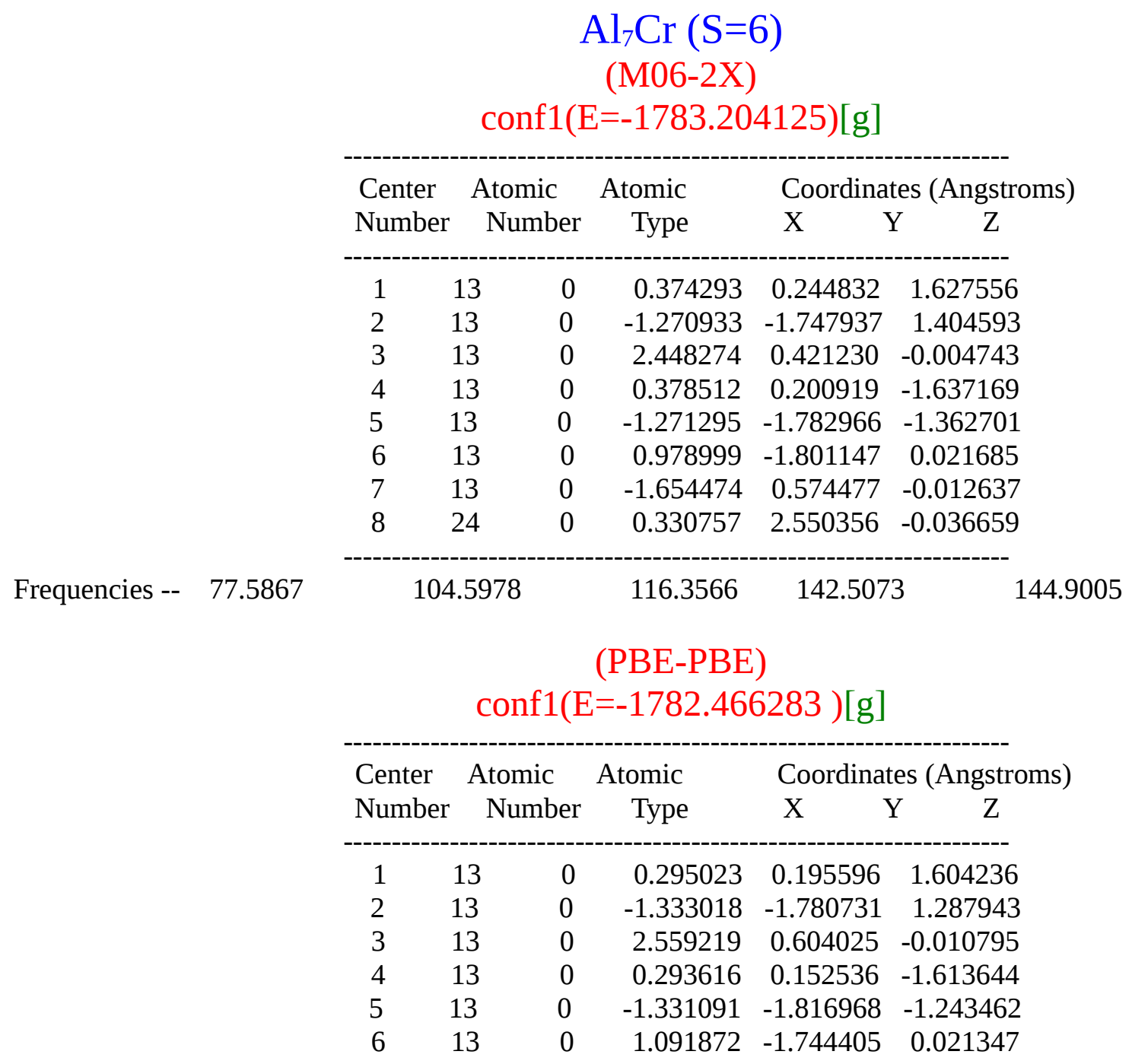




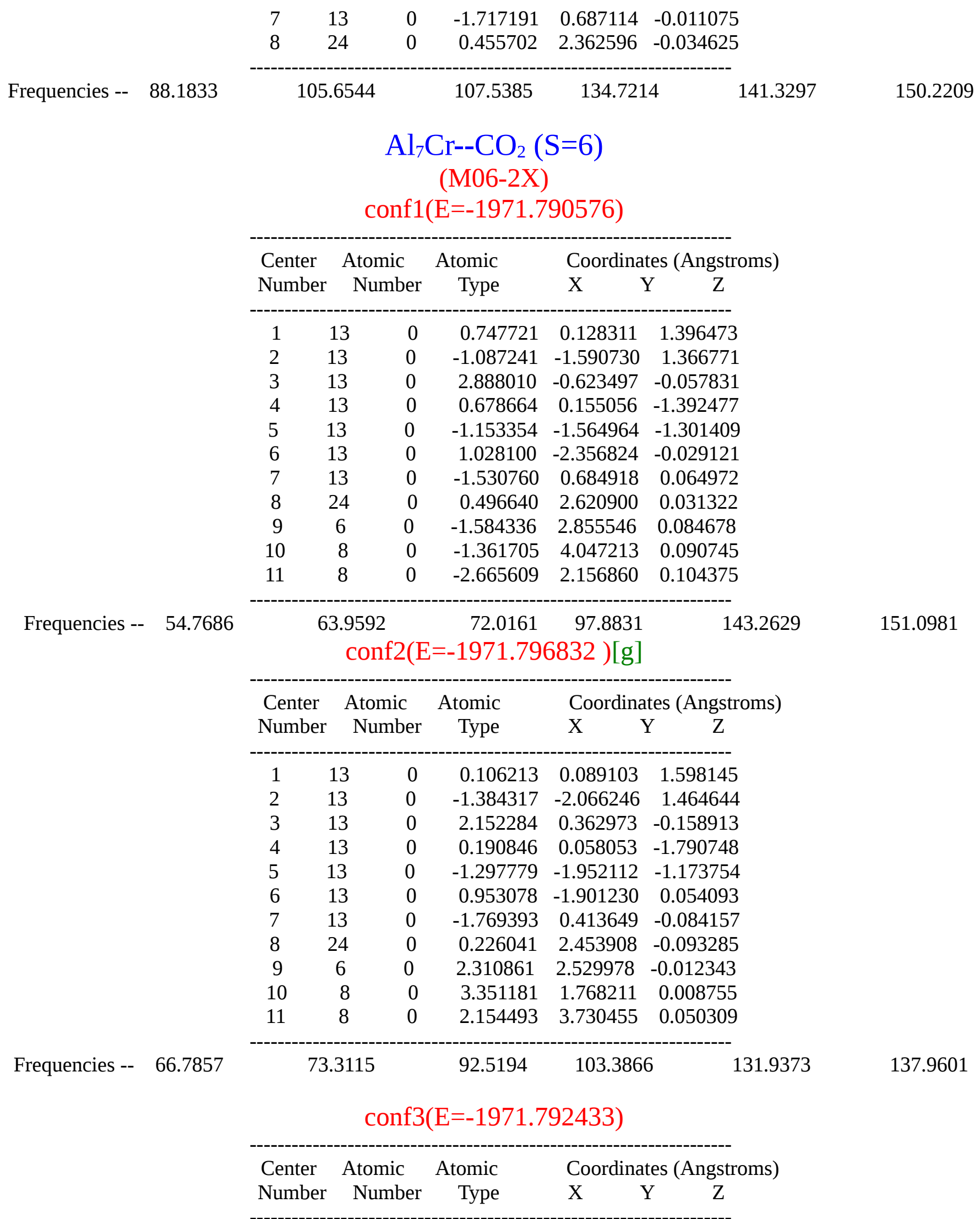




$\begin{array}{rrrrrr}1 & 13 & 0 & -0.404260 & -0.478239 & 2.519202 \\ 2 & 13 & 0 & -0.653432 & -2.751460 & 1.378021 \\ 3 & 13 & 0 & 1.482470 & 0.271102 & 0.905804 \\ 4 & 13 & 0 & 0.376329 & 0.305251 & -1.687209 \\ 5 & 13 & 0 & -1.465083 & -1.384676 & -0.753688 \\ 6 & 13 & 0 & 1.166954 & -1.862699 & -0.342649 \\ 7 & 13 & 0 & -1.377822 & 0.783245 & 0.475155 \\ 8 & 24 & 0 & 0.586471 & 2.771323 & -0.203381 \\ 9 & 6 & 0 & 0.809850 & 2.119459 & -2.373802 \\ 10 & 8 & 0 & 0.777542 & 1.159846 & -3.271258 \\ 11 & 8 & 0 & 1.044659 & 3.309336 & -2.514260\end{array}$

Frequencies -- 27.7616 (1)

$$
\begin{array}{crrr}
46.6018 & 68.4580 & 70.7903 & 103.4960 \\
\text { conf4(E=-1971.786803) } &
\end{array}
$$

Center Atomic Atomic Coordinates (Angstroms)

Number Number Type $\quad X \quad$ Y Z

$\begin{array}{rrrrrr}1 & 13 & 0 & 0.375261 & 0.304725 & 1.722326 \\ 2 & 13 & 0 & -1.393244 & -1.732854 & 1.389853 \\ 3 & 13 & 0 & 2.030456 & 0.122823 & -0.501792 \\ 4 & 13 & 0 & 0.085663 & 0.177998 & -2.165329 \\ 5 & 13 & 0 & -1.013958 & -2.096625 & -1.529575 \\ 6 & 13 & 0 & 0.951599 & -2.013318 & 0.155892 \\ 7 & 13 & 0 & -1.440713 & 0.341919 & -0.134357 \\ 8 & 24 & 0 & 0.393662 & 2.441150 & -0.171499 \\ 9 & 6 & 0 & 0.824227 & 2.410176 & 1.904868 \\ 10 & 8 & 0 & 0.871794 & 1.632451 & 2.931888 \\ 11 & 8 & 0 & 1.060484 & 3.591449 & 1.753047 \\ - & & & & & \end{array}$

Frequencies -- 50.5195

$\begin{array}{ccc}65.1526 & \begin{array}{c}80.1645 \\ (\mathrm{PBE}-\mathrm{PBE})\end{array} & 93.6502 \\ \text { conf1 }(\mathrm{E}=-1970.939620)\end{array}$

\begin{tabular}{|c|c|c|c|c|c|}
\hline \multirow{2}{*}{$\begin{array}{l}\text { Center } \\
\text { Number }\end{array}$} & \multirow{2}{*}{\multicolumn{2}{|c|}{$\begin{array}{l}\text { Atomic } \\
\text { Number }\end{array}$}} & \multirow{2}{*}{$\begin{array}{r}\text { Atomic } \\
\text { Type }\end{array}$} & \multicolumn{2}{|c|}{ Coordinates (Angstroms) } \\
\hline & & & & $\mathrm{X} \quad \mathrm{Y}$ & $\mathrm{Y} \quad \mathrm{Z}$ \\
\hline 1 & 13 & 0 & 0.549159 & 0.064916 & 1.485332 \\
\hline 2 & 13 & 0 & -1.535865 & -1.693865 & 0.409890 \\
\hline 3 & 13 & 0 & 2.054024 & -0.065165 & -0.647566 \\
\hline 4 & 13 & 0 & -0.376036 & 0.055470 & -1.609510 \\
\hline 5 & 13 & 0 & 0.421340 & -2.322462 & -1.461727 \\
\hline 6 & 13 & 0 & 1.107060 & -2.325847 & 0.952796 \\
\hline 7 & 13 & 0 & -1.736029 & 0.926892 & 0.479001 \\
\hline 8 & 24 & 0 & 0.608445 & 2.179872 & -0.221346 \\
\hline 9 & 6 & 0 & -1.206284 & 3.010433 & 0.311571 \\
\hline 10 & 8 & 0 & -2.427537 & 2.656238 & 0.679463 \\
\hline 11 & 8 & 0 & -0.716401 & 4.135060 & 0.158344 \\
\hline & 1.22 & & 110.1718 & 111.782 & 130.6513 \\
\hline
\end{tabular}

134.5695

Frequencies -- 26.5270 


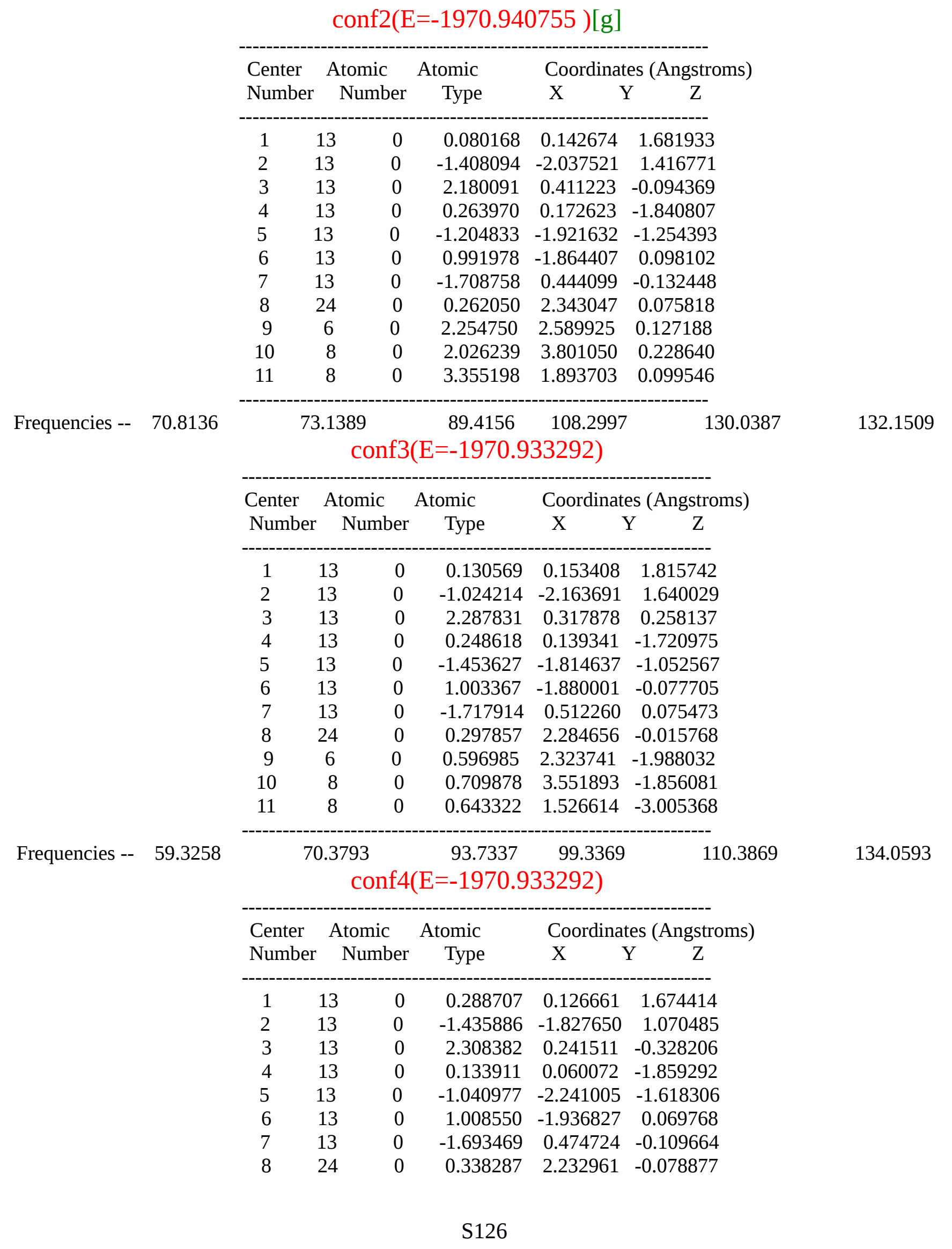




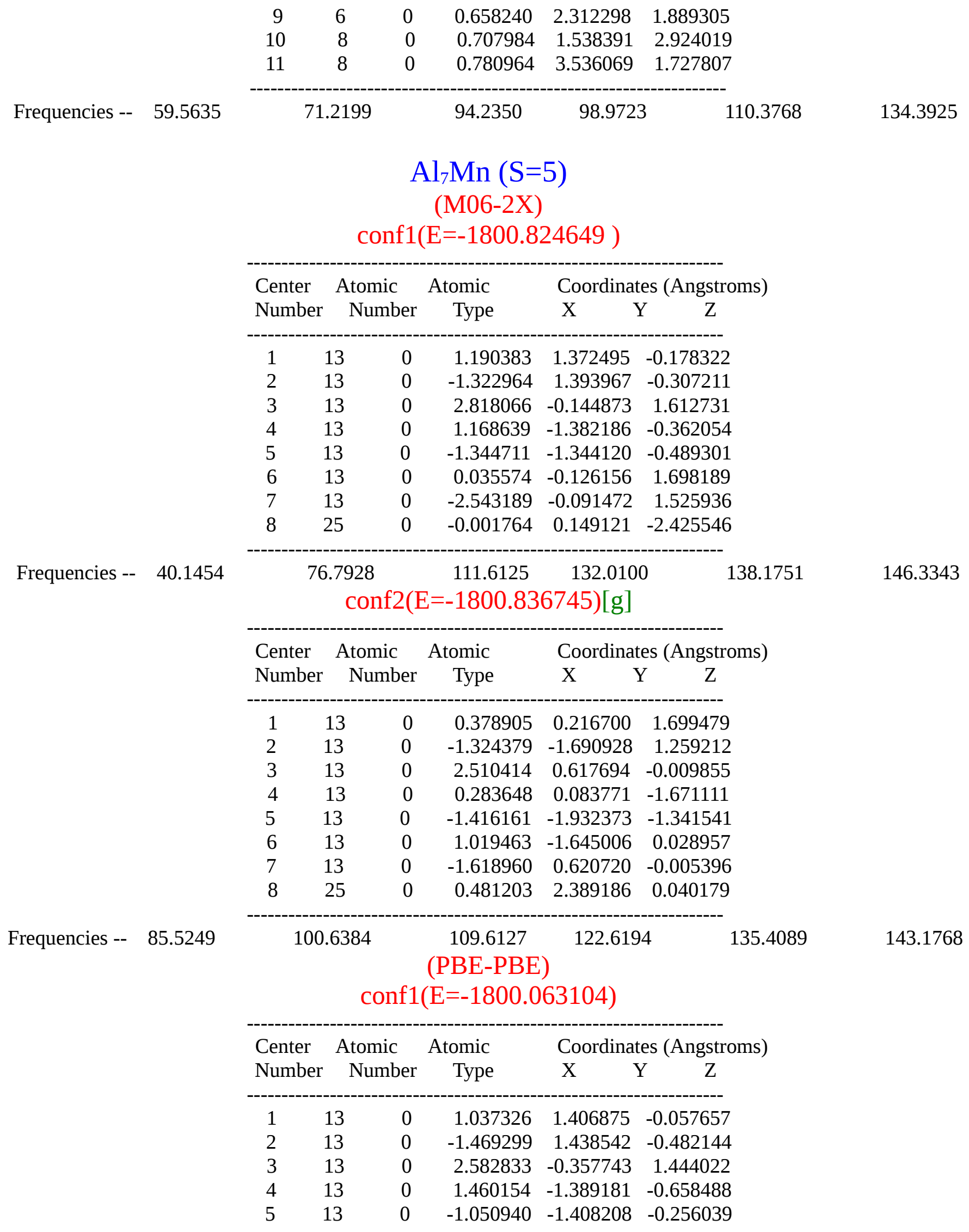




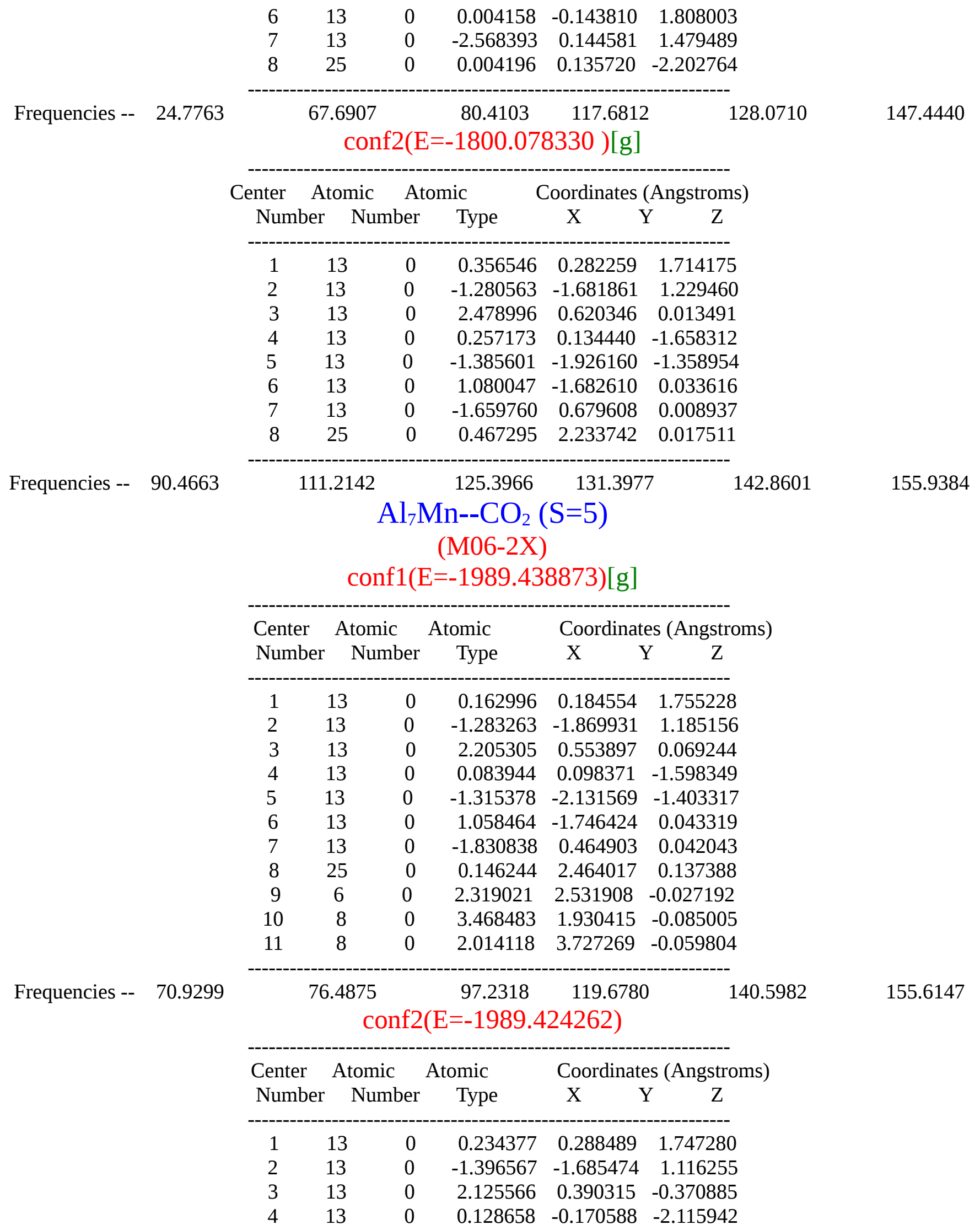




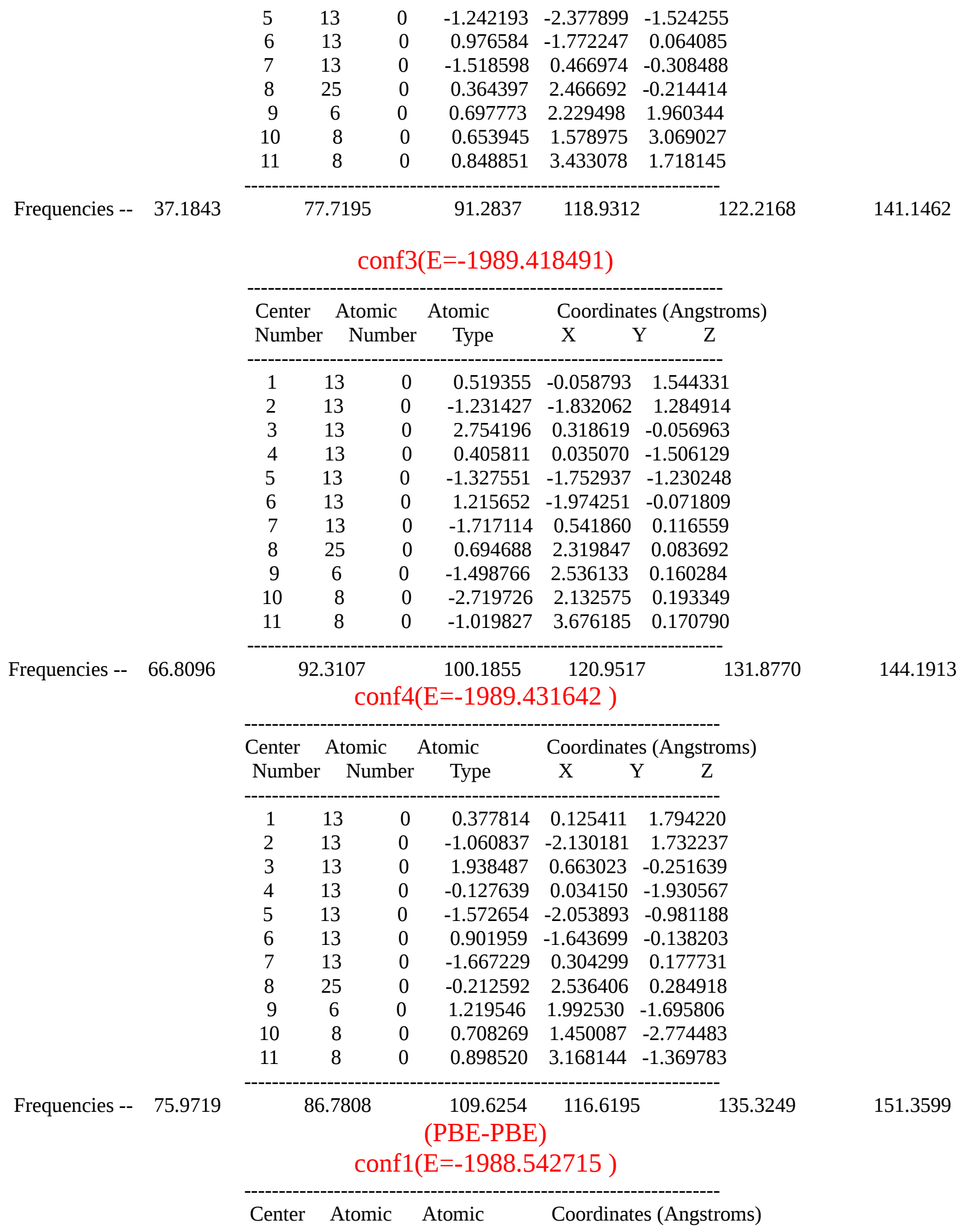




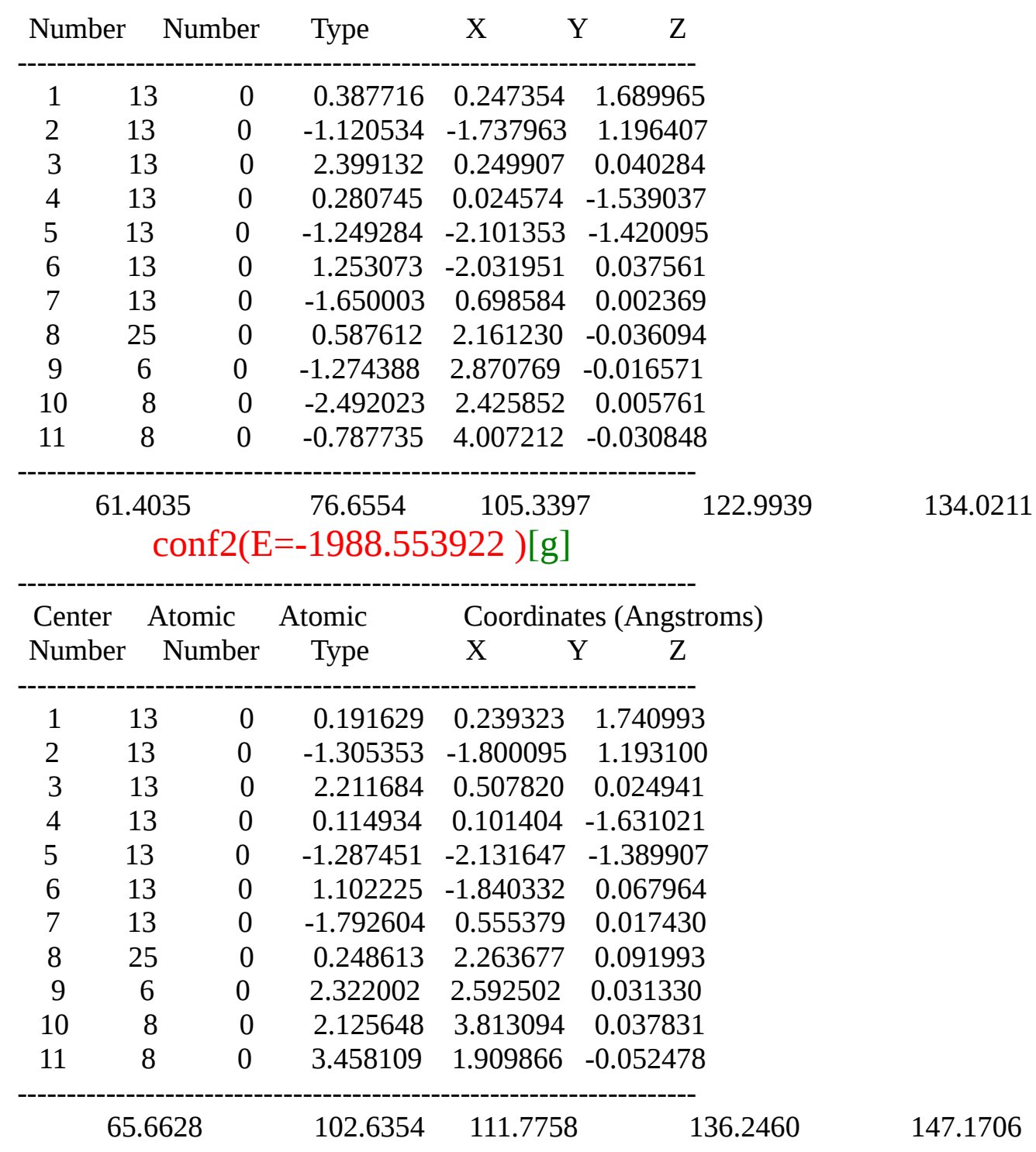

Frequencies -- 62.9860

Frequencies -- 29.1230

$$
\text { conf3(E=-1988.547870) }
$$

\begin{tabular}{|c|c|c|c|c|c|}
\hline \multirow{2}{*}{$\begin{array}{l}\text { Center } \\
\text { Number }\end{array}$} & \multirow{2}{*}{\multicolumn{2}{|c|}{$\begin{array}{l}\text { Atomic } \\
\text { Number }\end{array}$}} & \multirow{2}{*}{$\begin{array}{r}\text { Atomic } \\
\text { Type }\end{array}$} & \multicolumn{2}{|c|}{ Coordinates (Angstro } \\
\hline & & & & $X \quad Y$ & $\begin{array}{ll}Y & Z\end{array}$ \\
\hline 1 & 13 & 0 & 0.373542 & 0.113980 & 1.701743 \\
\hline 2 & 13 & 0 & 3 & 43462 & 783 \\
\hline 3 & 13 & 0 & & 0.361023 & 0225 \\
\hline 4 & 13 & 0 & & -0.010315 & 7551 \\
\hline 5 & 13 & 0 & -1.295 & -2.163642 & -1.473050 \\
\hline 6 & 13 & 0 & & -1.836264 & -0.013068 \\
\hline 7 & 13 & 0 & -1.639 & 0.614242 & -0.023090 \\
\hline 8 & 25 & 0 & 0.4526 & 2.098528 & -0.178195 \\
\hline 9 & 6 & 0 & 0.868097 & 2.341816 & 1.722100 \\
\hline 10 & 8 & 0 & 0.962259 & 3.554324 & 1.504648 \\
\hline 11 & 8 & 0 & 0.947635 & 1.608265 & 2.780549 \\
\hline
\end{tabular}




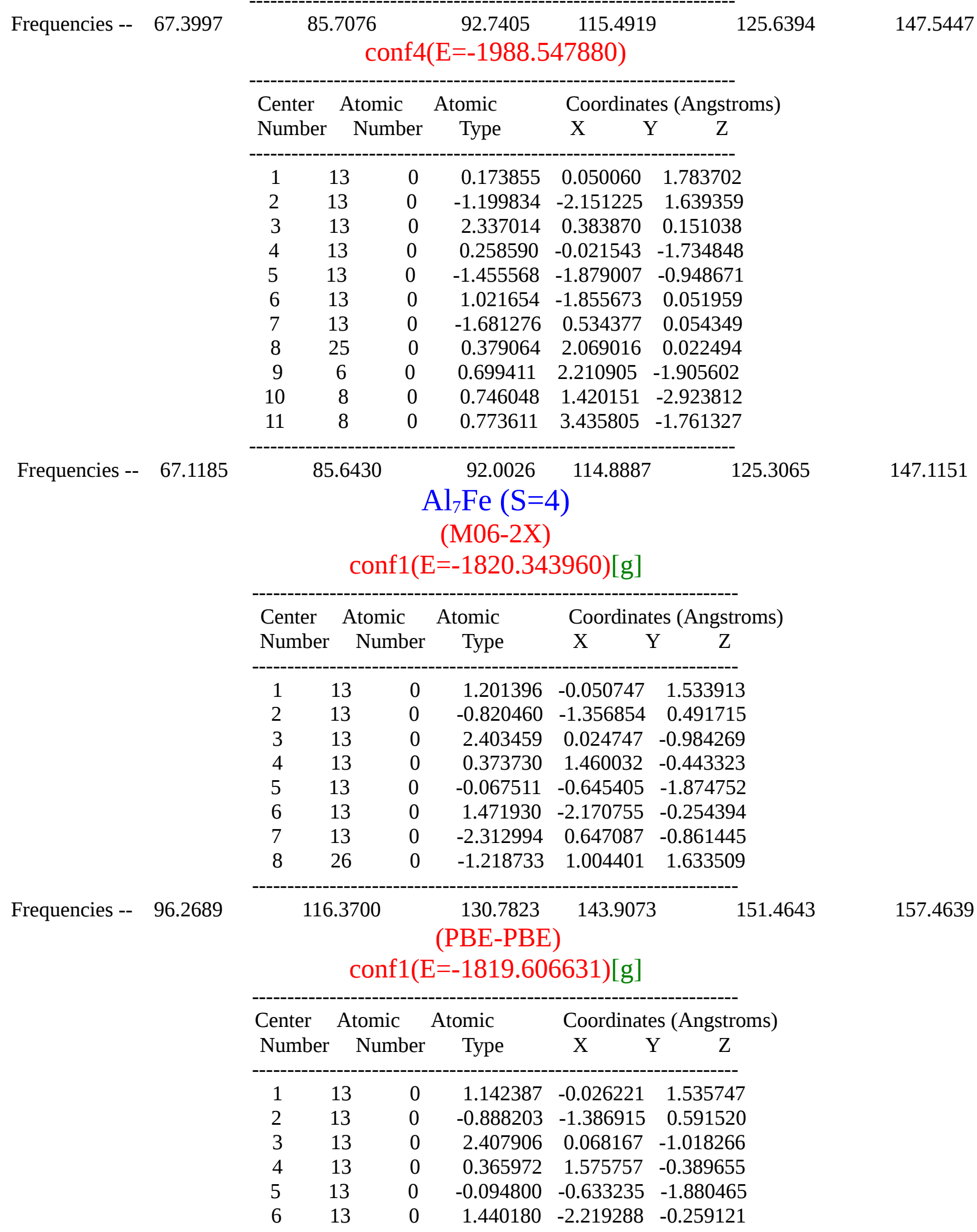




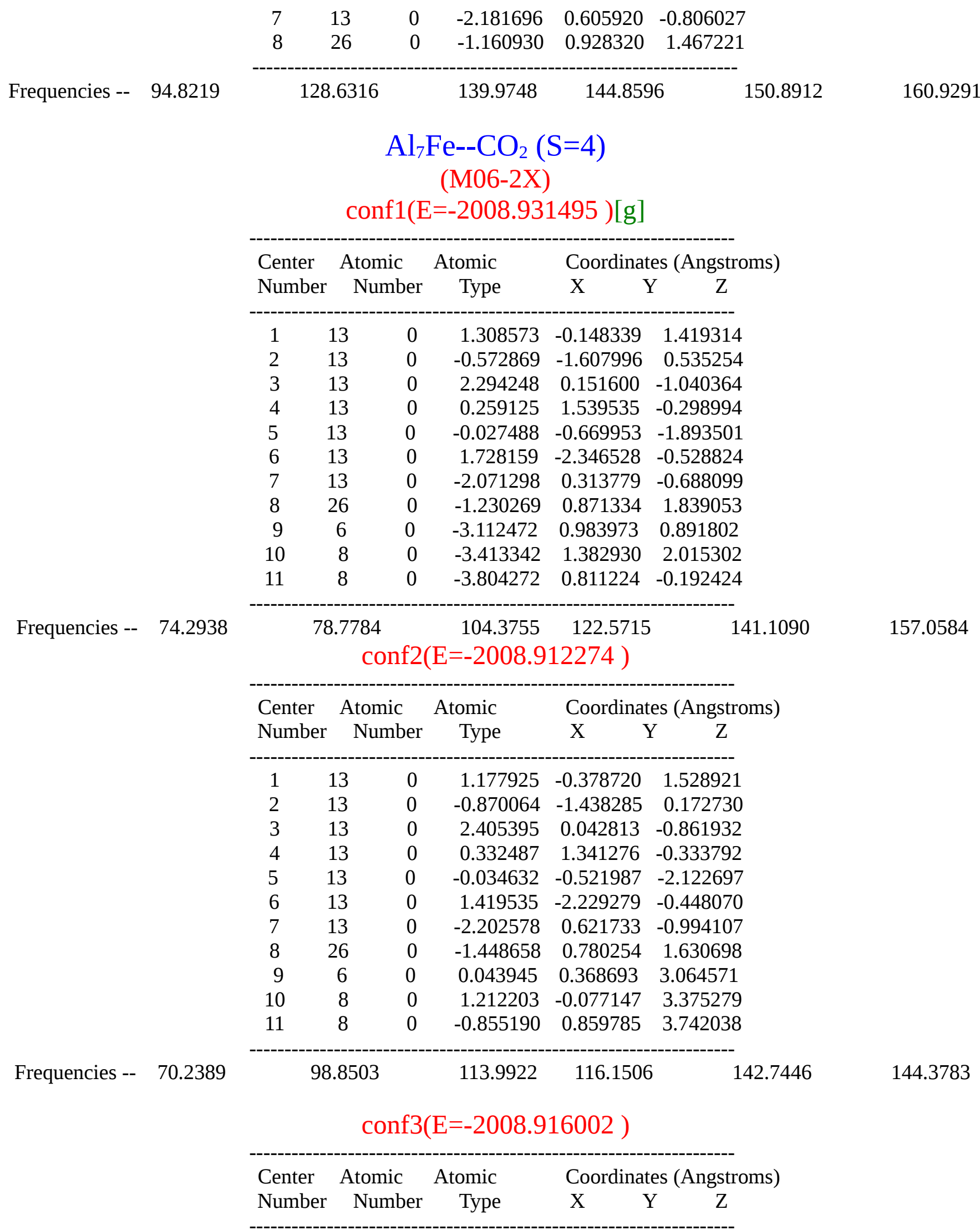




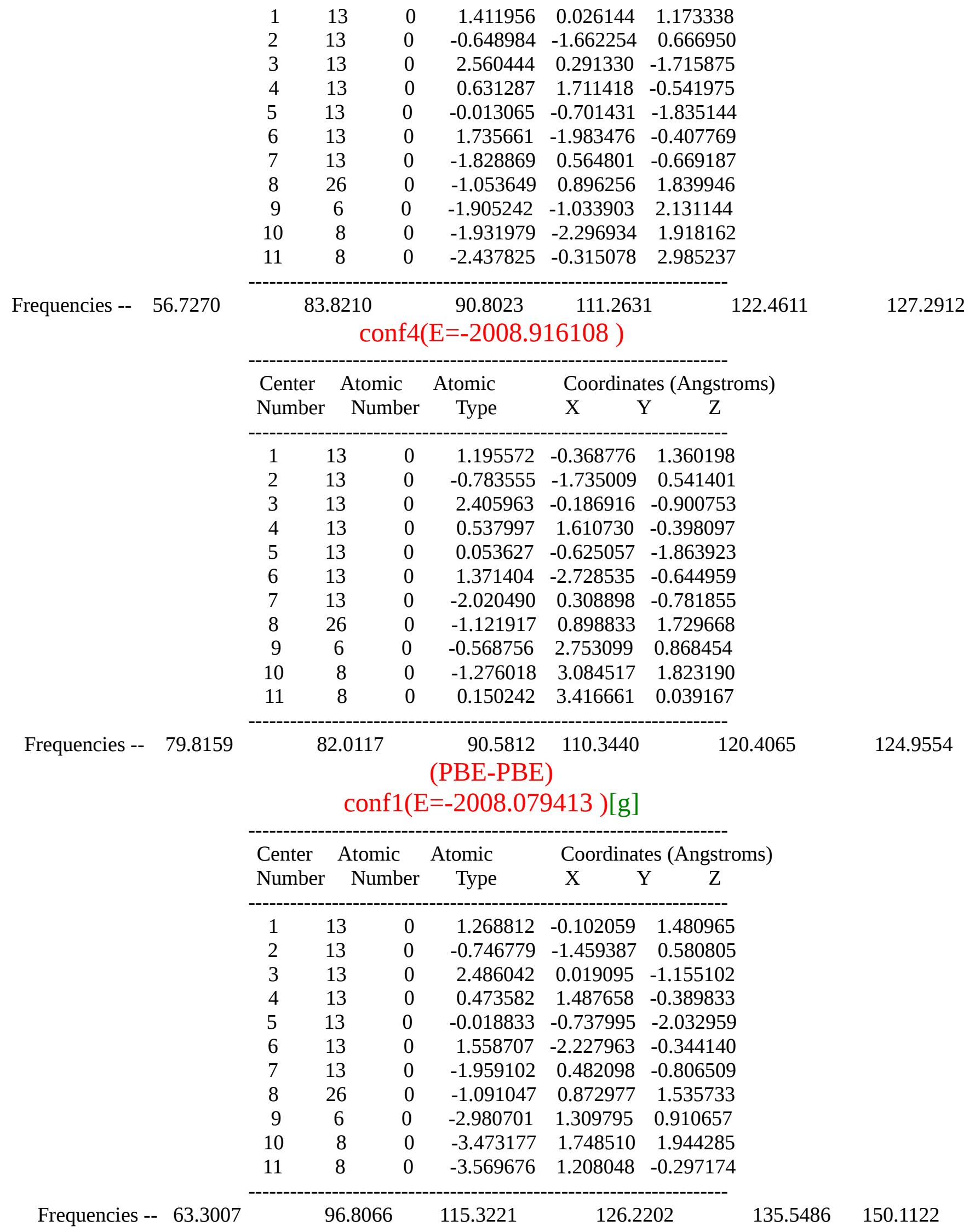




\section{conf2(E=-2008.073013 )}

\begin{tabular}{|c|c|c|c|c|c|}
\hline \multirow{2}{*}{$\begin{array}{l}\text { Center } \\
\text { Number }\end{array}$} & \multirow{2}{*}{\multicolumn{2}{|c|}{$\begin{array}{l}\text { Atomic } \\
\text { Number }\end{array}$}} & \multirow{2}{*}{$\begin{array}{r}\text { Atomic } \\
\text { Type }\end{array}$} & \multicolumn{2}{|c|}{ Coordinates (Angstror } \\
\hline & & & & $\mathrm{X} \quad \mathrm{Y}$ & $\mathrm{Y} \quad \mathrm{Z}$ \\
\hline 1 & 13 & 0 & 1.176159 & -0.254440 & 1.446126 \\
\hline 2 & 13 & 0 & -0.899128 & -1.447760 & 0.344806 \\
\hline 3 & 13 & 0 & 2.384350 & 0.104060 & -1.072198 \\
\hline 4 & 13 & 0 & 0.320876 & 1.466402 & -0.356000 \\
\hline 5 & 13 & 0 & -0.021499 & -0.626572 & -2.224096 \\
\hline 6 & 13 & 0 & 1.383552 & -2.273239 & -0.513069 \\
\hline 7 & 13 & 0 & -2.042356 & 0.481172 & -1.098371 \\
\hline 8 & 26 & 0 & -1.131897 & 0.718090 & 1.476681 \\
\hline 9 & 6 & 0 & -0.070932 & 0.651287 & 3.044785 \\
\hline 10 & 8 & 0 & 1.135363 & 0.201003 & 3.281492 \\
\hline 11 & 8 & 0 & -0.924644 & 1.179841 & 3.754838 \\
\hline
\end{tabular}

Frequencies -- 55.4922

$\begin{array}{rrr}65.7611 & 80.9522 & 106.6646\end{array}$
132.3464

136.9944 conf3(E=-2008.074621)

\begin{tabular}{|c|c|c|c|c|c|}
\hline \multirow{2}{*}{$\begin{array}{l}\text { Center } \\
\text { Number }\end{array}$} & \multicolumn{2}{|c|}{ Atomic } & \multirow{2}{*}{$\begin{array}{l}\text { Atomic } \\
\text { Type }\end{array}$} & \multicolumn{2}{|c|}{ Coordinates (Angstror } \\
\hline & & Number & & $\mathrm{X}$ & $\mathrm{Y} \quad \mathrm{Z}$ \\
\hline 1 & 13 & 0 & 1.384603 & -0.007267 & 1.285510 \\
\hline 2 & 13 & 0 & -0.719363 & -1.485483 & 0.578509 \\
\hline 3 & 13 & 0 & 2.555658 & 0.160301 & -1.394246 \\
\hline 4 & 13 & 0 & 0.541222 & 1.582247 & -0.604354 \\
\hline 5 & 13 & 0 & -0.037736 & -0.699877 & -1.908428 \\
\hline 6 & 13 & 0 & 1.677446 & -2.073489 & -0.366430 \\
\hline 7 & 13 & 0 & -1.964488 & 0.630412 & -0.770505 \\
\hline 8 & 26 & 0 & -0.867914 & 0.943190 & 1.500629 \\
\hline 9 & 6 & 0 & -1.918040 & -0.463450 & 2.200262 \\
\hline 10 & 8 & 0 & -2.036387 & -1.728848 & 1.943065 \\
\hline 11 & 8 & 0 & -2.473527 & 0.308721 & 2.986402 \\
\hline
\end{tabular}

Frequencies -- 70.5059

83.3958 $107.6101 \quad 125.9506$ 138.6201

147.1624 conf4(E=-2008.074663)

\begin{tabular}{|c|c|c|c|c|c|}
\hline \multirow{2}{*}{$\begin{array}{l}\text { Center } \\
\text { Number }\end{array}$} & \multicolumn{2}{|c|}{ Atomic } & \multirow{2}{*}{$\begin{array}{l}\text { Atomic } \\
\text { Type }\end{array}$} & \multicolumn{2}{|c|}{ Coordinates (Angstro } \\
\hline & & Number & & $\mathrm{X}$ & $Y \quad Z$ \\
\hline 1 & 13 & 0 & 1.256712 & -0.296239 & 1.393688 \\
\hline 2 & 13 & 0 & -0.796889 & -1.611901 & 0.464750 \\
\hline 3 & 13 & 0 & 2.479 & -0.221933 & -0.968596 \\
\hline 4 & 13 & 0 & 0.5606 & 1.513795 & -0.436774 \\
\hline 5 & 13 & 0 & 0.013817 & -0.672197 & -1.922432 \\
\hline 6 & 13 & 0 & & -2.558372 & -0.451143 \\
\hline 7 & 13 & 0 & -2.0145 & 0.455368 & -0.743329 \\
\hline 8 & 26 & 0 & -0.951844 & 0.761436 & 1.542774 \\
\hline
\end{tabular}




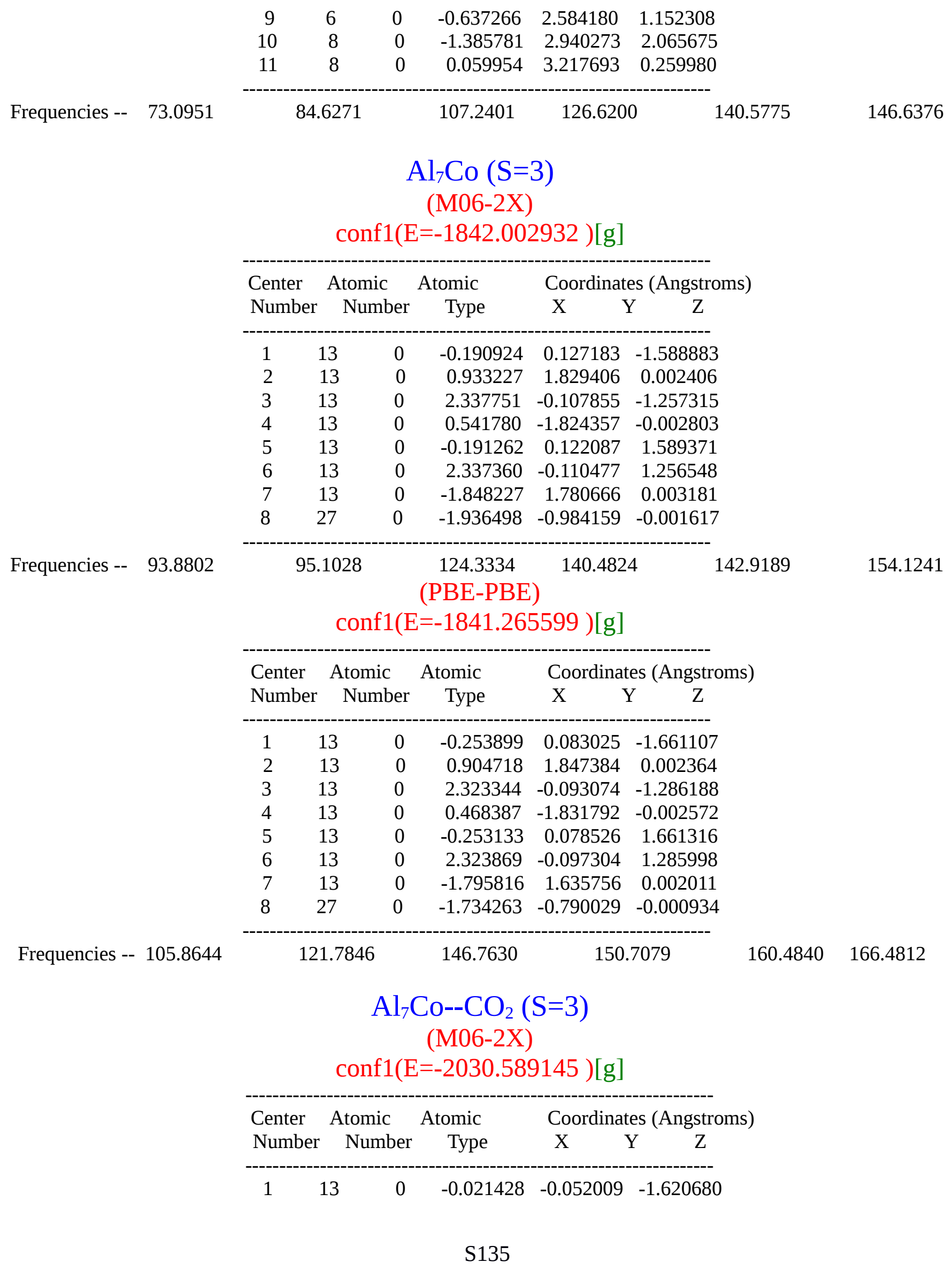




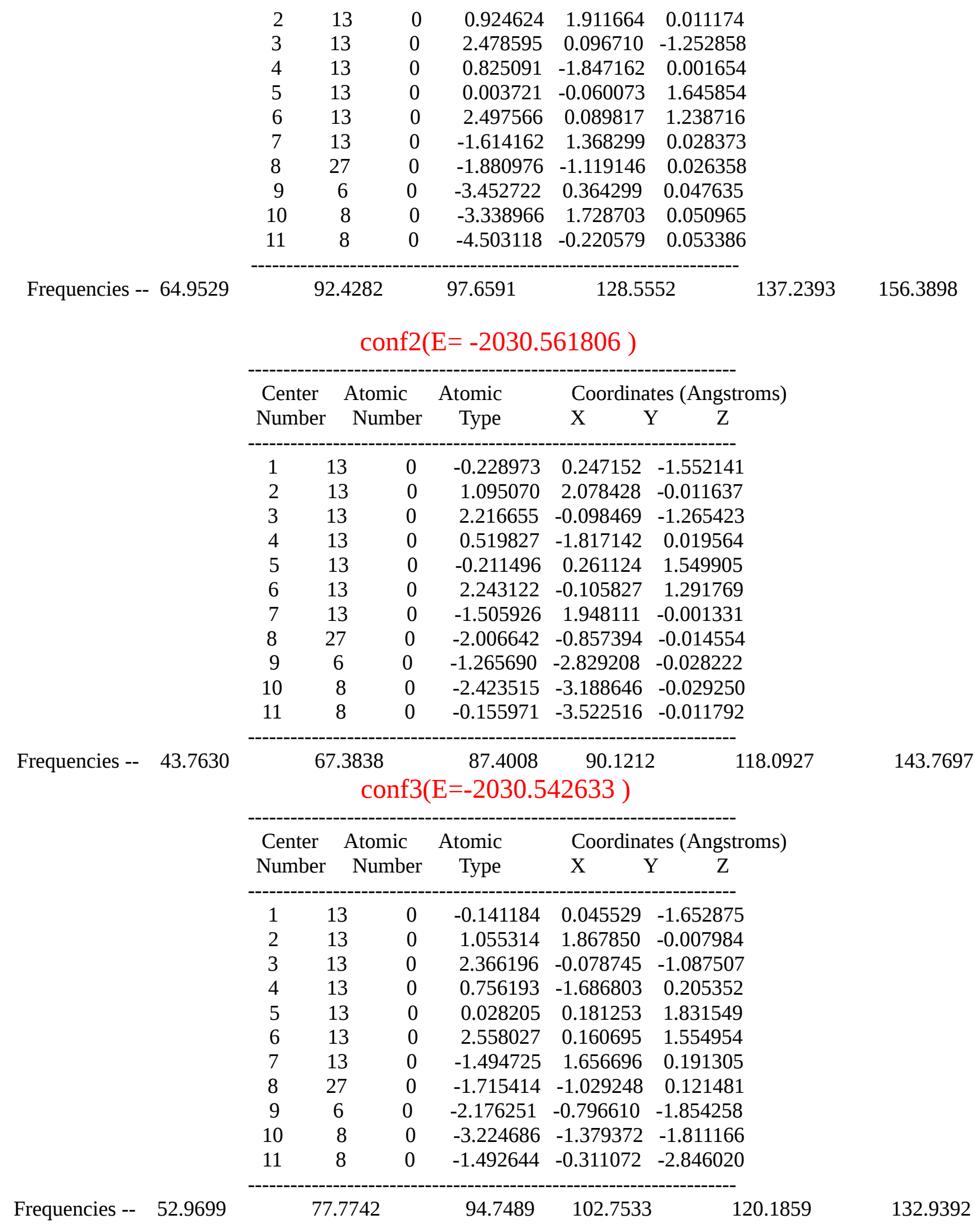




\section{$\operatorname{conf} 4(E=-2030.588290)$}

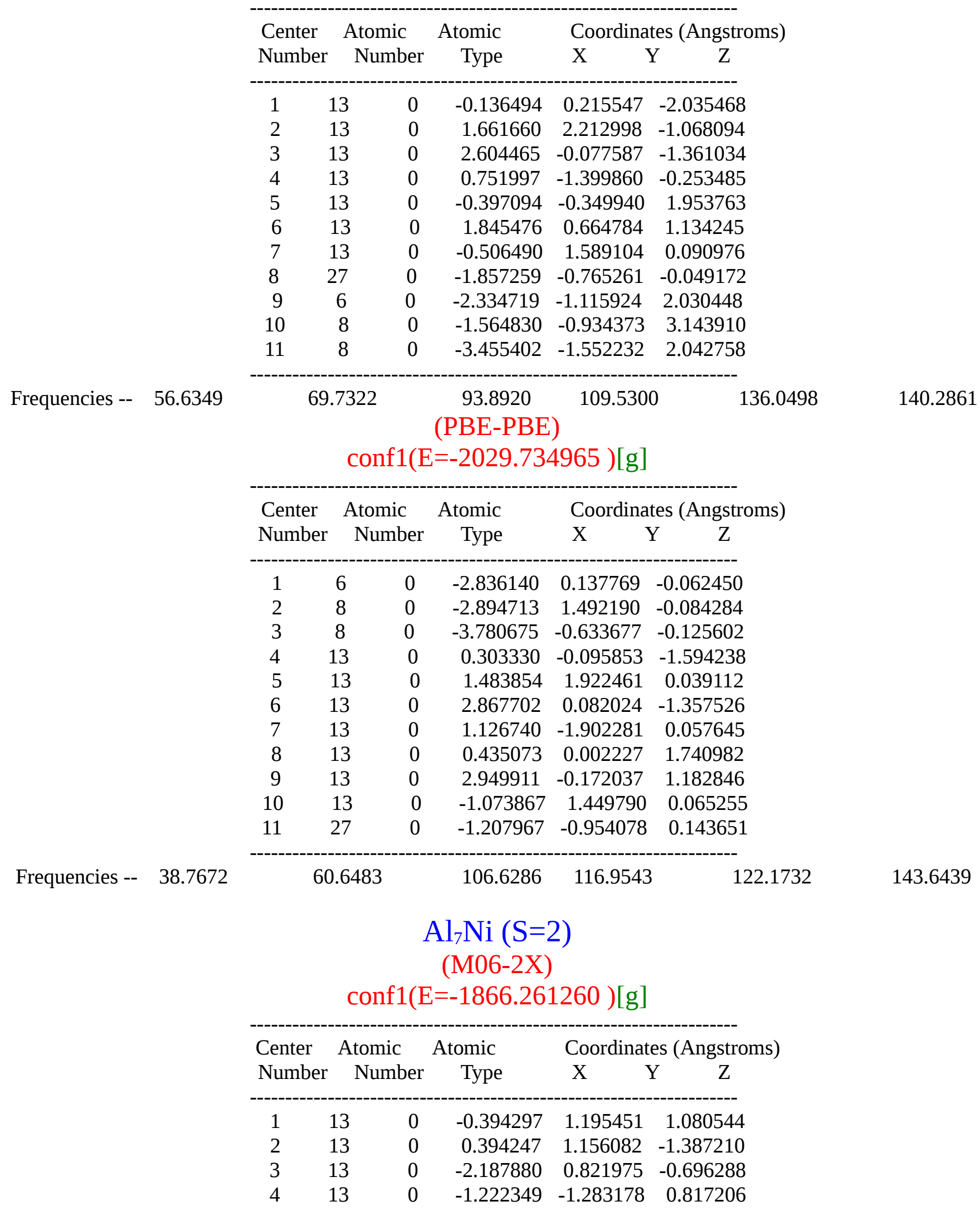




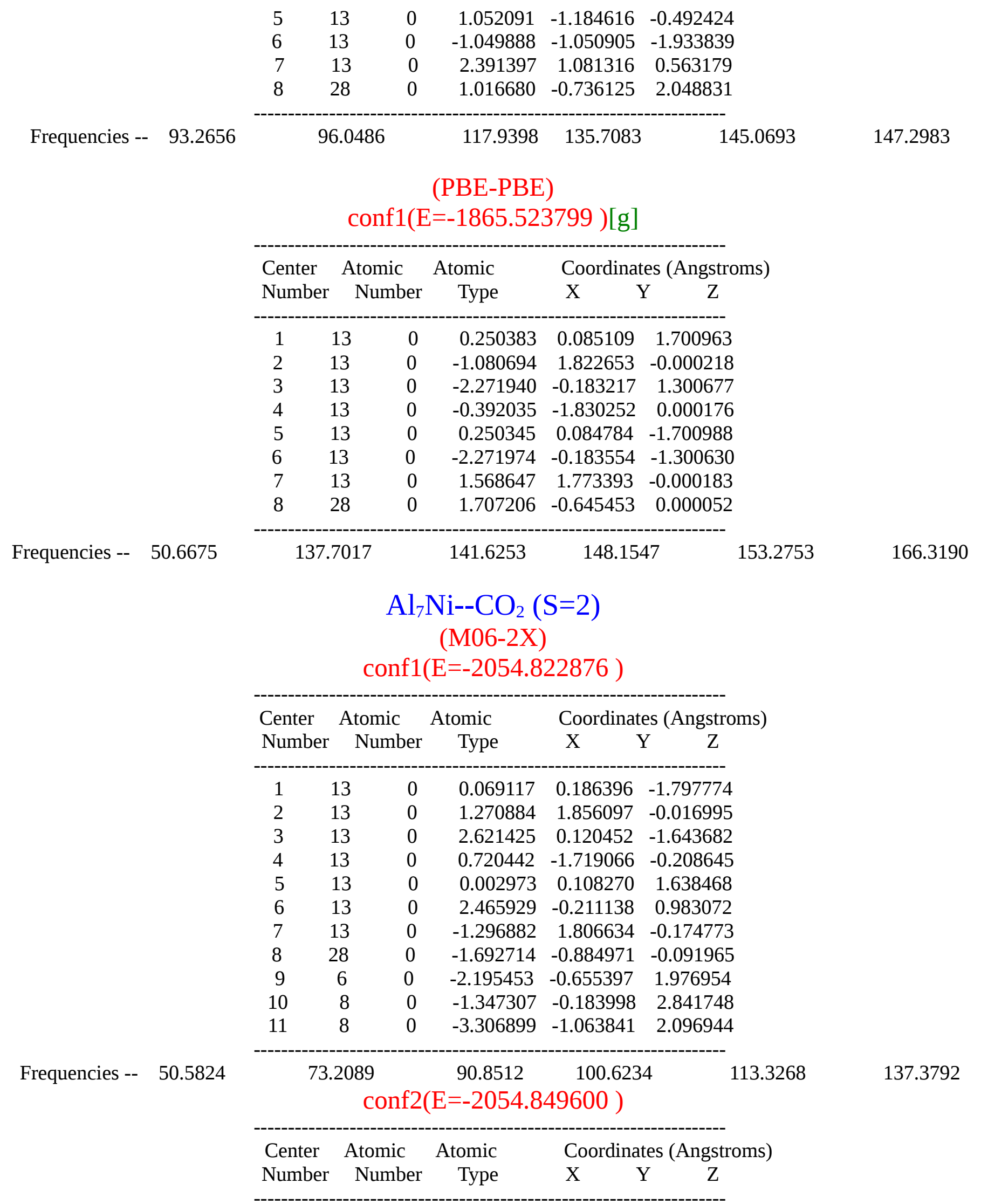




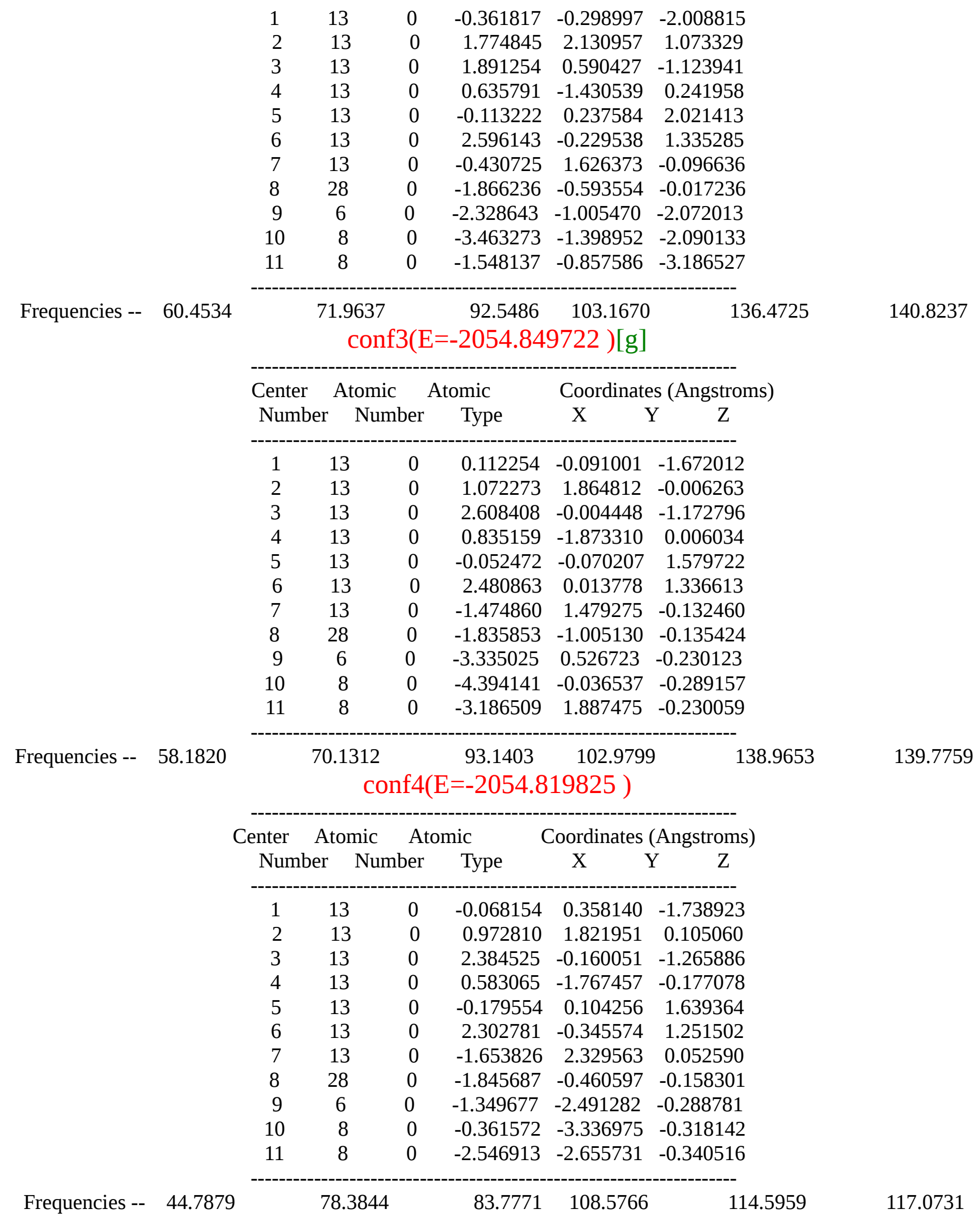




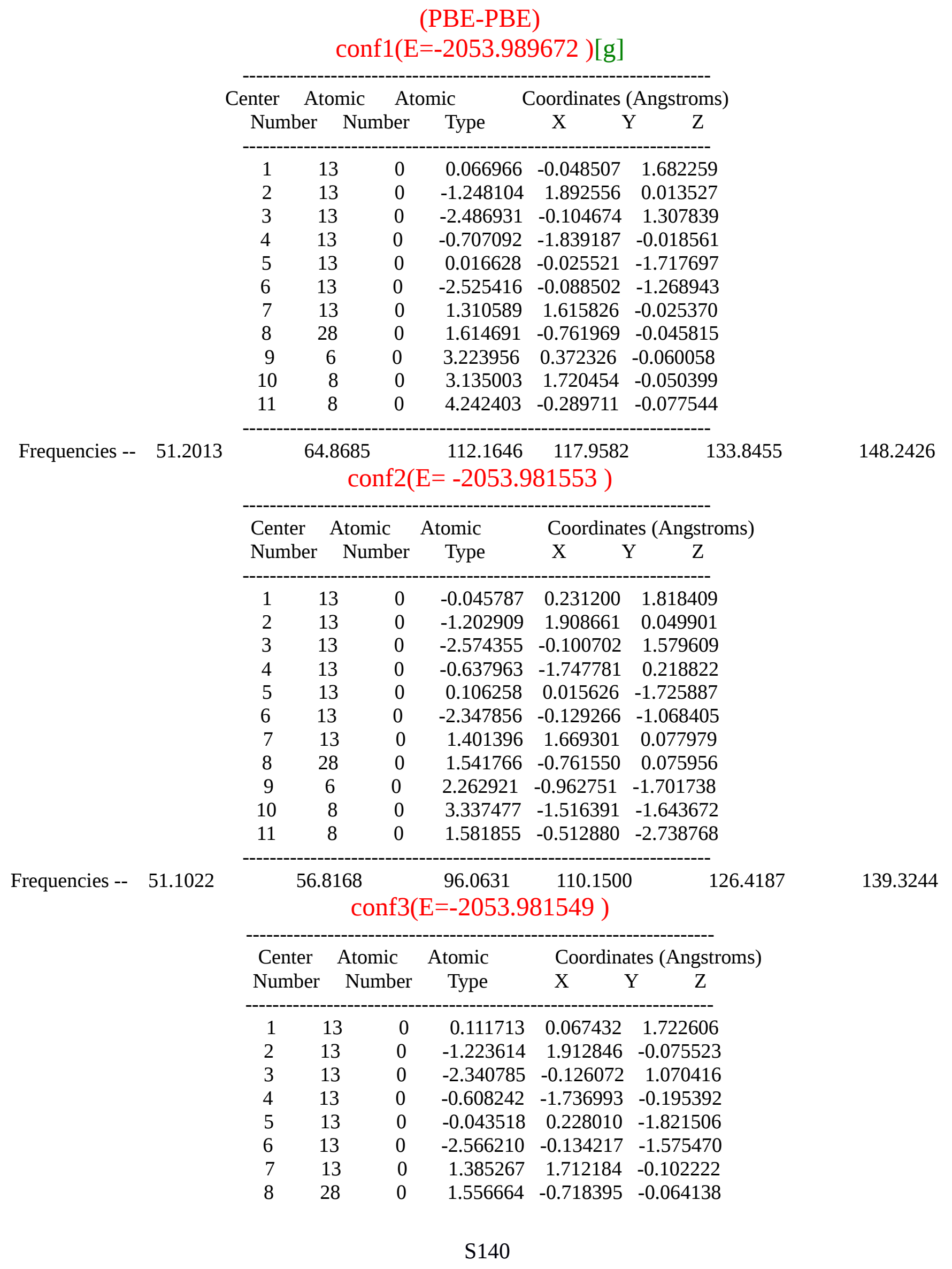




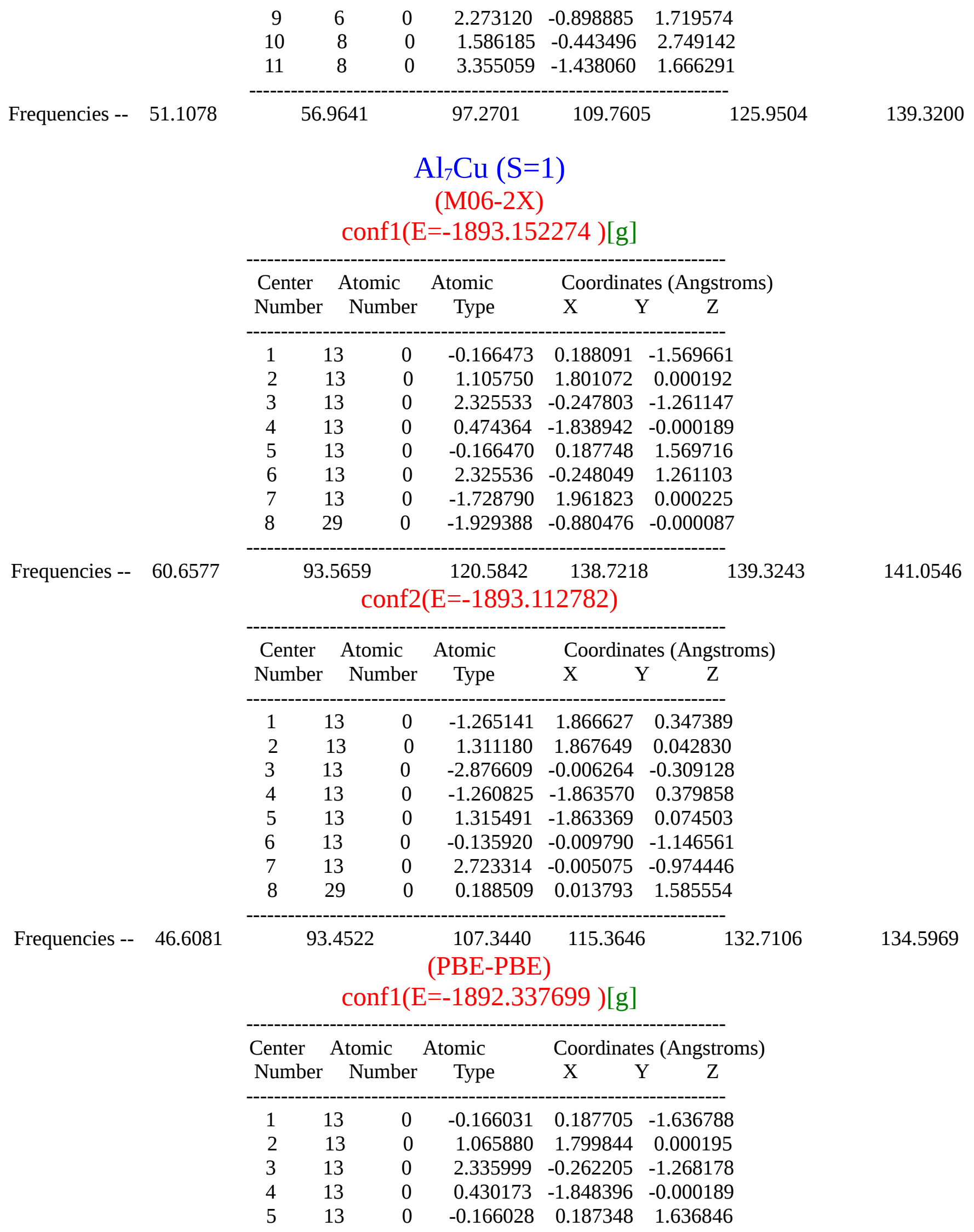




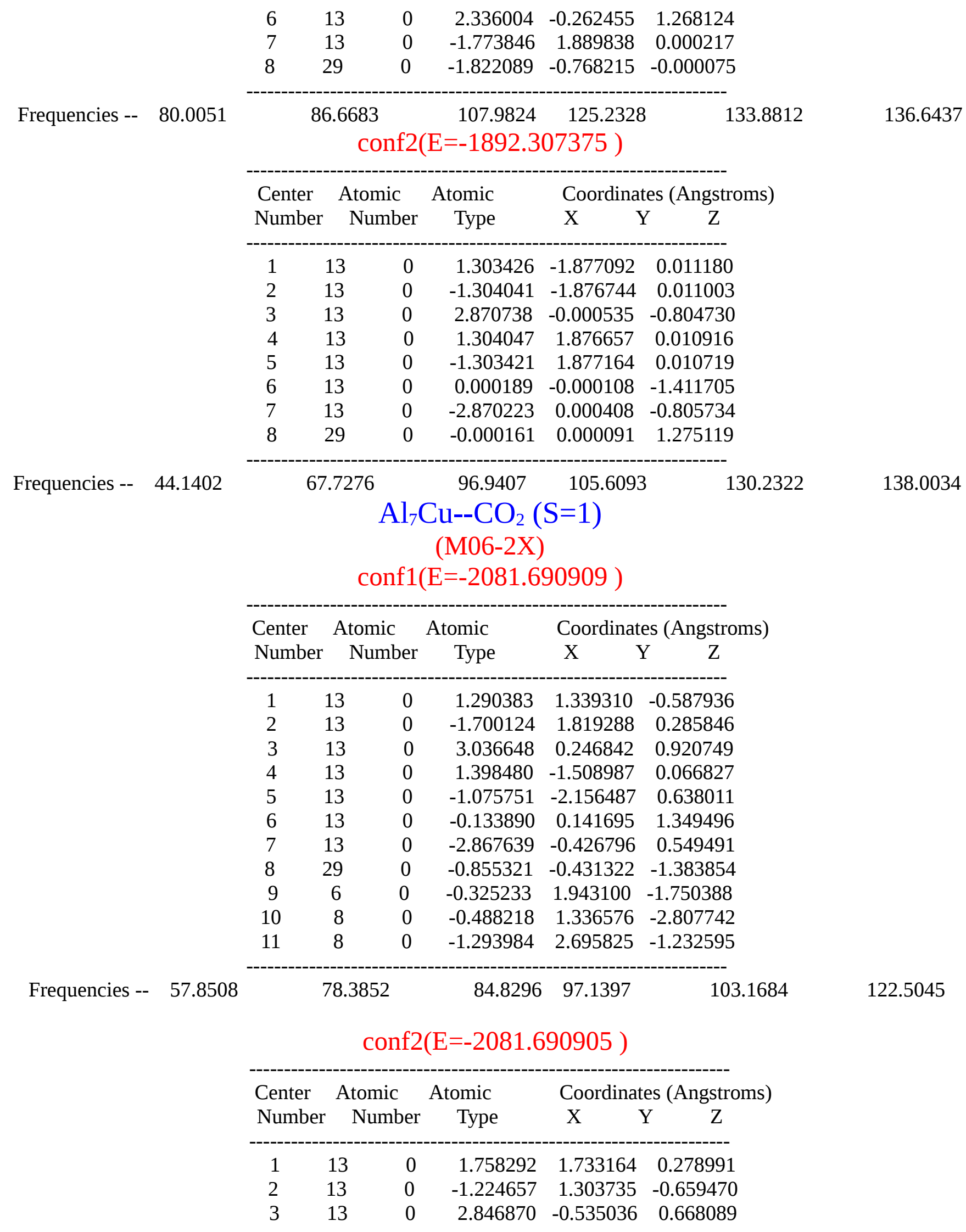




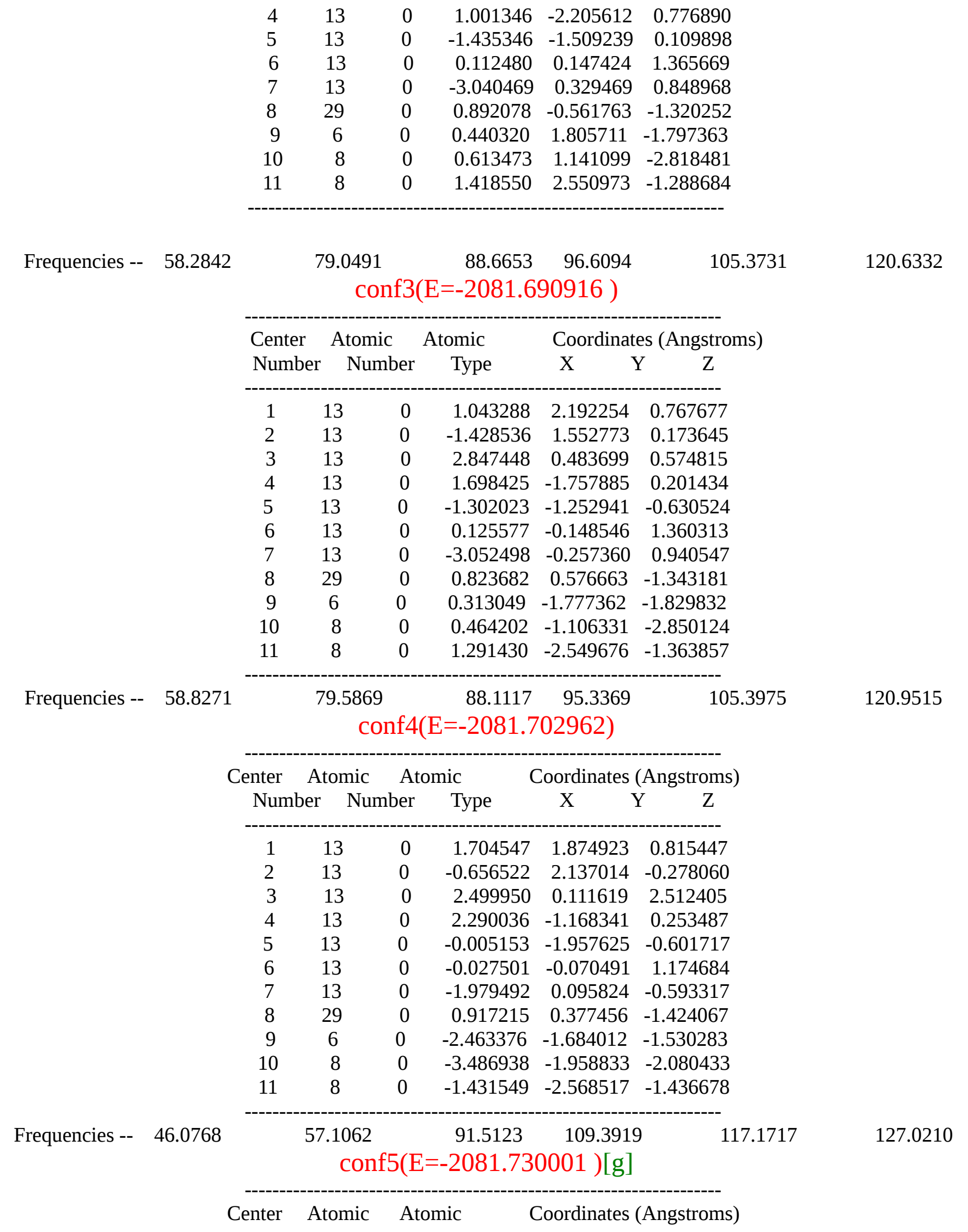




\begin{tabular}{|c|c|c|c|c|c|}
\hline Number & & Number & Type & $X$ & Z \\
\hline 1 & 13 & 0 & -0.039139 & -0.063029 & -1.622635 \\
\hline 2 & 13 & 0 & 0.977486 & 1.885207 & -0.001578 \\
\hline 3 & 13 & 0 & 2.466801 & 0.000265 & -1.261917 \\
\hline 4 & 13 & 0 & 0.749977 & -1.876903 & -0.001780 \\
\hline 5 & 13 & 0 & -0.013103 & -0.064457 & 1.632941 \\
\hline 6 & 13 & 0 & 2.487283 & -0.000853 & 1.233462 \\
\hline 7 & 13 & 0 & -1.583576 & 1.430245 & 0.018116 \\
\hline 8 & 29 & 0 & -1.884627 & -1.067447 & 0.019647 \\
\hline 9 & 6 & 0 & -3.497286 & 0.486784 & 0.030760 \\
\hline 10 & 8 & 0 & -3.282753 & 1.851099 & 0.030657 \\
\hline 11 & 8 & 0 & -4.590018 & 0.002129 & 0.037659 \\
\hline
\end{tabular}

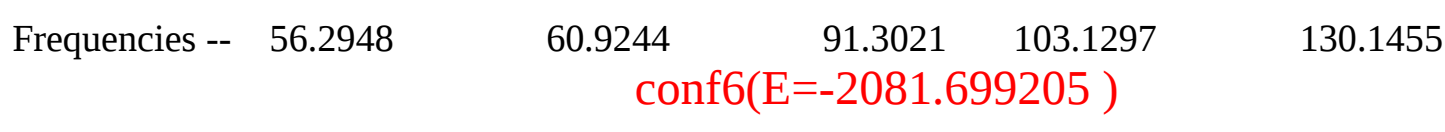

133.0503

\begin{tabular}{|c|c|c|c|c|c|}
\hline Center & Atom & & & Coordinates & (Angstroms) \\
\hline Number & & Number & Type & $\mathrm{X}$ & $\begin{array}{ll}Y & Z\end{array}$ \\
\hline 1 & 13 & 0 & -0.104129 & 0.328909 & -1.689694 \\
\hline 2 & 13 & 0 & 1.039087 & 1.853060 & 0.049372 \\
\hline 3 & 13 & 0 & 2.328705 & -0.242671 & -1.262800 \\
\hline 4 & 13 & 0 & 0.549983 & -1.802024 & -0.072245 \\
\hline 5 & 13 & 0 & -0.142968 & 0.221427 & 1.658705 \\
\hline 6 & 13 & 0 & 2.298937 & -0.330901 & 1.253387 \\
\hline 7 & 13 & 0 & -1.636687 & 2.401519 & 0.042128 \\
\hline 8 & 29 & 0 & -1.794622 & -0.610460 & -0.063577 \\
\hline 9 & 6 & 0 & -1.368813 & -2.755097 & -0.160105 \\
\hline 10 & 8 & 0 & -2.498342 & -3.138866 & -0.203467 \\
\hline 11 & 8 & 0 & -0.243971 & -3.427694 & -0.162302 \\
\hline
\end{tabular}

Frequencies -- 8.4785

\begin{tabular}{|c|c|c|c|c|c|}
\hline \multicolumn{6}{|c|}{$\begin{array}{r}61.4868 \\
\text { Con }\end{array}$} \\
\hline \multirow{2}{*}{\multicolumn{2}{|c|}{$\begin{array}{l}\text { Center } \\
\text { Number }\end{array}$}} & & \multirow{2}{*}{$\begin{array}{l}\text { Atomic } \\
\text { Type }\end{array}$} & \multicolumn{2}{|c|}{ Coordinates (Angstroms) } \\
\hline & & & & $\mathrm{X}$ & $\mathrm{Y} \quad \mathrm{Z}$ \\
\hline 1 & 13 & 0 & -0.492579 & -0.047005 & -2.026417 \\
\hline 2 & 13 & 0 & 1.870119 & 1.990612 & 1.189853 \\
\hline 3 & 13 & 0 & 1.861305 & 0.644475 & -1.164483 \\
\hline 4 & 13 & 0 & 0.546285 & -1.403016 & 0.072137 \\
\hline 5 & 13 & 0 & -0.125059 & 0.139826 & 2.001055 \\
\hline 6 & 13 & 0 & 2.559451 & -0.407176 & 1.240950 \\
\hline 7 & 13 & 0 & -0.354364 & 1.725577 & 0.009569 \\
\hline 8 & 29 & 0 & -1.932997 & -0.423530 & 0.000467 \\
\hline 9 & 6 & 0 & -2.545394 & -0.603276 & -2.147624 \\
\hline 10 & 8 & 0 & -3.697275 & -0.902777 & -2.261601 \\
\hline 11 & 8 & 0 & -1.713603 & -0.397110 & -3.230717 \\
\hline
\end{tabular}




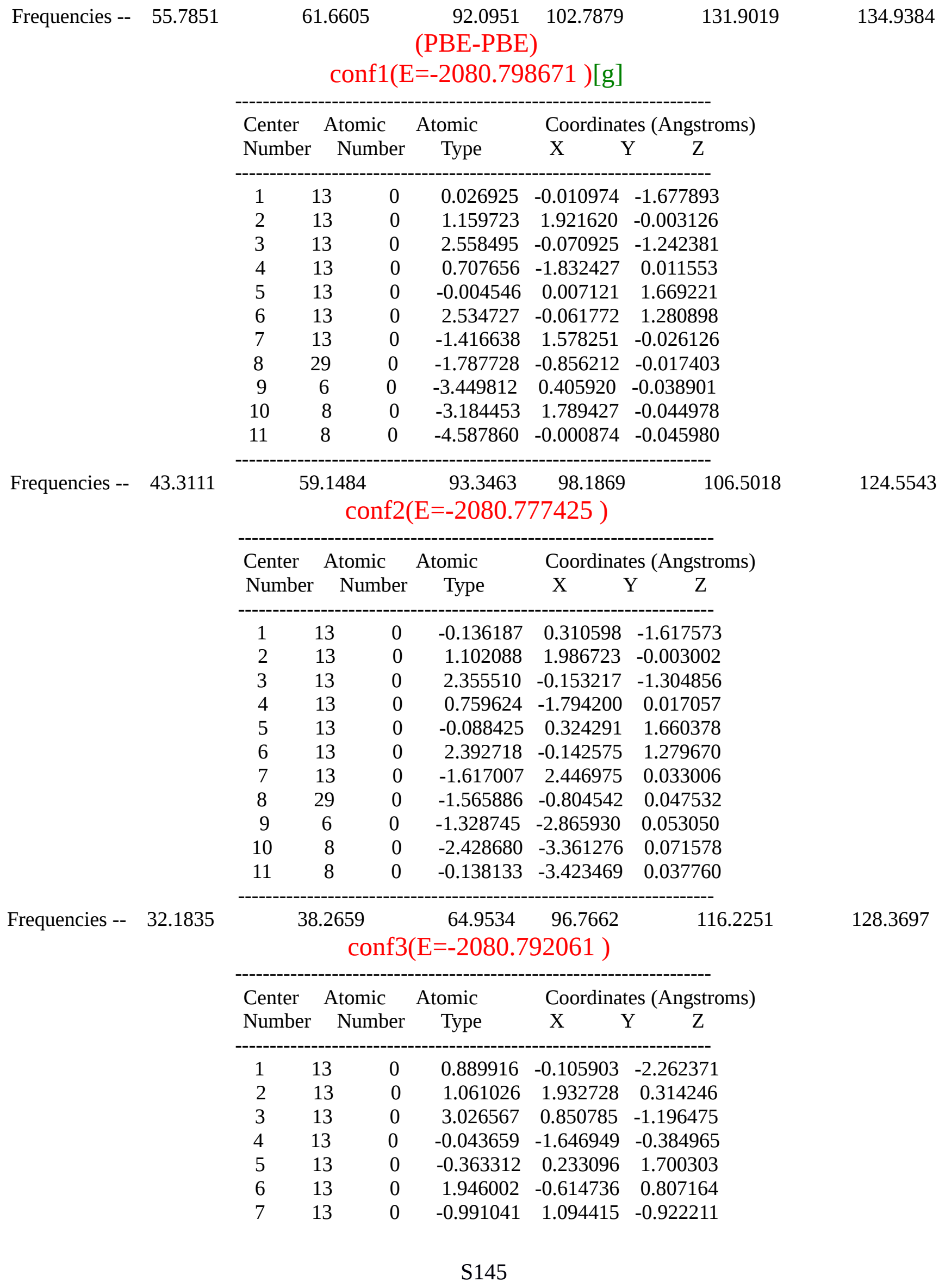




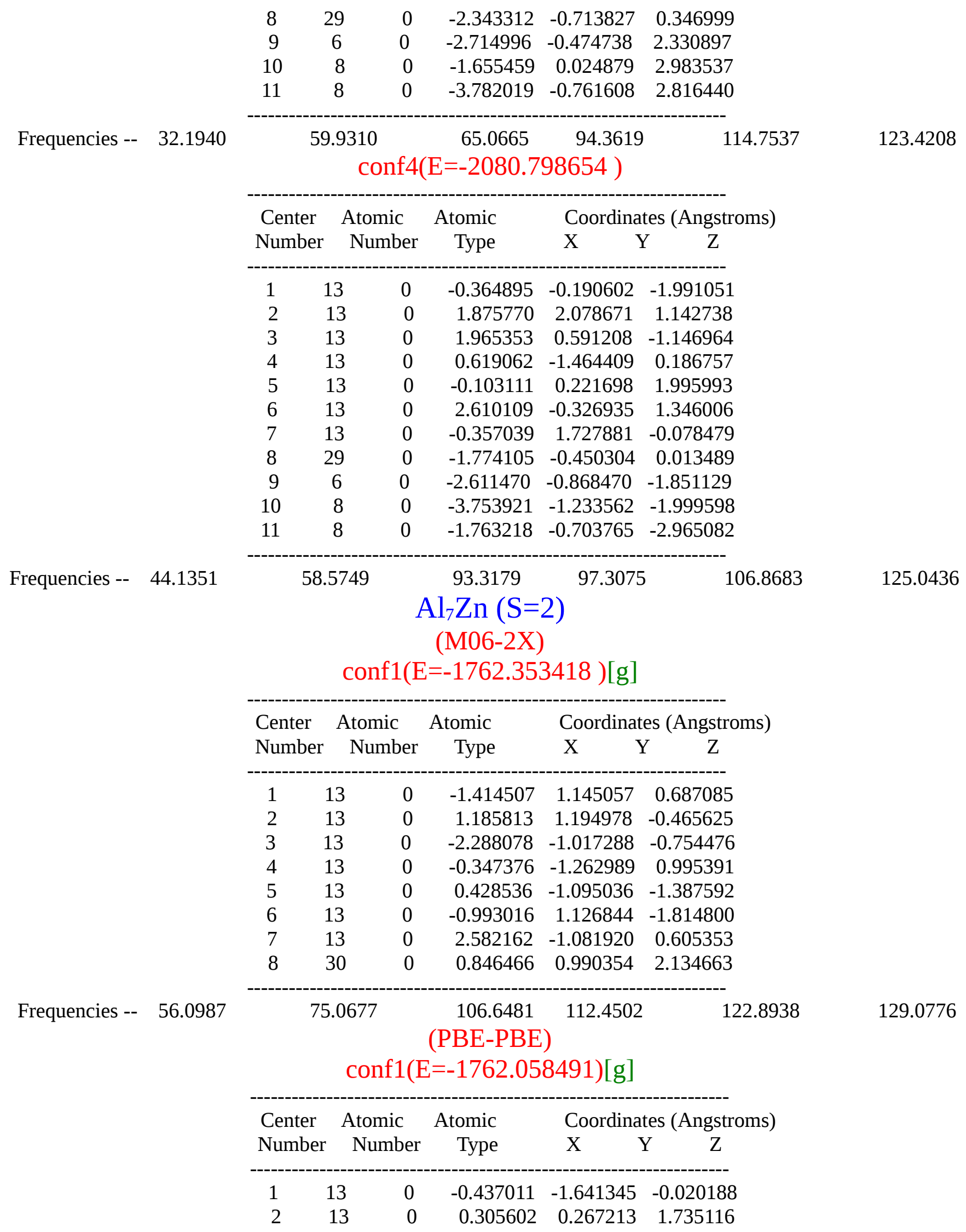




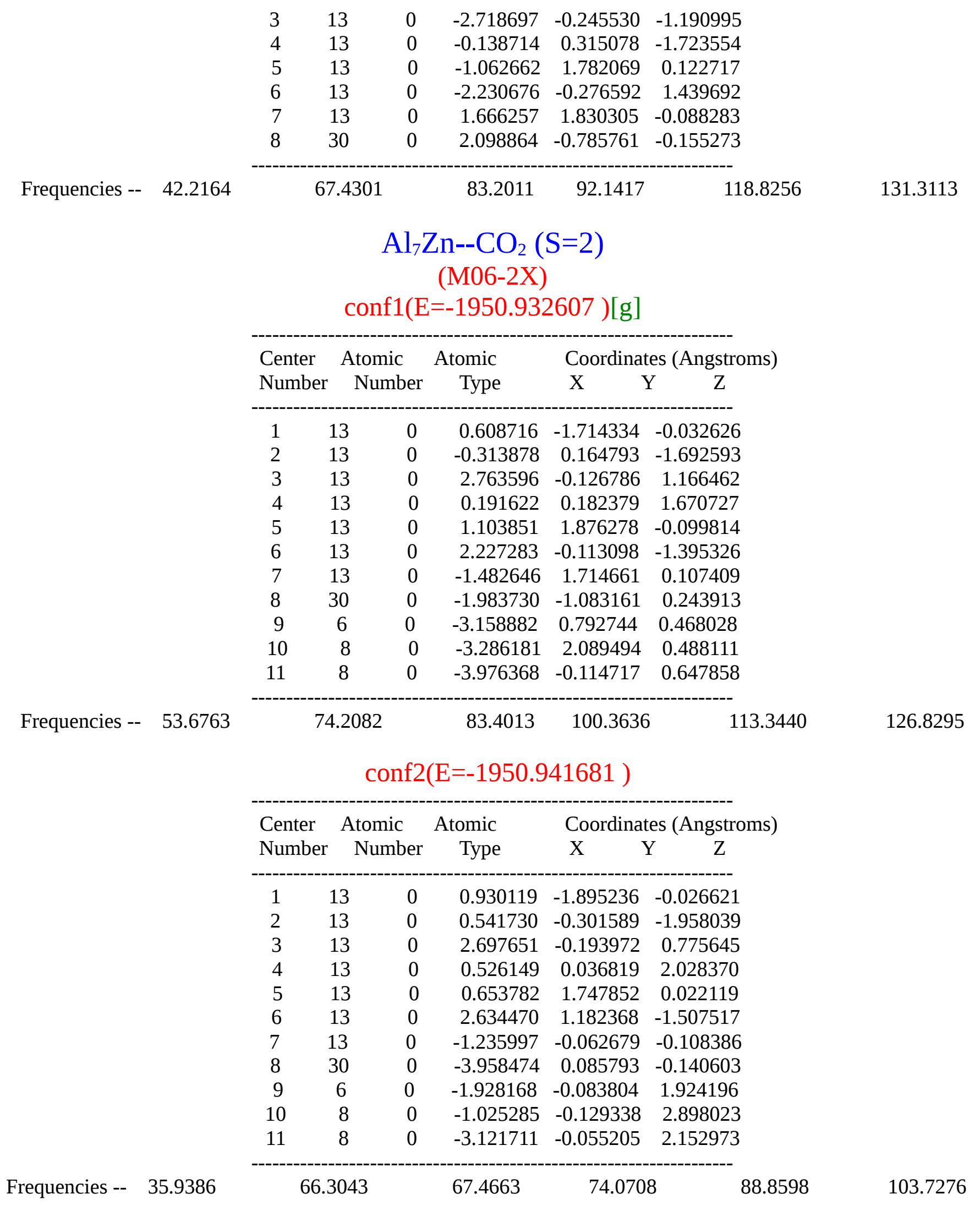




\section{$\operatorname{conf3(E=-1950.935895)}$}

\begin{tabular}{|c|c|c|c|c|c|}
\hline \multirow{2}{*}{$\begin{array}{l}\text { Center } \\
\text { Number }\end{array}$} & \multirow{2}{*}{\multicolumn{2}{|c|}{$\begin{array}{l}\text { Atomic } \\
\text { Number }\end{array}$}} & \multirow{2}{*}{$\begin{array}{r}\text { Atomic } \\
\text { Type }\end{array}$} & \multicolumn{2}{|c|}{ Coordinates (Angstro } \\
\hline & & & & $\mathrm{X}$ & $Y \quad Z$ \\
\hline 1 & 13 & 0 & 1.021505 & -2.086117 & -0.060370 \\
\hline 2 & 13 & 0 & -0.119512 & -0.057248 & -1.527300 \\
\hline 3 & 13 & 0 & 2.442292 & 0.046707 & 1.232998 \\
\hline 4 & 13 & 0 & -0.089358 & -0.100808 & 1.265401 \\
\hline 5 & 13 & 0 & 1.159689 & 1.978277 & -0.106340 \\
\hline 6 & 13 & 0 & 2.389229 & -0.306621 & -1.367423 \\
\hline 7 & 13 & 0 & -1.461082 & 1.774642 & -0.087773 \\
\hline 8 & 30 & 0 & -3.173629 & -0.156283 & 0.614621 \\
\hline 9 & 6 & 0 & -1.040505 & -2.002312 & 0.940277 \\
\hline 10 & 8 & 0 & -0.333661 & -3.068941 & 0.668998 \\
\hline 11 & 8 & 0 & -2.269090 & -2.061826 & 1.089614 \\
\hline
\end{tabular}

Frequencies -- 38.9828

$\begin{array}{ccc}73.7877 & 87.4293 & 91.5712\end{array}$

139.7974

\begin{tabular}{|c|c|c|c|c|c|c|}
\hline \multirow{2}{*}{$\begin{array}{l}\text { Center } \\
\text { Number }\end{array}$} & \multirow{2}{*}{\multicolumn{2}{|c|}{$\begin{array}{l}\text { Atomic } \\
\text { Number }\end{array}$}} & \multirow{2}{*}{$\begin{array}{r}\text { Atomic } \\
\text { Type }\end{array}$} & \multicolumn{2}{|c|}{ Coordinates (Angstroms) } & \\
\hline & & & & $\mathrm{X}$ & $Y \quad Z$ & \\
\hline 1 & 13 & 0 & 0.690352 & -1.570090 & 0.145626 & \\
\hline 2 & 13 & 0 & -0.157090 & -0.100658 & -1.928471 & \\
\hline 3 & 13 & 0 & 2.683266 & 0.163837 & 1.367751 & \\
\hline 4 & 13 & 0 & 0.049497 & 0.375503 & 1.785539 & \\
\hline 5 & 13 & 0 & 0.999783 & 1.626519 & -0.337704 & \\
\hline 6 & 13 & 0 & 2.443013 & -0.365272 & -1.361073 & \\
\hline 7 & 13 & 0 & -1.530973 & 1.403015 & -0.075681 & \\
\hline 8 & 30 & 0 & -1.844982 & -1.473960 & 0.529653 & \\
\hline 9 & 6 & 0 & -2.479920 & 0.069753 & -1.381431 & \\
\hline 10 & 8 & 0 & -1.886230 & -0.136601 & -2.539238 & \\
\hline 11 & 8 & 0 & -3.285004 & -0.770820 & -0.928810 & \\
\hline & & & 83.3084 & 113.2315 & 123.9865 & 143.6243 \\
\hline
\end{tabular}

Frequencies -- 24.3026

\section{(PBE-PBE) \\ conf1(E=-1950.519207)[g]}

Center Atomic Atomic Coordinates (Angstroms)

Number Number Type X $\quad$ Y Z

$\begin{array}{rrrrrr}1 & 6 & 0 & -1.043300 & -2.237456 & 0.353146 \\ 2 & 8 & 0 & -0.182820 & -3.158592 & 0.743940 \\ 3 & 8 & 0 & -2.276668 & -2.359557 & 0.515804 \\ 4 & 13 & 0 & 1.241390 & -1.941526 & 0.697251 \\ 5 & 13 & 0 & -0.414626 & -0.489886 & -0.772255 \\ 6 & 13 & 0 & 2.809928 & 0.176196 & 0.966526 \\ 7 & 13 & 0 & 0.404218 & 0.440244 & 1.788006\end{array}$




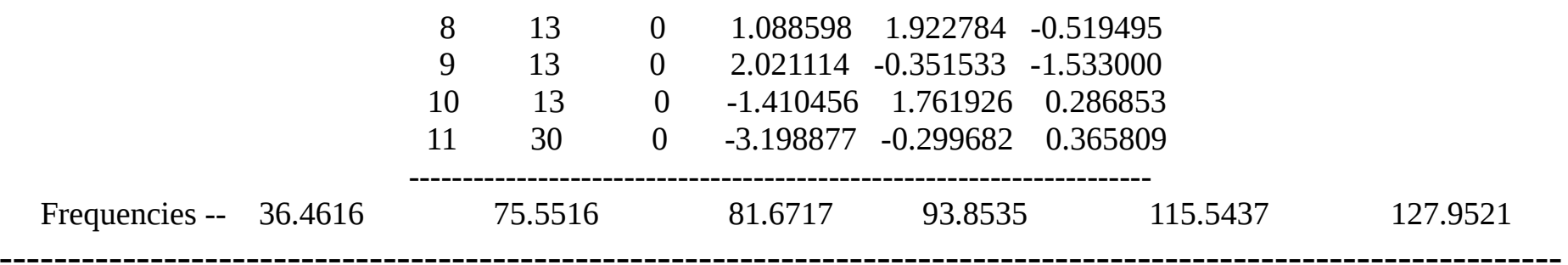




\section{Additional Informations}

1.Table S23:Wigner-Seitz radii of bulk metals

$\begin{array}{ccc}\text { First row } & \text { in Angstom } & \text { in a.u(bohr) } \\ \mathrm{Sc} & 1.814 & 3.4227 \\ \mathrm{Ti} & 1.6147 & 3.0465 \\ \mathrm{~V} & 1.4895 & 2.8104 \\ \mathrm{Cr} & 1.4238 & 2.6865 \\ \mathrm{Mn} & 1.4285 & 2.6953 \\ \mathrm{Fe} & 1.4113 & 2.6629 \\ \mathrm{Co} & 1.3794 & 2.6026 \\ \mathrm{Ni} & 1.3771 & 2.5983 \\ \mathrm{Cu} & 1.4134 & 2.6668 \\ \mathrm{Zn} & 1.5368 & 2.8996\end{array}$

2.Table S24:Data for Low spin Clusters

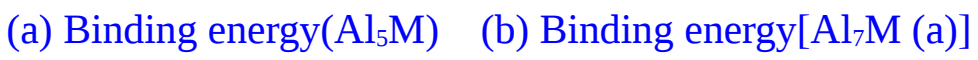

$\begin{array}{cc}\text { M062X } & \text { PBEPBE } \\ 1.722 & 2.0357 \\ 2.0216 & 2.3722 \\ 1.7354 & 2.1288 \\ 2.7038 & 3.0322 \\ 1.8941 & 2.2698 \\ 2.3916 & 2.6028 \\ 1.4751 & 2.1862 \\ 2.2121 & 2.9012 \\ 1.7598 & 1.9585 \\ 1.4615 & 1.5911\end{array}$

$\begin{array}{cc}\mathrm{M} 062 \mathrm{X} & \mathrm{PBEPBE} \\ 2.0032 & 2.2663 \\ 2.2574 & 2.5358 \\ 1.989 & 2.2512 \\ 2.4753 & 3.0238 \\ 2.1078 & 2.2549 \\ 2.6246 & 2.752 \\ 1.7945 & 2.4107 \\ 2.3887 & 2.9104 \\ 2.0645 & 2.2241 \\ 1.8254 & 1.9529\end{array}$

(c)

$\begin{array}{cc}\text { c) Binding energy }\left[\mathrm{Al}_{7} \mathrm{M}(\mathrm{b})\right] \\ \text { M062X } & \text { PBEPBE } \\ 2.0165 & 2.2729 \\ 2.2888 & 2.5399 \\ 2.0694 & 2.3761 \\ 2.5144 & 3.0056 \\ 2.176 & 2.3249 \\ 2.4605 & 2.752 \\ 1.8552 & 2.4107 \\ 2.4192 & 2.98 \\ 2.0721 & 2.232 \\ 1.8129 & 1.9212\end{array}$

(d) Binding energy[ $\mathrm{Al}_{7} \mathrm{M}$ (c)]

$\begin{array}{cc}\text { M062X } & \text { PBEPBE } \\ 2.0268 & 2.2846 \\ 2.1671 & 2.541 \\ 2.0694 & 2.3761 \\ 2.6349 & 3.0238 \\ 2.0509 & 2.3187 \\ 2.2982 & 2.6312 \\ 1.8552 & 2.4107 \\ 2.4192 & 2.98 \\ 1.9551 & 2.1409 \\ 1.8129 & 1.9212\end{array}$

(e) Distance of transition metal from cluster center

(f) Ionization potential of $\mathrm{Al}_{5} \mathrm{M}$ clusters

$\begin{array}{ccc} & \text { M062X } & \text { PBEPBE } \\ \mathrm{Sc} & 1.78818 & 1.81877 \\ \mathrm{Ti} & 1.8524 & 1.76757 \\ \mathrm{~V} & 1.32 & 0.81569 \\ \mathrm{Cr} & 1.817 & 1.25491 \\ \mathrm{Mn} & 1.33 & 0.60841 \\ \mathrm{Fe} & 1.59 & 1.38169 \\ \mathrm{Co} & 1.09 & 0.82602 \\ \mathrm{Ni} & 1.26 & 0.99 \\ \mathrm{Cu} & 1.21 & 1.188 \\ \mathrm{Zn} & 1.9 & 1.87\end{array}$

$\begin{array}{cccc} & \mathrm{E}_{(\mathrm{N}-1)} & \mathrm{E}_{\mathrm{N}} & \mathrm{IP}(\mathrm{in} \mathrm{eV}) \\ \mathrm{Sc} & -1258.165894 & -1258.379345 & 5.810659996 \\ \mathrm{Ti} & -1269.732393 & -1269.971707 & 5.83574946 \\ \mathrm{~V} & -1283.023188 & -1283.182988 & 4.165993928 \\ \mathrm{Cr} & -1297.91155 & -1298.291787 & 10.08463114 \\ \mathrm{Mn} & -1315.698484 & -1315.778153 & 2.295794804 \\ \mathrm{Fe} & -1335.09563 & -1335.417505 & 8.263141496 \\ \mathrm{Co} & -1356.851259 & -1357.022742 & 4.148959216 \\ \mathrm{Ni} & -1381.104418 & -1381.355267 & 6.82640232 \\ \mathrm{Cu} & -1408.018959 & -1408.24351 & 6.109855936 \\ \mathrm{Zn} & -1277.239276 & -1277.460165 & 6.010749832\end{array}$


3.Table S25: Data for High spin Clusters

(a) Binding energies $\left(\mathrm{Al}_{5} \mathrm{M}\right)$

$\begin{array}{cc} & \text { M062X } \\ \mathrm{Sc} & 1.7572 \\ \mathrm{Ti} & 2.0521 \\ \mathrm{~V} & 2.1129 \\ \mathrm{Cr} & 2.7871 \\ \mathrm{Mn} & 2.5723 \\ \mathrm{Fe} & 2.4508 \\ \mathrm{Co} & 1.805 \\ \mathrm{Ni} & 2.2121 \\ \mathrm{Cu} & 1.7598 \\ \mathrm{Zn} & 1.4615\end{array}$

(b) Distance of M from cluster center

$\begin{array}{cccc} & \text { M062X } & \text { PBEPBE } & \text { WS(ang) } \\ \mathrm{Sc} & 1.865 & 1.87 & 1.814 \\ \mathrm{Ti} & 1.87684 & 1.79338 & 1.6147 \\ \mathrm{~V} & 1.48 & 1.18 & 1.4895 \\ \mathrm{Cr} & 1.545 & 1.31564 & 1.4238 \\ \mathrm{Mn} & 1.73862 & 1.65115 & 1.4285 \\ \mathrm{Fe} & 1.78225 & 1.53802 & 1.4113 \\ \mathrm{Co} & 1.369 & 0.82 & 1.3794 \\ \mathrm{Ni} & 1.26 & 0.99 & 1.3771 \\ \mathrm{Cu} & 1.21 & 1.188 & 1.4134 \\ \mathrm{Zn} & 1.9 & 1.87 & 1.5368\end{array}$

(c) NICS value of $\mathrm{Al}_{5} \mathrm{M}$ clusters

-NICS

Values

M062X

Sc

Ti

$\mathrm{V}$

$\mathrm{Cr}$

Mn

$\mathrm{Fe}$

Co

$\mathrm{Ni}$

$\mathrm{Cu}$

$\mathrm{Zn}$
$-188.6614$

$-53.1527$

$-63.676$

4.0114

$-24.819$

0.1091

156.7572

15.756

4.0291

$-17.8404$
PBEPBE

1.9235

18.2977

7.9825

20.8952

0.389

17.4738

27.7191

11.8092

6.5595

5.2094

(d) HOMO-LUMO gap for $\mathrm{Al}_{5} \mathrm{M}(\mathrm{eV})$

$\begin{array}{ccc} & \text { M062X } & \text { PBEPBE } \\ \mathrm{Sc} & 2.58949392 & 0.33661244 \\ \mathrm{Ti} & 2.75222168 & 0.34042212 \\ \mathrm{~V} & 2.81426504 & 0.56301628 \\ \mathrm{Cr} & 2.86923328 & 0.66506128 \\ \mathrm{Mn} & 2.66051724 & 0.44981436 \\ \mathrm{Fe} & 2.8314086 & 0.5673702 \\ \mathrm{Co} & 2.91467732 & 0.6149912 \\ \mathrm{Ni} & 2.79630512 & 0.46450884 \\ \mathrm{Cu} & 2.92311304 & 0.60492276 \\ \mathrm{Zn} & 2.70813824 & 0.53389944\end{array}$


Ionization Potential for $\mathrm{Al}_{5} \mathrm{M}$ cluster

$\begin{array}{ll}\mathrm{Sc} & 6.011566192 \\ \mathrm{Ti} & 5.936488284 \\ \mathrm{~V} & 6.017879376 \\ \mathrm{Cr} & 6.007157848 \\ \mathrm{Mn} & 5.978558036 \\ \mathrm{Fe} & 6.198539844 \\ \mathrm{Co} & 6.129829544 \\ \mathrm{Ni} \text { (cation is triplet) } & 6.129992816 \\ \mathrm{Cu} & 6.109855936 \\ \mathrm{Zn} & 6.010749832\end{array}$

(e)

Ionization Potential of elements (NIST)

$\begin{array}{cc} & \text { IP elem }(\mathrm{eV}) \\ \mathrm{Sc} & 6.56149 \\ \mathrm{Ti} & 6.82812 \\ \mathrm{~V} & 6.746187 \\ \mathrm{Cr} & 6.76651 \\ \mathrm{Mn} & 7.434018 \\ \mathrm{Fe} & 7.9024678 \\ \mathrm{Co} & 7.88101 \\ \mathrm{Ni} & 7.639877 \\ \mathrm{Cu} & 7.72638 \\ \mathrm{Zn} & 9.394199\end{array}$


Thermodynamic Data of $\mathrm{CO}_{2}$ Adsortption on Doped aluminum clusters in PBE-PBE functional

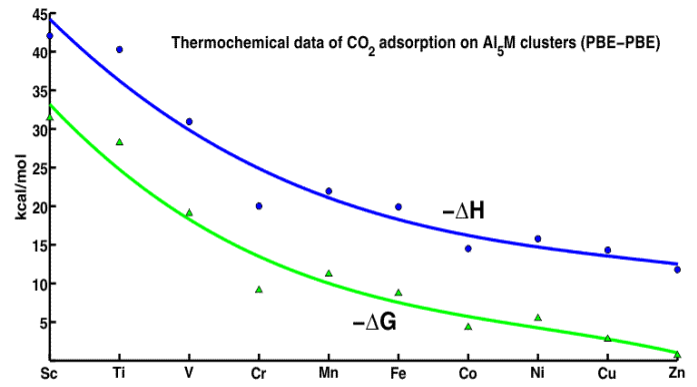

(a)

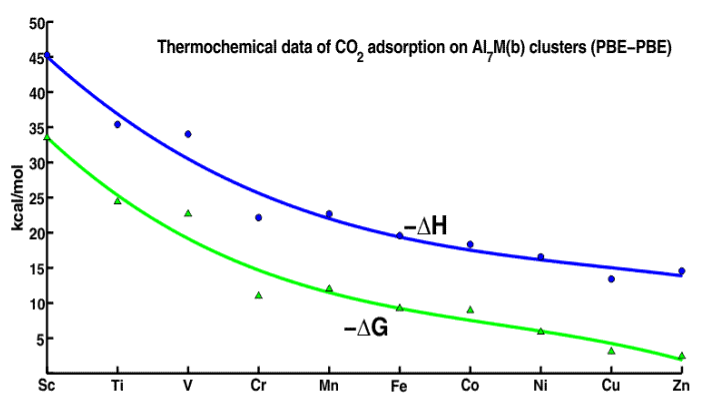

(c)

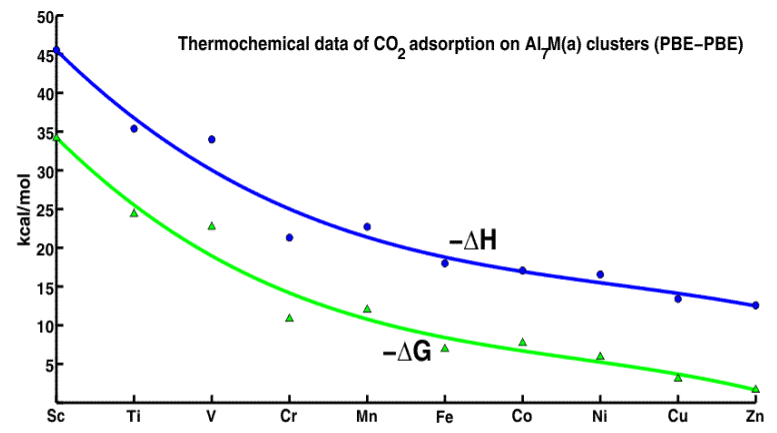

(b)

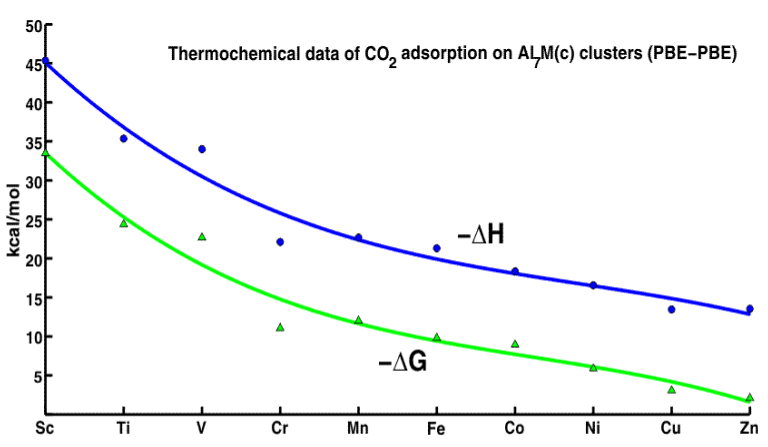

(d)

Figure S7: Plots of thermochemical properties $(-\Delta \mathrm{H}$ and $-\Delta \mathrm{G})$ of adsorption of $\mathrm{CO}_{2}$ on transition metal doped aluminum clusters in PBE-PBE functional (a) $\mathrm{Al}_{5} \mathrm{M}$.... $\mathrm{CO}_{2}$ (b)-(d) $\mathrm{Al}_{7} \mathrm{M}[(\mathrm{a})-(\mathrm{c})] \ldots \mathrm{CO}_{2}$. 

D) PBE-PBE functional.

(i) $\mathrm{Al}_{5} \mathrm{M}$ Cluster

\begin{tabular}{|c|c|c|c|c|c|c|}
\hline Dopant & Parameters & Cluster+CO2 & doped cluster & $\mathrm{CO} 2$ & Delta(in a.u.) & in $\mathrm{Kcal} / \mathrm{mol}$ \\
\hline Sc & $\mathrm{U}$ & -1446.437847 & -1257.925118 & -188.439942 & -0.072787 & -45.67457037 \\
\hline \multirow[t]{4}{*}{3} & U+therm & -1446.425195 & -1257.915569 & -188.437289 & -0.072337 & -45.39219087 \\
\hline & $\mathrm{H}$ & -1446.424251 & -1257.914625 & -188.436345 & -0.073281 & -45.98456031 \\
\hline & $\mathrm{G}$ & -1446.479009 & -1257.962249 & -188.460668 & -0.056092 & -35.19829092 \\
\hline & & & & & 0 & 0 \\
\hline $\mathrm{Ti}$ & $\mathrm{U}$ & -1458.045854 & -1269.536783 & -188.439942 & -0.069129 & -43.37913879 \\
\hline \multirow[t]{4}{*}{4} & U+therm & -1458.033195 & -1269.526888 & -188.437289 & -0.069018 & -43.30948518 \\
\hline & $\mathrm{H}$ & -1458.032251 & -1269.525943 & -188.436345 & -0.069963 & -43.90248213 \\
\hline & $G$ & -1458.087403 & -1269.57603 & -188.460668 & -0.050705 & -31.81789455 \\
\hline & & & & & 0 & 0 \\
\hline V & $\mathrm{U}$ & -1471.292563 & -1282.797969 & -188.439942 & -0.054652 & -34.29467652 \\
\hline \multirow[t]{4}{*}{5} & U+therm & -1471.279952 & -1282.78791 & -188.437289 & -0.054753 & -34.35805503 \\
\hline & $\mathrm{H}$ & -1471.279008 & -1282.786966 & -188.436345 & -0.055697 & -34.95042447 \\
\hline & $G$ & -1471.334306 & -1282.837465 & -188.460668 & -0.036173 & -22.69891923 \\
\hline & & & & & 0 & 0 \\
\hline $\mathrm{Cr}$ & $\mathrm{U}$ & -1486.270848 & -1297.793348 & -188.439942 & -0.037558 & -23.56802058 \\
\hline \multirow[t]{4}{*}{6} & U+therm & -1486.257855 & -1297.783253 & -188.437289 & -0.037313 & -23.41428063 \\
\hline & $\mathrm{H}$ & -1486.256911 & -1297.782309 & -188.436345 & -0.038257 & -24.00665007 \\
\hline & G & -1486.314137 & -1297.833225 & -188.460668 & -0.020244 & -12.70331244 \\
\hline & & & & & 0 & $\begin{array}{l}0 \\
\end{array}$ \\
\hline Mn & $\mathrm{U}$ & -1503.875411 & -1315.398497 & -188.439942 & -0.036972 & -23.20029972 \\
\hline \multirow[t]{4}{*}{7} & U+therm & -1503.862192 & -1315.38859 & -188.437289 & -0.036313 & -22.78677063 \\
\hline & $\mathrm{H}$ & -1503.861248 & -1315.387646 & -188.436345 & -0.037257 & -23.37914007 \\
\hline & $G$ & -1503.9191 & -1315.437699 & -188.460668 & -0.020733 & -13.01016483 \\
\hline & & & & & 0 & \begin{tabular}{|l|}
0 \\
\end{tabular} \\
\hline $\mathrm{Fe}$ & $\mathrm{U}$ & -1523.409566 & -1334.933412 & -188.439942 & -0.036212 & -22.72339212 \\
\hline \multirow[t]{4}{*}{4} & U+therm & -1523.396638 & -1334.923476 & -188.437289 & -0.035873 & -22.51066623 \\
\hline & $\mathrm{H}$ & -1523.395694 & -1334.922532 & -188.436345 & -0.036817 & -23.10303567 \\
\hline & G & -1523.452327 & -1334.972508 & -188.460668 & -0.019151 & -12.01744401 \\
\hline & & & & & 0 & 0 \\
\hline $\mathrm{Co}$ & $\mathrm{U}$ & -1545.068494 & -1356.599939 & -188.439942 & -0.028613 & -17.95494363 \\
\hline \multirow[t]{4}{*}{3} & U+therm & -1545.055397 & -1356.590049 & -188.437289 & -0.028059 & -17.60730309 \\
\hline & $\mathrm{H}$ & -1545.054453 & -1356.589105 & -188.436345 & -0.029003 & -18.19967253 \\
\hline & G & -1545.11106 & -1356.637933 & -188.460668 & -0.012459 & -7.81814709 \\
\hline & & & & & 0 & 0 \\
\hline $\mathrm{Ni}$ & $\mathrm{U}$ & -1569.312356 & -1380.840832 & -188.439942 & -0.031582 & -19.81802082 \\
\hline \multirow[t]{4}{*}{2} & U+therm & -1569.299393 & -1380.831025 & -188.437289 & -0.031079 & -19.50238329 \\
\hline & $\mathrm{H}$ & -1569.298449 & -1380.830081 & -188.436345 & -0.032023 & -20.09475273 \\
\hline & $\mathrm{G}$ & -1569.354027 & -1380.878513 & -188.460668 & -0.014846 & -9.31601346 \\
\hline & & & & & 0 & \begin{tabular}{|l|}
0 \\
\end{tabular} \\
\hline $\mathrm{Cu}$ & $\mathrm{U}$ & -1596.131276 & -1407.662561 & -188.439942 & -0.028773 & -18.05534523 \\
\hline \multirow[t]{4}{*}{1} & U+therm & -1596.118498 & -1407.652516 & -188.437289 & -0.028693 & -18.00514443 \\
\hline & $\mathrm{H}$ & -1596.117554 & -1407.651572 & -188.436345 & -0.029637 & -18.59751387 \\
\hline & G & -1596.171923 & -1407.700813 & -188.460668 & -0.010442 & -6.55245942 \\
\hline & & & & & 0 & 0 \\
\hline $\mathrm{Zn}$ & $\mathrm{U}$ & -1465.86414 & -1277.405243 & -188.439942 & -0.018955 & -11.89445205 \\
\hline \multirow[t]{3}{*}{2} & U+therm & -1465.850813 & -1277.395117 & -188.437289 & -0.018407 & -11.55057657 \\
\hline & $\mathrm{H}$ & -1465.849868 & -1277.394173 & -188.436345 & -0.01935 & -12.1423185 \\
\hline & $\mathrm{G}$ & -1465.908558 & -1277.444123 & -188.460668 & -0.003767 & -2.36383017 \\
\hline
\end{tabular}


(ii) $\mathrm{Al}_{7} \mathrm{M}$ (c) Cluster

\begin{tabular}{|c|c|c|c|c|c|c|}
\hline & & bped cluster+CC & doped cluster & $\mathrm{CO} 2$ & orption energy & in Kcal/mol \\
\hline Sc & $\mathrm{U}$ & -1931.124069 & -1742.608369 & -188.439942 & -0.075758 & -47.53890258 \\
\hline \multirow[t]{4}{*}{3} & U+therm & -1931.108606 & -1742.595593 & -188.437289 & -0.075724 & -47.51756724 \\
\hline & $\mathrm{H}$ & -1931.107662 & -1742.594649 & -188.436345 & -0.076668 & -48.10993668 \\
\hline & G & -1931.168107 & -1742.649649 & -188.460668 & -0.05779 & -36.2638029 \\
\hline & & & & & 0 & 0 \\
\hline $\mathrm{Ti}$ & $\mathrm{U}$ & -1942.728972 & -1754.227968 & -188.439942 & -0.061062 & -38.31701562 \\
\hline \multirow[t]{4}{*}{4} & U+therm & -1942.713323 & -1754.215336 & -188.437289 & -0.060698 & -38.08860198 \\
\hline & $\mathrm{H}$ & -1942.712379 & -1754.214392 & -188.436345 & -0.061642 & -38.68097142 \\
\hline & $\mathrm{G}$ & -1942.773725 & -1754.268853 & -188.460668 & -0.044204 & -27.73845204 \\
\hline & & & & & 0 & 0 \\
\hline $\mathrm{V}$ & $\mathrm{U}$ & -1955.983843 & -1767.485271 & -188.439942 & -0.05863 & -36.7909113 \\
\hline \multirow[t]{4}{*}{5} & U+therm & -1955.968203 & -1767.472484 & -188.437289 & -0.05843 & -36.6654093 \\
\hline & $\mathrm{H}$ & -1955.967259 & -1767.47154 & -188.436345 & -0.059374 & -37.25777874 \\
\hline & $\mathrm{G}$ & -1956.028915 & -1767.526857 & -188.460668 & -0.04139 & -25.9726389 \\
\hline & & & & & 0 & 0 \\
\hline $\mathrm{Cr}$ & $\mathrm{U}$ & -1970.962067 & -1782.481686 & -188.439942 & -0.040439 & -25.37587689 \\
\hline \multirow[t]{4}{*}{6} & U+therm & -1970.946095 & -1782.468701 & -188.437289 & -0.040105 & -25.16628855 \\
\hline & $\mathrm{H}$ & -1970.945151 & -1782.467757 & -188.436345 & -0.041049 & -25.75865799 \\
\hline & $\mathrm{G}$ & -1971.008349 & -1782.524099 & -188.460668 & -0.023582 & -14.79794082 \\
\hline & & & & & 0 & 0 \\
\hline $\mathrm{Mn}$ & $\mathrm{U}$ & -1988.574567 & -1800.093892 & -188.439942 & -0.040733 & -25.56036483 \\
\hline \multirow[t]{4}{*}{5} & U+therm & -1988.558539 & -1800.081045 & -188.437289 & -0.040205 & -25.22903955 \\
\hline & $\mathrm{H}$ & -1988.557595 & -1800.080101 & -188.436345 & -0.041149 & -25.82140899 \\
\hline & G & -1988.62037 & -1800.135761 & -188.460668 & -0.023941 & -15.02321691 \\
\hline & & & & & 0 & 0 \\
\hline $\mathrm{Fe}$ & $\mathrm{U}$ & -2008.100325 & -1819.621757 & -188.439942 & -0.038626 & -24.23820126 \\
\hline \multirow[t]{4}{*}{4} & U+therm & -2008.084392 & $\mid-1819.609071$ & -188.437289 & -0.038032 & -23.86546032 \\
\hline & $\mathrm{H}$ & -2008.083448 & $\mid-1819.608127$ & -188.436345 & -0.038976 & -24.45782976 \\
\hline & $G$ & -2008.145781 & -1819.662864 & -188.460668 & -0.022249 & -13.96146999 \\
\hline & & & & & 0 & 0 \\
\hline Co & $\mathrm{U}$ & -2029.755599 & -1841.280664 & -188.439942 & -0.034993 & -21.95845743 \\
\hline \multirow[t]{4}{*}{3} & U+therm & -2029.739646 & -1841.267794 & -188.437289 & -0.034563 & -21.68862813 \\
\hline & $\mathrm{H}$ & -2029.738702 & $\mid-1841.266849$ & -188.436345 & -0.035508 & -22.28162508 \\
\hline & $\mathrm{G}$ & -2029.801311 & $\mid-1841.322196$ & -188.460668 & -0.018447 & -11.57567697 \\
\hline & & & & & 0 & 0 \\
\hline $\mathrm{Ni}$ & $\mathrm{U}$ & -2054.010076 & -1865.539225 & -188.439942 & -0.030909 & -19.39570659 \\
\hline \multirow[t]{4}{*}{2} & U+therm & -2053.994244 & -1865.526621 & -188.437289 & -0.030334 & -19.03488834 \\
\hline & $\mathrm{H}$ & -2053.9933 & -1865.525676 & -188.436345 & -0.031279 & -19.62788529 \\
\hline & G & -2054.054946 & -1865.58047 & -188.460668 & -0.013808 & -8.66465808 \\
\hline & & & & & 0 & 0 \\
\hline $\mathrm{Cu}$ & $\mathrm{U}$ & -2080.818665 & -1892.353158 & -188.439942 & -0.025565 & -16.04229315 \\
\hline \multirow[t]{4}{*}{1} & U+therm & -2080.802379 & -1892.340132 & -188.437289 & -0.024958 & -15.66139458 \\
\hline & $\mathrm{H}$ & -2080.801434 & -1892.339188 & -188.436345 & -0.025901 & -16.25313651 \\
\hline & $\mathrm{G}$ & -2080.864426 & -1892.394344 & -188.460668 & -0.009414 & -5.90737914 \\
\hline & & & & & 0 & 0 \\
\hline & $\mathrm{U}$ & -1950.541395 & -1762.075985 & -188.439942 & -0.025468 & -15.98142468 \\
\hline \multirow[t]{3}{*}{2} & U+therm & -1950.525028 & -1762.062536 & -188.437289 & -0.025203 & -15.81513453 \\
\hline & $\mathrm{H}$ & -1950.524084 & -1762.061592 & -188.436345 & -0.026147 & -16.40750397 \\
\hline & G & -1950.58778 & -1762.119576 & -188.460668 & -0.007536 & -4.72891536 \\
\hline & & & & & & \\
\hline & & & & & & \\
\hline
\end{tabular}




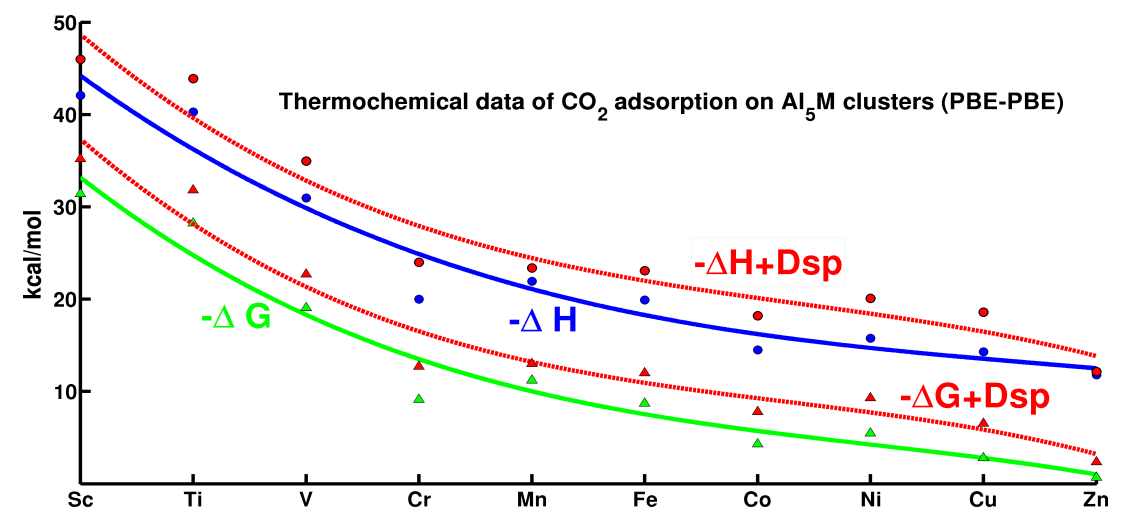

(a)

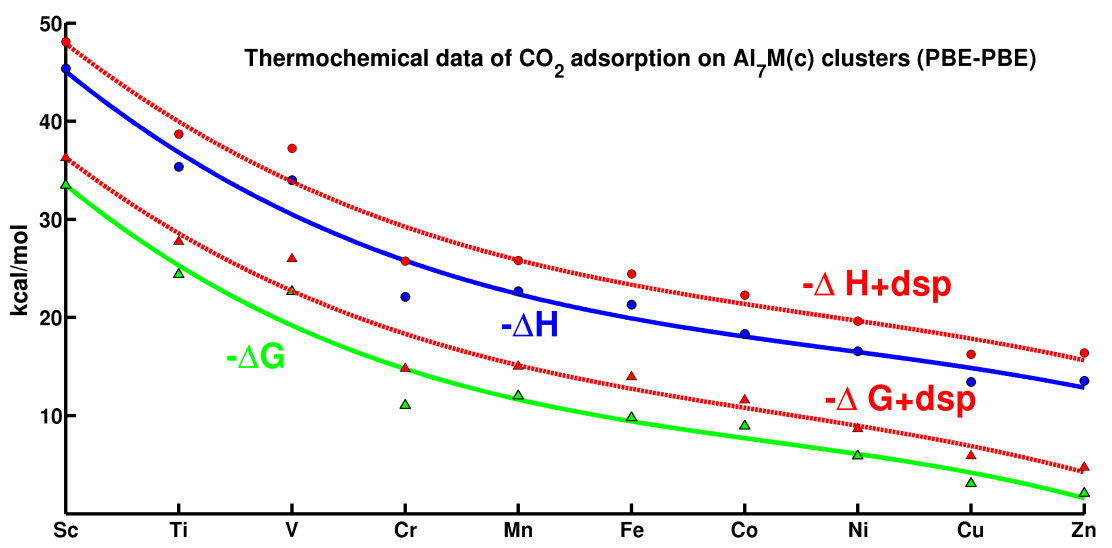

(b)

Figure S8: Comparison of thermodynamic data calculated in PBE-PBE and dispersion corrected PBE-PBE functional (red line) for (a) $\mathrm{Al}_{5} \mathrm{M}$ cluster and (b) $\mathrm{Al}_{7} \mathrm{M}$ (c) cluster. From both the plot it is evident that dispersion effect is less significant for aluminum-CO2 complex as the difference is found to be small( $\sim|0.3|-|4.3| \mathrm{kcal} / \mathrm{mol}$ which is well within the DFT error range). The overall trends in the thermodynamic parameters are also preserved and no significant change is observed. 
Cartesian Coordinates and Normal Modes of Vibrations of $\mathrm{Al}_{5} \underline{\mathrm{M}}$ and $\mathrm{Al}_{2} \mathrm{M}$ (c) clusters and $\mathrm{CO} 2$ complexes in dispersion corrected PBE-PBE functional

(Only global minima are included. Coordinate value for only parallel binding modes are included. First six normal modes of vibrations are reported for each case. Energy values are given in a.u)

(A)Impure clusters of $\mathrm{Al}_{5} \underline{\underline{\mathrm{M}} \text { series }}$

$\mathrm{Al}_{5} \mathrm{Sc}(\mathrm{S}=3)$

$(\mathrm{E}=-1257.925118)$

\begin{tabular}{rrrrrr}
\multicolumn{2}{c}{ Center } & \multicolumn{2}{c}{ Atomic } & Atomic & \multicolumn{3}{c}{ Coordinates } & Angstr \\
Number & Number & Type & X & Y & Z \\
\hline 1 & 13 & 0 & 0.202783 & 0.098518 & 1.867282 \\
2 & 13 & 0 & 0.203868 & -1.869021 & -0.029290 \\
3 & 13 & 0 & 2.173346 & -0.000553 & 0.001867 \\
4 & 13 & 0 & 0.204986 & 1.869027 & 0.029609 \\
5 & 13 & 0 & 0.206140 & -0.098543 & -1.867003 \\
6 & 21 & 0 & -1.891350 & 0.000648 & -0.001764
\end{tabular}

Frequencies -- 76.1681

$\begin{array}{ll}119.6648 & 135.9265 \\ 193.8002 & 153.1365\end{array}$

189.8309

$\mathrm{Al}_{5} \mathrm{Sc}--\mathrm{CO}_{2}(\mathrm{~S}=3)$

$(\mathrm{E}=-1446.437847)$

\begin{tabular}{|c|c|c|c|c|c|}
\hline \multirow{2}{*}{$\begin{array}{l}\text { Center } \\
\text { Number }\end{array}$} & \multicolumn{2}{|c|}{ Atomic } & \multirow{2}{*}{$\begin{array}{l}\text { Atomic } \\
\text { Type }\end{array}$} & \multicolumn{2}{|c|}{ Coordinates (Angstroms) } \\
\hline & & Number & & $\mathrm{X} \quad \mathrm{I}-\mathrm{r}$ & $\mathrm{Y} \quad \mathrm{Z}$ \\
\hline 1 & 13 & 0 & -0.549991 & 1.516911 & 0.000003 \\
\hline 2 & 13 & 0 & 0.959068 & 0.035832 & 1.769538 \\
\hline 3 & 13 & 0 & 1.978354 & 1.818415 & 0.000001 \\
\hline 4 & 13 & 0 & 0.959064 & 0.035841 & -1.769543 \\
\hline 5 & 13 & 0 & 2.079018 & -1.549550 & -0.000008 \\
\hline 6 & 21 & 0 & -0.729060 & -1.440752 & -0.000004 \\
\hline 7 & 6 & 0 & -2.137352 & 0.208980 & 0.000003 \\
\hline 8 & 8 & 0 & -2.514524 & 1.438344 & 0.000006 \\
\hline 9 & 8 & 0 & -2.792166 & -0.876972 & 0.000001 \\
\hline
\end{tabular}


Frequencies -- 58.4143

88.4107

106.8273

119.0717

136.0743

149.1267

\section{$\mathrm{Al}_{5} \mathrm{Ti}(\mathrm{S}=4)$ \\ $(\mathrm{E}=-1269.536783)$}

Center Atomic Atomic Coordinates (Angstroms)

$\begin{array}{cccccc}\text { Number } & \text { Number } & \text { Type } & \text { X } & Y & \text { Z } \\ -1 & 13 & 0 & 0.384398 & 1.158965 & -1.633907 \\ 2 & 13 & 0 & 0.005697 & 1.421593 & 0.949797 \\ 3 & 13 & 0 & 2.247578 & 0.109224 & 1.788267 \\ 4 & 13 & 0 & 0.325546 & -1.457380 & -1.388890 \\ 5 & 13 & 0 & -0.057978 & -1.224902 & 1.197687 \\ 6 & 22 & 0 & -1.763133 & -0.006051 & -0.484842\end{array}$

Frequencies -- 31.4315

45.7473

135.2757

146.9003

166.5891

$$
\begin{gathered}
\mathrm{Al}_{5} \mathrm{Ti}--\mathrm{CO}_{2}(\mathrm{~S}=4) \\
(\mathrm{E}=-1458.045854)
\end{gathered}
$$

\begin{tabular}{|c|c|c|c|c|c|}
\hline \multirow{2}{*}{$\begin{array}{l}\text { Center } \\
\text { Number }\end{array}$} & \multirow{2}{*}{\multicolumn{2}{|c|}{$\begin{array}{l}\text { Atomic } \\
\text { Number }\end{array}$}} & \multirow{2}{*}{$\begin{array}{l}\text { Atomic } \\
\text { Type }\end{array}$} & \multicolumn{2}{|c|}{ Coordinates (Angstro } \\
\hline & & & & $\mathrm{X}$ & $\mathrm{Y} \quad \mathrm{Z}$ \\
\hline 1 & 13 & 0 & 0.958312 & 0.841017 & -1.328128 \\
\hline 2 & 13 & 0 & 0.223553 & 1.371236 & 1.292819 \\
\hline 3 & 13 & 0 & 2.394581 & 0.053306 & 0.880847 \\
\hline 4 & 13 & 0 & 0.418967 & -1.787888 & -1.476464 \\
\hline 5 & 13 & 0 & 0.081274 & -1.398239 & 1.161584 \\
\hline 6 & 22 & 0 & -1.405078 & 0.005985 & -0.504284 \\
\hline 7 & 6 & 0 & -1.775130 & 1.588156 & 0.754326 \\
\hline 8 & 8 & 0 & -2.873246 & 1.386350 & 0.163081 \\
\hline 9 & 8 & 0 & -1.389731 & 2.431480 & 1.645746 \\
\hline
\end{tabular}

Frequencies -- 55.7330

79.7572

88.3016

137.7358

146.0401

155.1063

$$
\mathrm{Al}_{5} \mathrm{~V}(\mathrm{~S}=5)
$$

$(E=-1282.797969)$

Center Atomic Atomic Coordinates (Angstroms) 


\begin{tabular}{rrrrrr} 
Number & Number & \multicolumn{1}{c}{ Type } & \multicolumn{1}{c}{ X } & \multicolumn{1}{c}{$Y$} & $\mathrm{Z}$ \\
- \hdashline 1 & 13 & 0 & 1.406576 & 1.030364 & 1.559208 \\
2 & 13 & 0 & 0.922708 & -1.519720 & 1.322002 \\
3 & 13 & 0 & 0.993404 & 1.064681 & -1.013431 \\
4 & 13 & 0 & -1.462278 & 0.692952 & -2.057760 \\
5 & 13 & 0 & 0.117926 & -1.420943 & -1.228195 \\
6 & 23 & 0 & -0.994151 & 0.153011 & 0.582804
\end{tabular}

Frequencies --

29.1059

74.6236

$121.7848 \quad 133.7502$

145.1620

\begin{tabular}{|c|c|c|c|c|c|}
\hline \multicolumn{6}{|c|}{$\begin{array}{l}\mathrm{Al}_{5} \mathrm{~V}-\mathrm{CO}_{2}(\mathrm{~S}=5) \\
(\mathrm{E}=-1471.292563)\end{array}$} \\
\hline \multirow{2}{*}{$\begin{array}{l}\text { Center } A \\
\text { Numbel }\end{array}$} & Atomic & \multicolumn{2}{|c|}{ Atomic } & \multicolumn{2}{|c|}{ Coordinates (Angstroms } \\
\hline & er Numb & eer & Type & $\mathrm{X}$ & $\mathrm{Y} \quad \mathrm{Z}$ \\
\hline 1 & 13 & 0 & 1.700846 & 1.019319 & 0.891332 \\
\hline 2 & 13 & 0 & 1.100910 & -1.525024 & 1.262685 \\
\hline 3 & 13 & 0 & 0.929495 & 1.620015 & -1.493379 \\
\hline 4 & 13 & 0 & -1.257054 & 0.509795 & -2.155675 \\
\hline 5 & 13 & 0 & 0.447686 & -1.128414 & -1.192642 \\
\hline 6 & 23 & 0 & -0.928561 & 0.366393 & 0.676380 \\
\hline 7 & 6 & 0 & -0.694282 & -0.937357 & 2.217532 \\
\hline 8 & 8 & 0 & -1.754574 & -0.319563 & 2.489647 \\
\hline 9 & 8 & 0 & -0.054712 & -1.892899 & 2.793362 \\
\hline
\end{tabular}

Frequencies -- 68.1067

78.8561

$102.7806 \quad 117.8935$

124.1122 142.3751

$\mathrm{Al}_{5} \mathrm{Cr}(\mathrm{S}=6)$

( $\mathrm{E}=-1297.793348)$

Center Atomic Atomic Coordinates (Angstroms)

Number Number Type $\quad$ X $\quad$ Y $\quad$ Z

$\begin{array}{rrrrrr}1 & 13 & 0 & 0.354385 & 1.309406 & -1.559497 \\ 2 & 13 & 0 & 0.295262 & 2.302010 & 0.843909 \\ 3 & 13 & 0 & 1.485895 & -0.000230 & 0.692789 \\ 4 & 13 & 0 & 0.354235 & -1.308733 & -1.560058\end{array}$




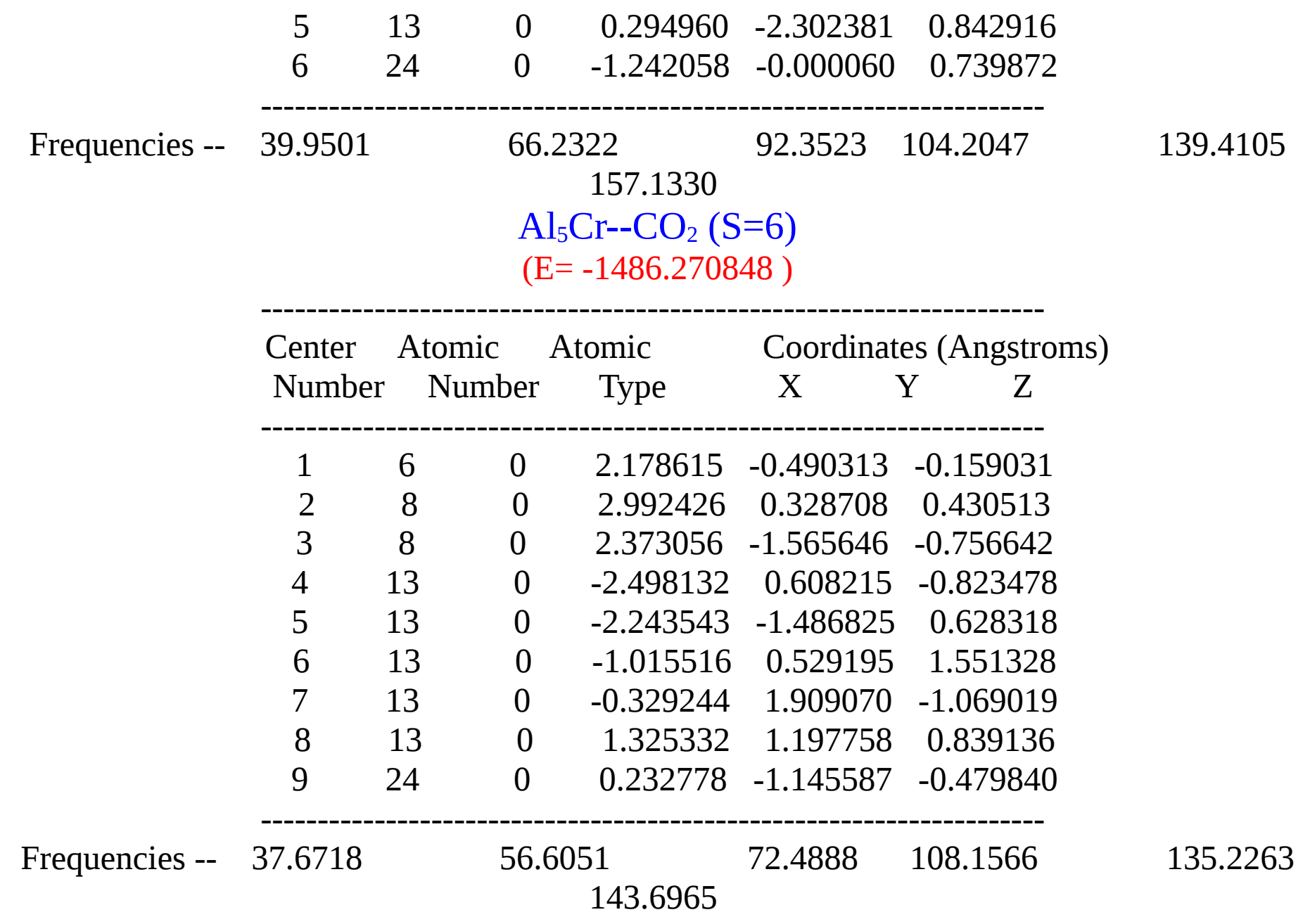

139.4105

$$
\begin{gathered}
\mathrm{Al}_{5} \mathrm{Cr}--\mathrm{CO}_{2}(\mathrm{~S}=6) \\
(\mathrm{E}=-1486.270848)
\end{gathered}
$$

$\mathrm{Al}_{5} \mathrm{Mn}(\mathrm{S}=7)$

(E=-1315.398497)

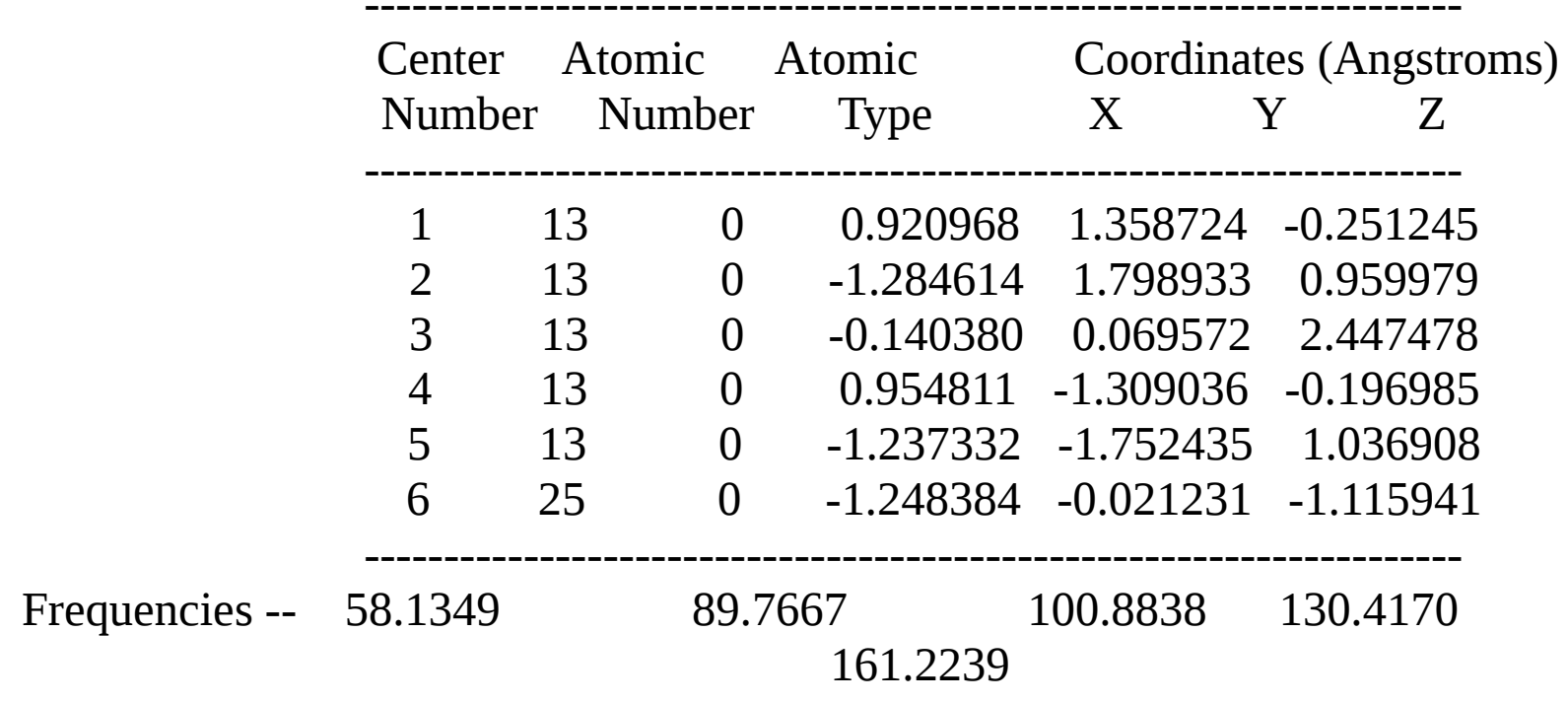

148.7597 


\begin{tabular}{|c|c|c|c|c|c|}
\hline & & & $\begin{array}{l}\mathrm{Al}_{5} \mathrm{Mn}--\mathrm{CO}_{2}( \\
(\mathrm{E}=-1503.8754\end{array}$ & $\begin{array}{l}=7) \\
1 \text { ) }\end{array}$ & \\
\hline & Center & Atomic & Atomic & Coordinat & tes (Angstroms) \\
\hline & Number & Numbe & er Type & $\mathrm{X}$ & $\mathrm{Y} \quad \mathrm{Z}$ \\
\hline & 1 & 13 & 0.958460 & 0.848536 & 0.842526 \\
\hline & 2 & 13 & -0.938876 & 2.631761 & 0.776675 \\
\hline & 3 & 13 & -1.342935 & 0.531295 & 2.293286 \\
\hline & 4 & 13 & 1.331006 & -1.432733 & -0.267919 \\
\hline & 5 & 13 & -0.337863 & -1.754207 & 1.734413 \\
\hline & 6 & 25 & -1.261139 & 0.085117 & -0.584623 \\
\hline & 7 & 6 & -0.218874 & -1.395976 & -1.705847 \\
\hline & 8 & 8 & -1.259213 & -1.152407 & -2.362542 \\
\hline & 9 & 8 & 0.795236 & -2.148383 & -1.987992 \\
\hline Frequencies -- & 50.9037 & & $\begin{array}{l}1.2955 \\
126.1119\end{array}$ & 75.6222 & 89.6227 \\
\hline
\end{tabular}

101.6216

\section{$\mathrm{Al}_{5} \mathrm{Fe}(\mathrm{S}=4)$ \\ $(\mathrm{E}=-1334.933412$ )}

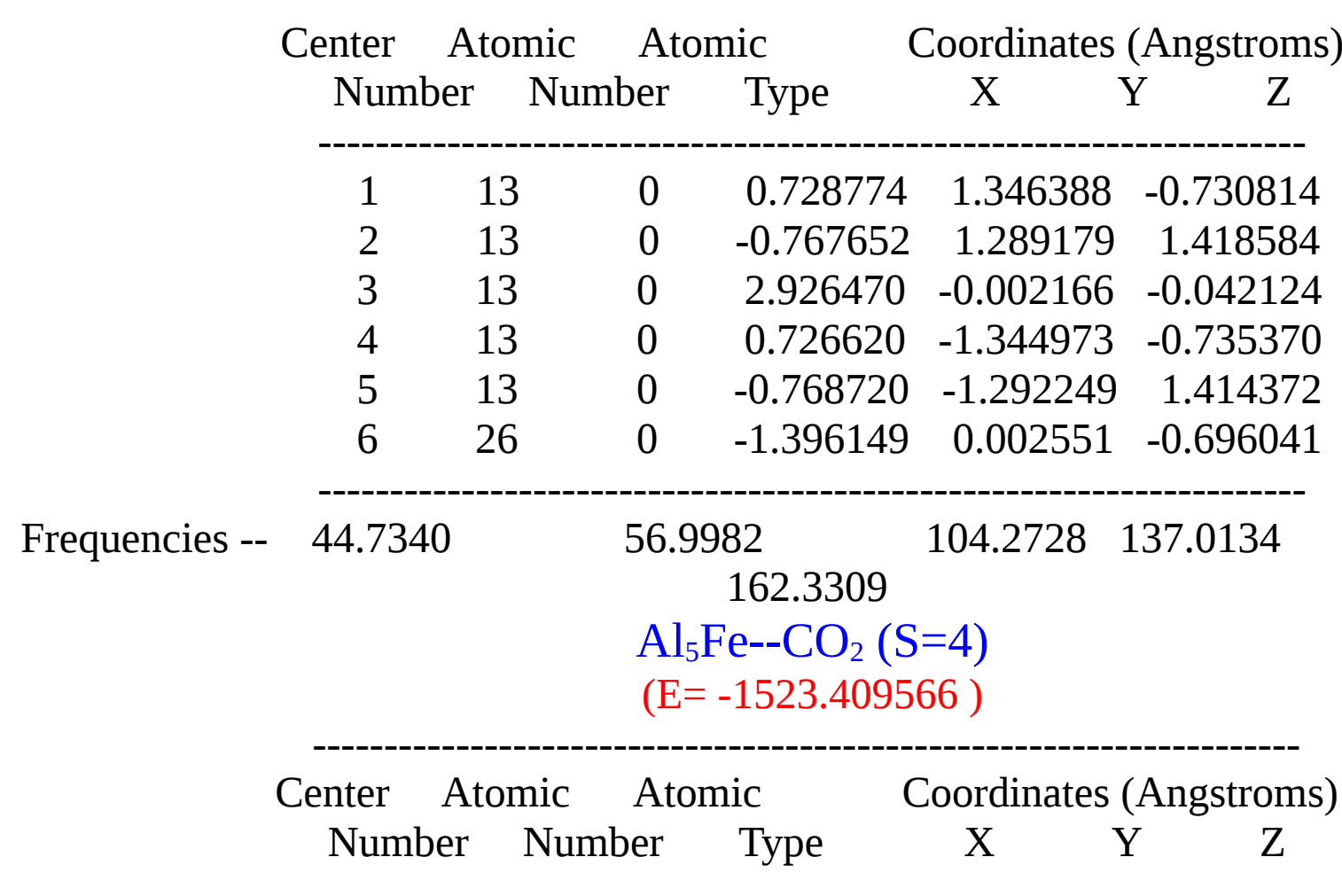




\begin{tabular}{rrrrrr}
1 & 6 & 0 & -1.990154 & -0.853917 & -0.192089 \\
2 & 8 & 0 & -2.951986 & -0.269532 & 0.472866 \\
3 & 8 & 0 & -2.013250 & -1.795055 & -0.994724 \\
4 & 13 & 0 & -0.039180 & 1.514021 & -0.950175 \\
5 & 13 & 0 & -1.550553 & 0.862041 & 1.072175 \\
6 & 13 & 0 & 2.440435 & 1.048264 & -1.113532 \\
7 & 13 & 0 & 2.516592 & -0.851865 & 0.512527 \\
8 & 13 & 0 & 0.842444 & 0.530567 & 1.755214 \\
9 & 26 & 0 & 0.016545 & -0.945873 & -0.262519 \\
\hline
\end{tabular}

Frequencies -- 43.6643

60.6054

$67.5146 \quad 100.2694$

120.9239

\subsection{7}

$\mathrm{Al}_{5} \mathrm{Co}(\mathrm{S}=3)$

$(\mathrm{E}=-1356.599939)$

Center Atomic Atomic Coordinates (Angstroms)

$\begin{array}{lllll}\text { Number Number Type } & X & Y & Z\end{array}$

$\begin{array}{rrrrrr}1 & 13 & 0 & 1.384902 & -1.532180 & 0.012379 \\ 2 & 13 & 0 & 2.151842 & 1.072698 & 0.076414 \\ 3 & 13 & 0 & 0.013117 & 0.659987 & 1.589301 \\ 4 & 13 & 0 & -1.385597 & -1.534161 & 0.038749 \\ 5 & 13 & 0 & -2.154680 & 1.069742 & 0.117673 \\ 6 & 27 & 0 & -0.008943 & 0.267839 & -0.735310\end{array}$

Frequencies -- 62.4985

94.5658

123.7976

142.2745

146.0053

175.7793

$\mathrm{Al}_{5} \mathrm{Co}--\mathrm{CO}_{2}(\mathrm{~S}=3)$

$(\mathrm{E}=-1545.068494)$

Center Atomic Atomic Coordinates (Angstroms)

Number Number Type $\quad$ X $\quad$ Y $\quad$ Z

$\begin{array}{rrrrrr}1 & 13 & 0 & 1.515529 & -1.690240 & 0.449881 \\ 2 & 13 & 0 & 2.215546 & 0.799836 & 0.333762 \\ 3 & 13 & 0 & -0.000731 & 0.913673 & 1.535126 \\ 4 & 13 & 0 & -1.086489 & -1.665965 & 0.356264 \\ 5 & 13 & 0 & -2.206368 & 0.727451 & 0.341555\end{array}$




$$
\begin{array}{cccccccc} 
& 6 & 27 & 0 & 0.074905 & 0.111417 & -0.868144 & \\
& 7 & 6 & 0 & -1.542204 & 0.689256 & -1.757910 & \\
8 & 8 & 0 & -1.003077 & 0.616814 & -2.863485 & \\
\text { Frequencies -- } & 59.7401 & 8 & 0 & -2.735408 & 1.123648 & -1.417325 & \\
- \hdashline & 63.2611 & 73.2477 & 86.2902 & 118.4401
\end{array}
$$

\section{$\mathrm{Al}_{5} \mathrm{Ni}(\mathrm{S}=2)$ \\ $(\mathrm{E}=-1380.840832)$}

\begin{tabular}{lcccc} 
Center & Atomic & Atomic & & \multicolumn{2}{c}{ Coordinates (Angstroms) } \\
Number & Number & Type & X & Y C Z
\end{tabular}

$\begin{array}{rrrrrr}1 & 13 & 0 & -2.030476 & 1.065662 & 0.009430 \\ 2 & 13 & 0 & -1.490834 & -1.428873 & 0.273023 \\ 3 & 13 & 0 & 0.000169 & 0.616120 & 1.566677 \\ 4 & 13 & 0 & 2.030783 & 1.065077 & 0.009219 \\ 5 & 13 & 0 & 1.490457 & -1.429310 & 0.272854 \\ 6 & 28 & 0 & -0.000047 & 0.019185 & -0.992105\end{array}$

\begin{tabular}{|c|c|c|c|c|c|}
\hline \multirow{2}{*}{$\begin{array}{l}\text { Center } \\
\text { Number }\end{array}$} & \multicolumn{2}{|c|}{ Atomic } & \multirow{2}{*}{$\begin{array}{l}\text { Atomic } \\
\text { Type }\end{array}$} & \multicolumn{2}{|c|}{ Coordinates (Angstror } \\
\hline & & Number & & $\mathrm{X}$ & $\mathrm{Y} \quad \mathrm{Z}$ \\
\hline 1 & 6 & 0 & -2.129892 & -0.530941 & -0.183962 \\
\hline 2 & 8 & 0 & -2.408042 & -1.542374 & -0.813930 \\
\hline 3 & 8 & 0 & -2.887705 & 0.286560 & 0.508135 \\
\hline 4 & 13 & 0 & 2.017295 & -1.567590 & 0.437714 \\
\hline 5 & 13 & 0 & 2.703918 & 0.621734 & -0.632472 \\
\hline 6 & 13 & 0 & 0.953803 & 0.405309 & 1.655391 \\
\hline 7 & 13 & 0 & -1.321266 & 1.289022 & 0.810525 \\
\hline 8 & 13 & 0 & 0.276282 & 1.579862 & -1.257073 \\
\hline 9 & 28 & 0 & -0.183013 & -0.639536 & -0.341940 \\
\hline
\end{tabular}

Frequencies -- 49.6335

$$
\begin{array}{ccc}
79.7670 & 134.9277 & 150.2618
\end{array}
$$

154.6583

$$
\begin{aligned}
& \mathrm{Al}_{5} \mathrm{Ni}--\mathrm{CO}_{2}(\mathrm{~S}=2) \\
& (\mathrm{E}=-1569.312356)
\end{aligned}
$$


Frequencies -- 58.3809

68.2384

$84.0002 \quad 102.9350$

129.3764

137.0150

\begin{tabular}{|c|c|c|c|c|c|}
\hline & & & $\begin{array}{r}\mathrm{Al}_{5} \mathrm{Cu}(\mathrm{S}=1 \\
(\mathrm{E}=-1407.6625\end{array}$ & & \\
\hline & Center & Atomic & Atomic & Coordinat & tes (Angstroms) \\
\hline & Number & - Number & r Type & $\mathrm{X}$ & $\mathrm{Y} \quad \mathrm{Z}$ \\
\hline & 1 & 13 & 0.934621 & 1.345426 & -0.581814 \\
\hline & 2 & 13 & -0.875758 & 1.313380 & 1.297529 \\
\hline & 3 & 13 & 2.949277 & 0.000123 & 0.392272 \\
\hline & 4 & 13 & 0.934783 & -1.345119 & -0.582216 \\
\hline & 5 & 13 & -0.875596 & -1.313891 & 1.297144 \\
\hline & 6 & 29 & -1.401295 & 0.000026 & -0.808024 \\
\hline Frequencies -- & 51.8574 & & $\begin{array}{l}4.7245 \\
158.5094 \\
\mathrm{Al}_{5} \mathrm{Cu}-\mathrm{CO}_{2}(\mathrm{~S} \\
\mathrm{E}=-1596.1312\end{array}$ & $\begin{aligned} & 77.3521 \\
= & 1) \\
& 6 \text { ) }\end{aligned}$ & 105.5861 \\
\hline
\end{tabular}

Center Atomic Atomic Coordinates (Angstroms)

Number Number Type $\quad X \quad$ Y $\quad$ Z

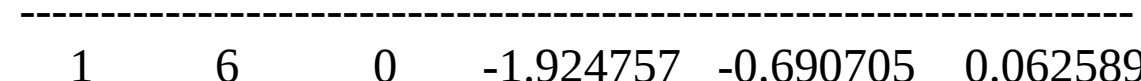

$\begin{array}{llllll}2 & 8 & 0 & -1.711466 & -1.819739 & -0.439772\end{array}$

$\begin{array}{llllll}3 & 8 & 0 & -2.605016 & 0.252166 & -0.627079\end{array}$

$\begin{array}{lllllll}4 & 13 & 0 & 0.945745 & 1.636781 & -0.859999\end{array}$

$\begin{array}{llllll}5 & 13 & 0 & -1.550850 & 1.731405 & -0.259582\end{array}$

$\begin{array}{llllll}6 & 13 & 0 & 2.705771 & -0.197138 & -0.617149\end{array}$

$\begin{array}{llllll}7 & 13 & 0 & 1.629959 & 0.055120 & 1.669362\end{array}$

$\begin{array}{llllll}8 & 13 & 0 & -0.907635 & 0.153855 & 1.753970\end{array}$

$\begin{array}{llllll}9 & 29 & 0 & 0.321442 & -0.968215 & -0.486604\end{array}$

Frequencies -- 53.6898

80.9688

$93.3633 \quad 115.8151$

127.8611 135.5415

146.1780 


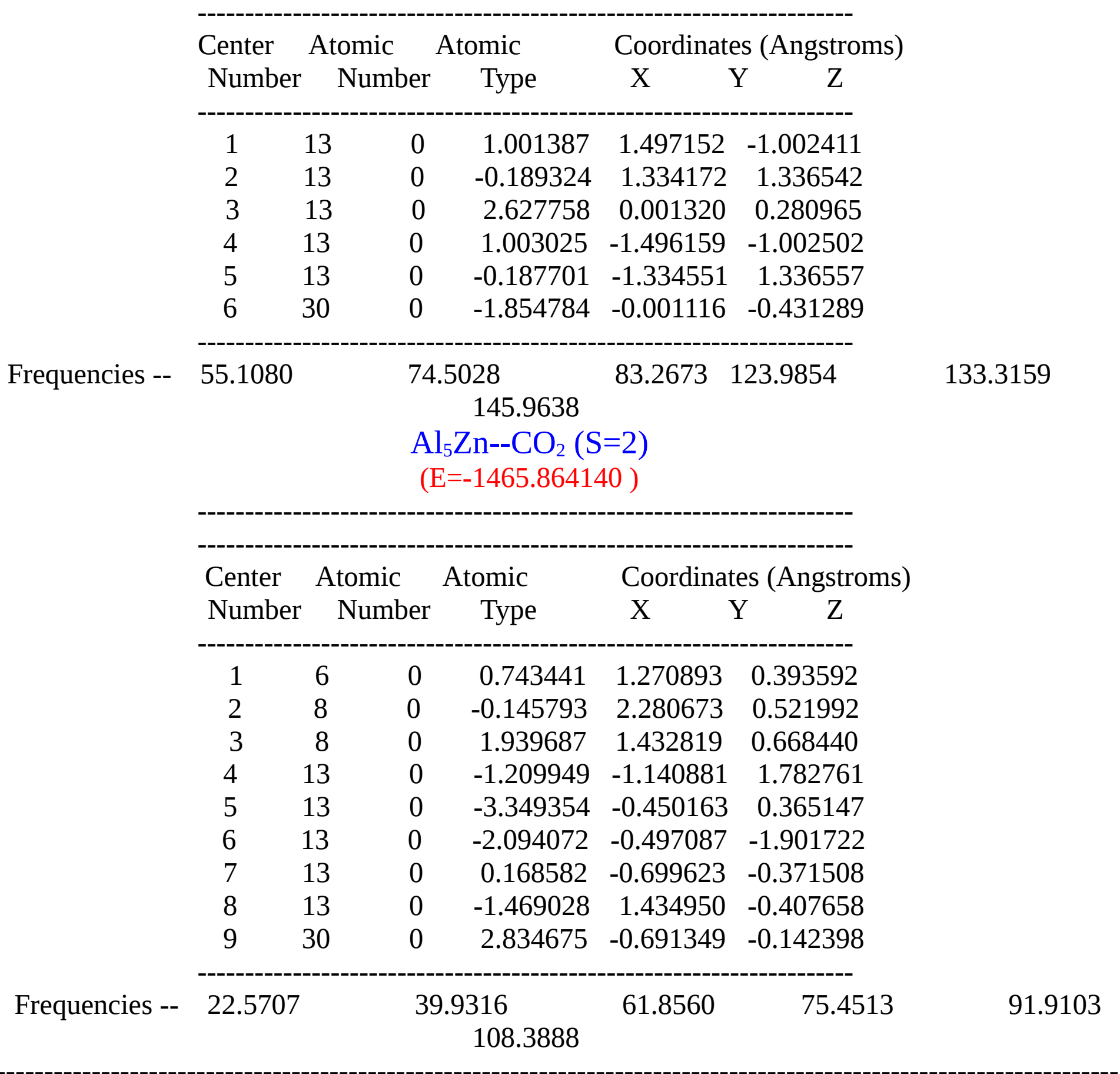

(A)Impure clusters of $\mathrm{Al}_{7} \mathrm{M}(\mathrm{c})$ series

$\mathrm{Al}_{7} \mathrm{Sc}(\mathrm{S}=3)$

$(E=-1742.608369$ )

Center Atomic Atomic

Number Number Type
Coordinates (Angstroms)

$\begin{array}{lll}\mathrm{X} & \mathrm{Y} & \mathrm{Z}\end{array}$ 


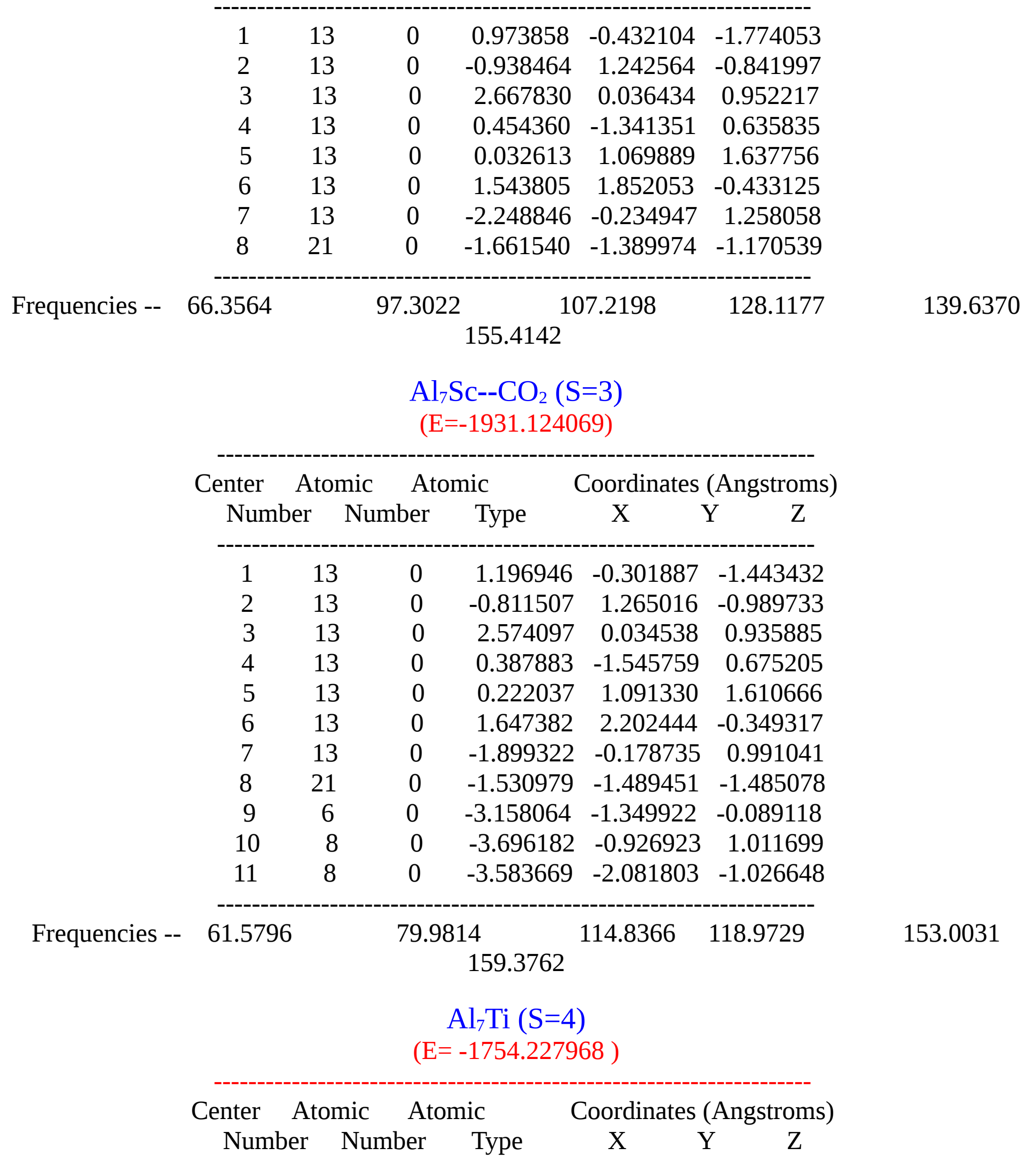




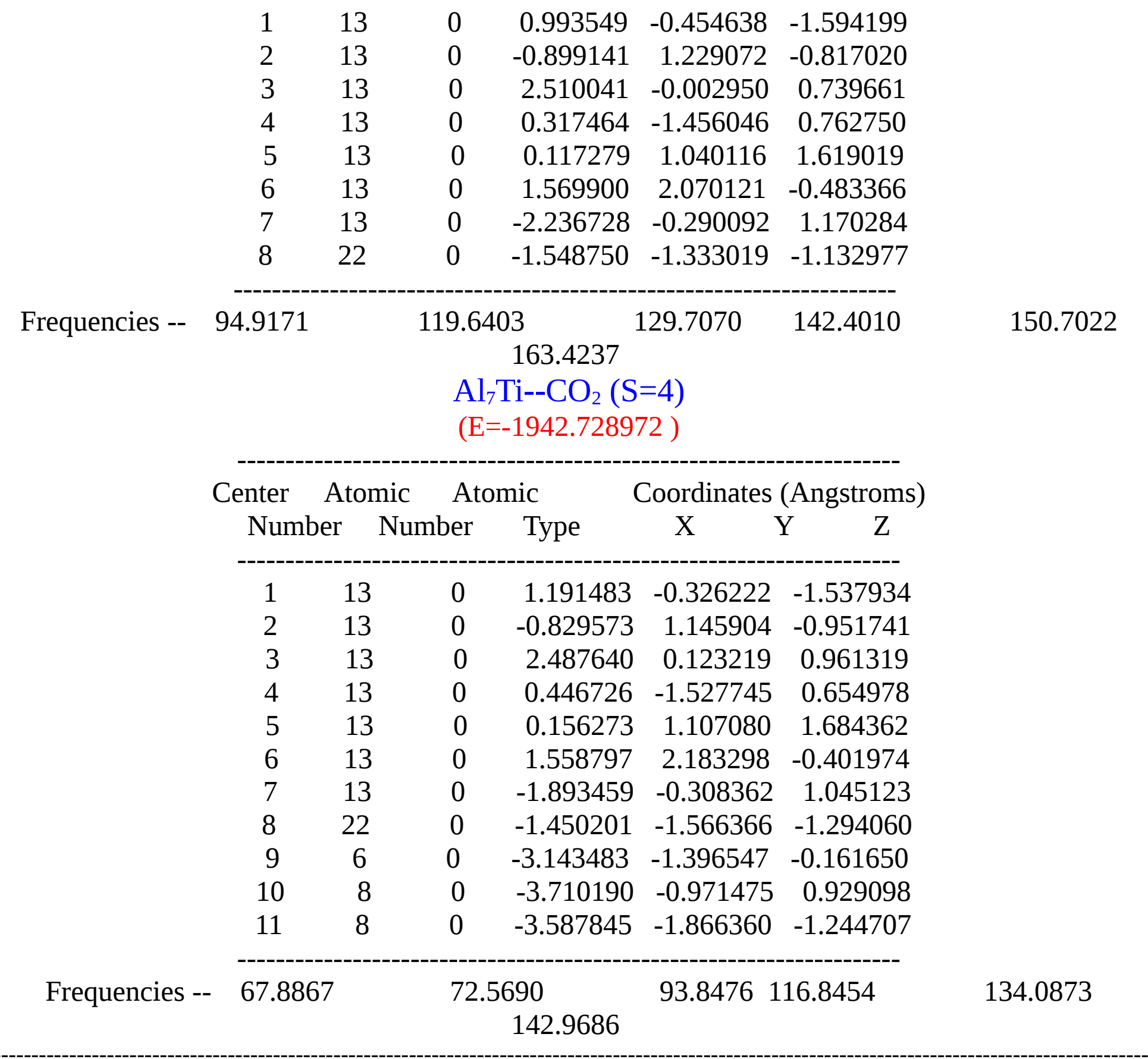

$$
\begin{gathered}
\mathrm{Al}_{7} \mathrm{~V}(\mathrm{~S}=5) \\
(\mathrm{E}=-1767.485271)
\end{gathered}
$$

Center Atomic Atomic Coordinates (Angstroms)

Number Number Type $\quad X \quad$ Y $\quad$ Z

$\begin{array}{llllll}1 & 13 & 0 & 1.196946 & -0.301887 & -1.443432\end{array}$




$\begin{array}{rrrrrr}2 & 13 & 0 & -0.811507 & 1.265016 & -0.989733 \\ 3 & 13 & 0 & 2.574097 & 0.034538 & 0.935885 \\ 4 & 13 & 0 & 0.387883 & -1.545759 & 0.675205 \\ 5 & 13 & 0 & 0.222037 & 1.091330 & 1.610666 \\ 6 & 13 & 0 & 1.647382 & 2.202444 & -0.349317 \\ 7 & 13 & 0 & -1.899322 & -0.178735 & 0.991041 \\ 8 & 21 & 0 & -1.530979 & -1.489451 & -1.485078 \\ 9 & 6 & 0 & -3.158064 & -1.349922 & -0.089118 \\ 10 & 8 & 0 & -3.696182 & -0.926923 & 1.011699 \\ 11 & 8 & 0 & -3.583669 & -2.081803 & -1.026648\end{array}$

Frequencies -- 78.3741

114.8716

$118.3695 \quad 123.1934$

155.5904

$\mathrm{Al}_{7} \mathrm{~V}-\mathrm{CO}_{2}(\mathrm{~S}=5)$

$(\mathrm{E}=-1955.983843)$

Center Atomic Atomic Coordinates (Angstroms)

\begin{tabular}{rrrrrr} 
Number & Number & \multicolumn{1}{c}{ Type } & \multicolumn{1}{c}{ X } & \multicolumn{1}{c}{$Y$} & Z \\
------------------ \\
1 & 13 & 0 & 0.759896 & -1.849152 & 0.014302 \\
2 & 13 & 0 & -0.242385 & -0.149455 & -1.635260 \\
3 & 13 & 0 & 2.452316 & 0.044987 & 1.253093 \\
4 & 13 & 0 & -0.128000 & -0.121202 & 1.699065 \\
5 & 13 & 0 & 1.005231 & 1.881693 & -0.025267 \\
6 & 13 & 0 & 2.361086 & 0.024150 & -1.372554 \\
7 & 13 & 0 & -1.528992 & 1.520031 & 0.063493 \\
8 & 23 & 0 & -2.102296 & -1.044573 & 0.105999 \\
9 & 6 & 0 & -3.354575 & 0.521863 & 0.130666 \\
10 & 8 & 0 & -3.407749 & 1.831278 & 0.121075 \\
11 & 8 & 0 & -4.231772 & -0.365125 & 0.165101
\end{tabular}

Frequencies --

61.1239

74.9992

$90.7702 \quad 106.5906$

139.5182 144.8553

144.2708 


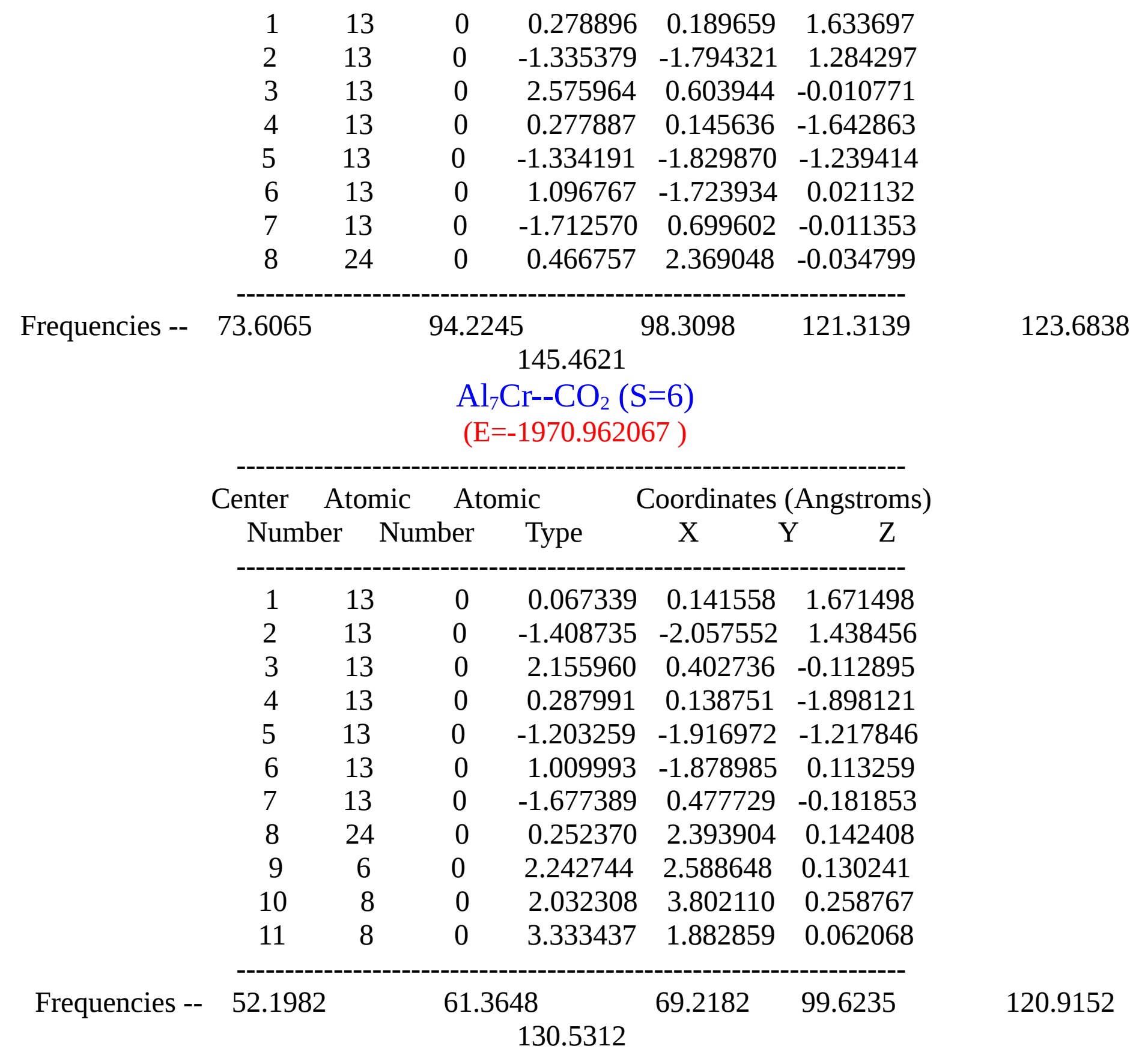

\section{$\mathrm{Al}_{7} \mathrm{Mn}(\mathrm{S}=5)$ \\ $(\mathrm{E}=-1800.093892)$}

Center Atomic Atomic

$\begin{array}{cccccc}\text { Number } & \text { Number } & \text { Type } & X & Y & Z \\ - & 13 & 0 & 0.352943 & 0.283419 & 1.737269 \\ 2 & 13 & 0 & -1.279934 & -1.675745 & 1.208854\end{array}$




$\begin{array}{rrrrrr}3 & 13 & 0 & 2.485304 & 0.618345 & 0.019150 \\ 4 & 13 & 0 & 0.227365 & 0.126394 & -1.670759 \\ 5 & 13 & 0 & -1.386402 & -1.970065 & -1.372716 \\ 6 & 13 & 0 & 1.089061 & -1.664372 & 0.035159 \\ 7 & 13 & 0 & -1.656238 & 0.707820 & 0.022127 \\ 8 & 25 & 0 & 0.482033 & 2.233968 & 0.020840\end{array}$

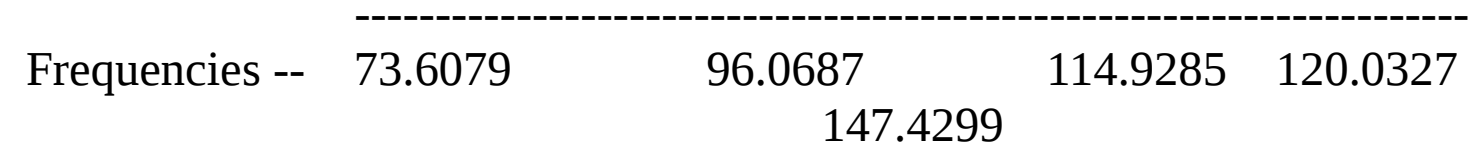

137.9713

$$
\begin{gathered}
\mathrm{Al}_{7} \mathrm{Mn}--\mathrm{CO}_{2}(\mathrm{~S}=5) \\
(\mathrm{E}=-1988.574567)
\end{gathered}
$$

Center Atomic Atomic Coordinates (Angstroms)

\begin{tabular}{rrrrrr} 
Number & Number & \multicolumn{1}{c}{ Type } & X & $Y$ & $Z$ \\
----------------- \\
1 & 13 & 0 & 0.212407 & 0.249378 & 1.753517 \\
2 & 13 & 0 & -1.325695 & -1.758133 & 1.165177 \\
3 & 13 & 0 & 2.201468 & 0.498750 & -0.016685 \\
4 & 13 & 0 & 0.088191 & 0.092347 & -1.644809 \\
5 & 13 & 0 & -1.241847 & -2.199395 & -1.405262 \\
6 & 13 & 0 & 1.109271 & -1.836920 & 0.100094 \\
7 & 13 & 0 & -1.787253 & 0.597148 & 0.030069 \\
8 & 25 & 0 & 0.249592 & 2.281326 & 0.111789 \\
9 & 6 & 0 & 2.316346 & 2.582113 & 0.042837 \\
10 & 8 & 0 & 2.120242 & 3.803153 & 0.076502 \\
11 & 8 & 0 & 3.446715 & 1.901226 & -0.081053
\end{tabular}

Frequencies -- 58.9538

$62.1554 \quad 90.3975 \quad 108.6793$

130.0657 141.7148

\section{$\mathrm{Al}_{7} \mathrm{Fe}(\mathrm{S}=4)$ \\ $(\mathrm{E}=-1819.621757)$}

Center Atomic Atomic Coordinates (Angstroms)

\begin{tabular}{rrrrrr} 
Number & Number & Type & X & Y & Z \\
\hline 1 & 13 & 0 & 1.135119 & -0.017686 & 1.552192 \\
2 & 13 & 0 & -0.883326 & -1.401608 & 0.602710 \\
3 & 13 & 0 & 2.408732 & 0.063054 & -1.025887
\end{tabular}




$\begin{array}{rrrrrr}4 & 13 & 0 & 0.381781 & 1.586758 & -0.386848 \\ 5 & 13 & 0 & -0.106387 & -0.626042 & -1.873774 \\ 6 & 13 & 0 & 1.442482 & -2.220918 & -0.267420 \\ 7 & 13 & 0 & -2.186553 & 0.605954 & -0.812043 \\ 8 & 26 & 0 & -1.161031 & 0.922994 & 1.452025\end{array}$

Frequencies -- 83.1135

$$
\begin{gathered}
122.1503 \quad 131.2198 \\
152.0174 \\
\mathrm{Al}_{7} \mathrm{Fe}--\mathrm{CO}_{2}(\mathrm{~S}=4) \\
(\mathrm{E}=-2008.100325)
\end{gathered}
$$$$
134.0498
$$

146.5922

Center Atomic Atomic Coordinates (Angstroms)

Number Number Type $\quad$ X $\quad$ Y $\quad$ Z

$\begin{array}{rrrrrr}1 & 13 & 0 & 1.243895 & -0.093966 & 1.616266 \\ 2 & 13 & 0 & -0.831753 & -1.420368 & 0.585065 \\ 3 & 13 & 0 & 2.497740 & -0.055543 & -1.440040 \\ 4 & 13 & 0 & 0.628025 & 1.433072 & -0.377377 \\ 5 & 13 & 0 & -0.085670 & -0.808927 & -2.003018 \\ 6 & 13 & 0 & 1.557649 & -2.073613 & -0.187245 \\ 7 & 13 & 0 & -1.895380 & 0.614529 & -0.846192 \\ 8 & 26 & 0 & -1.070348 & 0.912779 & 1.469510 \\ 9 & 6 & 0 & -2.997386 & 1.270523 & 0.941173 \\ 10 & 8 & 0 & -3.597805 & 1.502081 & 1.980835 \\ 11 & 8 & 0 & -3.501139 & 1.320209 & -0.312249\end{array}$

Frequencies -- 53.8896

64.7744

91.2618115 .2368

\begin{tabular}{|c|c|c|c|c|c|}
\hline \multirow{2}{*}{$\begin{array}{l}\text { Center } \\
\text { Number }\end{array}$} & \multicolumn{2}{|c|}{ Atomic } & \multirow{2}{*}{$\begin{array}{l}\text { Atomic } \\
\text { Type }\end{array}$} & \multicolumn{2}{|c|}{ Coordinates (Angstro } \\
\hline & & Number & & $\mathrm{X}$ & $\mathrm{Y} \quad \mathrm{Z}$ \\
\hline 1 & 13 & 0 & -0.264958 & 0.066202 & -1.687919 \\
\hline 2 & 13 & 0 & 0.883378 & 1.831440 & -0.000374 \\
\hline 3 & 13 & 0 & 2.319288 & -0.068910 & -1.272332 \\
\hline 4 & 13 & 0 & 0.455079 & -1.835429 & -0.005917 \\
\hline 5 & 13 & 0 & -0.234713 & 0.064219 & 1.687615 \\
\hline
\end{tabular}

122.0893 132.9924

\section{$\mathrm{Al}_{7} \mathrm{Co}(\mathrm{S}=3)$ \\ $(E=-1841.280664)$}




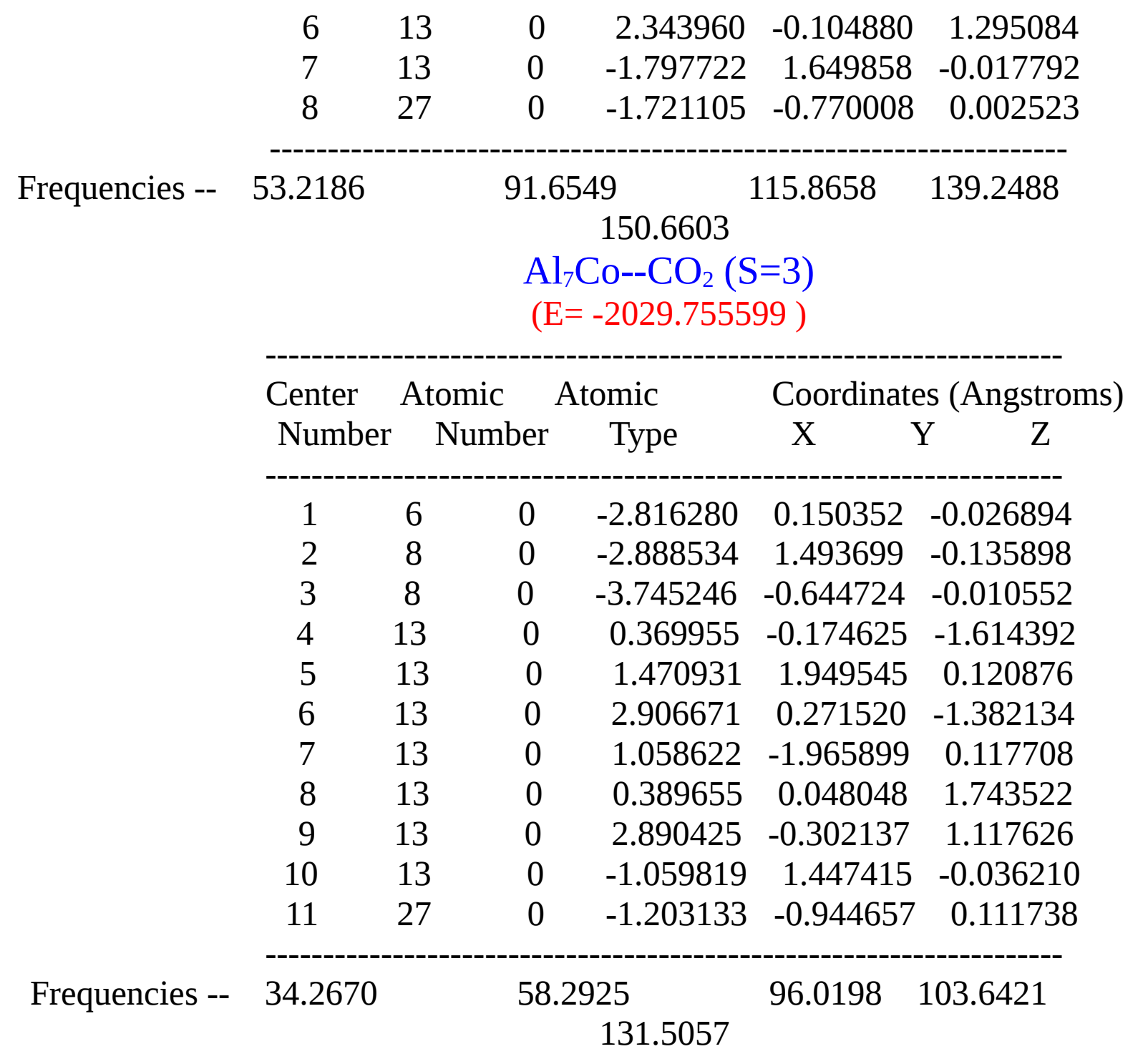

143.5035

$$
\begin{gathered}
\mathrm{Al}_{7} \mathrm{Co}--\mathrm{CO}_{2}(\mathrm{~S}=3) \\
(\mathrm{E}=-2029.755599)
\end{gathered}
$$

Center Atomic Atomic Coordinates (Angstroms)

131.5057

110.7149

$\mathrm{Al}_{7} \mathrm{Ni}(\mathrm{S}=2)$

\begin{tabular}{|c|c|c|c|c|}
\hline \multirow{2}{*}{$\begin{array}{l}\text { Center } \\
\text { Number }\end{array}$} & Atomic & \multirow{2}{*}{$\begin{array}{l}\text { Atomic } \\
\text { Type }\end{array}$} & \multicolumn{2}{|c|}{ Coordinates (Angstrom } \\
\hline & Numbe & & $\begin{array}{ll}X & Y\end{array}$ & $\mathrm{Y} \quad \mathrm{Z}$ \\
\hline 1 & 13 & 0.277002 & 0.056676 & 1.717104 \\
\hline 2 & 13 & -1.032309 & 1.802187 & -0.000194 \\
\hline 3 & 13 & -2.244400 & -0.190334 & 1.298214 \\
\hline 4 & 13 & -0.359687 & -1.852138 & 0.000193 \\
\hline 5 & 13 & 0.276974 & 0.056331 & -1.717108 \\
\hline 6 & 13 & -2.244421 & -0.190642 & -1.298151 \\
\hline 7 & 13 & 1.605626 & 1.752081 & -0.000187 \\
\hline
\end{tabular}

$(\mathrm{E}=-1865.539225)$ 


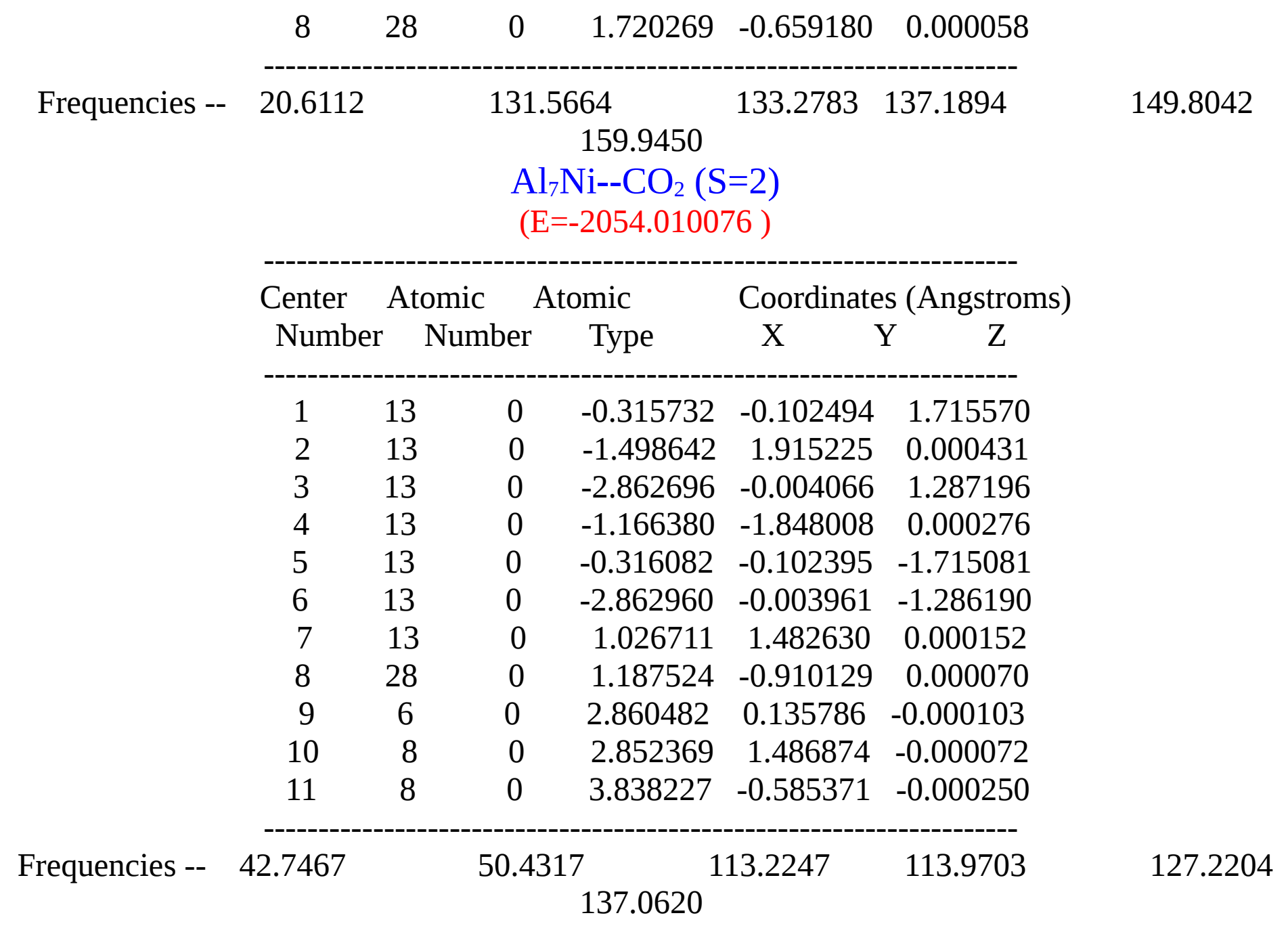

\section{$\mathrm{Al}_{7} \mathrm{Cu}(\mathrm{S}=1)$ \\ $(E=-1892.353158)$}

\begin{tabular}{|c|c|c|c|c|c|}
\hline \multirow{2}{*}{$\begin{array}{l}\text { Center } \\
\text { Number }\end{array}$} & \multicolumn{2}{|c|}{ Atomic } & \multirow{2}{*}{$\begin{array}{l}\text { Atomic } \\
\text { Type }\end{array}$} & \multicolumn{2}{|c|}{ Coordinates (Angstroms) } \\
\hline & & Number & & $\mathrm{X}$ & $\mathrm{Y} \quad \mathrm{Z}$ \\
\hline 1 & 13 & 0 & -0.157661 & 0.175408 & -1.666577 \\
\hline 2 & 13 & 0 & 1.043098 & 1.788313 & 0.000193 \\
\hline 3 & 13 & 0 & 2.339281 & -0.263592 & -1.263765 \\
\hline 4 & 13 & 0 & 0.429843 & -1.860381 & -0.000189 \\
\hline 5 & 13 & 0 & -0.157658 & 0.175046 & 1.666630 \\
\hline 6 & 13 & 0 & 2.339285 & -0.263842 & 1.263711 \\
\hline 7 & 13 & 0 & -1.800940 & 1.927408 & 0.000224 \\
\hline 8 & 29 & 0 & -1.795185 & -0.754896 & -0.000074 \\
\hline
\end{tabular}




\begin{tabular}{|c|c|c|c|c|c|c|c|}
\hline \multirow[t]{14}{*}{ Frequencies -- } & \multicolumn{6}{|c|}{$\begin{array}{c}72.1779 \quad 86.0677 \\
128.8515 \\
\mathrm{Al}_{7} \mathrm{Cu}--\mathrm{CO}_{2}(\mathrm{~S}=1) \\
(\mathrm{E}=-2080.818665)\end{array}$} & \multirow[t]{3}{*}{127.0364} \\
\hline & \multirow{2}{*}{$\begin{array}{l}\text { Center } \\
\text { Number }\end{array}$} & Atomic & \multirow{2}{*}{\multicolumn{2}{|c|}{\begin{tabular}{c}
\multicolumn{2}{c}{ Atomic } \\
er $\quad$ Type
\end{tabular}}} & \multicolumn{2}{|c|}{ Coordinates (Angstroms) } & \\
\hline & & r Numbe & & & $\mathrm{X}$ & $\mathrm{Y} \quad \mathrm{Z}$ & \\
\hline & 1 & 13 & 0 & 0.027346 & -0.018057 & -1.693228 & \\
\hline & 2 & 13 & 0 & 1.148246 & 1.926563 & -0.000290 & \\
\hline & 3 & 13 & 0 & 2.558036 & -0.061544 & -1.241172 & \\
\hline & 4 & 13 & 0 & 0.693335 & -1.833243 & 0.012076 & \\
\hline & 5 & 13 & 0 & -0.007240 & 0.004267 & 1.683102 & \\
\hline & 6 & 13 & 0 & 2.530680 & -0.063695 & 1.277744 & \\
\hline & 7 & 13 & 0 & -1.414993 & 1.572949 & -0.029433 & \\
\hline & 8 & 29 & 0 & -1.781435 & -0.860357 & -0.015879 & \\
\hline & 9 & 6 & & -3.440319 & 0.409610 & -0.037419 & \\
\hline & 10 & 8 & 0 & -3.179867 & 1.793888 & -0.048362 & \\
\hline & 11 & 8 & 0 & -4.577301 & -0.001225 & -0.042256 & \\
\hline Frequencies -- & 25.2425 & & & $\begin{array}{l}.1060 \\
119.2026\end{array}$ & 89.2961 & 91.2374 & 103.8831 \\
\hline
\end{tabular}

\section{$\mathrm{Al}_{7} \mathrm{Zn}(\mathrm{S}=2)$ \\ $(E=-1762.075985)$}

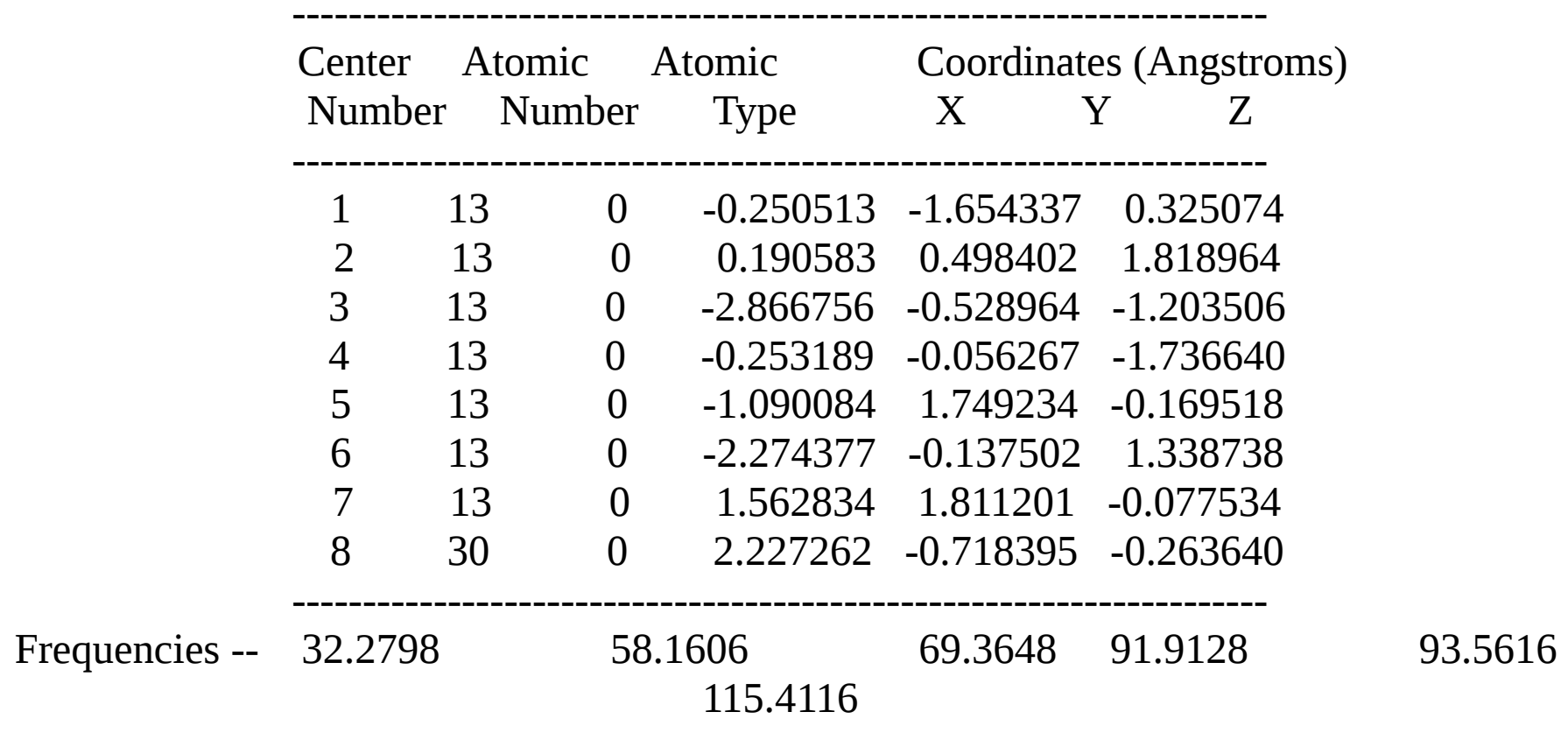




\begin{tabular}{|c|c|c|c|c|c|c|}
\hline & & & & $\begin{array}{l}\mathrm{l}_{7} \mathrm{Zn}--\mathrm{CO}_{2} \\
\mathrm{E}=-1950.5413\end{array}$ & $\begin{array}{l}=2) \\
95)\end{array}$ & \\
\hline & Center & Atc & omic & Atomic & Coordinate & tes (Angstroms) \\
\hline & Number & & Number & Type & $\mathrm{X}$ & $\mathrm{Y} \quad \mathrm{Z}$ \\
\hline & 1 & 6 & 0 & 0.970528 & -1.759685 & 0.207916 \\
\hline & 2 & 8 & 0 & 0.319364 & -2.867250 & -0.097837 \\
\hline & 3 & 8 & 0 & 2.212518 & -1.659719 & 0.083388 \\
\hline & 4 & 13 & 0 & -1.266699 & -1.899032 & -0.325662 \\
\hline & 5 & 13 & 0 & -0.038911 & -0.077676 & 1.167796 \\
\hline & 6 & 13 & 0 & -3.011541 & -0.045444 & -1.015102 \\
\hline & 7 & 13 & 0 & -0.568183 & 0.439523 & -1.589694 \\
\hline & 8 & 13 & 0 & -1.790801 & 2.091616 & 0.422389 \\
\hline & 9 & 13 & 0 & -2.562870 & -0.204597 & 1.601214 \\
\hline & 10 & 13 & 0 & 0.773047 & 2.209001 & -0.057758 \\
\hline & 11 & 30 & 0 & 2.772158 & 0.455209 & -0.375669 \\
\hline Frequencies -- & 34.7387 & & & $\begin{array}{l}.7103 \\
124.3730\end{array}$ & 69.8219 & 96.7331 \\
\hline
\end{tabular}

115.3905 
Table S23

Comparison between the data of standard DFT and DFT-D results for PBE-PBE functional

\begin{tabular}{|c|c|c|c|c|c|c|c|c|c|c|}
\hline & & & & Functional & PBE-PBE & & & & & \\
\hline & & & & & & & & & & \\
\hline & & H & & & Al5M & & & G & & \\
\hline & DFT & DFT-D & difference & Avg & & & DFT & DFT-D & difference & Avg \\
\hline Sc & -42.0638778 & -45.9845603 & 3.92068248 & 3.2820028 & & Sc & -31.4144056 & -35.1982909 & 3.7838853 & 3.24454045 \\
\hline $\mathrm{Ti}$ & -40.2779844 & -43.9024821 & 3.62449776 & & & $\mathrm{Ti}$ & -28.2228898 & -31.8178946 & 3.59500479 & \\
\hline V & -30.9531858 & -34.9504245 & 3.9972387 & & & V & -19.0568512 & -22.6989192 & 3.64206804 & \\
\hline $\mathrm{Cr}$ & -20.0138039 & -24.0066501 & 3.99284613 & & & $\mathrm{Cr}$ & -9.12148536 & -12.7033124 & 3.58182708 & \\
\hline $\mathrm{Mn}$ & -21.9440247 & -23.3791401 & 1.43511537 & & & $\mathrm{Mn}$ & -11.2274089 & -13.0101648 & 1.78275591 & \\
\hline $\mathrm{Fe}$ & -19.9115198 & -23.1030357 & 3.19151586 & & & $\mathrm{Fe}$ & -8.72740908 & -12.017444 & 3.29003493 & \\
\hline Co & -14.5023836 & -18.1996725 & 3.69728892 & & & Co & -4.31852382 & -7.81814709 & 3.49962327 & \\
\hline $\mathrm{Ni}$ & -15.7774839 & -20.0947527 & 4.3172688 & & & $\mathrm{Ni}$ & -5.48694744 & -9.31601346 & 3.82906602 & \\
\hline $\mathrm{Cu}$ & -14.3028354 & -18.5975139 & 4.29467844 & & & $\mathrm{Cu}$ & -2.79555705 & -6.55245942 & 3.75690237 & \\
\hline \multirow[t]{5}{*}{$\mathrm{Zn}$} & -11.7934229 & -12.1423185 & 0.34889556 & & & $Z n$ & -0.67959333 & -2.36383017 & 1.68423684 & \\
\hline & & & & & & & & & & \\
\hline & & & & & Al7M(c) & & & & & \\
\hline & & H & & & & & & G & & \\
\hline & DFT & DFT-D & difference & Avg & & & DFT & DFT-D & difference & Avg \\
\hline Sc & -45.3652079 & -48.1099367 & 2.74472874 & 3.19484166 & & Sc & -33.4764035 & -36.2638029 & 2.78739942 & 3.12550181 \\
\hline $\mathrm{Ti}$ & -35.3545409 & -38.6809714 & 3.32643051 & & & $\mathrm{Ti}$ & -24.3800185 & -27.738452 & 3.35843352 & \\
\hline V & -34.012297 & -37.2577787 & 3.24548172 & & & V & -22.6606411 & -25.9726389 & 3.31199778 & \\
\hline $\mathrm{Cr}$ & -22.1052948 & -25.758658 & 3.65336322 & & & $\mathrm{Cr}$ & -11.0485686 & -14.7979408 & 3.74937225 & \\
\hline $\mathrm{Mn}$ & -22.6757014 & -25.821409 & 3.14570763 & & & $\mathrm{Mn}$ & -12.0023838 & -15.0232169 & 3.02083314 & \\
\hline $\mathrm{Fe}$ & -21.3064745 & -24.4578298 & 3.15135522 & & & $\mathrm{Fe}$ & -9.79668612 & -13.96147 & 4.16478387 & \\
\hline Co & -18.3364697 & -22.2816251 & 3.94515537 & & & Co & -8.94452754 & -11.575677 & 2.63114943 & \\
\hline $\mathrm{Ni}$ & -16.567519 & -19.6278853 & 3.06036627 & & & $\mathrm{Ni}$ & -5.89482894 & -8.66465808 & 2.76982914 & \\
\hline $\mathrm{Cu}$ & -13.4456568 & -16.2531365 & 2.80747974 & & & $\mathrm{Cu}$ & -3.08044659 & -5.90737914 & 2.82693255 & \\
\hline
\end{tabular}

